Eduardo Tadashi Katsuno

\title{
Topology Optimization of the Superficial Painting Pattern on Model-Scale Propellers applied for Hydrodynamic Performance
}

São Paulo, Brazil 

Eduardo Tadashi Katsuno

\title{
Topology Optimization of the Superficial Painting Pattern on Model-Scale Propellers applied for Hydrodynamic Performance
}

\author{
Revised version
}

Master's Dissertation submitted to the Escola Politécnica of the University of São Paulo in partial fulfillment of the requirements for the degree of Master of Science

Field of Study:

Control and Mechanical Automation

Engineering (3152)

Supervisor:

Prof. Dr. Emílio Carlos Nelli Silva

Co-supervisor:

Dr. João Lucas Dozzi Dantas

São Paulo, Brazil 
Autorizo a reprodução e divulgação total ou parcial deste trabalho, por qualquer meio convencional ou eletrônico, para fins de estudo e pesquisa, desde que citada a fonte.

Este exemplar foi revisado e corrigido em relação à versão original, sob responsabilidade única do autor e com a anuência de seu orientador.

São Paulo, de de

Assinatura do autor:

Assinatura do orientador:

\section{Catalogação-na-publicação}

\section{Katsuno, Eduardo Tadashi}

Topology Optimization of the Superficial Painting Pattern on Model-Scale Propellers applied for Hydrodynamic Performance / E. T. Katsuno -- versão corr. -- São Paulo, 2019.

$158 \mathrm{p}$.

Dissertação (Mestrado) - Escola Politécnica da Universidade de São Paulo. Departamento de Engenharia Mecatrônica e de Sistemas Mecânicos.

1.Topology Optimization 2.CFD 3.Propeller 4.Adjoint Method 5.SHS I.Universidade de São Paulo. Escola Politécnica. Departamento de Engenharia Mecatrônica e de Sistemas Mecânicos II.t. 


\section{Acknowledgements}

Agradeço à minha família, pelo apoio e por sempre acreditar e investir em minha educação.

To João Lucas Dozzi Dantas (Doctor Dantas), for having presented this theme and for CFD teaching and discussions since I was an intern. Also, thanks for your support, guidance, and for being able to get me to attend multiple conferences.

To prof. Emílio, for his guidance, charging, and discussions throughout this dissertation. Furthermore, for always being open-door and having promptly welcomed me, trusted in the theme and accepted me to be my supervisor during my undergraduate and in this master's.

To Felipe Santos de Castro (Mr. De Castro), for friendship, companionship, some random discussions, and for correcting this dissertation, which I really appreciated.

To my aerodesign friends, especially (in alphabetic order): Brunex, André C., Erva, Joãaao, Liba, México, Morth and Rods, for their friendship.

To my friends Rodolfo (paganoispeloamordedeus) and Coragem, for their companionship, several discussions about the future, and academic life throughout the Master's.

To my laboratory colleagues, André, Ana Gilda, Felipe, Fillipenses, 3G, João, Lucas, and Rodolfo for the good times.

To the Institute for Technological Research (IPT) and Fundação de Apoio ao Instituto de Pesquisas Tecnológicas (FIPT), for providing me the necessary infrastructure for this dissertation; and the Novos Talentos program and my program's advisor, Carlos Daher Padovezi. 



\section{Resumo}

Nas últimas décadas, pesquisas foram realizadas na investigação dos efeitos de pinturas no desempenho de embarcações navais. Parte considerável das pesquisas disponíveis ao público foca em tintas que reduzem a incrustação e o atrito dos cascos dessas embarcações, tais como tintas hidrofóbicas. No entanto, pesquisas aplicadas aos hélices são escassas. Revestir a superfície da pá de um propulsor com tinta com comportamento hidrofóbico altera o arrasto de friç̧ão e consequentemente o torque requerido pelo hélice. Cobrir totalmente uma pá pode afetar negativamente o escoamento em determinadas regiões, reduzindo o desempenho da hélice ou induzindo a cavitação. Este projeto estuda o padrão de distribuição de tinta de aplicação de superfície super-hidrofóbica (SHS) em hélices em escala modelo usando o método de otimização topológica para determinar regiões onde sua aplicação na superfície melhora o desempenho do hélice. As simulações dos hélices foram realizadas com modelos de turbulência, usando as equações de Reynolds-Averaged Navier-Stokes. O método numérico é desenvolvido para modelar o comportamento da camada limite com condições de contorno que impõem o efeito de baixa fricção / hidrofobia para estimar o desempenho de um hélice. Para obter as sensibilidades de otimização topológica, estudase o método adjunto discreto das equações de Navier-Stokes com o modelo hidrofóbico baseado no comprimento do escorregamento. A implementação numérica é feita usando o Star-CCM + como software de fluidodinâmica computacional (CFD), baseado no método dos volumes finitos (MVF), como solver do problema primal e do adjunto, e o otimizador de

ponto-interior (IPOPT). É deduzida a derivação do problema adjunto discreto aplicado ao modelo de super-hidrofobia. A aplicação da otimização topológica aplicado à distribuição hidrofóbica em casos bidimensionais é demonstrada (usando casos de escoamento interno e externo) e para um caso tridimensional (hélice). Apesar de o comportamento do SHS ser simplificado com a adoção do modelo de comprimento de escorregamento, os resultados obtidos mostram que as regiões a serem priorizadas para reduzir a energia dissipada nem sempre são intuitivas. Além disso, dependendo das condições de operação, uma pá totalmente SHS pode não ser a melhor opção. A nova aplicação da otimização topológica de fluidos pode ser estendida para outras aplicações, como problemas de projetos de superfície.

Palavras-chave: Otimização topológica. CFD. Propulsor. Método Adjunto. SHS. 



\section{Abstract}

In the last decades, research was carried out in the investigation of the effects of paintings on the performance of naval vessels. A large proportion of the publicly available research focused on paints that reduce the fouling and friction coefficient of the hulls of these vessels, such as hydrophobic paints. However, research applied to propellers is scarce. Covering the blade surface with paint exhibiting hydrophobic behavior changes the drag of the blades and, consequently, the torque required by the propeller. However, covering a blade fully can adversely affect the flow in certain regions, reducing the performance of the propeller or inducing cavitation inception. This project studies the distribution pattern of the super-hydrophobic surface (SHS) paint on model-scale propellers using the topology optimization method to determine regions where the application of surface treatment leads to improved propeller performance. Propeller simulations were carried out with full turbulent analysis using Reynolds-Averaged Navier-Stokes equations. The numerical method is developed to model the behavior of the boundary layer with boundary conditions that impose the low friction/hydrophobicity effect to predict the performance of a coated propeller. To obtain the topology optimization sensitivities, the discrete adjoint method of the Navier-Stokes equations with the hydrophobic model based on the slip length is studied. The numerical implementation is done by using the Star-CCM+ as the Computational Fluid Dynamics (CFD) software, based on the Finite Volume Method (FVM) as the primal and the adjoint solver, and Interior Point Optimizer (IPOPT) as the optimizer. Derivation of the discrete adjoint problem applied to super-hydrophobic modeling is shown. The application of topology optimization to the hydrophobic distribution on a two-dimensional cases are demonstrated (using internal and external flow as test cases), and for a threedimensional case (propeller). Despite that the SHS behavior is simplified by adopting the slip length model, the obtained results show that regions to be prioritized in order to reduce the dissipated energy is not always intuitive. Furthermore, depending on the operating condition, a fully-SHS case may not be the best option. The novel application of fluid Topology Optimization can be extended for other applications, such as problems of surface design.

Keywords: Topology Optimization. CFD. Propeller. Adjoint method. SHS. 



\section{List of abbreviations and acronyms}

CFD Computational Fluid Dynamics

EEDI Energy Efficiency Design Index

FDM Finite Difference Method

FEM Finite Element Method

FVM Finite Volume Method

IPOPT Interior Point Optimizer

IPT Institute for Technological Research

JNI Java Native Interface

LHS Left-Hand Side

MDAO Multidisciplinary Analysis and Optimization

MRF Moving Reference Frame

PDE Partial Differential Equations

PIV Particle Image Velocimetry

RANS Reynolds-Averaged Navier-Stokes

RBM Rigid Body Motion

RHS Right-Hand Side

s.t. such that

SH Super-Hydrophobic

SHS Super-Hydrophobic Surface

SIMP Simplified Isotropic Material with Penalization

TOM Topology Optimization Method

w.r.t. with respect to 



\section{List of symbols}

$x, X, \alpha \quad$ Scalar variable: lowercase letter, capital letter or greek alphabet.

$\mathbf{x}, \boldsymbol{\alpha} \quad$ Vector variable: lowercase bold letter or bold greek alphabet.

X Tensor variable: capital bold letter.

b Slip length, see Eq. (2.34).

C Cost Function.

$C p \quad$ Pressure coefficient, $C p=\left(p-p_{\infty}\right) / 0.5 \rho v_{\text {ref }}^{2}$.

Cf $\quad$ Friction coefficient, $C f=|\boldsymbol{\tau}| / 0.5 \rho v_{\text {ref }}^{2}$.

c Chord.

cd Drag Coefficient.

$D_{p} \quad$ Propeller diameter.

$\hat{g} \quad$ Surface average of pseudo-density, see Eq. (3.2).

$\hat{g}^{*} \quad$ Surface average limit.

$h \quad$ Characteristic size, see Eq. (6.1).

$J \quad$ Advance ratio, see Eq. (2.1).

$k \quad$ Turbulent kinetic energy, see Eq. (2.26).

$k_{s} \quad$ Scaling factor for optimization problem.

KT Thrust coefficient, see Eq. (2.2).

KQ Torque coefficient, see Eq. (2.3).

m Design variable.

$n \quad$ Propeller rotation, in revolution per second.

p Pressure.

$p_{s} \quad$ Saturation pressure.

$q \quad$ Penalization of Material Model, see Eq. (3.1).

Q Torque.

$R^{2} \quad$ Coefficient of determination.

$R e_{x} \quad$ Chord-based Reynolds Number, see Eq. (2.6). 


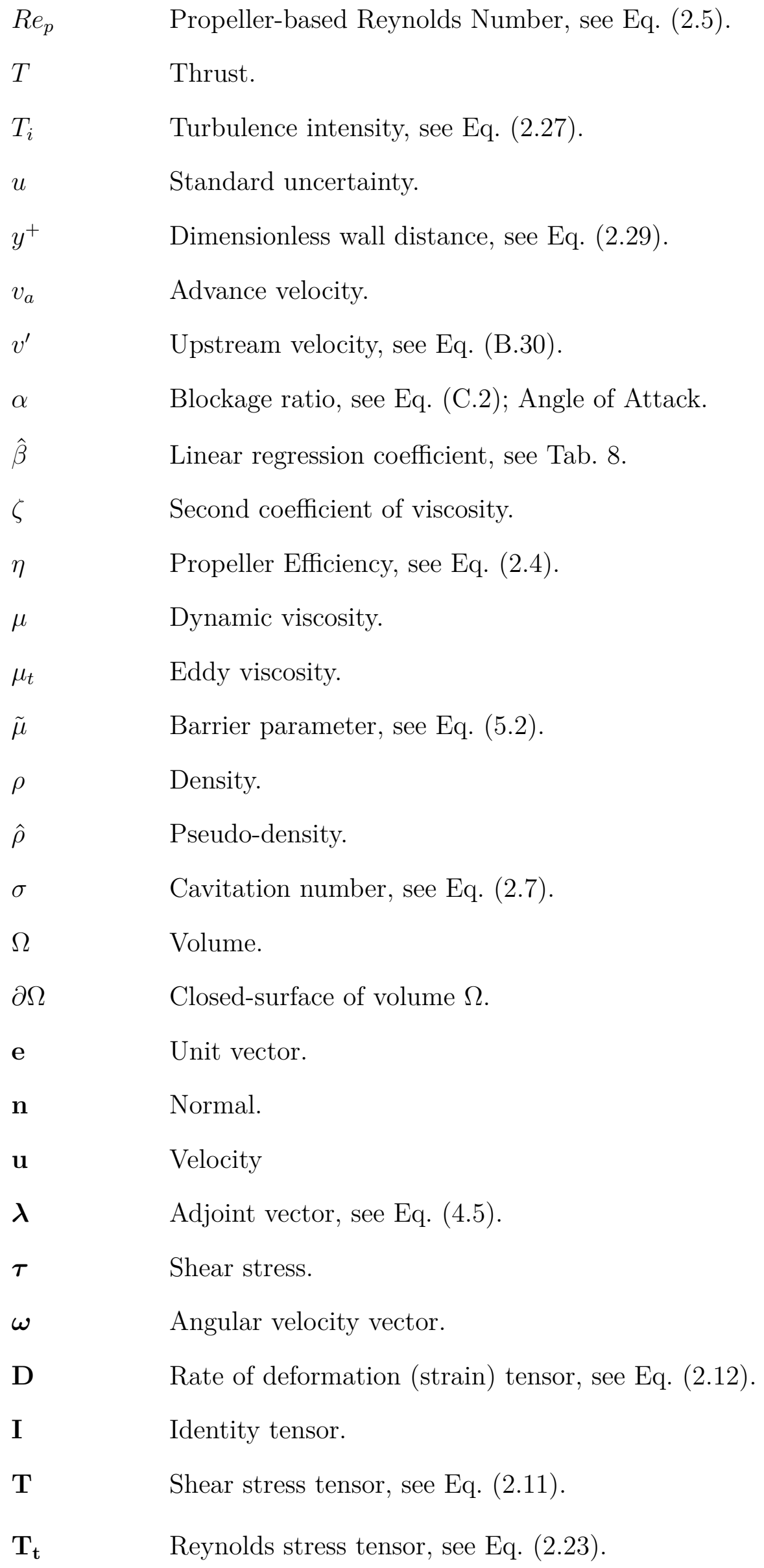




\section{Contents}

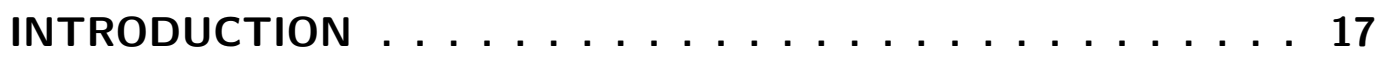

1.1 Super-Hydrophobic Surfaces - SHS . . . . . . . . . . . . . . 18

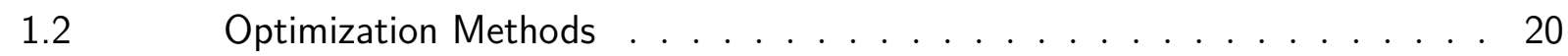

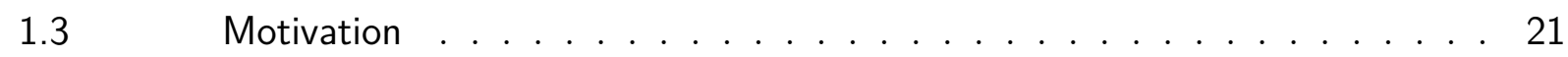

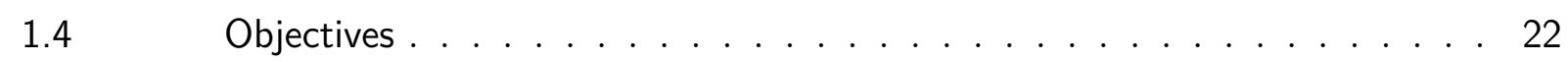

$1.5 \quad$ Document outline . . . . . . . . . . . . . . . . 22

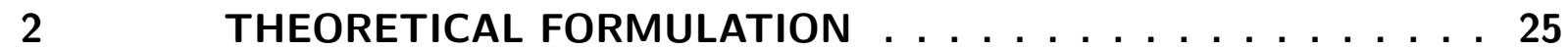

$2.1 \quad$ Basic Concepts of Marine Propellers . . . . . . . . . . . . . . 25

$2.2 \quad$ Fluid Flow Fundamental Equations . . . . . . . . . . . . . 26

$2.3 \quad$ Moving Reference Frame - MRF . . . . . . . . . . . . . . 27

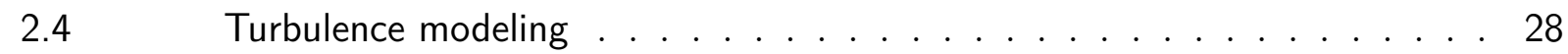

2.5 Boundary Conditions in CFD simulations . . . . . . . . . . 30

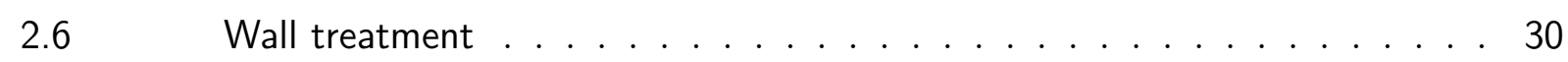

$2.7 \quad$ Cavitation modeling $\ldots \ldots \ldots \ldots \ldots$

2.8 Super-hydrophobic modeling . . . . . . . . . . . . . . . 32

2.9 Finite Volume Method - FVM . . . . . . . . . . . . . . . 33

$2.10 \quad$ Segregated and Coupled Flow Solvers . . . . . . . . . . . . . . 35

3 TOPOLOGY OPTIMIZATION METHOD . . . . . . . . . . . 37

3.1 Topology Optimization applied to Fluid Flow problems . . . . . . . . 37

$3.2 \quad$ Design procedure . . . . . . . . . . . . . . . . . . . 38

$3.3 \quad$ Optimizer . . . . . . . . . . . . . . . . . . . . . 39

$3.4 \quad$ Sensitivities . . . . . . . . . . . . . . . . . . . . . . 39

$3.5 \quad$ Adjoint method . . . . . . . . . . . . . . . . 40

$3.6 \quad$ Material model and Surface Constraint . . . . . . . . . . . . . . 41

$3.7 \quad$ Optimization Formulation $\ldots \ldots \ldots \ldots$. . . . . . . . . 42

4 DISCRETE ADJOINT METHOD FOR NAVIER-STOKES EQUA-

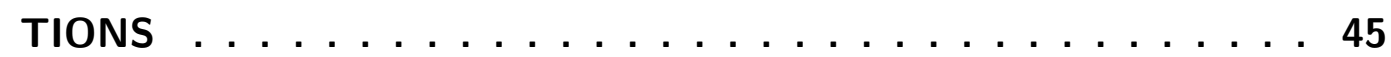

$4.1 \quad$ Adjoint method . . . . . . . . . . . . . . . . . 45

4.2 Derivative of residual with respect to slip length . . . . . . . . . . 46

$4.3 \quad$ Numerical Checking of Derivatives . . . . . . . . . . . . . . . 50

$4.4 \quad$ Partial derivative of drag coefficient with respect to slip length . . . . . . 51

$4.5 \quad$ Partial derivative of pressure drop with respect to slip length . . . . . . . 51

$4.6 \quad$ Partial derivative of propeller efficiency with respect to slip length . . . . 52 
General Expression ..................... . . 53

NUMERICAL IMPLEMENTATION . . . . . . . . . . . . . 55

$5.1 \quad$ Optimization Flowchart . . . . . . . . . . . . . 55

$5.2 \quad$ Primal and Adjoint Solver . . . . . . . . . . . . . . . 55

$5.3 \quad$ Software Optimizer . . . . . . . . . . . . . . . . 56

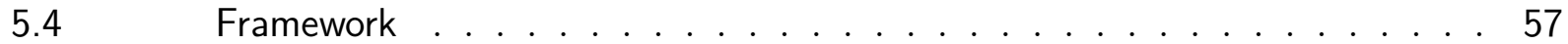

6 METHODS AND RESULTS FOR PROPELLER CFD SIMULATIONS 59

$6.1 \quad$ Propeller Geometry . . . . . . . . . . . . . . . . . . . . 59

6.2 Domain Geometry and Boundary Conditions . . . . . . . . . . . . . . . 60

6.3 Mesh Topology . . . . . . . . . . . . . . . . . . . . 63

$6.3 .1 \quad$ Mesh refines . . . . . . . . . . . . . . . . 63

$6.3 .2 \quad$ Mesh candidates . . . . . . . . . . . . . . . . 64

6.3 .3 Results and Verification . . . . . . . . . . . . . . . 66

6.3.3.1 Discretization Uncertainty . . . . . . . . . . . . . . . . 66

$6.3 .3 .2 \quad$ Computational costs . . . . . . . . . . . . . . . . 67

6.3.3.3 Curve of convergence . . . . . . . . . . . . . . 67

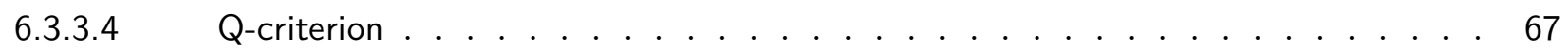

6.3 .3 .5 Discussion . . . . . . . . . . . . . . . . . . 68

$6.3 .4 \quad$ Mesh for Optimization Framework . . . . . . . . . . . . . . 68

$6.4 \quad$ Turbulence Model . . . . . . . . . . . . . . . . 70

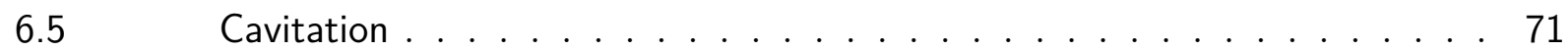

$6.6 \quad$ Experimental Values Comparative and Discussions . . . . . . . . . . . 72

7 NUMERICAL ANALYSIS AND RESULTS OF HYDROPHOBIC SIM-

ULATIONS . . . . . . . . . . . . . . . . . . . . 75

$7.1 \quad$ 2D Hydrofoil Comparatives . . . . . . . . . . . . . . . 75

7.1.1 Hydrofoil geometry . . . . . . . . . . . . . . . 75

7.1.2 Domain Geometry and Boundary Conditions . . . . . . . . . . . . . . . 75

7.1.3 Mesh Topology . . . . . . . . . . . . . . 76

$7.2 \quad$ Simulation conditions . . . . . . . . . . . . . . . 77

$7.3 \quad$ Hydrofoil Results . . . . . . . . . . . . . . . . . . 77

7.3.1 Lift and Drag Coefficients . . . . . . . . . . . . . . 77

7.3.2 Skin Friction Coefficient . . . . . . . . . . . . . . 78

$7.3 .3 \quad$ Pressure Coefficient . . . . . . . . . . . . . . 79

$7.4 \quad$ 3D Propeller Comparatives . . . . . . . . . . . . . . . . . . . 81

7.4.1 Results: Open Water Diagram . . . . . . . . . . . . . . . . 81

7.4.2 Skin Friction Coefficient . . . . . . . . . . . . . . . . . . 82

7.4.3 Pressure Coefficient . . . . . . . . . . . . . . . 82 
7.4.4 Segregated and Coupled Solvers . . . . . . . . . . . . . . . . 83

$7.5 \quad$ Propeller efficiency $-C p$ trade-off $\ldots \ldots \ldots \ldots$. . . . . . . . 85

$7.6 \quad$ Discussions . . . . . . . . . . . . . . . . . . 86

8 TOPOLOGY OPTIMIZATION RESULTS: TWO-DIMENSIONAL CASES ....................... . . 87

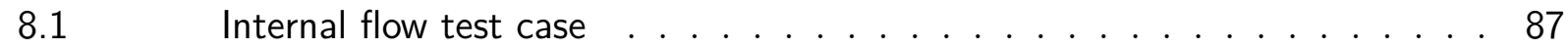

8.2 Sensitives comparison: Adjoint and Finite Difference Methods . . . . . . . . 88

$8.3 \quad$ Results of Topology Optimization . . . . . . . . . . . . . . . . . 89

8.3.1 Comparison with proposed topologies . . . . . . . . . . . . . . 89

8.3.2 Influence of slip length . . . . . . . . . . . . . . . . . 91

8.3.3 Influence of surface constraint . . . . . . . . . . . . . . . 92

8.3.4 Influence of Reynolds number . . . . . . . . . . . . . . . . . 95

8.3.5 Influence of angle of attack . . . . . . . . . . . . . . . 98

9 TOPOLOGY OPTIMIZATION RESULTS: PROPELLER CASE . . . 103

9.1 Topology of SHS distribution . . . . . . . . . . . . . . . . . . 103

$9.2 \quad$ Post-processing . . . . . . . . . . . . . . . . . . . 107

$9.3 \quad$ Finer mesh comparison . . . . . . . . . . . . . . . . . . 108

$9.4 \quad$ Comparative with no-SHS and full-SHS . . . . . . . . . . . 110

9.5 Discussions . . . . . . . . . . . . . . . . . . . . 111

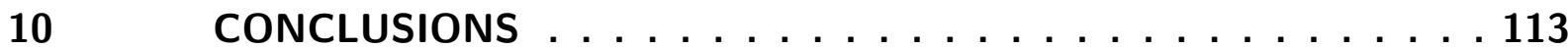

REFERENCES . . . . . . . . . . . . . . . 115

$\begin{array}{ll}\text { APPENDIX } & 123\end{array}$

APPENDIX A - FLUID TOPOLOGY OPTIMIZATION OF DOUBLE PIPE . . . . . . . . . . . . . . 125

A.1 Problem description . . . . . . . . . . . . . . . . 125

A.2 Stokes flow formulation . . . . . . . . . . . . . . . . . 126

A.3 Material model . . . . . . . . . . . . . . . . . 126

A.4 Optimization formulation . . . . . . . . . . . . . . . 127

A.5 Sensitivity comparison between Adjoint and Finite Difference methods . . . 127

A.6 Results and Comparison . . . . . . . . . . . . . . . 128

APPENDIX B - EXPERIMENTAL PROCEDURES - IPT'S CAVITATION TUNNEL VERIFICATION . . . . . . . 131

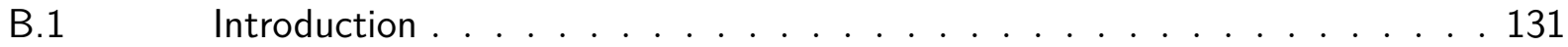




\begin{tabular}{|c|c|}
\hline B.2 & Theoretical Review of Uncertainty in Linear Regression \\
\hline B.3 & Propeller coefficients \\
\hline B.4 & Source of uncertainty \\
\hline B.5 & Measurement and Resolution uncertainties . . . . . . . . . . . 136 \\
\hline & Force and Moment Verification . . . . . . . \\
\hline B.6.1 & Thrust Expression and Uncertainty Expansion \\
\hline B.6.2 & Torque Expression and Uncertainty Expansion \\
\hline 6.3 & Load Cells . . . . . \\
\hline В.6.4 & Bearing Friction . . . . . \\
\hline B.7 & Advance Velocity Verification . . . . . . . . . . . . . . . . . . . . . . . . 141 \\
\hline 1 & Upstream Velocity Expression and Uncertainty Expansion . . . . . . . \\
\hline 7.2 & Pitot Tube Velocity Measurement . . . \\
\hline B.7.3 & Advance Velocity verification . . . . . . \\
\hline B.7.4 & Blockage ratio verification . . . . . . . . \\
\hline 8 & Rotating rate verification \\
\hline B.9 & Density and Vapor Pressure . . . . . \\
\hline 10 & Hydrostatic pressure . . \\
\hline B.11 & $\ldots \ldots 147$ \\
\hline & Cavitation Tunnel Uncertainty . . . . . \\
\hline & Turbulence intensity $\ldots \ldots \ldots$ \\
\hline
\end{tabular}

APPENDIX C - BLOCKAGE EFFECTS . . . . . . . . . . . 151

C.1 Introduction . . . . . . . . . . . . . . . . 151

C.2 Potential blockage correction . . . . . . . . . . . . . . 151

C.3 Domain Geometry and Boundary Conditions . . . . . . . . . . . . . . 152

C.4 Mesh Topology . . . . . . . . . . . . . . . . 153

C.5 Steady-state blockage results . . . . . . . . . . . . . . 154

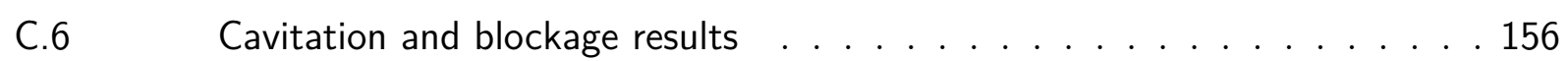

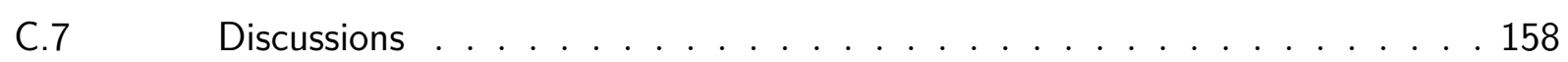




\section{Introduction}

In many practical applications in fluid mechanics, it is common to have the interest to reduce energy dissipation by reducing the drag or pressure drop. Especially after increased concern in the emission of greenhouse gases, save energy consumption by drag reduction is a way to reduce these emissions (BOUMAN et al., 2017). The increased knowledge in the emission estimates of greenhouse gases from ships stimulated the International Maritime Organization (IMO) to adopt an Energy Efficiency Design Index (EEDI) for the design of ships, being discussed in Longva, Eide and Skjong (2010) on how to determine the EEDI based on a cost-effective criterion. For several years, shipyards and research centers in the Naval Architecture and Oceanic Engineering area have studied the process of hull and propeller optimization to improve the efficiency of large vessels, obtaining high-end optimized hulls and propellers for each application. In order to increase the ship energy efficiency, research has been carried out on new technological solutions, such as the use of bubbles to reduce hull friction (MÄKIHARJU; CECCIO, 2018) or the use of technologies for energy absorption from the environment (SASAKI, 2011), among other novel technologies.

One of the main drawbacks of these technologies is that they depend on a new design of the vessel, being very difficult to be applied to an existing one. At this point, another emerging research field in which it is intended to reduce hydrodynamic resistance, i.e., improve performance, is about painting hulls and propellers with low friction paints, such as paints that create hydrophobic surfaces. The application of these paints make the hull a Super-Hydrophobic Surface (SHS). Thus, vessels could slightly increase their efficiency by just performing the proper paint maintenance (FU; YUAN; BAI, 2017).

In a recent report of ITTC (2017), it is indicated that there is an interest from industry and academia in high-efficiency vessels, by adopting frictional drag reduction methods. Riblets and textured surfaces, compliant surfaces, large-eddy breakup devices, SHS, and coatings/paints are cited as passive methods to frictional drag reduction methods. Mutton et al. (2005) presented a full-scale study, through a three-year follow-up of a vessel with and without anti-foil painting on the propeller. However, due to environmental implications, it was not possible to verify the performance difference, yet, the authors verified that the painted propeller presented a lower surface roughness than the propeller without the paint. Korkut and Atlar (2012) and Mutton et al. (2005) conducted an experimental study on a model-scale propeller in a Cavitation Tunnel comparing the observed cavitation patterns in propellers with and without commercial paint. A small improvement of the cavitation was verified in uniform and non-uniform (wake condition) flow conditions. 
However, there is a lack of research in SHS applied to propellers. The main effect of the hydrophobic painting is to change its friction drag, varying thrust, torque, and efficiency point. Therefore, an optimization study of the paint pattern with superhydrophobic properties is addressed in this work. A method for propeller simulations using Computational Fluid Dynamics (CFD) is required to simulate the propeller performance at a specific distribution of painting. Hydrophobicity is numerically analyzed in detail, and it is observed that there is a trade-off between increased efficiency and reduction of absolute pressure, which is undesirable since it can affect cavitation phenomena. Because of this, topology optimization, a candidate method for distributing hydrophobicity on surfaces, is introduced in the next sections.

\subsection{Super-Hydrophobic Surfaces - SHS}

SHS can be categorized by the contact angle formed with a water drop, as shown schematically in Fig. 1. These surfaces are commonly known to exhibit high repellency, being used in self-cleaning surfaces in several fields of engineering, from clothing (CHO et al., 2009), glasses (PARK et al., 2011) and even on cementitious composites for highway applications (MUZENSKI; FLORES-VIVIAN; SOBOLEV, 2015). Due to its self-cleaning behavior, Liu, Chen and Xin (2009) investigated whether surfaces repel hot water, concluding that it shows remarkably decreased repellency to hot water, which may be attributed to a decrease of surface tension.

Figure 1 - Classification through water drop contact angle.

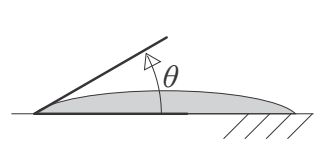

Hydrophilic $\theta<30^{\circ}$

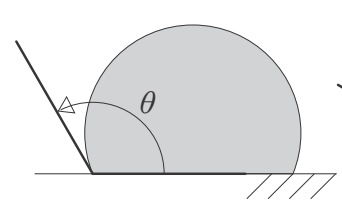

Hydrophobic $90^{\circ}<\theta<120^{\circ}$

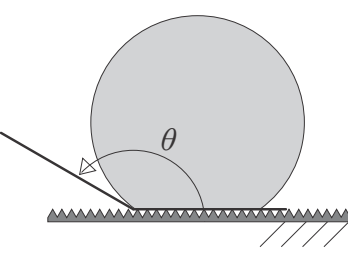

Overhydrophobic $120^{\circ}<\theta<150^{\circ}$

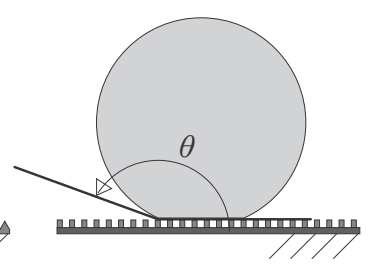

Super-hydrophobic $\theta>150^{\circ}$

Source: Adapted from Muzenski, Flores-Vivian and Sobolev (2015)

SHS is not purely synthetic, being present in nature, such as in a lotus leaf, Fig. 2. It can be specially observed after raining on these leaves, with water beads with a contact angle which defines lotus leaves as super-hydrophobic. Cheng and Rodak (2005) studied the conditions on which a lotus leaf is considered hydrophobic.

Super-hydrophobic surface studies have intensified in recent years. Ou, Perot and Rothstein (2004) investigated the effect of drag reduction in laminar flow, comparing the numerical model with experimental results. Voronov, Papavassiliou and Lee (2006), Voronov, Papavassiliou and Lee (2007), Voronov, Papavassiliou and Lee (2008) studied 
Figure 2 - Optical photos of cool water $\left(25^{\circ} \mathrm{C}\right.$, a $)$ and hot water $\left(55^{\circ} \mathrm{C}\right.$, b) on lotus leaves; images of hydrophobic lotus leaf surface $(\mathrm{c}$, bar $=10 \mu \mathrm{m})$ and $55^{\circ} \mathrm{C}$ hot water washed lotus leaf surface $(\mathrm{d}$, bar $=10 \mu \mathrm{m})$
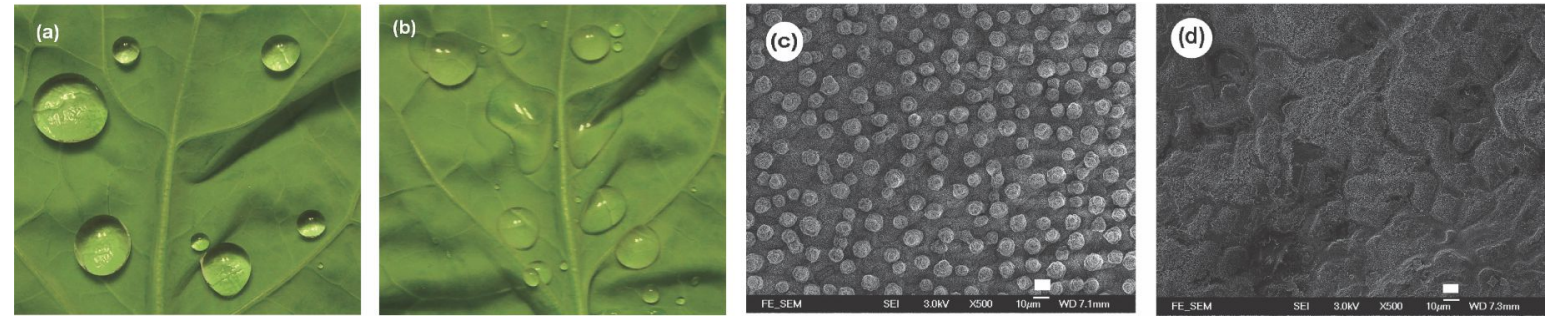

Source: Liu, Chen and Xin (2009)

the characteristics of hydrophobicity, aiming to investigate the relationship between the contact angle and slip length (variable that defines the super-hydrophobicity).

Super-Hydrophobic (SH) studies were being led successful for laminar flow, stimulating researchers to start the phenomena exploration in the turbulent flow context. According to Seo and Mani (2016), the main difference between laminar and turbulent flows is the separation of scale between the length scale of the mean velocity gradients and that of macroscopic geometry. A shifted-turbulent boundary layer model was proposed to simulate the behavior on hydrophobic surfaces. The relationship between the slip length the microstructure hole was obtained, being analyzed the condition in which this relationship is valid.

The work of Min and Kim (2004) is one of the first to investigate the effects of SHS using Direct Numerical Simulation (DNS) with turbulence phenomena. It is observed that the SHS effect reduces the drag in the same direction of the flow and increases it in the case of perpendicular flow. Additionally, Daniello, Waterhouse and Rothstein (2009) investigated the effect in turbulent flows in a marine vessel with an experimental approach, observing significant drag reduction by Particle Image Velocimetry (PIV) and direct pressure measurements.

Some works are based on creating an SHS with a sprayable coating, due to its easy application. Wang et al. (2016) studied the $\mathrm{pH}$ and waterfall/jet effects in a commercial super-hydrophobic spray. The authors concluded that the waterfall could lead to the loss of super-hydrophobicity of the coating, induced by loss of nanoparticles on the SHS.

The magnitude of slip velocity and the effect of the SHS on Reynolds stress in the inner and outer layers have been experimentally investigated using simultaneous long-range micro Particle Tracking Velocimetry (PTV) and macroscopic PIV (ROWIN; HOU; GHAEMI, 2017). The analysis on turbulence statistics reflects the variations in physical dimensions if the measured Reynolds stresses are normalized using a common inner scaling. 
Gao, Liu and $\mathrm{Hu}$ (2017) studied the effect of applying super-hydrophobic paint to an Unmanned Aerial Systems (UAS) propeller in order to analyze the consequence in the formation of ice. A detachment of the paint was observed over time, showing that for highly turbulent conditions, it is difficult to maintain a sprayable super-hydrophobic paint. As observed, the SHS study is present in the aeronautical field of study as well.

\subsection{Optimization Methods}

The optimization process consists in guiding to the best possible solution on a design domain, based on a cost function, through design variables delimited by a feasible interval and their constraint. In this work, the design domain is the blade surface, and, due to its periodicity, the study is carried on only one blade to save computational cost, as shown in Fig. 3.

Figure 3 - Representation of study domain based on one blade
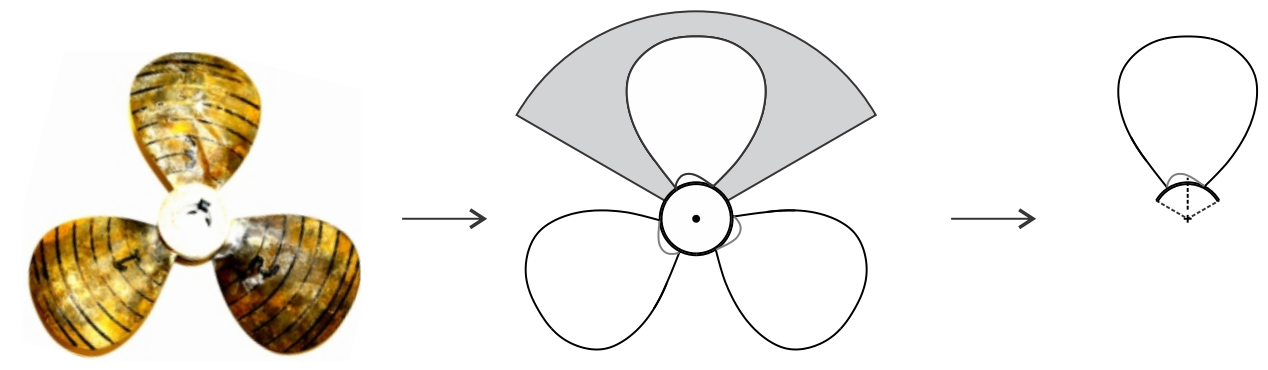

Source: Author

The optimization can be divided into three main types: parametric, shape and topology optimization, shown in Fig. 4.

Figure 4 - Parametric (left); shape (center); and topology optimization (right) applied to surface painting optimization
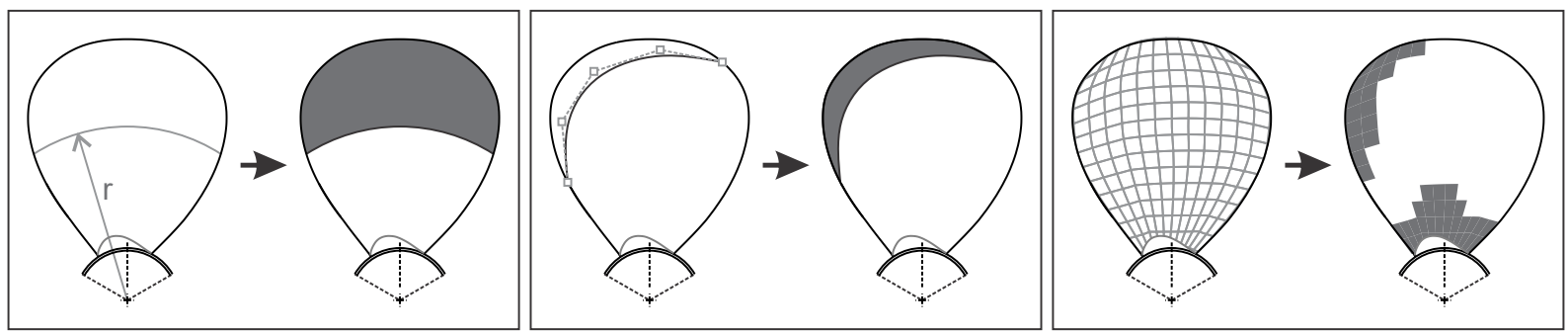

Source: Author

In parametric optimization, the characteristics to be optimized are defined in terms of dimensions, which are changed and computed through a cost function, guiding to an 
optimized solution. For the problem of defining a paint region, an example of a dimension that could be used is the radius at which the paint begins to be applied. Note that the ability of the generalization of the paint region depends on how detailed regions are and, consequently, on the constituent dimension numbers.

The shape optimization is based on curve manipulation by defining a form in which it contains the domain of interest. It should have an initial idea of the curve shape to performing a consistent parametrization. The curve shape can be defined through a B-spline function that defines the domain where to apply the painting. As the same disadvantage as parametric optimization, shape optimization is able to define a generic region as much as its generalization for curve generation. Some regions may not be considered in the optimization process due to poor discretization.

Finally, topology optimization has the advantage of generality, not depending on a proper initial guess, as stated in the previous methods. Through a mesh in which each element can have the characteristic of pseudo-density ranging from 0 to 1 , the optimized region is defined. This is a more general approach since it allows the material to be freely distributed in the field. In this work, the blade is discretized into elements and, having the presence of painting in certain regions.

The comparative analysis of three types of optimization showed that the topology optimization, hypothetically, can have several pieces of regions: the presence of the painting is found near the axis and at the end of the blade. Therefore, the Topology Optimization Method (TOM) is a generic tool that allows finding new alternatives, being the adopted method.

The generality provided by the method came on to academia in the 1980s in the United States and Europe with the publication of Bendsøe and Kikuchi (1988) as an application in solid mechanics. In the 1990s, it became widely used in the automotive and aeronautical industries of the United States, Japan, and Europe for the design of optimized mechanical parts. Its application has expanded to heat conduction problems (GERSBORGHANSEN; BENDSØE; SIGMUND, 2006), fluid mechanics of rotor (ROMERO; SILVA, 2014) or applied to surfaces, as in irradiation studies (CASTRO; KIYONO; SILVA, 2015).

\subsection{Motivation}

There is concern about environmental impact and efficiency on large vessels. A more efficient marine propulsion system reduces this impact and contributes to the cost reduction of naval shipping.

Hydrophobic paints are usually not cheap and should be applied according to established criteria. Other side effects may arise with their application, which should be 
further investigated.

The development of topology optimization applied to fluids has grown, and its implementation in Finite Volume Method (FVM) with turbulence models can be extended to other applications, such as the roughness distribution for a laminar-turbulent transition control.

\subsection{Objectives}

This work has the main objective of optimizing the pattern distribution of paint with super-hydrophobic properties on the surface of a model-scale marine propeller, with existing defined geometry, using TOM, in order to increase the hydrodynamic efficiency.

In order to accomplish this objective, a method for propeller simulations using CFD is developed, comparing results with experimental tests. Then, a study of super-hydrophobic surfaces hydrodynamics is done. Finally, TOM is used to design the distribution of the application of the SH painting. It is not the scope of this work to discuss the formulation of the super-hydrophobic model nor validate SHS with experiments. The formulation is based on the previous formulation presented in the literature.

\subsection{Document outline}

This document is organized as follows: In Sec. 2, the theoretical formulation of basic concepts of marine propellers, fluid mechanics and numerical methods are shown. Topology Optimization is presented in more detail in Sec. 3, with details about the method and its requirements, showing the importance of the sensitivity and presenting methods to obtain it, through the adjoint method. In the following session, Sec. 4, the discrete adjoint formulation applied to hydrophobicity is deduced. In Sec. 5, the numerical implementation and the framework of the optimization are presented. Section 6 shows the method development for propeller simulations using CFD, with comparative with experimental results. Once the method is defined, Sec. 7 presents a numerical investigation of the hydrophobic model. Since performing topology optimization using 3D CFD simulations is computationally expensive, Sec. 8 presents a preliminary step: it presents the topology optimization of pattern distribution of paint with SH properties in 2D model cases. Finally, Sec. 9 shows the results of topology optimization on the propeller blade.

The NASA's document about the future of CFD through the year 2030 (SLOTNICK et al., 2014) shows that the basic set of capabilities for Vision 2030 CFD must include errors and uncertainties estimates. In alignment with this objective, the present document shows the estimate of errors during its processes whenever possible. The detailing of the experimental error, for example, is developed in Appendix B. It is also interesting to point 
out that the document also cites CFD as a step towards Multidisciplinary Analysis and Optimization (MDAO). Although this thesis is not in a multidisciplinary context, the material developed with adjoint methods contributes to use CFD (which traditionally uses FVM) as a tool for MDAO. 



\section{Theoretical Formulation}

\subsection{Basic Concepts of Marine Propellers}

The concepts about marine propellers presented here are based on Carlton (2012). The advance ratio $J$, defined in Eq. (2.1), is a non-dimensional number that relates the advance velocity $v_{a}$ with the propeller diameter $D_{p}$ and its rotation $n$, in revolution per second.

$$
J=\frac{v_{a}}{n D_{p}}
$$

Thrust $T$ and required torque $Q$ are related with non-dimensional numbers: Thrust coefficient $K T$ and torque coefficient $K Q$, and given for open-water condition by Eq. (2.2) and Eq. (2.3), respectively.

$$
\begin{aligned}
& K T=\frac{T}{\rho n^{2} D_{p}^{4}} \\
& K Q=\frac{Q}{\rho n^{2} D_{p}^{5}}
\end{aligned}
$$

in which $\rho$ is the fluid density.

The hydrodynamic efficiency of propeller $\eta$ is defined as the ratio of propeller power and the engine power for propeller rotation:

$$
\eta=\frac{T v_{a}}{Q 2 \pi n}=\frac{J}{2 \pi} \frac{K T}{K Q}
$$

The Reynolds number for propeller can be defined as:

$$
R e_{p}=\frac{\rho n D_{p}^{2}}{\mu}
$$

in which $\mu$ is dynamic viscosity. The Reynolds number for foils is defined as:

$$
R e_{x}=\frac{\rho v_{a} \ell}{\mu}
$$

in which $\ell$ is a characteristic length, such as foil's chord or the pipe width. 
The cavitation number can be defined as:

$$
\sigma=\frac{p-p_{s}}{\frac{1}{2} \rho\left(n D_{p}\right)^{2}}
$$

in which $p_{s}$ is the saturation pressure.

\subsection{Fluid Flow Fundamental Equations}

Basic concepts of fluid mechanics are presented, available in more details in Versteeg and Malalasekera (2007) or Anderson (1995).

The main fluid of this work is water. The fundamental equations are simplified, considering incompressibility and constant Newtonian viscosity, valid assumptions for water (CARLTON, 2012). In the following equations, $\Omega$ denotes the volume control; $\partial \Omega$, surface of the volume control; $\mathbf{u}$, velocity; $p$, pressure; and $\mathbf{s}$, vector area.

The equation of continuity represents the physical principle that mass can not be created or destroyed. The application of the Reynolds transport theorem from this principle to a generic fixed control volume and its respective control surface results in a continuity equation, being presented in differential form in Eq. (2.8), and in integral form in Eq. (2.9).

$$
\begin{gathered}
\nabla \cdot \mathbf{u}=0 \\
\oint_{\partial \Omega} \mathbf{u} \cdot d \mathbf{s}=0
\end{gathered}
$$

in which $\nabla$ is the nabla operator (and $\nabla \cdot$, the divergence operator).

The equation of momentum represents the application of Newton's second law applied to fluid mechanics. According to it, the sum of the external forces is equal to the variation of linear momentum. Applying the Reynolds transport theorem to a generic volume, Eq. (2.10), also called Navier-Stokes momentum equation, is obtained.

$$
\rho\left[\frac{\partial \mathbf{u}}{\partial t}+(\mathbf{u} \cdot \nabla) \mathbf{u}\right]=-\nabla p+\nabla \cdot \mathbf{T}+\mathbf{f}_{\mathbf{b}}
$$

in which $\mathbf{f}_{\mathbf{b}}$ denotes the resultant of body forces per volume. The shear stress tensor $\mathbf{T}$ is given by Eq. (2.11).

$$
\mathbf{T}=2 \mu \mathbf{D}+\zeta(\nabla \cdot \mathbf{u}) \mathbf{I}
$$


in which $\zeta$ is the Lamé coefficient (also known as second coefficient of viscosity). With the Stokes hypothesis, $2 \mu+3 \zeta=0$, it can be rewritten as $\zeta=-2 / 3 \mu$. However, since it is assumed incompressible flows, the second Right-Hand Side (RHS) term of Eq. (2.11) is zero.

The rate of deformation (strain) tensor is given by Eq. (2.12).

$$
\mathbf{D}=\frac{1}{2}\left[\nabla \mathbf{u}+(\nabla \mathbf{u})^{T}\right]
$$

Replacing Eq. (2.11) into Eq. (2.10) results in Eq. (2.13).

$$
\rho\left[\frac{\partial \mathbf{u}}{\partial t}+(\mathbf{u} \cdot \nabla) \mathbf{u}\right]=-\nabla p+\nabla \cdot(2 \mu \mathbf{D})+\mathbf{f}_{\mathbf{b}}
$$

Expanding the second RHS term results in Eq. (2.14).

$$
\nabla \cdot(2 \mu \mathbf{D})=\mu\left[\nabla^{2} \mathbf{u}+\nabla(\nabla \cdot \mathbf{u})\right]
$$

Note again that if considering the continuity equation, Eq. (2.8), into Eq. (2.14), the momentum equation results in Eq. (2.15).

$$
\rho\left[\frac{\partial \mathbf{u}}{\partial t}+(\mathbf{u} \cdot \nabla) \mathbf{u}\right]=-\nabla p+\mu \nabla^{2} \mathbf{u}+\mathbf{f}_{\mathbf{b}}
$$

Another form of momentum equation is presented in Eq. (2.16), using the Kronecker product.

$$
\rho\left[\frac{\partial \mathbf{u}}{\partial t}+\nabla \cdot(\mathbf{u} \otimes \mathbf{u})\right]=-\nabla p+\mu \nabla^{2} \mathbf{u}+\mathbf{f}_{\mathbf{b}}
$$

Equation (2.10), Eq. (2.15) and Eq. (2.16) are usual differencial forms of momentum equation for a incompressible flow. The momentum equation in integral form is presented in Eq. (2.17).

$$
\frac{\partial}{\partial t} \int_{\Omega} \rho \mathbf{u} d \Omega+\oint_{\partial \Omega} \rho \mathbf{u} \otimes \mathbf{u} \cdot d \mathbf{s}=-\oint_{\partial \Omega} p \mathbf{I} \cdot d \mathbf{s}+\oint_{\partial \Omega} \mathbf{T} \cdot d \mathbf{s}+\int_{\Omega} \mathbf{f}_{\mathbf{b}} d \Omega
$$

in which $\mathbf{I}$ is the identity tensor.

\subsection{Moving Reference Frame - MRF}

A Moving Reference Frame (MRF) approach is used to consider the propeller rotation in steady-state simulation. In this, governing equations are solved in the MRF, but its formulation is written in a fixed (absolute) reference frame. 
Since only the rotation is interested in this work (the translation term is disregarded), the relative velocity in a material point can be written as:

$$
\mathbf{u}_{\mathbf{r}}=\mathbf{u}-\boldsymbol{\omega} \times \mathbf{r}
$$

in which $\boldsymbol{\omega}$ is the angular velocity vector and $\mathbf{r}$, distance vector between origin and material point.

Equation (2.17), in terms of the fluid velocity in absolute velocity, is written as:

$$
\frac{\partial}{\partial t} \int_{\Omega} \rho \mathbf{u} d \Omega+\oint_{\partial \Omega} \rho \mathbf{u} \otimes \mathbf{u}_{\mathbf{r}} \cdot d \mathbf{s}+\int_{\Omega} \rho \boldsymbol{\omega} \times \mathbf{u} d \Omega=-\oint_{\partial \Omega} p \mathbf{I} \cdot d \mathbf{s}+\oint_{\partial \Omega} \mathbf{T} \cdot d \mathbf{s}+\int_{\Omega} \mathbf{f}_{\mathbf{b}} d \Omega
$$

Note that a volumetric term is added in the Left-Hand Side (LHS) of the formulation, which represents the Coriolis and centripetal accelerations.

\subsection{Turbulence modeling}

Concepts of turbulence and its modeling are presented, based in books, such as Versteeg and Malalasekera (2007), Siemens (2017) and Pope (2000).

Flows are divided into two large groups: laminar and turbulent (and the transition between them). Turbulent flow is present in most practical applications, such as around cars, airplanes, water pipes, etc. The main characteristics of turbulent flow are its threedimensional and unsteady behavior; the chaotic and irregular trajectory of the particles of the fluid; strong diffusion and dissipation; and high Reynolds number values.

One approach to model a turbulence flow is to divide a variable in two parts: the averaged value $\bar{\phi}$ and fluctuating component $\phi^{\prime}$, as shown in Eq.(2.20).

$$
\phi=\bar{\phi}+\phi^{\prime}
$$

This approach is used to model turbulence through Reynolds-Averaged NavierStokes (RANS). Applying the averaged and fluctuating parts, Eq. (2.20), into the fluid flow governing equations of the fluid, Eq. (2.8) and Eq. (2.10), results in Eq. (2.21) and Eq. (2.22), respectively.

$$
\begin{gathered}
\nabla \cdot \overline{\mathbf{u}}=0 \\
\rho\left[\frac{\partial \overline{\mathbf{u}}}{\partial t}+(\overline{\mathbf{u}} \cdot \nabla) \overline{\mathbf{u}}\right]=-\nabla \bar{P}+\nabla \cdot\left(\mathbf{T}+\mathbf{T}_{\mathbf{t}}\right)+\mathbf{f}_{\mathbf{b}}
\end{gathered}
$$


in which $\overline{\mathbf{u}}$ is the average vector of velocity and $\bar{P}$, the average value of pressure.

$\mathbf{T}_{\mathbf{t}}$ is called Reynolds Stress Tensor, given by:

$$
\mathbf{T}_{\mathbf{t}}=-\rho\left[\begin{array}{lll}
\overline{u^{\prime 2}} & \overline{u^{\prime} v^{\prime}} & \overline{u^{\prime} w^{\prime}} \\
\overline{v^{\prime} u^{\prime}} & \overline{v^{\prime 2}} & \overline{v^{\prime} w^{\prime}} \\
\overline{w^{\prime} u^{\prime}} & \overline{w^{\prime} v^{\prime}} & \overline{w^{\prime 2}}
\end{array}\right]
$$

Boussinesq proposed that Reynolds Stress Tensor could be linked to mean rates of deformation, similarly with laminar flow (see the similarity with Eq. (2.11)), introducing the concept of eddy (or turbulent) viscosity $\mu_{t}$ :

$$
\mathbf{T}_{\mathbf{t}}=2 \mu_{t} \mathbf{D}-\frac{2}{3} \mu_{t}(\nabla \cdot \overline{\mathbf{u}}) \mathbf{I}-\frac{2}{3} \rho k \mathbf{I}
$$

Note that the Boussinesq hypothesis assumes that turbulent viscosity is isotropic, the same in all directions. Considering the continuity equation for turbulent flow and after some manipulation, RANS equations can be written as:

$$
\rho\left[\frac{\partial \overline{\mathbf{u}}}{\partial t}+(\overline{\mathbf{u}} \cdot \nabla) \overline{\mathbf{u}}\right]=-\nabla \bar{p}+\left(\mu+\mu_{t}\right) \nabla^{2} \overline{\mathbf{u}}+\mathbf{f}_{\text {ext }}
$$

in which $\bar{p}$ is the working pressure, defined as $\bar{p}=\bar{P}+2 / 3 \rho k$, including also the dynamic pressure associated to the eddies.

Turbulent kinetic energy is defined as:

$$
k=\frac{1}{2}\left(\overline{u^{\prime 2}}+\overline{v^{\prime 2}}+\overline{w^{\prime 2}}\right)
$$

Turbulence intensity is given by:

$$
T_{i}=\frac{\sqrt{\frac{2}{3} k}}{U_{r e f}}
$$

The reference velocity can be given by:

$$
U_{r e f}=\sqrt{\bar{u}^{2}+\bar{v}^{2}+\bar{w}^{2}}
$$

Many turbulence models are based upon the RANS-Boussinesq hypothesis, in which three of them are chosen and detailed.

Realizable $k-\varepsilon$ model (SHIH et al., 1995) is chosen instead of traditional $k-\varepsilon$ model (LAUNDER; SPALDING, 1974) due to Star-CCM+ CFD developer recommendation (Siemens, 2017), claiming that the Realizable model is better for many applications and 
that produces results at least as accurate as the traditional one. The treatment of the kinetic turbulence model is the same for both, being different in the formulation of dissipation and turbulent viscosity.

The $k-\omega$ SST model (MENTER, 1994), uses the traditional $k-\omega$ model formulation (WILCOX, 1993) near to the wall and $k-\varepsilon$ formulation far from the wall, avoiding the high sensibility of $k-\omega$ model in the inlet regions of free-stream. As Realizable $k-\varepsilon$, the $k-\omega$ SST model is recommended instead of the traditional model. Also, according to Versteeg and Malalasekera (2007), $k-\omega$ SST model is the most general, and tests suggest that it gives superior performance for zero pressure gradient and adverse pressure gradient boundary layers, free shear layers and a NACA4412 airfoil.

The Spalart-Allmaras model (SPALART; ALLMARAS, 1992), developed to aerospace applications, is the only model among the ones mentioned above to have just one transport equation to model the Reynolds stress, through the turbulent viscosity that relates the turbulent kinetic energy and the dissipation.

\subsection{Boundary Conditions in CFD simulations}

The numerical treatment for velocity inlet boundary conditions is that the velocity is specified, pressure gradient is zero, and turbulence kinetic energy and specific dissipation rate (or turbulent dissipation rate, for $k-\varepsilon$ based models) are also specified. For pressure outlet boundary condition, velocity gradient is zero, pressure is imposed to be zero, turbulent kinetic energy and specific dissipation rate (or turbulent dissipation rate, for $k-\varepsilon$ based models) gradients are zero. The design surface is modeled as a wall boundary condition: velocity is imposed (tangential velocity to represent an SHS), pressure and turbulent kinetic energy gradients are zero and specific dissipation rate has a specified value. In the propeller simulations, a periodic condition defines two part surfaces that are spatially detached, but the rotation rate can make them coincident.

\subsection{Wall treatment}

The dimensionless wall distance $y^{+}$and dimensionless velocity $u^{+}$are defined below:

$$
y^{+}=\frac{\rho u^{*} y}{\mu} \quad u^{+}=\frac{u}{u^{*}}
$$

in which $u^{*}=\sqrt{|\boldsymbol{\tau}| / \rho}$ is the friction velocity; $u$, velocity parallel to wall; $|\boldsymbol{\tau}|$, wall shear stress magnitude; and $y$, distance normal to the wall.

In the turbulent boundary layer, flow can be divided into three parts: the viscous sub-layer, the buffer layer, and the log-law region. The modeling for viscous sub-layer and 
log-law velocities can be given by:

$$
u_{\mathrm{visc}}^{+}=y^{+} \quad u_{\log }^{+}=\frac{1}{\kappa} \ln \left(\frac{E}{f} y^{+}\right)
$$

The blending of these model, used for all $y^{+}$wall treatment model and described in Siemens (2017), is given by:

$$
u^{+}=\frac{1}{\kappa} \ln \left(1+\kappa y^{+}\right)+a_{1}\left[1-\exp \left(-\frac{y^{+}}{a_{2}}\right)-\frac{y^{+}}{a_{2}} \exp \left(-a_{3} y^{+}\right)\right]
$$

in which each component is given below.

$$
a_{1}=\frac{1}{\kappa} \ln \left(\frac{E}{f \kappa}\right) \quad a_{2}=y_{m}^{+} \quad a_{3}=\frac{1}{2}\left(\frac{D k}{C}+\frac{1}{D}\right)
$$

Figure 5 shows the comparative of the parts for default values: $\kappa=0.42, E=9.0$ and $f=1.0$.

Figure 5 - Blended Wall Laws used in wall treatment for turbulent simulations

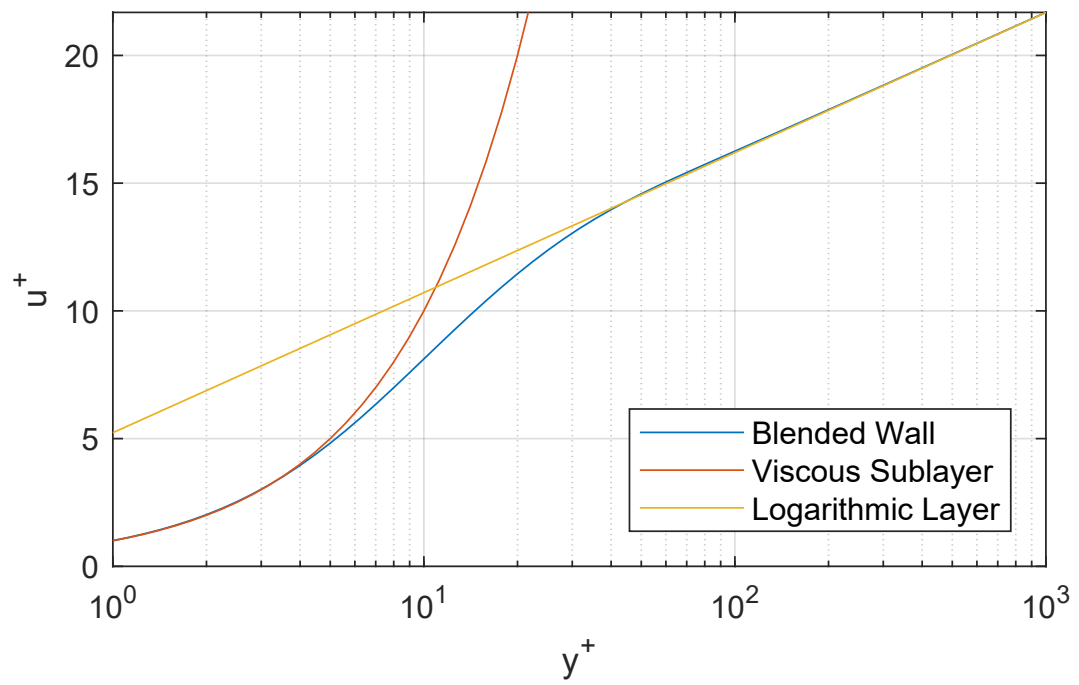

Source: Author

\subsection{Cavitation modeling}

The cavitation model is based on the Rayleigh-Plesset equation (BRENNEN, 1995), Eq. (2.33), and uses the inertia controlled growth model for cavitation bubble formation, (Siemens, 2017).

$$
\frac{p_{B}-p_{\infty}}{\rho_{l}}=R \frac{d^{2} R}{d t^{2}}+\frac{3}{2}\left(\frac{d R}{d t}\right)^{2}+\frac{4 \nu_{l}}{R} \frac{d R}{d t}+\frac{2 \sigma}{\rho_{l} R}
$$


In Eq. (2.33), $p_{B}$ is the pressure in the bubble, $p_{\infty}$ is the pressure of the surrounding liquid, $\rho_{l}$ is liquid density, $R$ is the bubble radius, $\nu_{l}$ is the kinematic viscosity and $\sigma$ is the surface tension coefficient.

\subsection{Super-hydrophobic modeling}

The micro-texture of an SHS is shown in Fig. 6. Due to water surface tension combined with periodic micro-surface elevations, SHS traps small air pockets in nano-scale pores, creating an interface between the liquid and solid substrate. This phenomenon creates, in macro-scale, an appearance that the fluid slips, presenting a reduced skin-friction drag.

Figure 6 - Schematic of the micro-structure of an SHS

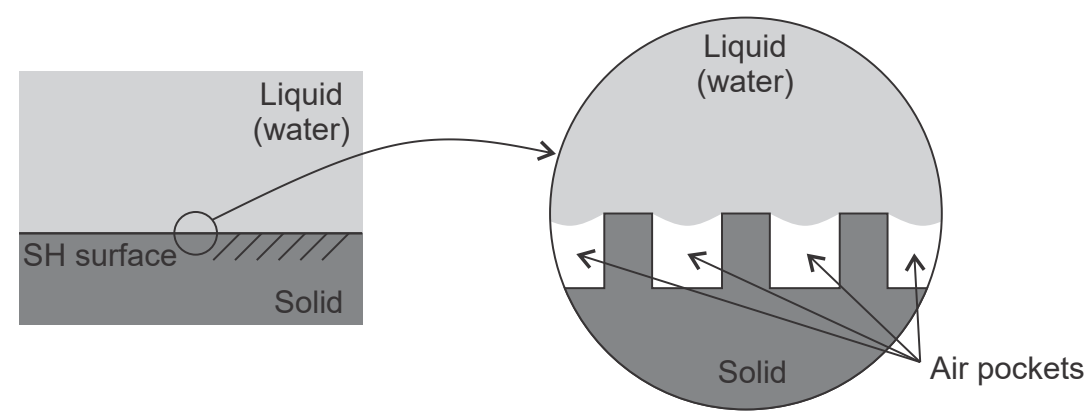

Source: Adapted from Ou, Perot and Rothstein (2004)

Figure 7 shows the flow velocity profile over an SHS. The fluid appears to slip over the surface, with a superficial velocity $u_{s}=u(0) \neq 0$ and appearing to have its no-slip point in a position $b$ below.

Figure 7 - Schematic of apparent velocity profile due to SHS

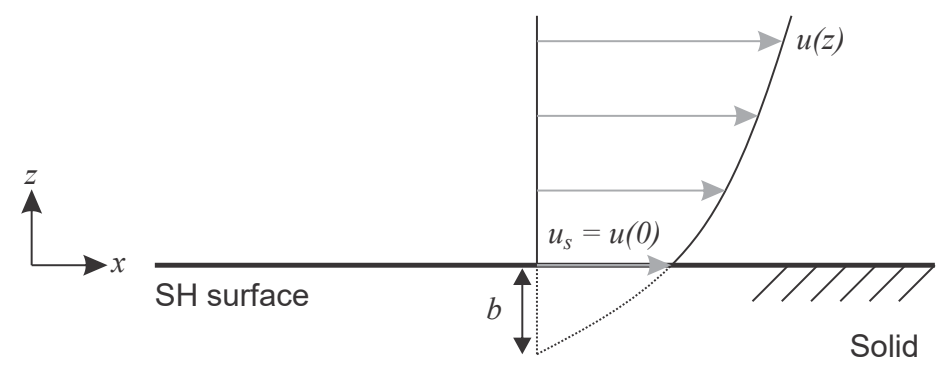

Source: Adapted from Ou, Perot and Rothstein (2004)

The SHS modeling is given by a tangential velocity proportional to velocity derivative in relation to the normal of the wall, that is:

$$
\mathbf{u}=b \frac{\partial \mathbf{u}}{\partial \mathbf{n}}
$$


This is a well-established relationship in studies of hydrophobia, available in articles such as in Min and Kim (2004), Daniello, Waterhouse and Rothstein (2009) and Seo and Mani (2016).

\subsection{Finite Volume Method - FVM}

The FVM is presented in detail by Patankar (1980) and Versteeg and Malalasekera (2007). FVM is one method used for solving Partial Differential Equations (PDE) in the discrete domain. According to Bakker (2006), 80\% of commercial available CFD programs uses FVM, while 15\% works with Finite Element Method (FEM). The method consists in integrating the differential equation over the control volume and apply the divergence theorem. After applying the solving process to obtain the values at the cell center, it needs values at control volume faces to obtain derivative terms. Then, the method is combined with interpolation techniques to estimate properties on the faces, such as high order or upwind methods to weight the interpolation. After evaluating all the elements and applying the boundary conditions, the result is a set of linear algebraic equations, one for each control volume.

Compared to the FEM, FVM presents some advantages, such as less memory usage and usually has a higher speed for very large problems, high speed and turbulent flows. Moreover, the Control Volume balance is not limited by cell shape. Mass and Momentum are conserved even on coarse meshes. On the other hand, it presents false diffusion when simple numerics are used.

An implementation example is presented by Bakker (2006) and Versteeg and Malalasekera (2007), and reproduced in the following.

The formulation of FVM for steady-state convection-diffusion problems is formulated below:

$$
\nabla \cdot(\phi \mathbf{u})=\nabla \cdot(\Gamma \nabla \phi)+g
$$

in which $\phi$ is a general property; $\Gamma$, the diffusion coefficient; and $g$, a source term.

The solution domain is subdivided into a finite number of small control volumes (elements), as shown in Fig. 8. The values at faces are not available and are determined by interpolation.

Integrating Eq. (2.35) for the $\Omega$ volume of element yields:

$$
\int_{\Omega} \nabla \cdot(\phi \mathbf{u}) d V=\int_{\Omega} \nabla \cdot(\Gamma \nabla \phi) d V+\int_{\Omega} g d V
$$


Figure 8 - Finite Volume Method schema

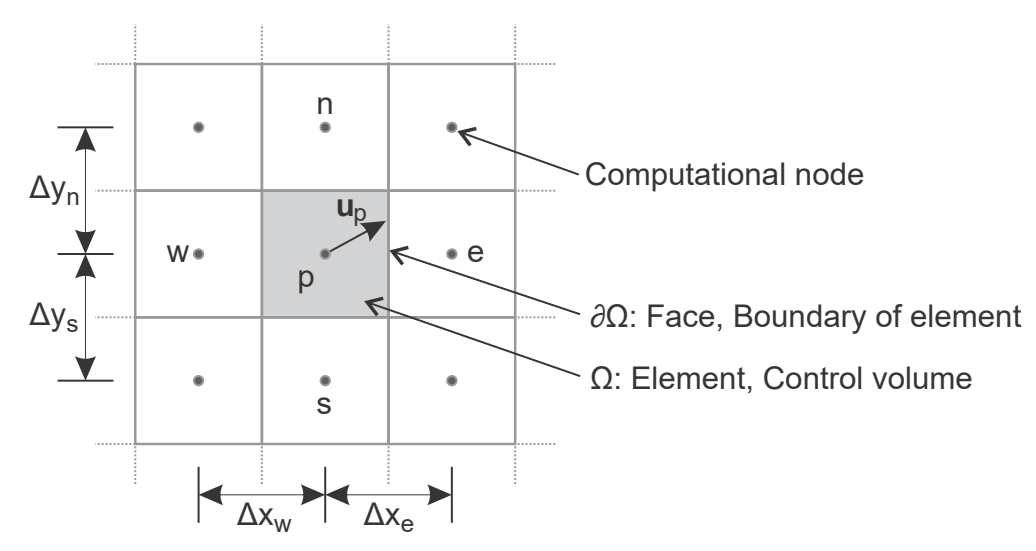

Source: Author

Applying the Divergence theorem:

$$
\oint_{\partial \Omega}(\phi \mathbf{u}) \cdot \mathbf{n} d s=\oint_{\partial \Omega}(\Gamma \nabla \phi) \cdot \mathbf{n} d s+\int_{\Omega} g d V
$$

Splitting the surface integrals in four, for each face of the volume:

$$
\begin{aligned}
& S_{e} u_{e} \phi_{e}-S_{w} u_{w} \phi_{w}+S_{n} u_{n} \phi_{n}-S_{s} u_{s} \phi_{s}= \\
&\left.\Gamma S_{e} \frac{d \phi}{d x}\right|_{e}-\left.\Gamma S_{w} \frac{d \phi}{d x}\right|_{w}+\left.\Gamma S_{n} \frac{d \phi}{d y}\right|_{n}-\left.\Gamma S_{s} \frac{d \phi}{d y}\right|_{s}+G
\end{aligned}
$$

in which $S$ is the face area and $u$ is the velocity (evaluated at the faces).

Since the values of velocity on faces are not available, they must be obtained through an interpolation method. A simple way is by the first-order upwind differencing: it is assumed that the value on the face is equal to the value in the center of the cell upstream of the face. Also, considering that a first-order differentiation approximates the value of the derivative is given by:

$$
\begin{gathered}
S_{e} v_{p} \phi_{p}-S_{w} v_{w} \phi_{w}+S_{n} v_{p} \phi_{n}-S_{s} v_{s} \phi_{s}= \\
\Gamma S_{e} \frac{\left(\phi_{e}-\phi_{p}\right)}{\Delta x_{e}}-\Gamma S_{w} \frac{\left(\phi_{p}-\phi_{w}\right)}{\Delta x_{w}}+\Gamma S_{n} \frac{\left(\phi_{n}-\phi_{p}\right)}{\Delta y_{n}}-\Gamma S_{s} \frac{\left(\phi_{p}-\phi_{s}\right)}{\Delta y_{s}}+G
\end{gathered}
$$

in which $v$ is the velocity (evaluated at the node). Rearranging to get an expression in 
terms of $\phi$ :

$$
\begin{aligned}
& \phi_{p} \underbrace{\left(S_{n} v_{p}+S_{e} v_{p}+\Gamma \frac{S_{w}}{\Delta x_{w}}+\Gamma \frac{S_{n}}{\Delta y_{p}}+\Gamma \frac{S_{e}}{\Delta x_{e}}+\Gamma \frac{S_{s}}{\Delta y_{s}}\right)}_{c_{p}}= \\
& \phi_{w} \underbrace{\left(S_{w} v_{w}+\Gamma \frac{S_{w}}{\Delta x_{w}}\right)}_{c_{w}}+\phi_{n} \underbrace{\left(\Gamma \frac{S_{n}}{\Delta y_{n}}\right)}_{c_{n}}+\phi_{e} \underbrace{\left(\Gamma \frac{S_{e}}{\Delta x_{e}}\right)}_{c_{e}}+\phi_{s} \underbrace{\left(S_{s} v_{s}+\Gamma \frac{S_{s}}{\Delta y_{s}}\right)}_{c_{s}}+G
\end{aligned}
$$

This equation can be simplified to:

$$
c_{p} \phi_{p}-c_{w} \phi_{w}+c_{n} \phi_{n}+c_{e} \phi_{e}+c_{s} \phi_{s}=G
$$

This equation shows a linear relationship between the variable of interests and constants. Performing the same procedure for all elements and considering boundary conditions, it results in a linear system that provides the value of these variables.

\subsection{Segregated and Coupled Flow Solvers}

Two solvers are discussed in this work to solve the Continuity and Navier-Stokes equations to get pressure and velocities fields.

The segregated flow solver deals with the governing equation iteratively, one equation at each time. For incompressible flows, density is assumed constant, and the Continuity equation does not present the pressure term. Even with four equations and four variables, which closes the formulation, there is a lack of connection between equations due to the link absence of pressure in Continuity. To tackle this problem, the so-called pressure-velocity coupling algorithms must be applied to correct the pressure term. One of the most common is Semi-Implicit Method for Pressure-Linked Equations (SIMPLE). The algorithm assumes that the flow goes from higher to lower pressure zones (VERSTEEG; MALALASEKERA, 2007). Based on the pressure from the last iteration, Navier-Stokes equations are solved, taking the velocities as a solution. The mass imbalance is evaluated, and in the case of extra mass flowing into, the pressure of this element must be too low when compared to the neighbors. A pressure correction equation is applied in function of mass imbalance. Afterward, turbulence equations are solved, finishing the procedures for one iteration.

The coupled flow solver solves the pressure and velocities simultaneously. The Continuity and Navier-Stokes equations are treated as a vector of equations:

$$
\oint_{\partial \Omega}[\mathbf{F}-\mathbf{G}] \cdot d \mathbf{s}=\int_{\Omega} \mathbf{H} d \Omega
$$




$$
\mathbf{F}=\left[\begin{array}{c}
\rho \mathbf{u} \\
\rho \mathbf{u} \otimes \mathbf{u}_{\mathbf{r}}+p \mathbf{I}
\end{array}\right] \quad \mathbf{G}=\left[\begin{array}{c}
0 \\
\mathbf{T}
\end{array}\right] \quad \mathbf{H}=\left[\begin{array}{c}
0 \\
-\rho \boldsymbol{\omega} \times \mathbf{u}
\end{array}\right]
$$

After getting pressure and velocities, the algorithm solves the turbulence equations. The preconditioned formulation for the governing equations is used to make it suitable for solving incompressible flows.

The segregated solver uses less memory (Siemens, 2017), which is the default method and most used for FVM codes (BAKKER, 2006), and it is used as the primary method for this work. Coupled solver has the advantage of robustness for solving flows with dominant source terms, such as rotation, and is used for optimization problems, once the adjoint module is available with the Coupled solver. 


\section{Topology Optimization Method}

\subsection{Topology Optimization applied to Fluid Flow problems}

Topology Optimization Method has been developed initially for structural problems (BENDSØE; KIKUCHI, 1988), but its application has been growing in the field of fluids. In this method, to obtain the sensitivity of the cost function with relation to design variables is highly important to ensure good convergence in a feasible time during the optimization process. Topology Optimization of fluid flow was first introduced by Borrvall and Petersson (2003). In their pioneering work, they used a finite element approach to study a Stokes flow problem. The objective was to minimize flow dissipated power subjected to a volume constraint, as shown in Fig. 9.

Figure 9 - Fluid Topology Optimization conditions and results.

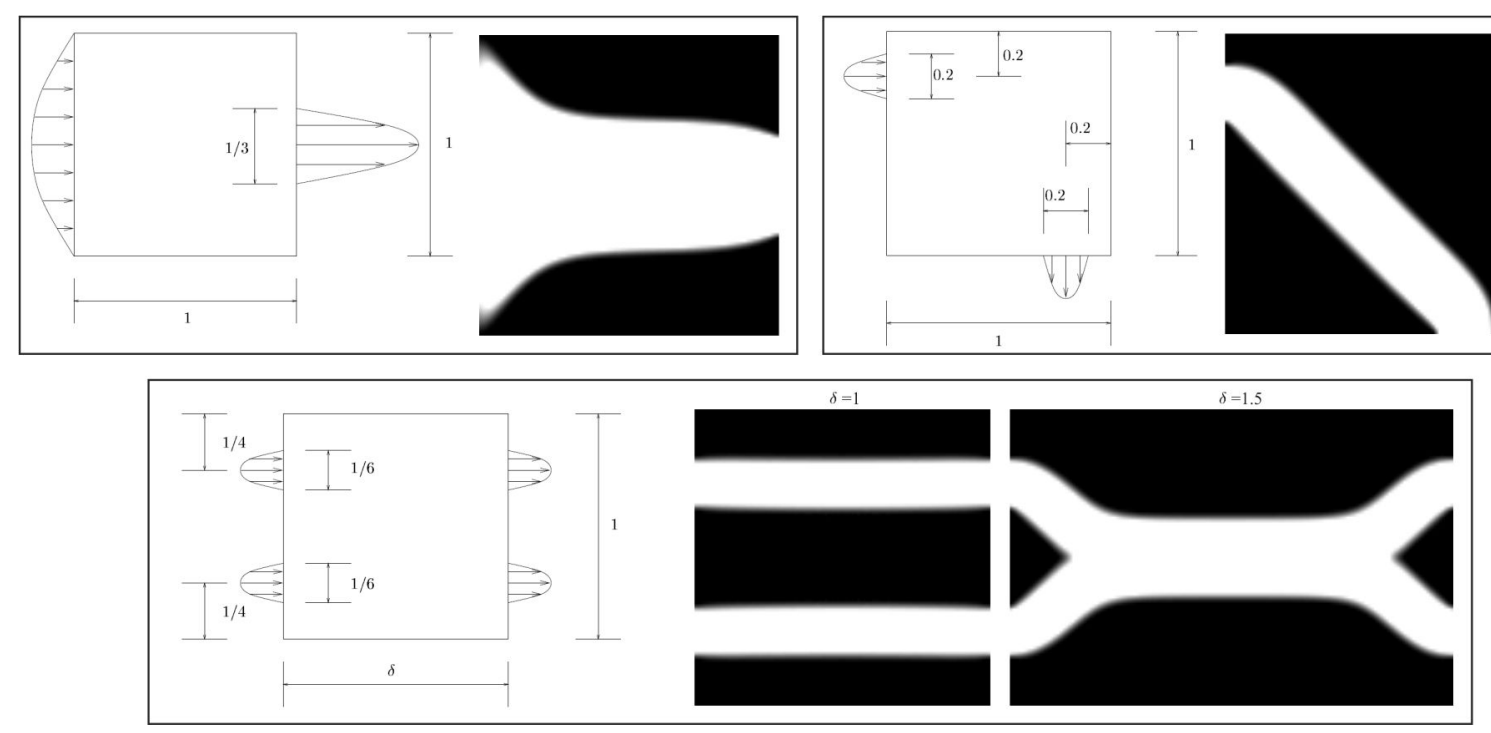

Source: Borrvall and Petersson (2003)

Their work was extended by Evgrafov (2005), who included the limiting cases of pure liquid and solid. Gersborg-Hansen, Sigmund and Haber (2005) extended the applications of TOM to Navier-Stokes flow to cover from low to moderate Reynolds numbers in 2D problems. Guest and Prévost (2006) studied a variation of the method where the Stokes and Darcy equations exist as two different models, which are combined and scaled according to the permeability of each element. Othmer (2008) presented a methodology to apply the TOM for turbulent flows by using FVM.

In other traditional works, the objective is to find a path that minimizes a cost function. The definition of what each element represents, whether it is a solid or a fluid, 
changes with each iteration according to the optimizer. In this work, the optimizer changes the boundary condition that is applied to the solid. The optimizer does not change solid geometry and, thus, wall distance, for example, does not change according to the optimizer.

\subsection{Design procedure}

Topology Optimization formulation consists of the change of design variables to improve a cost function value. The direct problem can be solved numerically by FVM and using an optimizer algorithm. In this work, design variables change the presence or absence of material (in this case, the presence or absence of SH paint) within a domain, which is a surface domain. The design procedure is illustrated in Fig. 10.

Figure 10 - Optimization procedure for surface topology optimization on a propeller's blade

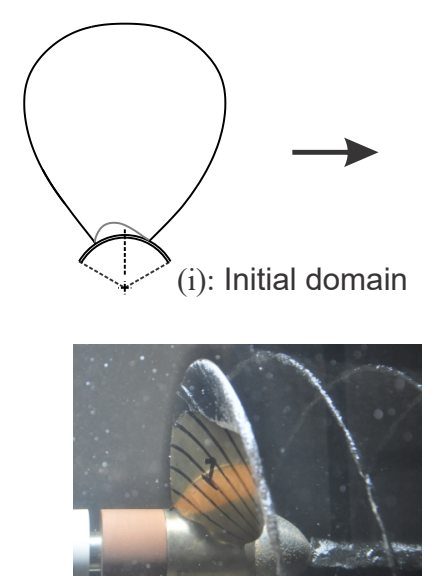

(vi): Test
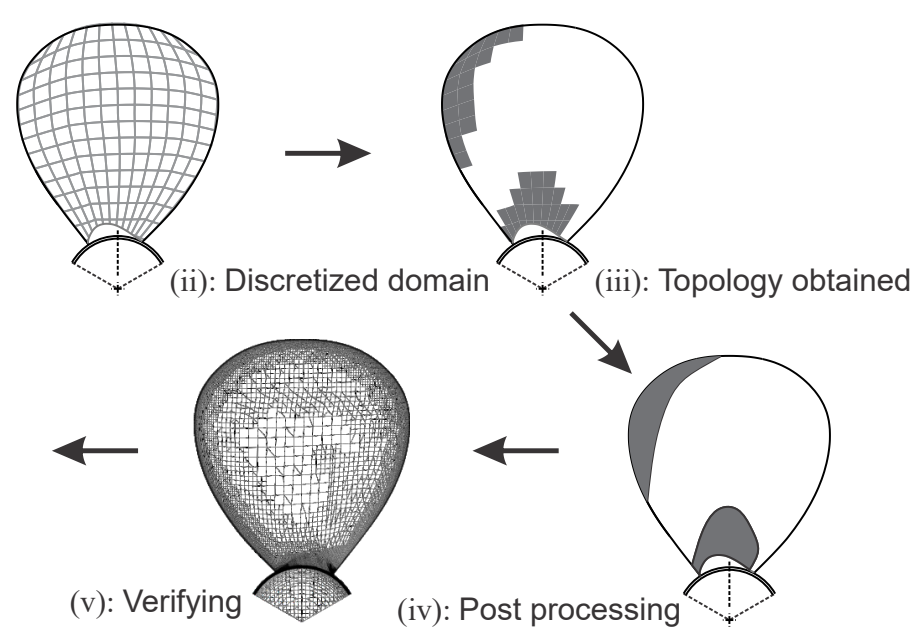

(iv): Post processing

Source: Author

Initially, an initial domain with a controlled material distribution is defined. Then, the domain is discretized and divided the domain into elements. With the generated mesh, nodes and degrees of freedom are defined. In general, design mesh used for optimization differs from the CFD mesh, since using the same amount of elements could unnecessarily increase the computational cost for optimization. Likewise, in this work, a coarse CFD mesh is used for the surface design of paint distribution, being used in the optimization framework, and a finer CFD mesh is reused to verify the results.

With the discretized domain, topology optimization takes place. The flow is solved and the cost function is computed, along with its gradients, being fundamental for gradientbased optimizers to determine the next iteration. The process continues until it reaches pre-defined stop criteria that observe the convergence of the material distribution. The numerical implementation is discussed in detail in Sec. 5. 
After the convergence, post-processing is performed. The result of the optimization is interpreted and reevaluated numerically in a finer CFD mesh. This finer mesh can not be able to be used during the optimization framework due to a high computational cost. This verification step is essential to evaluate if the desired objective is reached even with the post-processed modification.

Finally, the experimental test can be performed. This stage requires the ability to reproduce the same hydrophobic conditions, with pre-established models, which is not the scope of this work.

\subsection{Optimizer}

Numerical algorithms for solving optimization problems are mainly classified in methods of mathematical programming and probabilistic methods. Because it is a problem with state equations in continuous space, the mathematical programming methods are chosen. According to Haftka and Gürdal (1992), although probabilistic methods get a direction to global minimum/maximum, computational cost increases considerably with the number of design variables, when compared to mathematical programming algorithms, deteriorating the solution efficiency, which is critical in topology optimization problems with several variables. Moreover, there is no way to prove that they actually found the global point, which means that there is also the chance of obtaining only local minimums. Finally, many continuous problems, when defined in the discrete space, present several solutions (or no solution) due to the problem of the multiple local minima that arise in the discrete solution space. In other words, the solution oscillates between the different minimums, which is not the case in the continuous case.

\subsection{Sensitivities}

In most optimization algorithms, including what is adopted in this work, it is necessary to calculate the gradients of the cost function, also called sensitivities. The sensitivities are of paramount importance as they guide the optimizer for the optimum solution and, if inaccurately calculated, it would guide to the wrong directions, consuming more time and computational cost or even not getting to an optimized solution. Sensitivity has an advantage in providing information on the system behavior. In some cases, it is possible to analyze the change in the behavior of the system due to a small change of design variables without necessarily performing new analyses, reducing computational costs.

When using methods to obtain the gradient, such as Finite Difference Method (FDM), the number of simulations (cost function evaluations) required for its determination 
increases notably with the number of design variables. This fact contributes to limit the number of design variables for optimization. From the need for a quick way to get the sensitivity, adjoint methods are a good solution. The method determinates gradients similar to that used in CFD by using iterative methods and FVM, for example. The cost function is evaluated by solving the so-called primal problem. Then, equations and boundary conditions are changed, and some results from primal solution are loaded, to solve the so-called adjoint problem.

\subsection{Adjoint method}

As described above, the TOM has a large number of design variables, which are the finite volumes that are discretized in the domain, as previously described. It becomes necessary to obtain an efficient and feasible method of gradient calculation for a large number of design variables. The adjoint methods are virtually independent of the number of design variables, making them ideals for topology optimization.

The adjoint methods have been used since the 50s, with applications that are not limited to fluids, but also in financial operations and computer graphics. One of the first optimization using adjoint methods is performed by Pironneau (1973), in which the objective is to minimize drag in a Stoke flow. Since then, it has been used more frequently as a method to calculate the sensitivity, mainly in the aeronautical industry, such as in Reuther et al. (1999). However, in the automotive industry, development has happened more recently. According to Othmer (2014), this is because the automotive industry relies almost exclusively on commercial CFD software, which does not allow direct access to code, making it challenging to implement the adjoint methods.

For the numerical implementation code to solve the adjoint problem, it can use a continuous or discrete approach, as shown in Fig. 11.

Figure 11 - Continuous and discrete adjoint approach.

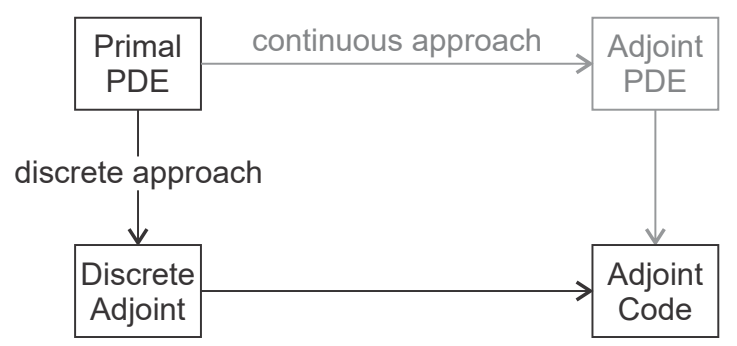

Source: Author

The two approaches start from the PDE for fluids, such as continuity and NavierStokes equations. Continuous approach gets the adjoint equations in the continuous form, resulting in other PDEs. Then, these equations are discretized in the same way and using 
the same mesh as the discrete form of the primal problem, obtaining the adjoint code. In the case of the discrete approach, the primal equations are discretized, linearized, and transposed. The continuous approach has the advantage of low memory consumption and can be implemented in a straightforward form in $\mathrm{C}++$ framework, the same language as Interior Point Optimizer (IPOPT). The advantages of the discrete approach are to obtain the gradient of the discretized cost function, which allows for the optimization process to converge fully. More details on the differences between approaches can be found in Giles and Pierce (2000).

Therefore, the TOM is chosen because of its versatility in finding painting patterns and the adjoint method to obtain its sensitivities. The discrete approach is chosen for the advantages cited above, and its derivation is described in the following chapter.

\subsection{Material model and Surface Constraint}

From a topology optimization point of view, the slip length value is given by a value within the range $[\underline{b} ; \bar{b}]$. It is desired to have a function that relates the minimum and maximum values of $b$ by a range of pseudo-density $\hat{\rho}$ from 0.0 to 1.0. Thus, zero pseudo-density results in $\underline{b}$ and pseudo-density of 1.0 results in $\bar{b}$. In this case, it is desired to have a correlation function that represents a no-slip condition (no-SHS) for the lowest value of $\hat{\rho}$, which implicates in $\underline{b}=0.0$. A function is desired that results in values close to one at the extremes; otherwise, it could result in intermediate slip length values, which can be infeasible when dealing with SHS paint (the only options are painted or not painted regions).

It is required to penalize intermediate values to allow an interpretation: although it is possible to have a surface covered by several slip lengths, it is studied the application of a painting that imposes an SH behavior. This painting is assumed to apply a constant value of slip length. In this sense, it is important to have extremized values of $\hat{\rho}$, which can be interpreted in the real world as to where to apply or not the painting.

Based on the Simplified Isotropic Material with Penalization (SIMP) model (ROZVANY; ZHOU; BIRKER, 1992), the following material model is proposed with a penalization $q$ in the pseudo-density to avoid intermediate values:

$$
b(\hat{\rho})=\underline{b}+(\bar{b}-\underline{b}) \hat{\rho}^{q}
$$

In Fig. 12, it is possible to observe the effect of penalization with respect to (w.r.t.) values of pseudo-densities. This penalization bias intermediate values for some extreme values of $b$.

Another approach extremize values of pseudo-density $\hat{\rho}$ is to restrict the sum of 
Figure 12 - SIMP Model example for several penalization factor $q$ in linear (left) and log-scale (right)

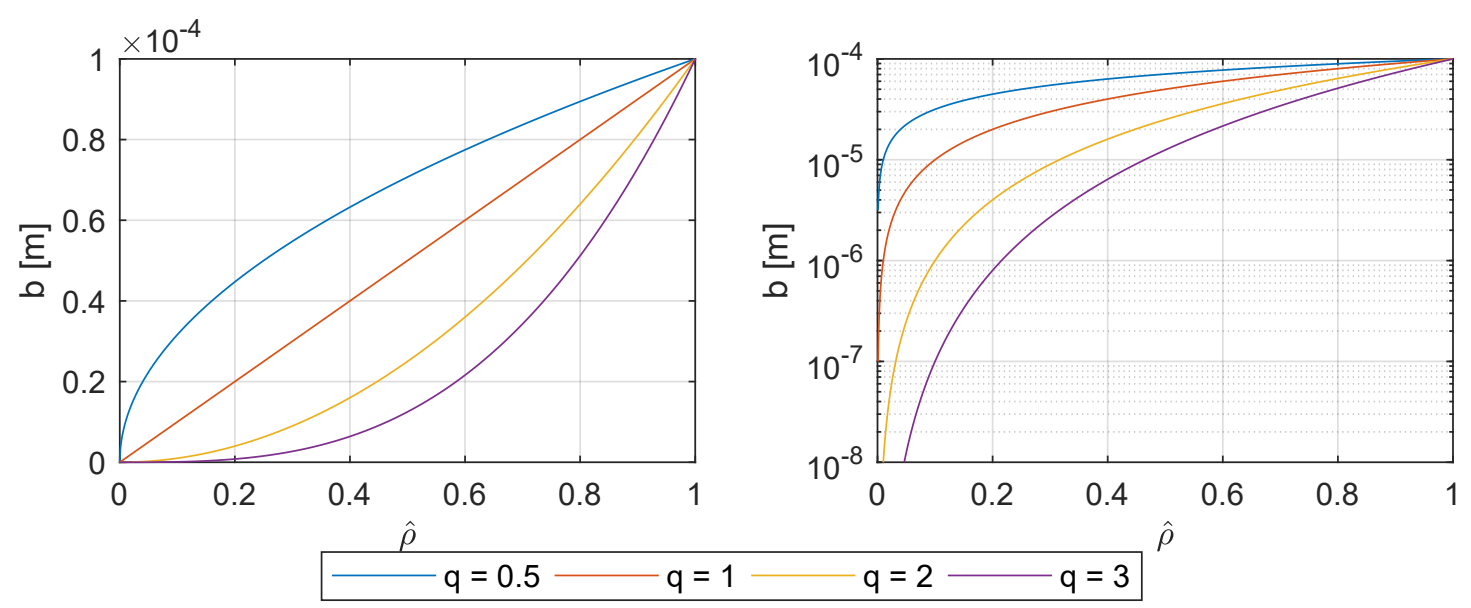

Source: Author

$\hat{\rho}$ in design domain. This procedure is done in several fluid topology optimization, as described in Bendsøe and Kikuchi (1988) and applied in Gersborg-Hansen, Sigmund and Haber (2005), Pingen and Maute (2010), Romero and Silva (2014) or Dilgen et al. (2018). Once pseudo-density is a design variable that ranges between 0.0 and 1.0, it its meaning is also interpreted as the percentage of surface covered by $\mathrm{SH}$ painting.

The formulation for surface-average value of pseudo-density is:

$$
\hat{g}=\frac{\int_{\Theta} \hat{\rho} d s}{\int_{\Theta} d s} \stackrel{\text { discret }}{\Longrightarrow} \frac{\sum_{f} \hat{\rho} A_{f}}{\sum_{f} A_{f}}
$$

in which $\Theta$ is the design surface, and the subindex $f$, discretized faces of $\Theta$.

\subsection{Optimization Formulation}

The TOM is applied in two cases: 2D and 3D cases.

For 2D cases, it is divided in two sub-cases: external and internal flow cases. For external flow, the optimization formulation is defined by Eq. (3.3), in which is desired to reduce drag, once it directly contributes to the energy consumed for movement, with a limited amount of surface fraction $\hat{g}^{*}$ that can be SHS.

$\begin{array}{|ll|}\operatorname{Min}_{\hat{\rho}} & C=c_{d}=\frac{2}{\rho v_{a}^{2} c} D \\ \text { such that (s.t.) } & \hat{g}(\hat{\rho})=\frac{\int_{\Theta} \hat{\rho} d A}{\int_{\Theta} d A} \leq \hat{g}^{*} \\ & 0 \leq \hat{\rho} \leq 1\end{array}$


in which $C$ is the cost function; $D$, drag force (per unit of span); $v_{a}$, advance velocity (in the far-field); and $c$, chord.

For the internal flow case, the optimization formulation is defined by Eq. (3.4), in which it is desired to reduce the pressure drop $p_{\text {drop }}$ between inlet and outlet.

$$
\begin{array}{|ll}
\operatorname{Min}_{\hat{\rho}} & C=p_{\text {drop }}=\frac{\int\left[p+\frac{1}{2} \rho|\mathbf{u}|^{2}\right] d \dot{m}}{\int d \dot{m}} \\
\text { s.t. } & \hat{g}(\hat{\rho})=\frac{\int_{\Theta} \hat{\rho} d A}{\int_{\Theta} d A} \leq \hat{g}^{*} \\
& 0 \leq \hat{\rho} \leq 1
\end{array}
$$

in which $p$ is the pressure; $|\mathbf{u}|$, velocity magnitude; and $\dot{m}$, mass-flow.

For 3D problems, which is the propeller case, the objective is to increase the hydrodynamic efficiency, defined in Eq. (2.4). Therefore, the optimization formulation is given by:

$$
\begin{array}{ll}
\underset{\hat{\rho}}{\operatorname{Max}} & C=\eta \\
\text { s.t. } & \hat{g}(\hat{\rho})=\frac{\int_{\Theta} \hat{\rho} d A}{\int_{\Theta} d A} \leq \hat{g}^{*} \\
& 0 \leq \hat{\rho} \leq 1
\end{array}
$$





\section{Discrete Adjoint Method for Navier-Stokes equations}

The gradients of cost function w.r.t. design variables are required for the optimization algorithm. Since the optimization is an iterative process, the gradients are evaluated several times for several different conditions. Getting these sensitives quickly has a considerable impact on the overall computational time of the optimization process. This chapter describes the sensitivity calculation using discrete adjoint formulation applied to super-hydrophobic modeling. As it will be shown, this chapter describes how to convert a 3D-space adjoint solution to 2D-space sensitivity. In the end, the final expression for sensitivities for 2D and 3D cases cost function are presented.

\subsection{Adjoint method}

Consider a system of PDE given by Eq. (4.1), in which $\mathbf{q}$ is the output of PDE solution (e.g.: for fluid problems, $\mathbf{q}$ can be an array with pressure and velocities that satisfies Navier-Stokes equations) and $m$, design variable.

$$
\mathbf{f}(\mathbf{q}(m), m)=0
$$

Consider a cost function $C(\mathbf{q}(m), m)$ with the same input parameters. By deriving the cost function and by applying the chain rule:

$$
\frac{d C}{d m}=\frac{\partial C}{\partial \mathbf{q}} \frac{d \mathbf{q}}{d m}+\frac{\partial C}{\partial m}
$$

The last term of Eq. (4.2) is easily determined since the cost function expression is well-defined. The difficulty consists in to obtain the implicit part, which is obtained by deriving the system of PDE with the chain rule:

$$
\frac{d \mathbf{f}}{d m}=\frac{\partial \mathbf{f}}{\partial \mathbf{q}} \frac{d \mathbf{q}}{d m}+\frac{\partial \mathbf{f}}{\partial m}=0
$$

Rearranging the terms yields:

$$
\frac{d \mathbf{q}}{d m}=-\left(\frac{\partial \mathbf{f}}{\partial \mathbf{q}}\right)^{-1} \frac{\partial \mathbf{f}}{\partial m}
$$


Inserting the obtained expression into Eq. (4.2), the adjoint vector $\boldsymbol{\lambda}$ is defined:

$$
\frac{d C}{d m}=-\underbrace{\frac{\partial C}{\partial q}\left(\frac{\partial \mathbf{f}}{\partial \mathbf{q}}\right)^{-1}}_{\boldsymbol{\lambda}} \frac{\partial \mathbf{f}}{\partial m}+\frac{\partial C}{\partial m}
$$

Finally, the sensitivity of a cost function $C$ w.r.t. $m$ is:

$$
\frac{d C}{d m}=-\boldsymbol{\lambda} \cdot \frac{\partial \mathbf{f}}{\partial m}+\frac{\partial C}{\partial m}
$$

The adjoint solution vector $\boldsymbol{\lambda}$ is obtained in this work by using the adjoint flow solver included in Star-CCM+. More details on the numerical approach of how to obtain the adjoint vector with feasible computational cost is described in Siemens (2017). The next sections discuss how to obtain each term of the expression above.

\subsection{Derivative of residual with respect to slip length}

Consider the vector $\mathbf{f}$ given by a Continuity, $f_{c}$, and Navier-Stokes residuals, $\mathbf{f}_{\mathbf{m}}$. Therefore:

$$
\mathbf{f}=\left[\begin{array}{l}
f_{c} \\
\mathbf{f}_{\mathbf{m}}
\end{array}\right]
$$

The derivative of residual w.r.t. design variable applied to SHS is not as trivial as determining for classical fluid topology optimization. In classical fluid topology optimization problems, such as Borrvall and Petersson (2003), the objective is to determine a path that minimizes the energy dissipation. Therefore, the Brinkman model is used to penalize the fluid around some regions and simulate a wall condition.

In a nutshell, a source term is added in Navier-Stokes equations that are lineardependent between the design variable and velocity (Darcy's Law), Eq. (4.8). For $m$ larger enough to penalize the velocity around that region, the solid condition is simulated.

$$
\mathbf{f}_{\mathbf{m}}=\rho(\mathbf{u} \cdot \nabla) \mathbf{u}+\nabla p-\nabla \cdot(2 \mu \mathbf{D})-m \mathbf{u}
$$

In fluid topology optimization problems with the Brinkman model, derivative of residual w.r.t. design variable is easily defined as:

$$
\frac{\partial \mathbf{f}_{\mathbf{m}}}{\partial m}=-\mathbf{u}
$$

To test the numerical implementation, the Brinkman model is implemented and described in Appendix A. 
However, for SHS problems, the slip length $b$ is not explicitly written as for the Brinkman term. The approach to determine this derivative is to consider the discrete form. It is assumed that all elements that do not contact an SHS (design surface) have zero influence on the sensitivity. Thus, only near-wall elements of the design surface are analyzed.

Consider a representation of mesh element that contacts with a design surface, as shown in Fig. 13. This element, with volume $\Omega$, is formed with two surface groups: $\Theta$, in full contact with a design surface (in which can be an SHS); and $\Gamma$, the remaining surfaces, that are in contact with other elements. The sum of these two surface groups is the surface of the volume, $\partial \Omega=\Gamma \cup \Theta$.

Figure 13 - Near-wall element schema and its surfaces

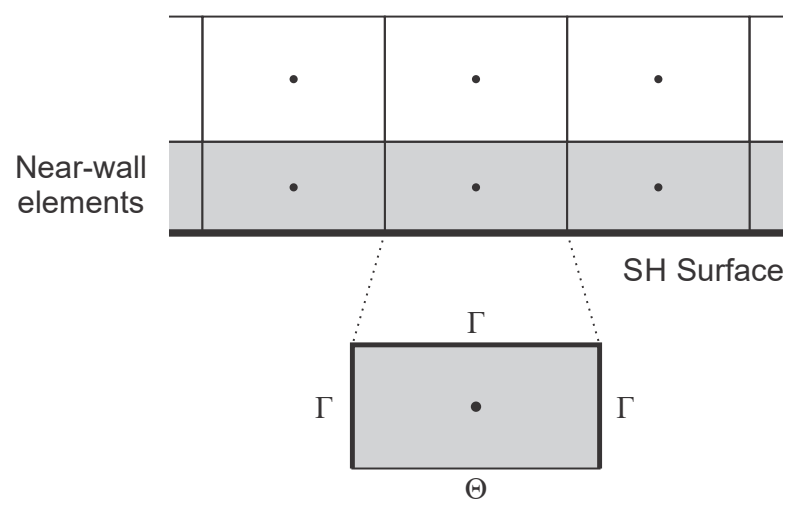

Source: Author

By applying the Continuity and Navier-Stokes equations in this near-wall elements yields:

$$
\begin{gathered}
f_{c}=\int_{\Gamma} \mathbf{u} \cdot d \mathbf{s}+\int_{\Theta} \mathbf{u} \cdot d \mathbf{s} \\
\mathbf{f}_{\mathbf{m}}=\int_{\Gamma}(\rho \mathbf{u} \otimes \mathbf{u}) \cdot d \mathbf{s}+\int_{\Gamma} p \mathbf{I} \cdot d \mathbf{s}-\int_{\Gamma} 2\left(\mu+\mu_{t}\right) \mathbf{D} \cdot d \mathbf{s}+ \\
\int_{\Theta}(\rho \mathbf{u} \otimes \mathbf{u}) \cdot d \mathbf{s}+\int_{\Theta} p \mathbf{I} \cdot d \mathbf{s}-\int_{\Theta} 2\left(\mu+\mu_{t}\right) \mathbf{D} \cdot d \mathbf{s}
\end{gathered}
$$

Note that only $\Theta$ surface has a dependency of $b$ in the formulation. Also, even though it is an SHS, the impermeability condition is maintained. So, the convective term in $\Theta$ is zero. Thus, Continuity and Navier-Stokes equations are reduced to:

$$
\begin{gathered}
f_{c}=\int_{\Gamma} \mathbf{u} \cdot d \mathbf{s} \\
\mathbf{f}_{\mathbf{m}}=\int_{\Gamma}(\rho \mathbf{u} \otimes \mathbf{u}) \cdot d \mathbf{s}+\int_{\Gamma} p \mathbf{I} \cdot d \mathbf{s}+\int_{\Theta} p \mathbf{I} \cdot d \mathbf{s}-\int_{\Gamma} 2\left(\mu+\mu_{t}\right) \mathbf{D} \cdot d \mathbf{s}-\int_{\Theta} 2\left(\mu+\mu_{t}\right) \mathbf{D} \cdot d \mathbf{s}
\end{gathered}
$$


Close to the wall, the turbulent kinetic energy is close to zero, and consequently, the turbulent viscosity ratio is also close to zero, allowing the approximation $\mu+\mu_{t} \approx \mu$. Also, to emphasize only terms that contain $b$ in the formulation:

$$
\begin{gathered}
f_{c}=[\ldots] \\
\mathbf{f}_{\mathbf{m}}=-\int_{\Theta} 2 \mu \mathbf{D} \cdot d \mathbf{s}+[\ldots]
\end{gathered}
$$

In other words, $f_{c}$ has no explicit dependency of $b$. It implies that only $\mathbf{f}_{\mathbf{m}}$ influences the implicit part of sensitivity calculation.

According to (Siemens, 2017), the gradient of velocity on the surface is numerically calculated as:

$$
\nabla \mathbf{u} \cdot \mathbf{s}=\left[\frac{\mathbf{u}_{\mathbf{c}}-\mathbf{u}_{\mathbf{f}}+\left(\mathbf{u}_{\mathbf{f}} \cdot \mathbf{n}\right) \mathbf{n}}{\mathbf{d} \cdot \mathbf{n}}\right]|\mathbf{s}|
$$

in which $\mathbf{u}_{\mathbf{c}}$ is the velocity at cell center; $\mathbf{d}$, distance vector between the face and cell center; and $\mathbf{u}_{\mathbf{f}}$, velocity on the face (on SHS, $\mathbf{u}_{\mathbf{f}}$ is given by Eq. (2.34)). Figure 14 illustrates each variable in a near-wall element.

Figure 14 - Schema of velocities in the near-wall element

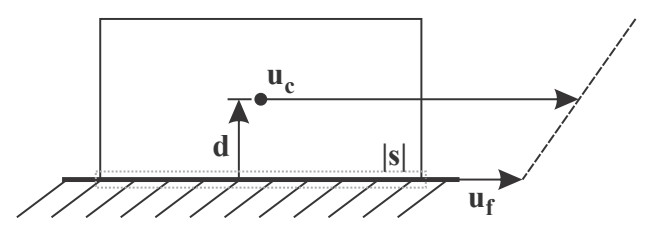

Source: Author

Since the impermeability condition is still satisfied, Eq. (4.16) is reduced to:

$$
\frac{\partial \mathbf{u}}{\partial \mathbf{n}} \cdot \mathbf{s} \stackrel{\text { discret }}{\Longrightarrow} \frac{\mathbf{u}_{\mathbf{c}}-\mathbf{u}_{\mathbf{f}}}{\mathbf{d} \cdot \mathbf{n}}|\mathbf{s}|
$$

By assuming a low wall $y^{+}$approach to model the near-wall elements, it is expected that these elements are simulating the viscous sub-layer, in which there is a linear relationship between velocity and wall distance. Even though, for laminar flows it is possible to use Eq. (4.16) to estimate the gradient. In this way, the following expression can also be applied to laminar flow simulations.

By applying the numerical expression for the gradient to calculate the surface integral of the shear stress component on $\Theta$ :

$$
\int_{\Theta} 2 \mu \mathbf{D} \cdot d \mathbf{s}=\int_{\Theta} \mu \frac{\partial \mathbf{u}}{\partial \mathbf{n}} d A \stackrel{\text { discret }}{\Longrightarrow} \mu \frac{\mathbf{u}_{\mathbf{c}}-\mathbf{u}_{\mathbf{f o}}(b)}{d} A
$$


in which $d=|\mathbf{d} \cdot \mathbf{n}|$ and $A=|\mathbf{s}|$.

Since water is a Newtonian fluid, it holds the following relation between shear stress and strain rate.

$$
\boldsymbol{\tau}=\left.\mu \frac{\partial \mathbf{u}}{\partial \mathbf{n}}\right|_{\Theta}
$$

Thus, the velocity on the face, given by slip length expression in Eq. (2.34), can be written by observing the shear stress expression for Newtonian fluids.

$$
\mathbf{u}_{\mathbf{f}}=\left.b \frac{\partial \mathbf{u}}{\partial \mathbf{n}}\right|_{\Theta}=b \frac{\boldsymbol{\tau}}{\mu}
$$

Equation (4.18) can be rewritten to consider the SHS modeling:

$$
\int_{\Theta} 2 \mu \mathbf{D} \cdot d \mathbf{s} \stackrel{\text { discret }}{\Longrightarrow} \frac{\mu A}{d}\left[\mathbf{u}_{\mathbf{c}}-b \frac{\boldsymbol{\tau}}{\mu}\right]
$$

Finally, by inserting Eq. (4.21) in Eq. (4.15) yields:

$$
\mathbf{f}_{\mathbf{m}}=-\frac{\mu A}{d}\left[\mathbf{u}_{\mathbf{c}}-b \frac{\boldsymbol{\tau}}{\mu}\right]+[\ldots]
$$

The formulation of $\mathbf{f}_{\mathbf{m}}$ is now written such that $b$ is explicitly in the formulation. By deriving and remembering that terms on $\Gamma$ have null derivative w.r.t. $b$ :

$$
\frac{\partial \mathbf{f}_{\mathbf{m}}}{\partial b}=\frac{A}{d} \boldsymbol{\tau}+{ }_{\alpha} \mathcal{r}_{\Gamma}^{0}
$$

or assembling into Eq. (4.7) format:

$$
\frac{\partial \mathbf{f}}{\partial b}=\left[\begin{array}{c}
0 \\
\frac{A}{d} \boldsymbol{\tau}
\end{array}\right]
$$

However, $b$ is not exactly a design variable: as discussed in Sec. $3, b$ is implemented through a pseudo-density using SIMP model, described by Eq. (3.1). By deriving $b$ w.r.t. pseudo-density $\hat{\rho}$ yields:

$$
\frac{\partial b}{\partial \hat{\rho}}=\bar{b} q \hat{\rho}^{q-1}
$$


To obtain the partial derivative of $\mathbf{f}$ w.r.t. design variable $\hat{\rho}$, the chain rule is applied:

$$
\frac{\partial \mathbf{f}}{\partial m}=\frac{\partial \mathbf{f}}{\partial \hat{\rho}}=\frac{\partial \mathbf{f}}{\partial b} \frac{\partial b}{\partial \hat{\rho}}
$$

\subsection{Numerical Checking of Derivatives}

To evaluate the correspondence of the derivative of momentum equation w.r.t. slip length $b$, two simple numerical tests are performed, in order to numerically evaluate the expression. The test case is a flat plate with SHS on it, where a single near-wall element is analyzed.

The first test aims to check Eq. (4.22): linear dependency between $b$ and $\mathbf{f}_{\mathbf{m}}$. For simplicity, it is just analyzed the $x$ component of momentum residual, $f_{m, x}$. The simulation is set-up with $b=1 \times 10^{-4} \mathrm{~m}$. After run and reach the convergence criteria, it should result in a very small value of $f_{m, x}$. Then, the value of $b$ is incremented by $\Delta b$, and all under-relaxation factors are set up to 1 . A single step iteration is run, and the residual is analyzed. This procedure is performed for six $\Delta b$, and results are shown in Fig. 15 (left). As it can be noted, there is a linear dependency between $f_{m, x}$ and $b$, confirming the expression showed before. The slope of this line represents the $x$-component of Eq. (4.23).

Figure 15 - Numerical tests to verify the linearity of Eq. (4.22) and Eq. (4.23)
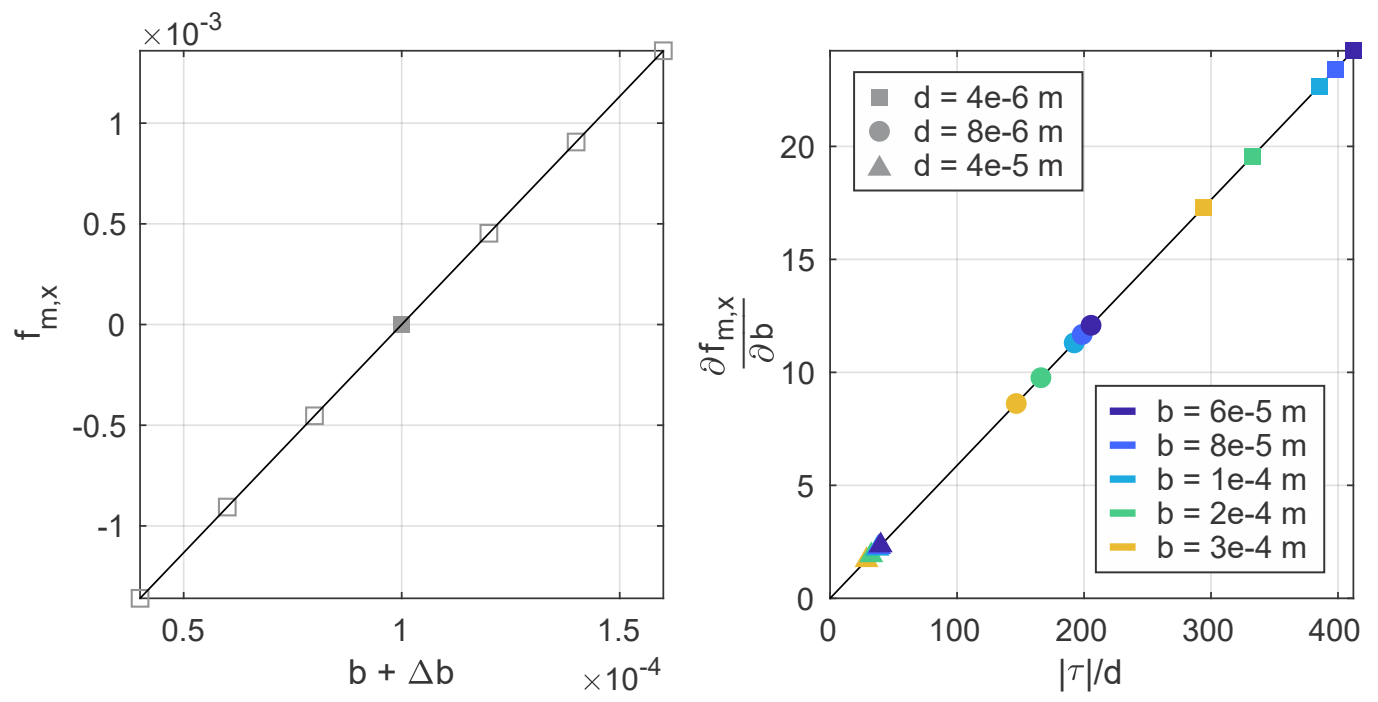

Source: Author

The second test aims to check Eq. (4.23): linear dependency between $\boldsymbol{\tau} / d$ and $\partial \mathbf{f}_{\mathbf{m}} / \partial b$. This line should also cross the origin $(0,0)$. To perform the test, it is considering only the $x$-axis. Five different slip lengths are chosen (which also changes the shear stress) and combined with three different meshes, with different near-wall element sizes. The 
terms $\partial \mathbf{f}_{\mathbf{m}} / \partial b$ are calculated in each condition as the first test: performing a single step iteration with a variation of $b$. Figure 15 (right) confirms the hypothesis: even varying the near-wall thickness and $b$, the regression line with all combination of conditions results in a line crossing the origin, as stated in Eq. (4.23).

\subsection{Partial derivative of drag coefficient with respect to slip length}

For two dimensional cases, minimization of drag coefficient is considered as a cost function, given by:

$$
C=k_{s} c d=k_{s} \frac{2}{\rho v_{a}^{2} c} D
$$

in which $D$ is drag force (per unit of span); $c$, chord; and $k_{s}$, scaling factor (to scale the cost function if the value is numerically too small).

Numerically, drag is calculated as a sum of pressure and shear stress contribution in the advance velocity direction, given as a unit vector $\mathbf{e}_{\mathbf{a}}$ :

$$
D=\sum_{i}^{\Theta}\left[\left(p_{i} \mathbf{I}-\mathbf{T}_{i}\right) \cdot \mathbf{s}_{i}\right] \cdot \mathbf{e}_{\mathbf{a}}
$$

In this expression, the subscript $i$ is designated to each element of design surface.

Deriving the drag expression w.r.t. slip length has a similar procedure to obtain Eq. (4.23). It results in:

$$
\frac{\partial D}{\partial b_{i}}=-\frac{A_{i}}{d_{i}} \boldsymbol{\tau}_{i} \cdot \mathbf{e}_{\mathbf{a}}
$$

Thus, the partial derivative of cost function w.r.t. slip length is:

$$
\frac{\partial C}{\partial b_{i}}=-k_{s} \frac{2}{\rho v_{a}^{2} \bar{c}} \frac{A_{i}}{d_{i}} \boldsymbol{\tau}_{i} \cdot \mathbf{e}_{\mathbf{a}}
$$

\subsection{Partial derivative of pressure drop with respect to slip length}

The pressure drop is given by:

$$
p_{\text {drop }}=\frac{\int_{\text {inletUoutlet }}\left[p+\frac{1}{2} \rho|\mathbf{u}|^{2}\right] d \dot{m}}{\int d \dot{m}}
$$

As can be noted, the pressure drop performs calculations on the surface that is not the design surface. One of the first assumptions made is to consider that all elements that 
do not directly contact with a design surface have zero dependencies on $b$. Therefore, the partial derivative of the pressure drop w.r.t. slip length is zero.

$$
\frac{\partial C}{\partial b_{i}}=\frac{\partial p_{\mathrm{drop}}}{\partial b_{i}}=0
$$

However, it is important to point out that it is possible to convert the pressure drop problem to total potential energy. Sato et al. (2018) deduces the relationship between total pressure drop and total potential energy. In this way, it would be possible to obtain an explicit term of $b$ in the cost function. However, for simplification in this work, this relation is assumed to be zero.

\subsection{Partial derivative of propeller efficiency with respect to slip length}

For the propeller case (three dimensional), the cost function is the maximization of propeller efficiency $\eta$. Since the optimizer works with minimization problems, the cost function is multiplied by -1 .

$$
C=-k_{s} \eta=-k_{s} \frac{T v_{a}}{2 \pi n Q}
$$

In Eq. (4.33), only $T$ and $Q$ are dependent of $b$. Other variables are assumed as constants. Numerically, $T$ and $Q$ are given by:

$$
\begin{gathered}
T=\sum_{i}^{\Theta}\left[\left(p_{i} \mathbf{I}-\mathbf{T}_{i}\right) \cdot \mathbf{s}_{i}\right] \cdot \mathbf{e}_{\mathbf{a}} \\
Q=\sum_{i}^{\Theta}\left[\mathbf{r}_{i} \times\left(p_{i} \mathbf{I}-\mathbf{T}_{i}\right) \cdot \mathbf{s}_{i}\right] \cdot \mathbf{e}_{\mathbf{a}}
\end{gathered}
$$

in which $\mathbf{r}$ is the distance vector between the propeller axis and the current location; and $\mathbf{e}_{\mathbf{a}}$ is unit vector aligned with advance velocity (and propeller axis).

Again, only the shear stress tensors have $b$ dependency. Thus, calculating its derivatives:

$$
\begin{gathered}
\frac{\partial T}{\partial b_{i}}=-\frac{A_{i}}{d_{i}} \boldsymbol{\tau}_{i} \cdot \mathbf{e}_{\mathbf{a}} \\
\frac{\partial Q}{\partial b_{i}}=\left(\mathbf{r}_{i} \times-\frac{A_{i}}{d_{i}} \boldsymbol{\tau}_{i}\right) \cdot \mathbf{e}_{\mathbf{a}}
\end{gathered}
$$


Thus, the derivative of cost function for propeller case w.r.t. slip length is:

$$
\frac{\partial C}{\partial b_{i}}=-k_{s} \frac{v_{a}}{2 \pi n Q^{2}}\left(Q \frac{\partial T}{\partial b_{i}}-T \frac{\partial Q}{\partial b_{i}}\right)
$$

\subsection{General Expression}

In summary, the sensitivity of a cost function $C$ w.r.t. pseudo-density $\hat{\rho}$ associated to an SHS model is given by:

$$
\frac{d C}{d \hat{\rho}_{i}}=-\boldsymbol{\lambda} \cdot \frac{\partial \mathbf{f}}{\partial b_{i}} \frac{\partial b_{i}}{\partial \hat{\rho}_{i}}+\frac{\partial C}{\partial b_{i}} \frac{\partial b_{i}}{\partial \hat{\rho}_{i}}
$$

$\boldsymbol{\lambda}$ is defined in Eq. (4.5), but it is given by the adjoint solver.

$\partial \mathbf{f} / \partial b$ is defined by Eq. (4.24).

$\partial b / \partial \hat{\rho}$ is defined by Eq. (4.25).

$\partial C / \partial b$ depends on the study case. When dealing with external flow case, Eq. (4.30)

is used, for internal flow case, Eq. (4.32), and for propeller case, Eq. (4.38) is used. 



\section{Numerical Implementation}

\subsection{Optimization Flowchart}

The optimization flowchart is schematically described in Fig. 16.

Figure 16 - Topology Optimization Flowchart

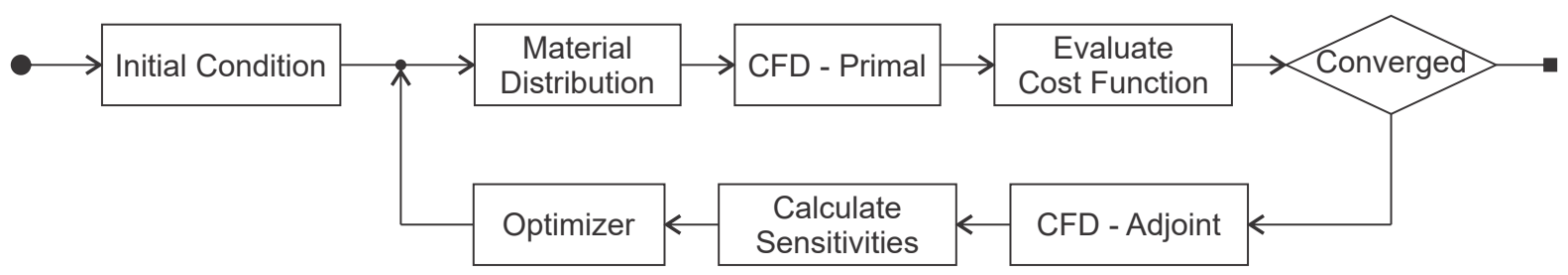

Source: Author

The steps to run the optimization require first to define an initial material distribution. Then, the primal problem is solved to get the hydrodynamic performance with the hydrophobic distribution.

Once the primal problem is solved, the cost function and other properties are evaluated. If the stopping criteria of optimizer are not achieved, it is moved to the adjoint CFD simulation by using the same mesh as the primal problem. The primal solution is frozen, and the adjoint problem is performed.

With the sensitivity information, the optimizer calculates the next design distribution of hydrophobic material. The process is repeated until it reaches the convergence criteria.

\subsection{Primal and Adjoint Solver}

It is desired to choose a software environment that could integrate primal and adjoint solvers without the need for external communications, avoiding extensive data exchange from a typically large number of elements from CFD simulation. It is chosen Siemens' Star-CCM+ 13.06 with double-precision. As detailed in Siemens (2017), it is a cell-centered FVM solver using unstructured grid-solver. The front-end is based on Java, while back-end, in $\mathrm{C}++$ language. One of the advantages is the integration of the pre-processing, solver, and post-processing in the same environment. Star-CCM+ also includes an adjoint module if the primal problem is solved in the Coupled-flow approach. 
Although it is chosen Star-CCM+ as the Primal / Adjoint solver for fluid problems, the problem can be adapted without further modifications to any software, especially if it has an adjoint module.

\subsection{Software Optimizer}

In this work, the IPOPT package (WÄCHTER; BIEGLER, 2006) is used, which uses the Internal Point Optimization algorithm. The IPOPT software applies a primaldual barrier method that solves a sequence of barrier problems. The algorithm is briefly described as follows, but the complete procedure and mathematical background can be found in its reference.

A generic minimization problem with constraints is given by:

$$
\begin{array}{ll}
\underset{\mathbf{x}}{\operatorname{Min}} & C=f(\mathbf{x}) \\
\text { s.t. } & \mathbf{c}(\mathbf{x})=0 \\
& \mathbf{x} \geq 0
\end{array}
$$

The Barrier Function converts bounded problems by a natural logarithm barrier for inequality constraints. As the log term is undefined for negative values, this procedure searches only in feasible interior space. The new objective function is given by:

$$
\begin{array}{ll}
\operatorname{Min}_{\mathbf{x}} & \varphi(\mathbf{x}):=f(\mathbf{x})-\tilde{\mu} \sum_{i=0}^{n} \ln \mathbf{x} \\
\text { s.t. } & \mathbf{c}(\mathbf{x})=0
\end{array}
$$

Observe that the smaller the barrier function $\tilde{\mu}$, the closer the problem is to the original minimization formulation. Since the barrier problem is still a non-linear problem, the Karush-Kuhn-Tucker (KKT) conditions are solved:

$$
\begin{aligned}
\nabla f(\mathbf{x})+\nabla \mathbf{c}(\mathbf{x}) \cdot \tilde{\boldsymbol{\lambda}}-\mathbf{z} & =0 \\
\mathbf{c}(\mathbf{x}) & =0 \\
\mathbf{X Z \mathbf { e }}-\tilde{\mu} \mathbf{e} & =0
\end{aligned}
$$

in which $\tilde{\boldsymbol{\lambda}}$ correspond to the Lagranian multipliers for the equality constraints; $z_{i}=\tilde{\mu} / x_{i}$; $\mathbf{X}$ and $\mathbf{Z}$, matrices with diagonal-only elements filled with values of $\mathbf{x}$ and $\mathbf{z}$, respectively; and $\mathbf{e}$, columns of ones.

This process is repeated iteratively and tries to decrease the $\tilde{\mu}$ until it reaches the convergence criteria. 
In this work, a constraint is given by a function such as Eq. (5.4): limiting the quantity of painting along a surface, expressed by $\hat{g}(\mathbf{x})$, up to a defined quantity $\hat{g}^{*}$.

$$
\hat{g}(\mathbf{x}) \leq \hat{g}^{*}
$$

This type of constraint can be converted to be used in the general format of Eq. (5.1) by using slack variable $s$. For this, the inequality of Eq. (5.4) is converted to an equality and extra inequality constraints in a format similar to the generic minimization problem is added:

$$
\begin{array}{r}
-\hat{g}(\mathbf{x})+\hat{g}^{*}-s=0 \\
s \geq 0
\end{array}
$$

Within this format, the first equation is inside $\mathbf{c}$ vector, while the slack variable is one of the elements inside $\mathbf{x}$.

The implementation strategy used in this work is based on the Nested Analysis and Design (NAND) formulation, in which state variables are not considered for optimization formulation point-of-view. Therefore, governing equations, Continuity and Navier-Stokes, are not inserted into c array.

\subsection{Framework}

Figure 17 shows how Primal/Adjoint solvers and the optimizer communicate with each other.

Figure 17 - Framework of communication between Primal/Adjoint Solver and Optimizer

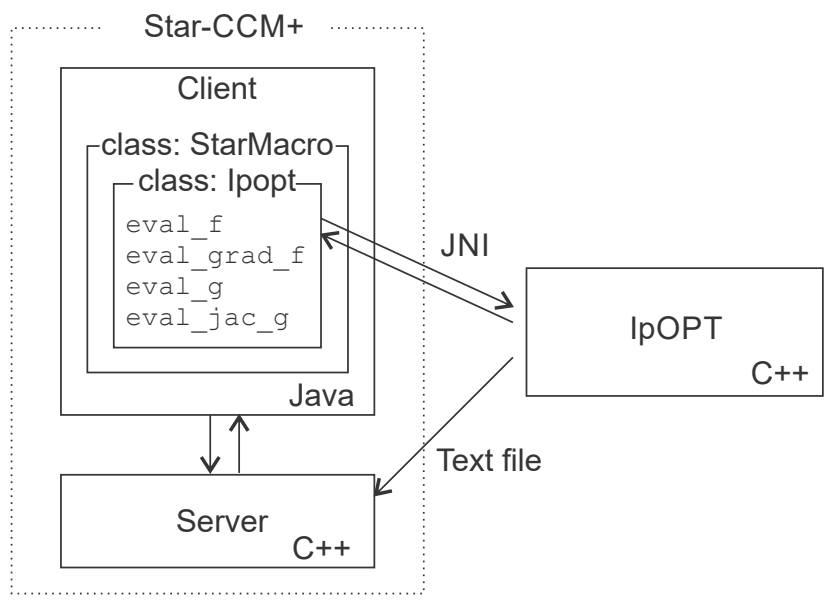

Source: Author

Although the interaction with Star-CCM+ is made in Java language using macros, the server is written in $\mathrm{C}++$ language. The whole framework is inside a Java macro in 
Star-CCM+. Inside this Java macro, it is invoked a Star-CCM+'s class (Star- Macro), and, inside this class, it is also invoked the IPOPT communicator (Ipopt). The Ipopt class communicates through IPOPT using Java Native Interface (JNI), once IPOPT is originally compiled in $\mathrm{C}++$ language. Since the class Ipopt is inside the class StarMacro, it is possible to access the functionalities of Star-CCM+ macro to get results from CFD results, such as values from reports that computes the cost function.

When creating a class Ipopt, some optimization conditions are defined, such as initial condition, stopping criteria, and others. There are some functions inside Ipopt that communicate with IPOPT at each optimization iteration. eval_f is the evaluation of cost function: inside this function, the new painting distribution is applied to the CFD problem and simulate until satisfying the primal problem convergence criteria. Then, the report evaluates the cost function, and the calculated value is returned in the eval_f. For eval_grad_f, the adjoint problem is run until the convergence criteria to obtain the adjoint vector. Then, the report computes the sensitivity and the value is returned. eval_g is the evaluation of constraints and eval_grad_g, its gradients. Since it is just the sum of pseudo-density multiplied by area, it can be done in the Java environment.

The returned values of Ipopt functions are sent to IPOPT through JNI. To receive the painting distribution for the next iteration, a text file, with position and pseudo-density, is created and read in Star-CCM+. Usually, reading and writing file operations can be inefficient in terms of time spend. However, for a single optimization iteration (which includes writing a file from IPOPT, reading a file, solving the primal problem, solving the adjoint problem and output to IPOPT) the comparative time spent on writing/reading files is almost zero. Thus, writing a file as a solution to IPOPT-CFD communication is maintained in the framework.

Since distributing SH painting is a novel application for Fluid Topology Optimization, to test the framework and check its funcionality, it was chosen to simulate a classic fluid topology optimization to ensure that the framework is working as expected. The details presented in Appendix A show that the framework is properly working. 


\section{Methods and results for propeller CFD sim- ulations}

This section, published in Katsuno and Dantas (2016), presents procedures for open-water propeller simulation using CFD for several conditions of advance velocity and rotation. Domain size, mesh topology, refinement level, and turbulence models are analyzed, considering the computational cost, and results are compared with experimental values. The objective of this chapter is to develop a reliable CFD numerical method to be used with accuracy in the cost functions of topology optimization. Since it is not possible to validate the hydrophobic model with experiments, it is desirable to compare the results without a hydrophobic model with physical experiments and apply the same methodology when the SH condition is simulated.

\subsection{Propeller Geometry}

The study is carried out with a Gawn-Burril (KCA) systematic series propeller called H1. Pictures of $\mathrm{H} 1$ are shown in Fig. 18 and propeller design, in Fig. 19. It has three blades, diameter of $0.2 \mathrm{~m}$, pitch-to-diameter ratio of 0.86, EAR (Expanded Area Ratio) of 0.50 , and axis diameter of $0.038 \mathrm{~m}$. Hub and axis are represented in all simulations to get higher similarity with experimental tests at Cavitation Tunnel. Note that the propeller has a rounded tip, smoothing out the propeller edges.

Figure 18 - CAD representation (left); photo of real H1 (center); and inside of cavitation tunnel (right)
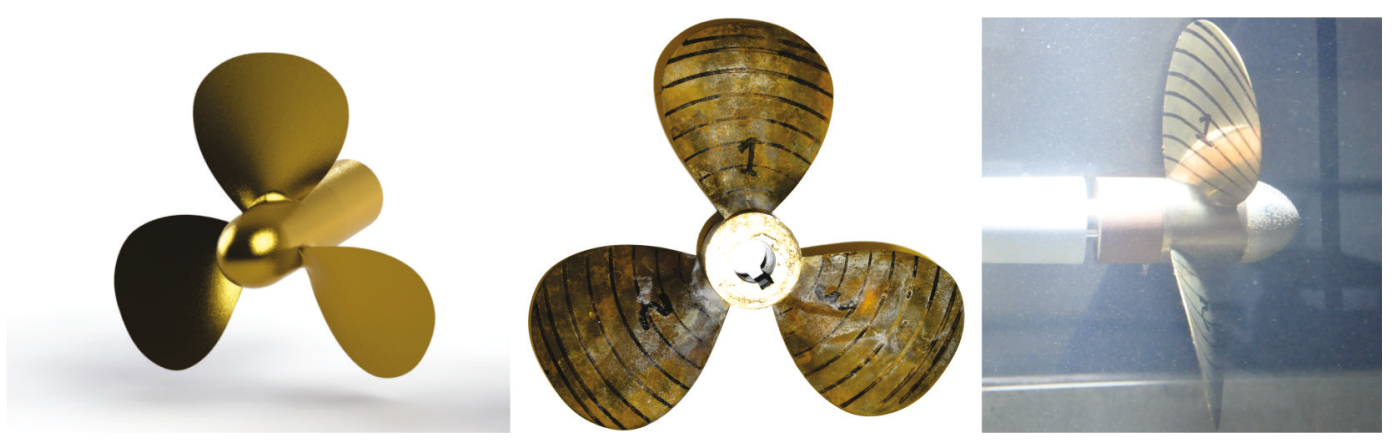

Source: Author 
Figure 19 - Profile, transverse and expanded view of H1 propeller

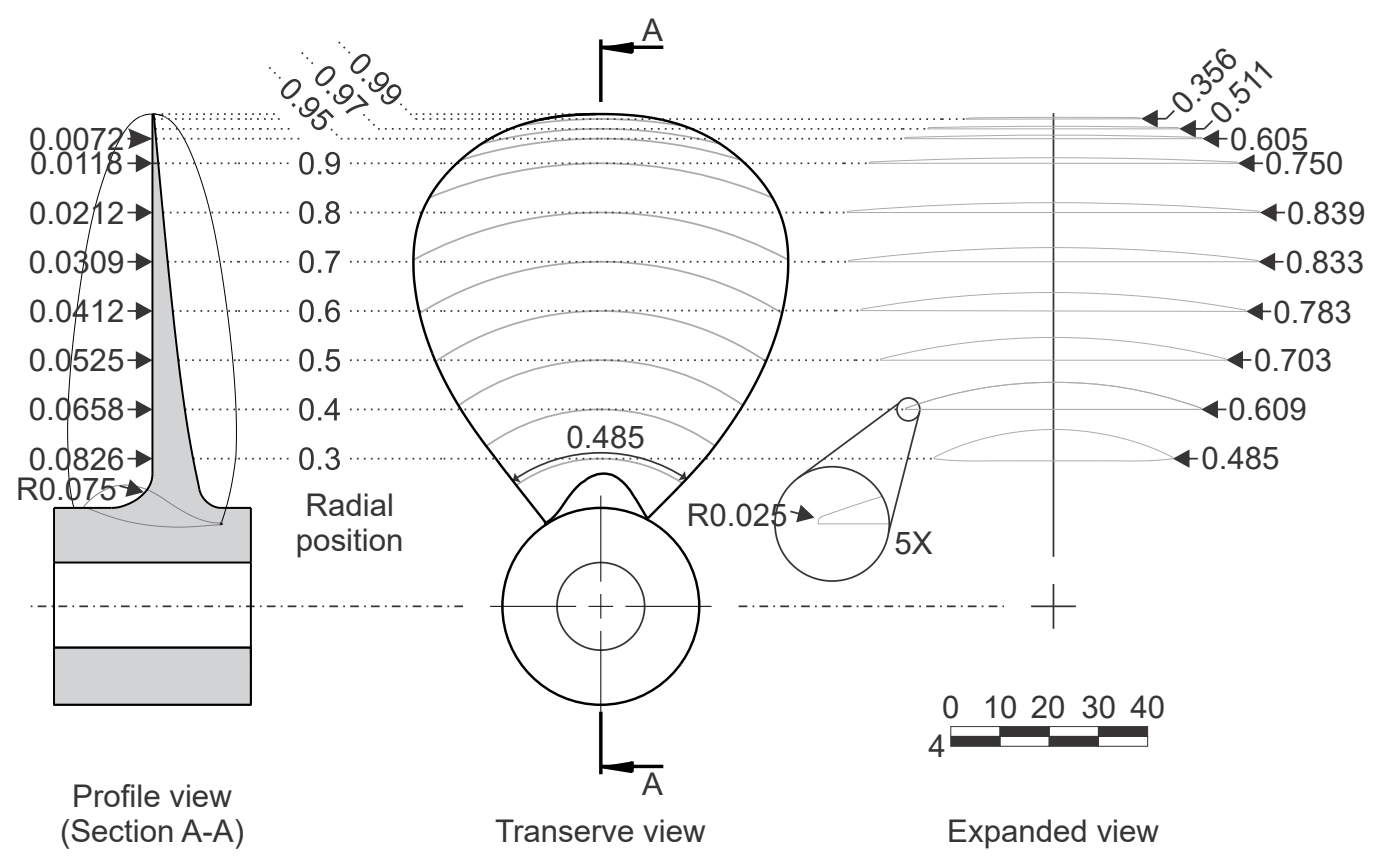

Source: Author

\subsection{Domain Geometry and Boundary Conditions}

The domain is a one-third cylinder, as the propeller has three blades. This simplification is acceptable once it uses RANS equations, which only models large eddies, and the main interest is at the mean values. This simplification was used in other similar works, such as Wang et al. (2010).

The MRF (also called quasi-steady method) is used inside the domain to simulate the rotating effect, and a fixed reference frame is used to define the velocity inlet. More details are described in Sec. 2.3. Sánchez-Caja, Sipilä and Pylkkänen (2009) compared time-accurate propeller simulations with MRF and mixing-plane approach and concluded that MRF approach presented better prediction than the mixing-plane.

To define the domain dimensions in order to minimize the interference effect of cylinder boundaries in the propeller flow, a domain size convergence study is performed by varying two basic dimensions of the cylinder. It is stipulated that downstream dimension (called $d_{x}$, one of the varied values) is three times higher than the upstream dimension. The other variable is the cylinder radius $d_{z}$. Figure 20 shows these two variables, $d_{x}$ and $d_{z}$, both in function of propeller radius $R_{p}$.

Five different values of each variable are simulated with each other, generating a combination of 25 different domain sizes. Both variables have the same five value options: 1.5, 2, 3, 5, and 8. Boundary conditions are presented in Fig. 21, in which the numerical treatments are described in more detail in Sec. 2.5. 
Figure 20 - Domain dimensions evaluated for propeller simulations
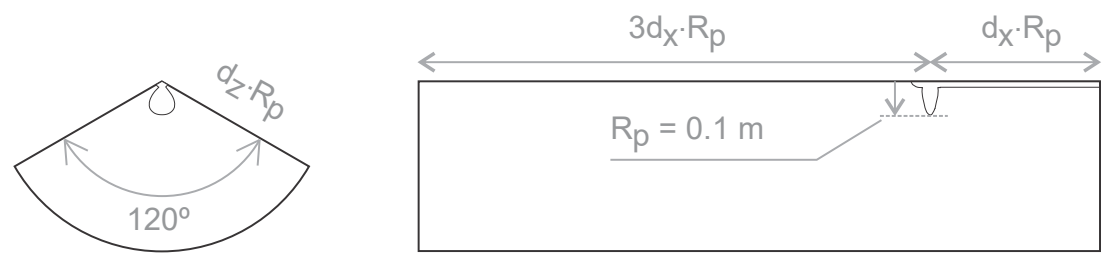

Source: Author

Figure 21 - Boundary conditions for propeller simulations

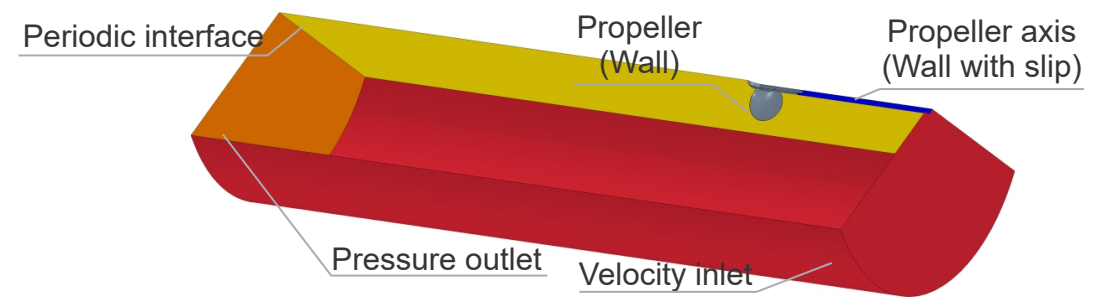

Source: Author

To standardize, all simulations are run in one node of a computational cluster, containing two processors of Intel Xeon X5560@2.80 GHz, totaling eight threads, with 24 GB DDR2 RAM. In this step, it is desired to isolate only the influence of domain size. For this, it is used the most refined mesh within estimated limits of the computational capacity of one node, so the level of discretization would not interfere in the results. This question is evaluated in detail in the next sections.

The simulations were done using Star-CCM+ software, steady-state, RANS with $k-\omega$ SST turbulence model and run until 5000 iterations were achieved. A high number of iterations is used as stopping criterion to guarantee its convergence by excess. An extreme case is chosen, with high rotation, $2400 \mathrm{RPM}$, and low advance velocity $J=0.3$, which leads to a high circulation zona and high thrust and required torque.

Figure 22 shows the results of 25 simulations as a function of domain size. The first plot shows the 1000-mean value of propeller efficiency; the second one, average time spent per iteration; and the third one, maximum physical memory (RAM) that was accumulated across all processes per iteration.

The values are divided by the results obtained for the largest domain $\left(d_{x}=d_{z}=8\right)$ and are shown in two two-dimensional graphs, Fig. 23 and Fig. 24, representing the projected view of the three-dimensional graph.

It can be noted from Fig. 23 that there are no significant improvements in propeller efficiency value with increasing $d_{x}$ from 5 to 8 , for all values of $d_{z}$. Also observing from Fig. 24, the propeller efficiency does not significantly improves by increasing $d_{z}$ from 5 to 8 , for all values of $d_{x}$. That is, increasing values of $d_{x}$ and $d_{z}$ from 5 to 8 have brought few 
Figure 22 - Propeller efficiency $\eta$ (left); time spent per iteration (center); and physical memory used (right) in function of domain dimensions
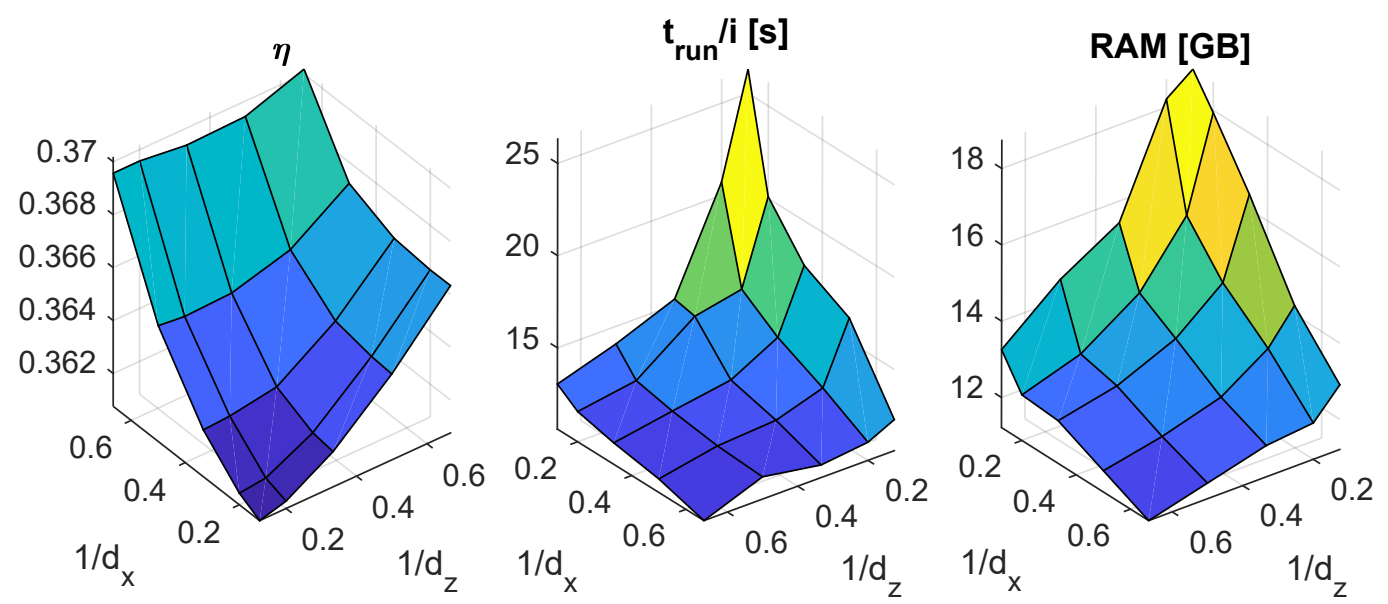

Source: Author

Figure 23 - Propeller efficiency $\eta$ (left); time spend per iteration (center); and physical memory used (right) in function of $d_{z}$ and normalized to the largest domain condition
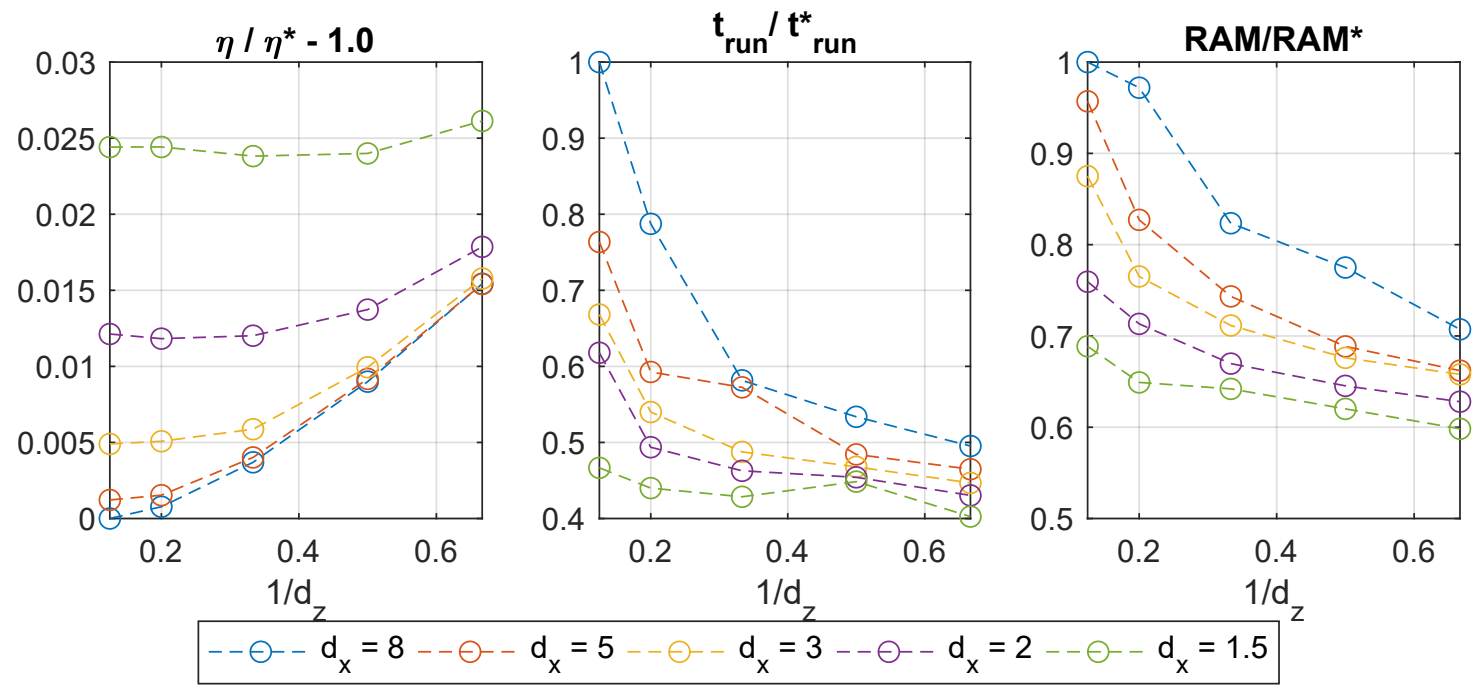

Source: Author

improvements in propeller efficiency value, with variations around $0.1 \%$.

The condition $d_{x}=d_{z}=5$ represents, in relation of condition of $d_{x}=d_{z}=8$, a decrease of $40 \%$ in time per iteration and a memory consumption reduction of $17 \%$, with a change in $\eta$ of $0.015 \%$ percent. Therefore, $d_{x}=d_{z}=5$ becomes an attractive candidate and is then adopted as the domain dimensions. 
Figure 24 - Propeller efficiency $\eta$ (left); time spend per iteration (center); and physical memory used (right) in function of $d_{x}$ and normalized to the largest domain condition

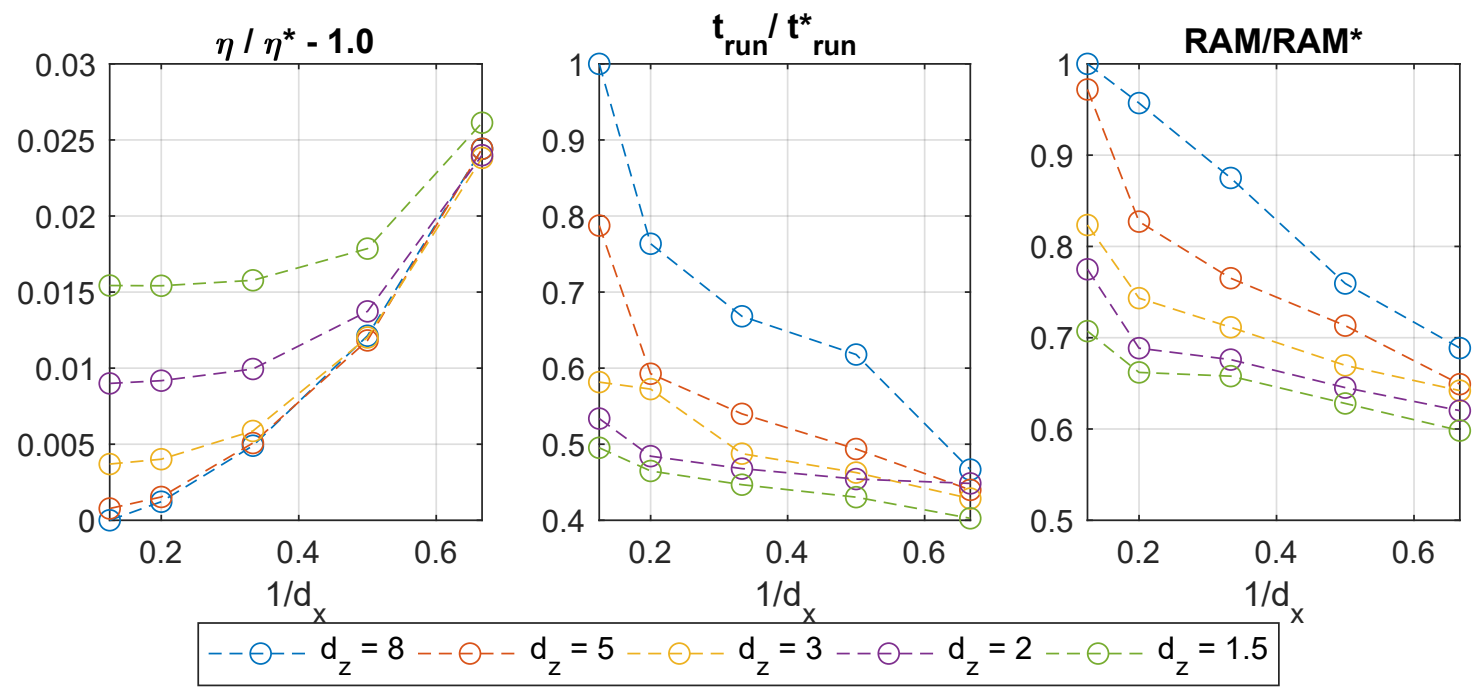

Source: Author

\subsection{Mesh Topology}

To determine which refinement level is appropriate, considering the accuracy and computational costs, two types of mesh, polyhedral and hexahedron, with three refinement levels each, are compared. All candidate meshes need to have the following characteristics: $y^{+}$close to 1.0; to represent the rounded geometry of the propeller tip (as presented in Sec. 6.1); and have an acceptable quality of the mesh, especially in terms of element aspect ratio and growing rate.

\subsubsection{Mesh refines}

Meshes have four volumes of refinement control, observable in Fig. 25.

Figure 25 - Geometry of refinement (shown the complete propeller. The model consist in $1 / 3$ of propeller) (left); section view (right)
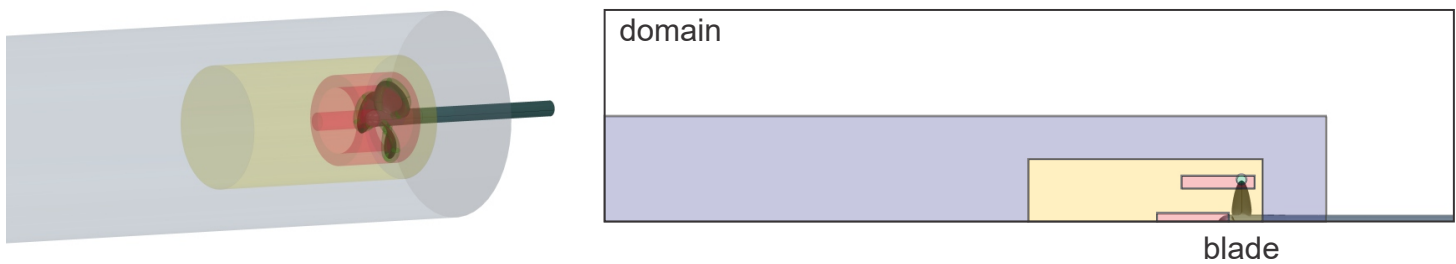

Source: Author

The first two volumes, in blue and yellow, are refinement due to propeller wake. The third one, in red, is composed of a hollow cylinder and a cylinder after the hub. These 
volumes refine the region of vortex detachment generated mainly in the propeller tip. The last one, in green, is a twisted cylinder that follows the propeller edge. This refinement changes smoothly the first element of the boundary layer, in order to maintain $y^{+}$near to 1.0 .

\subsubsection{Mesh candidates}

Two different mesh topologies and three refinement ratios are compared in one operational condition. These three mesh refinement ratios are based on their characteristic size, $h$, defined in Eq. (6.1), in which $N$ is the total number of elements and $V_{i}$, the element volume.

$$
h=\sqrt[3]{\frac{\sum V_{i}}{N}}
$$

As suggested by ASME (2009), it is desired that the ratio of characteristic sizes between meshes of different refines close to $\sqrt{2}$, as a good practice to estimate the discretization uncertainty of the solution. Table 1 shows the name of each mesh, number of elements, number of faces, characteristic size $h$, and ratio $r$ between its characteristic size and the characteristic size of the most refined mesh.

Table 1 - Candidate meshes

\begin{tabular}{cccccc}
\hline Name & Type & Number of elements & Number of faces & $h[\mathrm{~mm}]$ & $r$ \\
\hline hr1 & hexahedron & $10.8 \mathrm{M}$ & $32.1 \mathrm{M}$ & 3.64 & 1.00 \\
hr2 & hexahedron & $3.71 \mathrm{M}$ & $10.9 \mathrm{M}$ & 5.20 & 1.43 \\
hr3 & hexahedron & $1.38 \mathrm{M}$ & $4.02 \mathrm{M}$ & 7.24 & 1.98 \\
pr1 & polyhedral & $10.7 \mathrm{M}$ & $64.8 \mathrm{M}$ & 3.65 & 1.00 \\
pr2 & polyhedral & $3.85 \mathrm{M}$ & $21.2 \mathrm{M}$ & 5.14 & 1.41 \\
pr3 & polyhedral & $1.39 \mathrm{M}$ & $6.83 \mathrm{M}$ & 7.21 & 1.98 \\
\hline
\end{tabular}

Figure 26 shows a comparative of volumes between the six candidates.

There is attention that the last element of prism layer dimensions was similar to its adjacent elements, ensuring good continuity of mesh. For this, the software-defined scalar element volume change, which describes the ratio between the element volume and its largest adjacent one, was observed. The value of 1.0 indicates an element that has the same volume as its adjacent one, and it is the ideal value. According to the software developer, values below 0.01 indicate bad elements.

Figure 27 shows that the rounded tip feature is represented in all six meshes. The volume change can also be observed in Fig. 27, noting that element quality presents satisfactory values in the last elements of the prism layer, indicating a smooth transition. 
Figure 26 - Comparative of the element volumes for propeller simulations

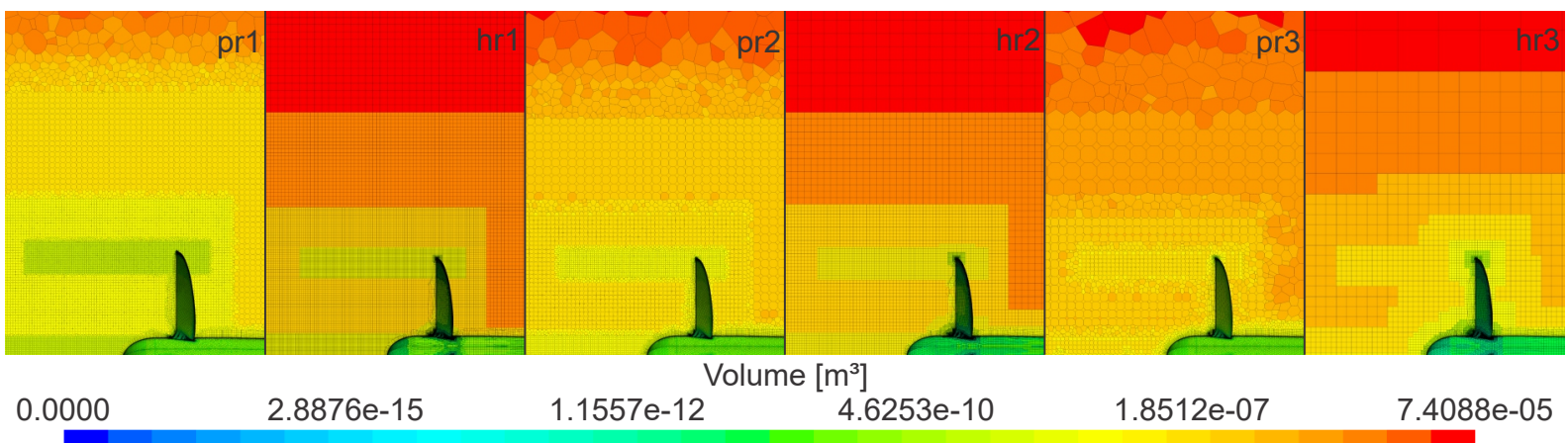

Source: Author

Figure 27 - Detail of mesh at propeller tip

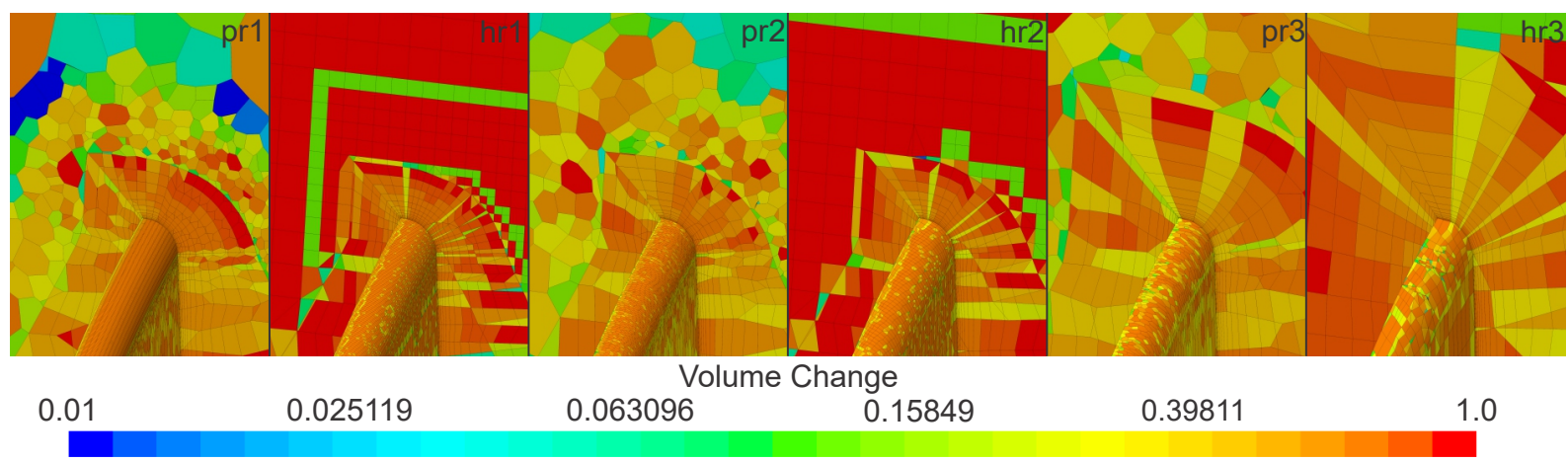

Source: Author

It is also used the pre-defined scalar element quality, that defines how deformed the element is compared to one of the perfect dimensions. Table 2 shows the results for candidate meshes. All 6 meshes have $100 \%$ of their elements with good quality. In the case of quality related to volume change, all six meshes have values very close to $100 \%$. Besides, it is noted that the percentage of good elements grows with the number of elements.

Table 2 - Candidate meshes

\begin{tabular}{ccc}
\hline Name & Acceptable volume change & Acceptable element quality \\
\hline hr1 & $99.999 \%$ & $100 \%$ \\
hr2 & $99.997 \%$ & $100 \%$ \\
hr3 & $99.970 \%$ & $100 \%$ \\
pr1 & $99.999 \%$ & $100 \%$ \\
pr2 & $99.979 \%$ & $100 \%$ \\
pr3 & $99.969 \%$ & $100 \%$ \\
\hline
\end{tabular}

Results show that both mesh topologies are acceptable in terms of mesh quality. 


\subsubsection{Results and Verification}

In order to evaluate the performance of the candidate meshes, one operation condition is simulated with advance velocity of $3.029 \mathrm{~m} / \mathrm{s}$ and rotation velocity of $1608 \mathrm{RPM}$, using steady-state condition and without cavitation model. The stopping criteria are, in the last 1000 iterations, the difference of oscillation of $K T$ values must be below $1 \times 10^{-4}$, while for $K Q$, below $1 \times 10^{-5}$. These values are present an error of the order of magnitude lower than that obtained by the mesh refining difference, as recommended by ASME (2009).

To standardize, almost all simulations are run in one node of a computational cluster (the same of Sec. 6.2). The only exception is the mesh pr1, the most refined polyhedral mesh. This mesh cannot be done within 24 GB RAM, so eight threads of Intel Xeon E5-2630v3 @ 2.40 GHz with 128 GB DDR3 RAM were used. It should be noted that this processor is newer and has a better performance per thread.

The mesh comparison is made through the analysis of discretization uncertainty, computational cost evaluated by time, the curve of convergence, and a qualitative criterion through the visual comparison of the vortex formation.

\subsubsection{Discretization Uncertainty}

Based on three refinements of each type of mesh, the discretization uncertainty is estimated through the Verification and Validation $(\mathrm{V} \& \mathrm{~V})$ procedure proposed by ASME (2009). Figure 28 shows the average of the last 1000 iterations of $K T$ and $K Q$ as a function of $r$. Richardson extrapolation is also shown in solid line as a function of the refining ratio and, in dashed line, the estimated uncertainty with $95 \%$ confidence interval.

Figure 28 - Comparison of thrust and torque coefficients in function of the refining ratio

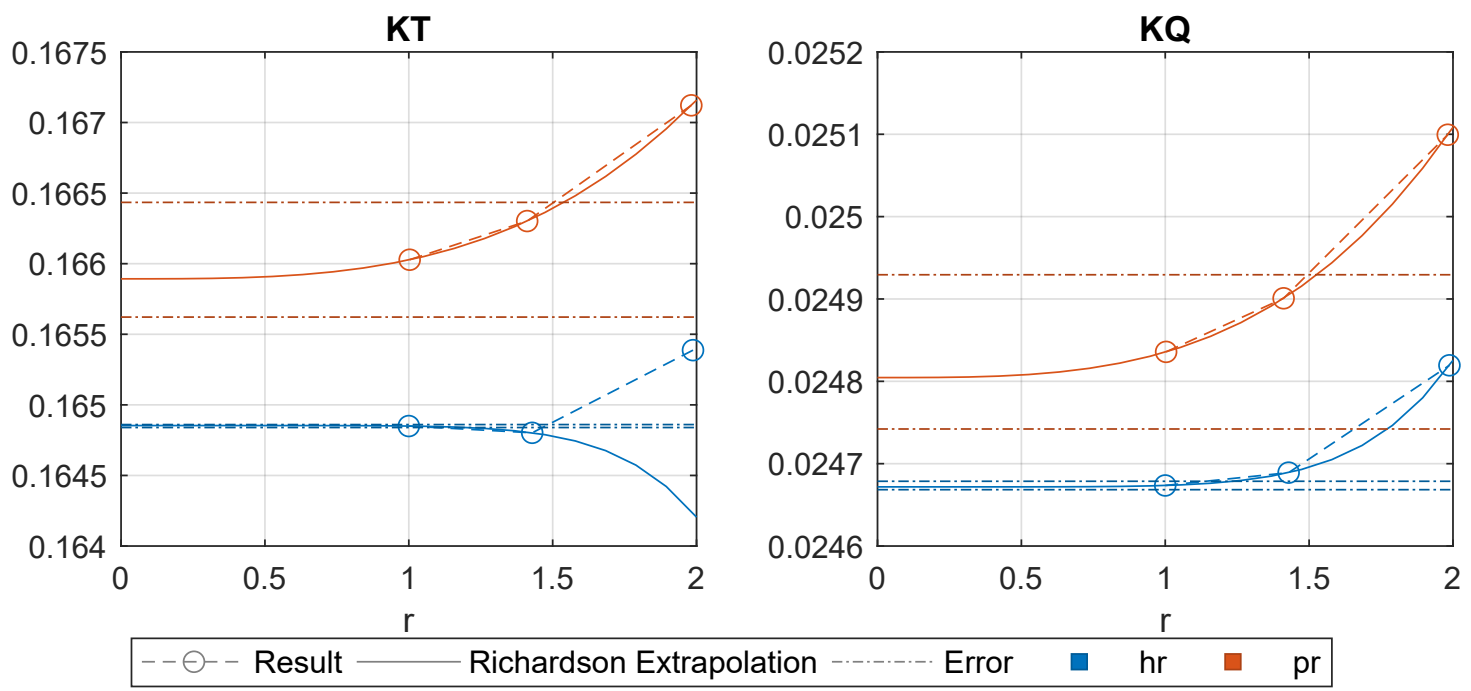


There was a great variation of results for mesh r3 to $r 2$. However, it is notably smaller between mesh $r 2$ and $r 1$. For hexahedron meshes, this variation was smaller compared to polyhedral meshes, which results in a smaller error estimation, as noted by Fig 28.

\subsubsection{Computational costs}

Figure 29 shows the time spent in each candidate meshes. It is possible to conclude that polyhedral meshes spend more time for all activities, even the pr1, compared to $\mathrm{hr} 1$, in which is used a more powerful processor.

Figure 29 - Time to generate the mesh (left); to satisfy the stopping criteria (center); and time per iteration (right)
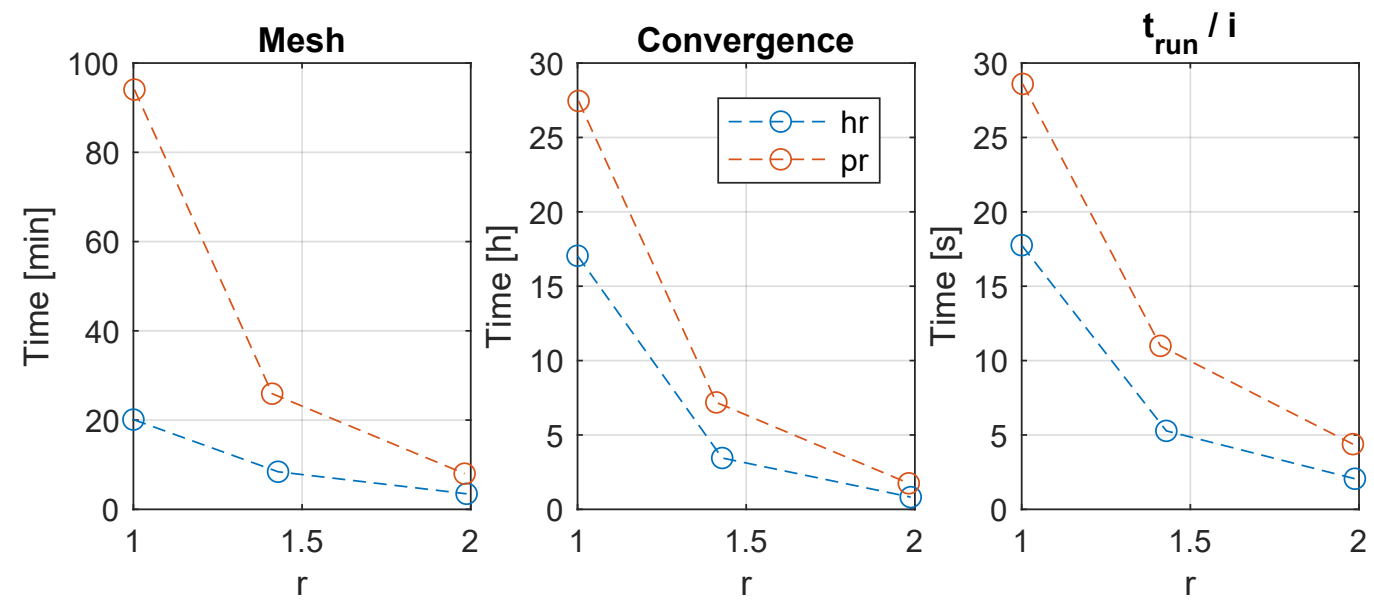

Source: Author

\subsubsection{Curve of convergence}

Figure 30 and Fig. 31 show the evolution of the coefficients, in which it is possible to observe the required iterations to achieve the stopping criteria. The more refined the mesh, the more iterations take to reach the stopping criteria. In addition, a slower behavior is observed in the change of the coefficients along with the iterations.

\subsubsection{Q-criterion}

Another influence of mesh refinement is observed visually in Fig. 32 by the Qcriterion, defined by:

$$
Q_{\text {crit }}=\frac{1}{2}\left(\|\boldsymbol{\Omega}\|^{2}-\|\mathbf{D}\|^{2}\right)
$$

in which $\Omega$ is the vorticity tensor. In other words, Q-criterion highlights vorticities that have small deformation (JEONG; HUSSAIN, 1995). In this sense, boundary layer regions are penalized by the Q-criterion. 
Figure 30 - Evolution of $K T$ (left); and absolute difference of the coefficient with its previous iteration (right)

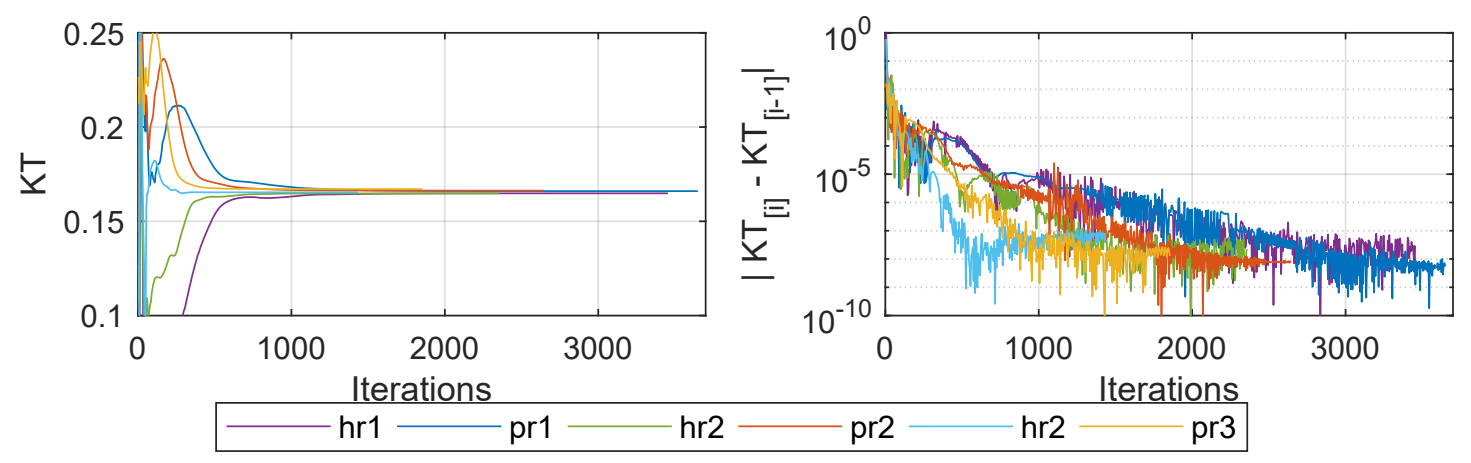

Source: Author

Figure 31 - Evolution of $K Q$ (left); and absolute difference of the coefficient with its previous iteration (right)

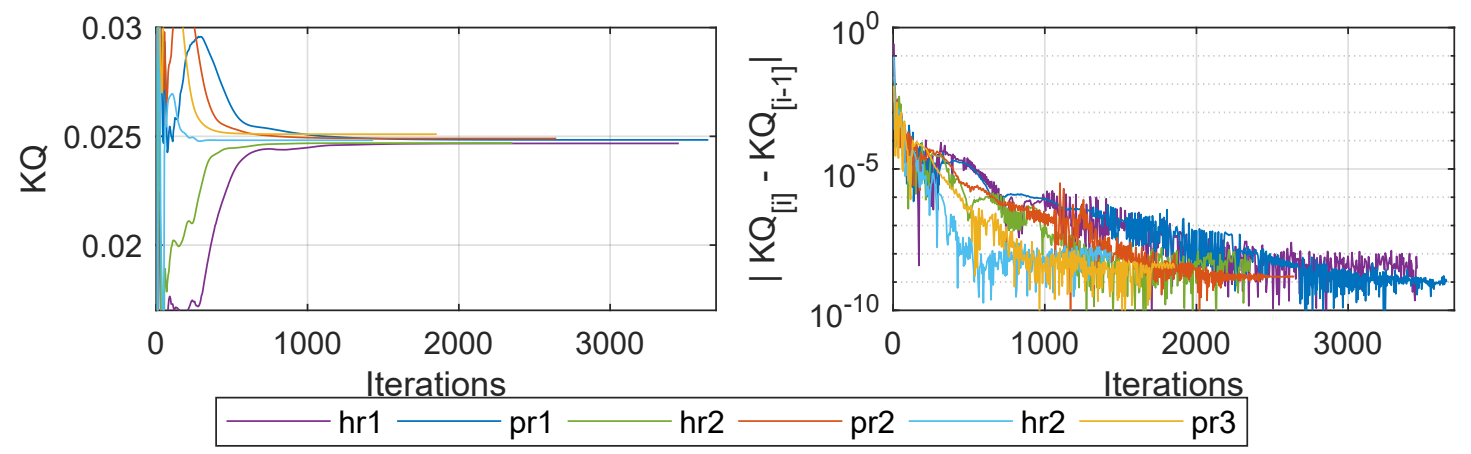

Source: Author

Figure 32 shows the Q-criterion iso-surface in the hexahedron and polyhedral meshes. For the same refinement index, hexahedron meshes model a longer Q-criterion iso-surface length compared to polyhedral ones.

\subsubsection{Discussion}

As the hexahedron mesh had a better prediction of the Q-criterion, lower uncertainty and cost than the polyhedral one, it is used for this work. Considering the computational cost, the hr2 produced results close to the hr1 one and with acceptable computational costs. Therefore, the $\mathrm{hr} 2$ mesh is used to investigate the turbulence model and to simulate several conditions of open-water simulations.

\subsubsection{Mesh for Optimization Framework}

This section presents the mesh used in the optimization framework. It should present all requirements with the lowest number of elements as possible, to ensure a fast evaluation of cost function. 
Figure 32 - Q-criterion of $200 \mathrm{~s}^{-2}$

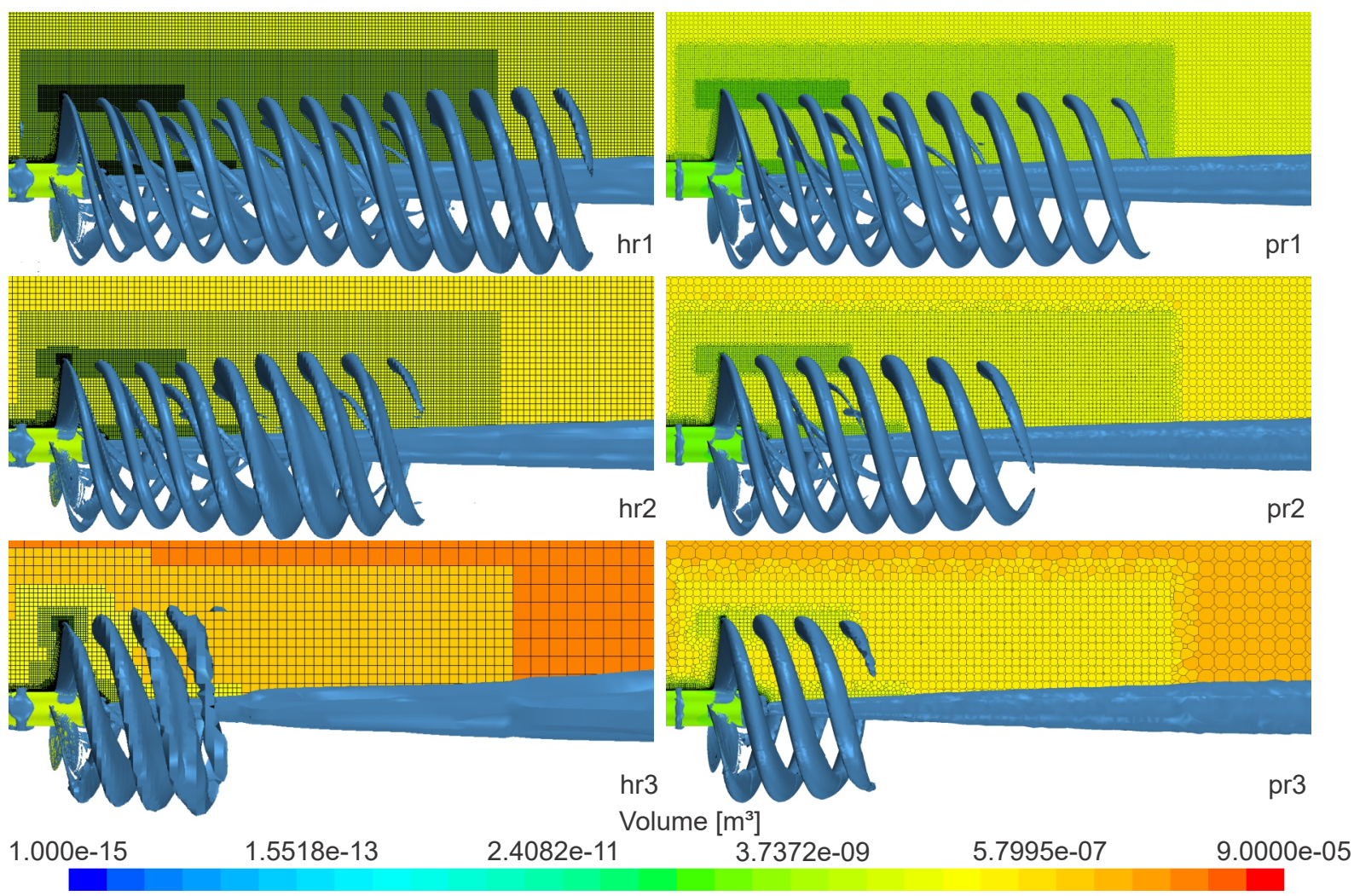

Source: Author

Adjoint methods require a small residual. To ensure this by using coupled solver, it is important to ensure a good quality of the mesh. Otherwise, it would lead to convergence issues.

Despite the choice for the hexahedron topology in the last sub-section, a polyhedral mesh is used, following recommendations of Star-CCM+ for adjoint solver (Siemens, 2017). Moreover, for coarse mesh, it is easier to ensure good quality with few elements when a polyhedral topology is adopted.

Figure 33 shows the mesh used in the optimization framework. All the previous presented requirements are also imposed here, such as representing propeller tip and wall $y^{+}$close to 1.0, and the volumes for refinement control.

Comparing the chosen hexahedron and the optimization meshes, Fig. 34 shows the difference regarding mesh topology, while Tab. 3 indicates some particulars comparatives. 
Figure 33 - Optimization mesh topology, detail on propeller tip and Q-criterion of $200 \mathrm{~s}^{-2}$
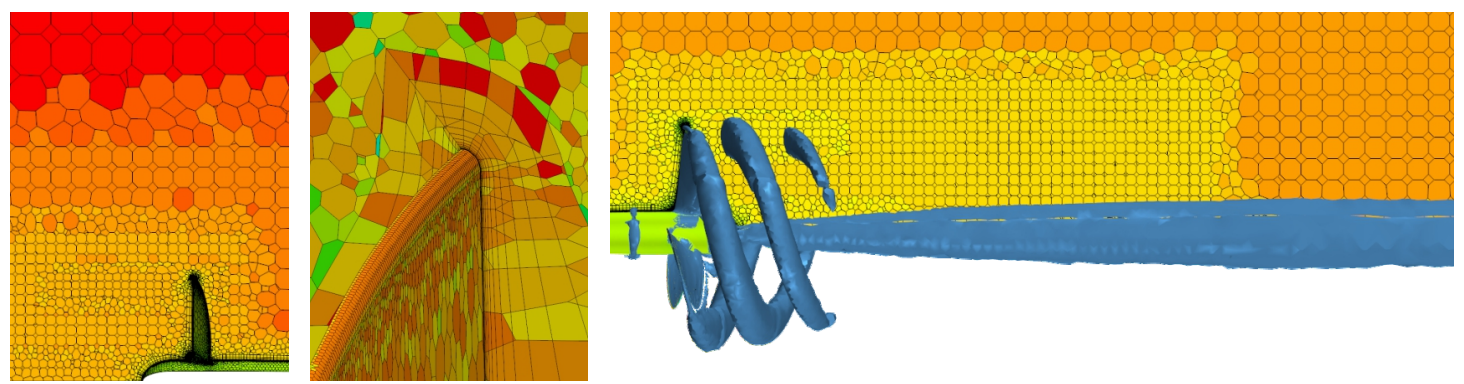

Source: Author

Figure 34 - Comparative between coarse mesh (used in optimization framework) (left) and $\mathrm{hr} 2$, hexahedron mesh
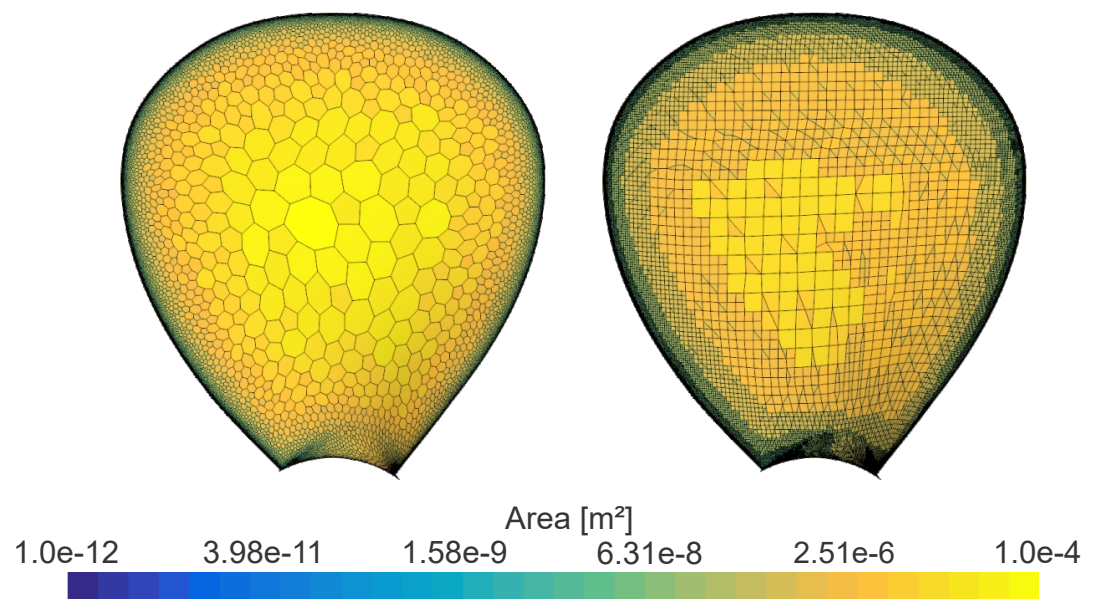

Source: Author

Table 3 - Dimensions of numerical model of cavitation tunnel.

\begin{tabular}{lcc}
\hline Variable & Polyhedral/Coarse & Hexahedron/Fine \\
\hline Volume elements & $8.39 \times 10^{5}$ & $3.71 \times 10^{5}$ \\
Elements on blade & $3.69 \times 10^{4}$ & $9.61 \times 10^{4}$ \\
Acceptable Volume change & $100 \%$ & $99.997 \%$ \\
Acceptable element quality & $100 \%$ & $100 \%$ \\
\hline
\end{tabular}

\subsection{Turbulence Model}

This section analyzes the influence of the turbulence model in order to predict the hydrodynamic characteristics best.

Table 4 shows cases $\mathrm{C} 1$ and $\mathrm{C} 2$, which are low Reynolds cases. On the other hand, Tab. 5 shows cases with high Reynolds, C3 and C4. Cases C2 and C3 represent those with a close values of the advance ratio.

Four turbulence models are compared: Spalart-Allmaras (SA); $k-\omega \mathrm{SST}(\mathrm{SST})$; 
$k-\omega$ SST with low Reynolds number correction (SST LowRe); and Realizable $k-\epsilon$ $($ R. $k-\epsilon)$. Model details are discussed in Sec. 2.4.

Table 4 - Turbulence model comparative - low Reynolds numbers

\begin{tabular}{ccccccc}
\hline Condition & & $\mathrm{C} 1$ & & & $\mathrm{C} 2$ & \\
$v_{a}[\mathrm{~m} / \mathrm{s}]$ & & 0.571 & & & 1.116 & \\
$n[\mathrm{RPM}]$ & & 805 & & & 804 & \\
\hline coeff. & $K T$ & $K Q$ & $\eta$ & $K T$ & $K Q$ & $\eta$ \\
\hline Exp. & 0.330 & 0.0433 & 0.231 & 0.241 & 0.0341 & 0.421 \\
SA & 0.317 & 0.0419 & 0.256 & 0.229 & 0.0323 & 0.471 \\
SST & 0.316 & 0.0417 & 0.256 & 0.229 & 0.0321 & 0.472 \\
SST LowRe & 0.316 & 0.0418 & 0.256 & 0.229 & 0.0322 & 0.471 \\
R. $k-\epsilon$ & 0.317 & 0.0419 & 0.256 & 0.229 & 0.0322 & 0.471 \\
\hline
\end{tabular}

Table 5 - Turbulence model comparative - high Reynolds numbers

\begin{tabular}{ccccccc}
\hline Condition & & $\mathrm{C} 3$ & & & $\mathrm{C} 4$ & \\
$v_{a}[\mathrm{~m} / \mathrm{s}]$ & & 3.367 & & & 5.500 & \\
$n[\mathrm{RPM}]$ & & 2407 & & & 2426 & \\
\hline coeff. & $K T$ & $K Q$ & $\eta$ & $K T$ & $K Q$ & $\eta$ \\
\hline Exp. & 0.244 & 0.0341 & 0.429 & 0.112 & 0.0203 & 0.582 \\
SA & 0.231 & 0.0319 & 0.484 & 0.115 & 0.0191 & 0.652 \\
SST & 0.231 & 0.0317 & 0.487 & 0.115 & 0.0187 & 0.664 \\
SST LowRe & 0.231 & 0.0317 & 0.486 & 0.115 & 0.0188 & 0.658 \\
R. $k-\epsilon$ & 0.231 & 0.0316 & 0.488 & 0.115 & 0.0190 & 0.655 \\
\hline
\end{tabular}

It is observed that the difference between models is, in most cases, null. Even varying the Reynolds number, there is no significant difference in coefficients. Thus, the SST model is chosen based on its extense application in the literature, as discussed in Sec. 2.4 .

\subsection{Cavitation}

Although it is not intended to perform the topology optimization with cavitation modeling, it is intended to develop a CFD method that can accurately predict the propeller performance, even with the cavitation model. A well-defined method reduces the use of a cavitation tunnel and is used to verify the Topology Optimization results.

The propeller performance curves are analyzed for cavitation numbers o 1 and 3, respectively presented as $\sigma=1$ and $\sigma=3$. Simulations without cavitation are presented by $\sigma=\infty$. 
For simulations without cavitation effects, the same methodology developed in previous steps is performed, such as steady-state formulation and stopping criteria.

Because cavitation simulations are not a stationary phenomenon, the implicit unsteady formulation is adopted. A constant time step of $1 \times 10^{-4} \mathrm{~s}$ is used in all operating conditions with $1 \mathrm{~s}$ physical time of stopping criterion. At each time step, five inner iterations were done, resulting in a total of 5000 iterations per simulation. The time step of $1 \times 10^{-4}$ s ensures a volumetric mean of the Courant number less than 1.0 for all simulated conditions. For example, simulation with the advance velocity of $4.851 \mathrm{~m} / \mathrm{s}$ and rotation of 2103 RPM, the highest combination of advance velocity and rotation, has a volumetric mean of Courant number of 0.8649 . It is noted for all simulations that $1 \mathrm{~s}$ is sufficient physical time to achieve a constant harmonic condition.

\subsection{Experimental Values Comparative and Discussions}

The obtained results with the developed numerical methodology are compared in Fig. 35 with the experimental results obtained in the Cavitation Tunnel at the Institute for Technological Research (IPT). The experimental procedures are explained in detail in Sec. B.

The results show good consistency of the values obtained numerically with those obtained experimentally at the IPT's Cavitation Tunnel, including with cavitation effects. It is shown that, even in conditions in which cavitation is predominant the results have a good correlation with those obtained experimentally.

Defined the methods that showed good accuracy with experimental results, it can apply for simulations of propellers with hydrophobic effects, using the same domain dimensions, mesh and turbulence model, expecting similar accuracy as obtained in this chapter. 
Figure 35 - Thrust coefficient $K T$ versus advance ratio $J$ and Cavitation pattern of numerical results (Iso-surface of 0.5 volume fraction of water) (top); Torque coefficient (bottom left); and Propeller efficiency (bottom right)
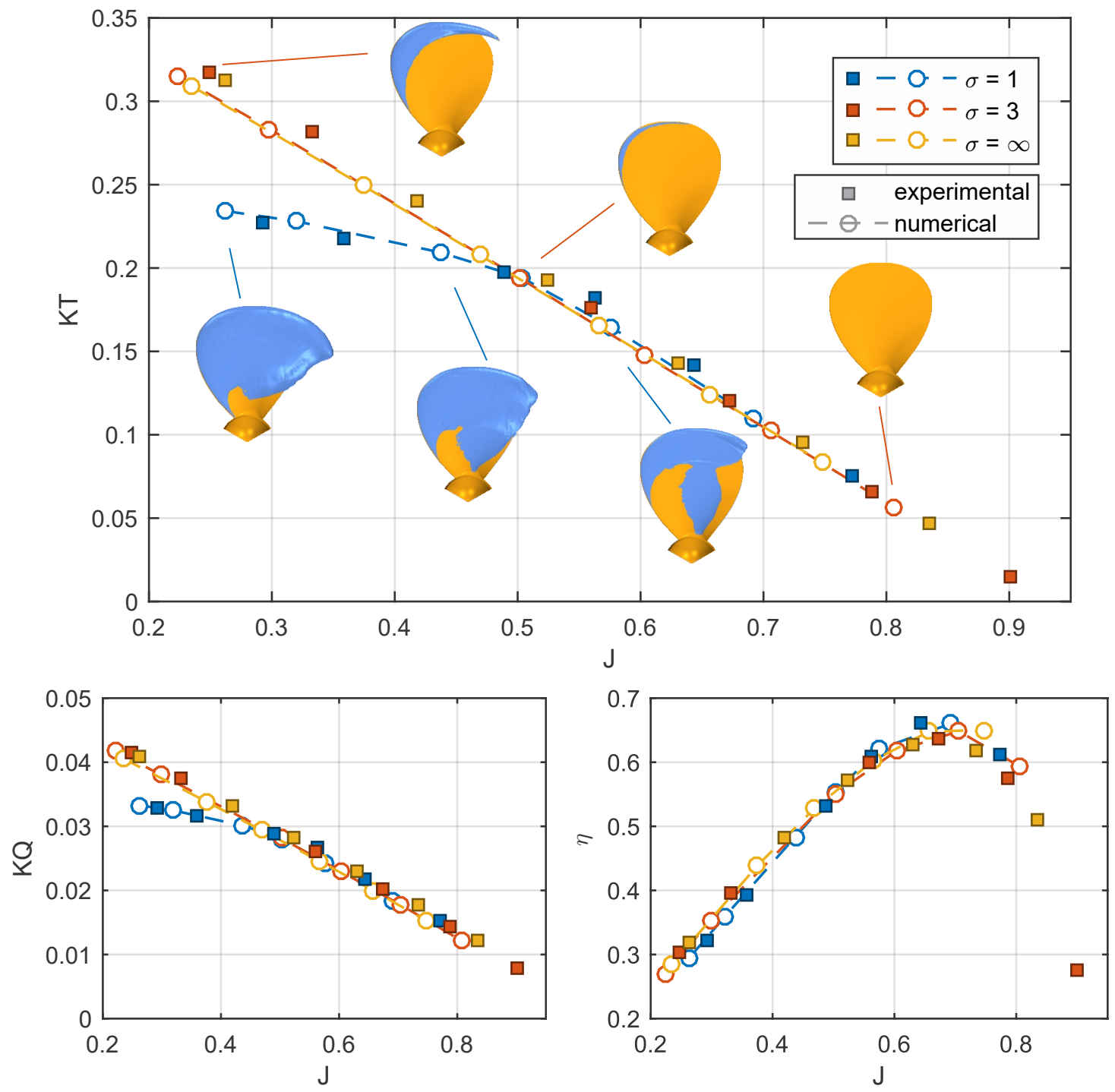

Source: Author 



\section{Numerical Analysis and Results of Hy- drophobic Simulations}

With a defined method for propeller simulation, a numerical analysis based on RANS CFD software is done to model the behavior of the boundary layer flow with the boundary condition that imposes the low friction / hydrophobic effect. The objective is to analyze the trade-off between the skin-friction drag reduction due to SHS presence, an expected result, and the increase of suction pressure, i.e., reduction of absolute pressure, which may increase the cavitation phenomena. This chapter, published in Katsuno, Dantas and Silva (2018), aims to estimate the gain of performance in propellers, guiding the definitions and restrictions that should be looked for topology optimization, as well as being necessary for a correct definition for the cost function.

\subsection{D Hydrofoil Comparatives}

\subsubsection{Hydrofoil geometry}

The 2D study is carried out with two different styles of hydrofoil: NACA0015 and Ogival hydrofoils, shown in Fig. 36. NACA0015 is chosen because it is commonly used in the naval field, such as in rudders and keels. The ogival foil is contained in a Gawn-Burril (KCA) propeller, described in Sec. 6.1. This foil is present in the $70 \%$ of propeller H1 radius, which is used in the 3D study. Ogival foils have lower hydrodynamic performance, but have more uniform pressure distribution, reducing the cavitation phenomena.

Figure 36 - Foil NACA0015 (left); and ogival foil (right)
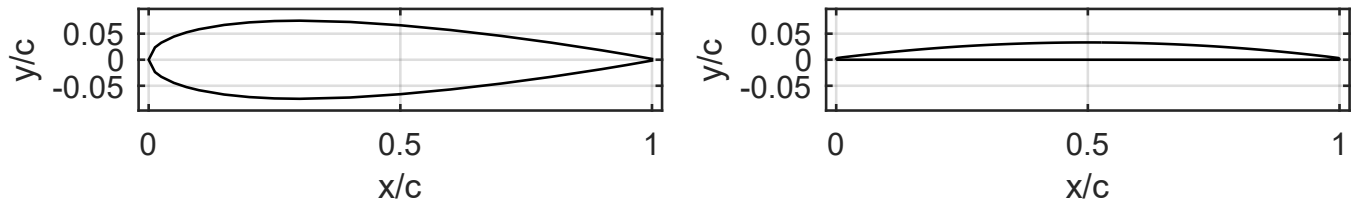

Source: Author

\subsubsection{Domain Geometry and Boundary Conditions}

For the two-dimensional hydrofoil simulations, the domain geometry is rounded at the input zone, as shown in Fig. 37, which also shows the dimensions and boundary conditions. The domain dimensions are large enough so that their edges do not significantly interfere in hydrofoil flow. There is a concern that the flow wake ends in a proper boundary 
condition (pressure outlet). As it is shown in Fig. 37, the mesh refinement had a spread angle higher than the highest angle of attack that is simulated. This mesh refinement ends inside of pressure outlet boundary conditions, not crossing with the upper and lower part of the domain.

Figure 37 - Domain geometry and boundary conditions for foil simulations

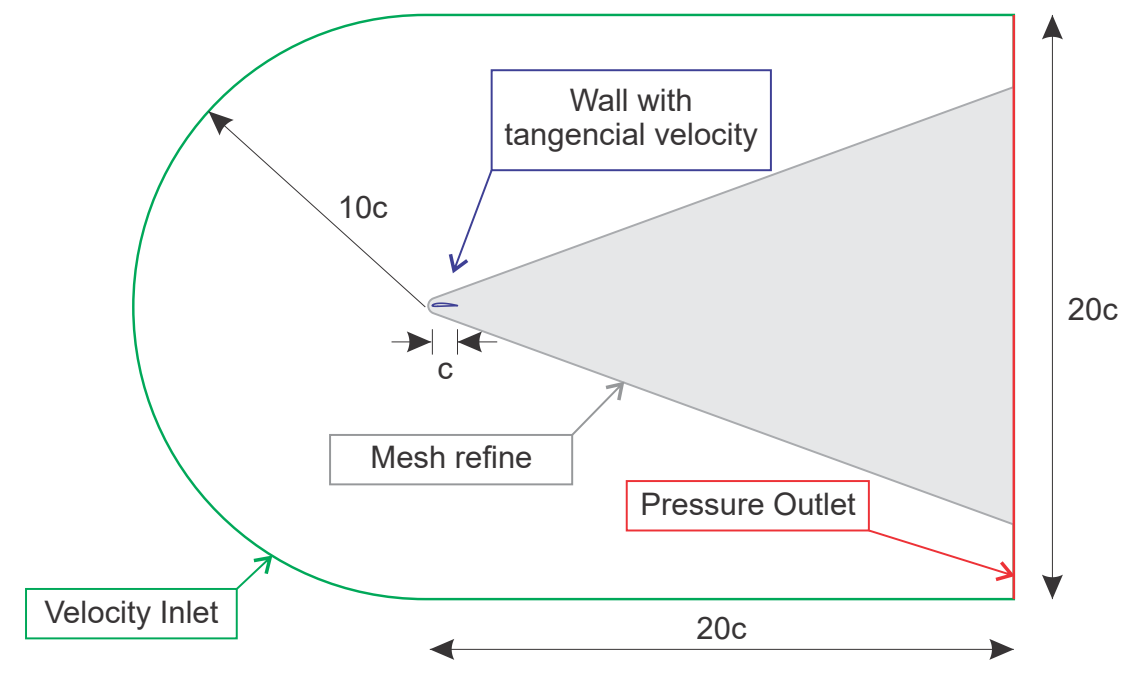

Source: Author

\subsubsection{Mesh Topology}

It is intended to create a polyhedral mesh with enough elements to correctly represent the hydrodynamic behavior with an accessible computational cost. The first element of the prism layer was such that $y^{+}$must be close to 1.0. The prism layer stretching is 1.3 , and the last element of the prism layer has dimensions close to its non-prism layer neighbor.

The mesh is refined close to wall and behind the foil, as shown in Fig. 37, in order to correctly model the wake. Figure 38 shows the mesh used for the ogival simulations only, once the NACA0015 has a similar mesh topology. 
Figure 38 - Mesh used for ogival foil simulations

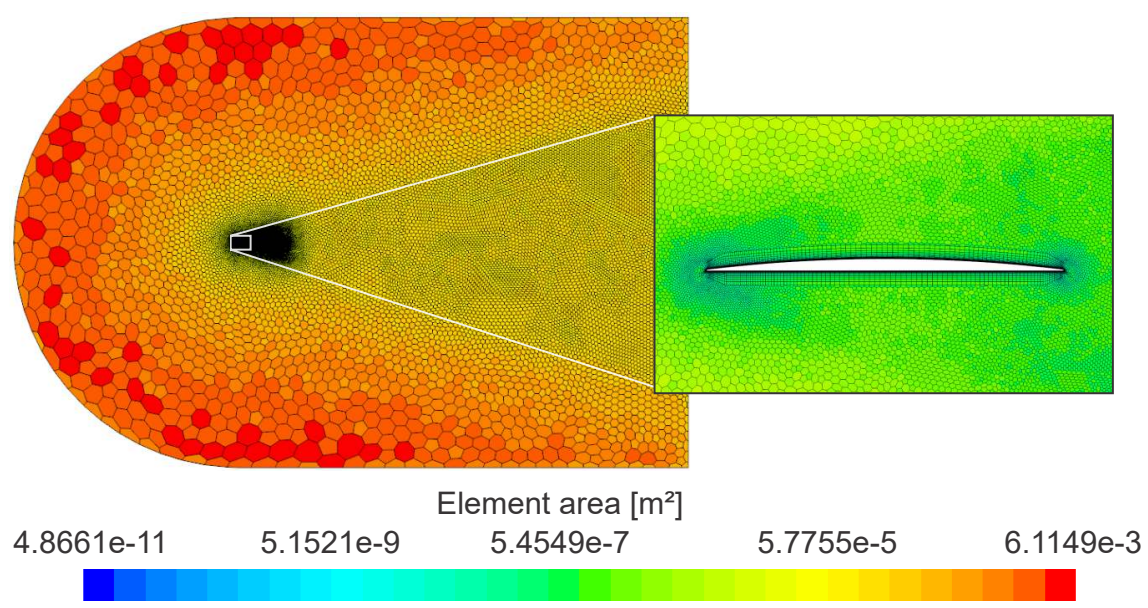

Source: Author

\subsection{Simulation conditions}

Several simulations are performed, varying the angle of attack, but below the stall condition. Four different levels of hydrophobicity are compared, varying the slip length $b$, with a no-slip condition. These tested slip lengths were based on values found in the referenced papers, such as in Min and Kim (2004).

The chord $c$ in both foils is $0.07615 \mathrm{~m}$, which represents the chord of the propeller at the position of $70 \%$ of its radius. The far-field velocity is $12.3 \mathrm{~m} / \mathrm{s}$. The value is an estimate of resulting velocity at $70 \%$ of the radius with a rotational speed of $1600 \mathrm{RPM}$ and velocity such that result in high propeller efficiency, close to $J=0.7$. This estimate of velocity essentially consists of the sum of the tangential and advance velocity, without considering the induced velocity.

\subsection{Hydrofoil Results}

\subsubsection{Lift and Drag Coefficients}

Figure 39 shows the lift and drag coefficients in function of angle of attack and slip length for NACA0015 foil, and Fig. 40, for ogival foil. The effect of hydrophobia for NACA0015 has a small influence on the lift but a remarkable influence on drag. This result is expected, since hydrophobicity is related to shear stress, directly affecting the viscous drag. Also, the lift to drag ratio increases considerably as the slip length increases. 
Figure 39 - Lift (left); drag coefficients (center); and drag polar of NACA0015 foil (right)
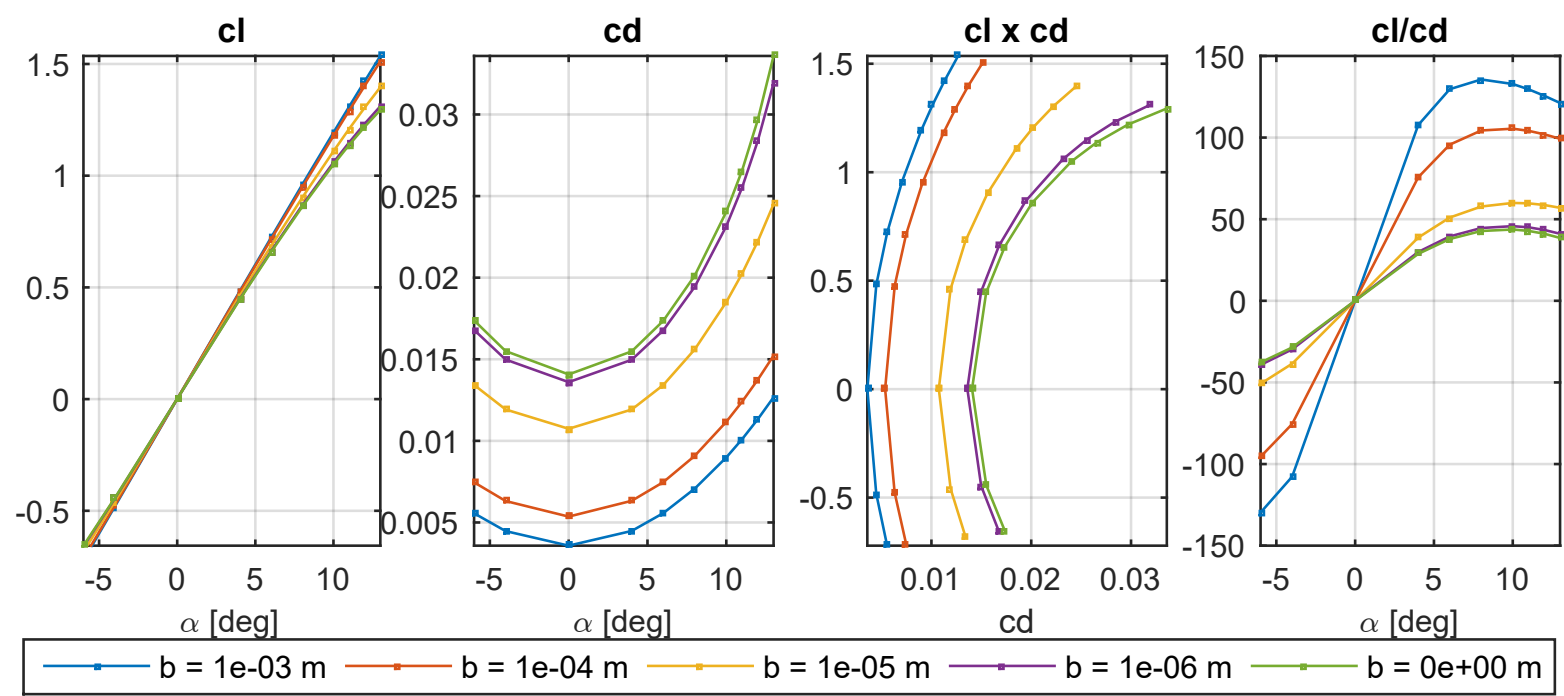

Source: Author

Figure 40 - Lift (left); drag coefficients (center); and drag polar of Ogival foil (right)
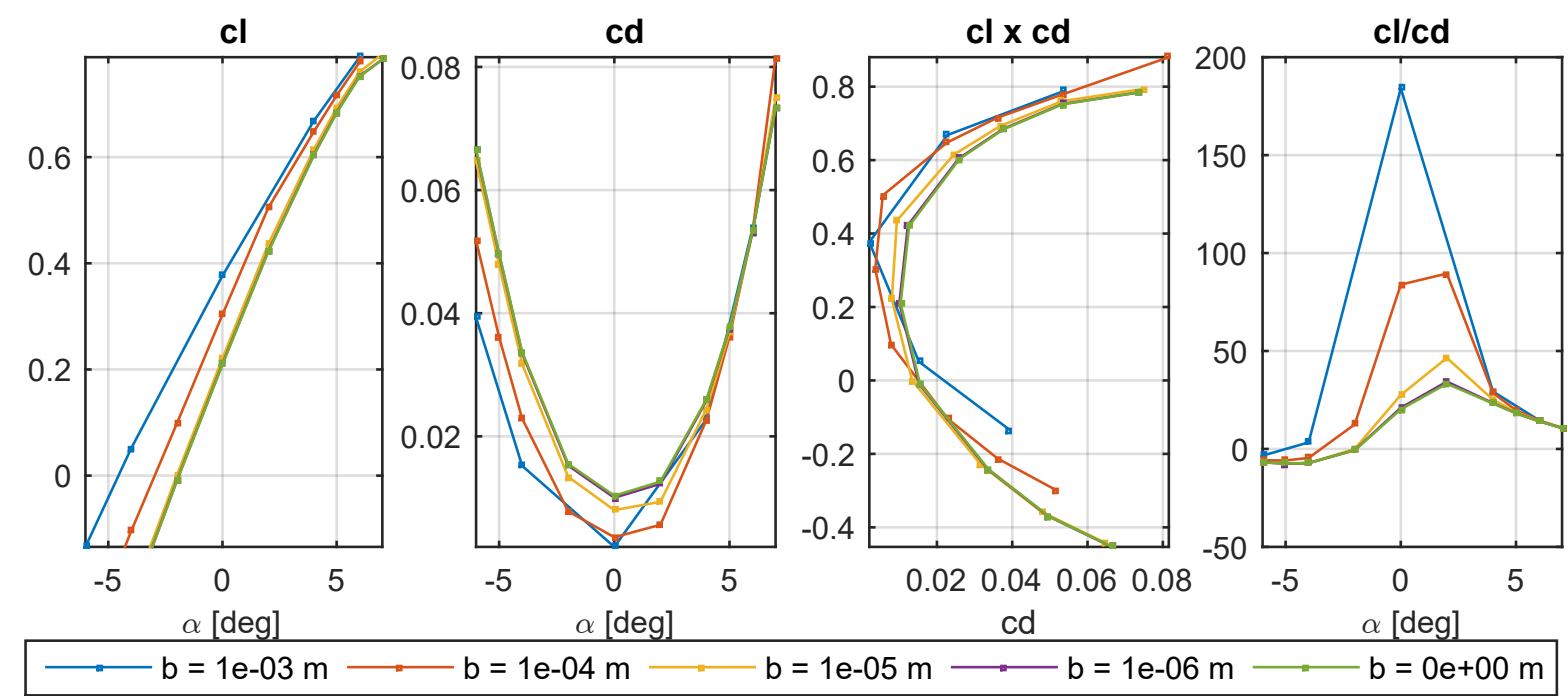

Source: Author

\subsubsection{Skin Friction Coefficient}

The skin friction coefficient distribution along the chord for NACA0015 foil is shown in Fig. 41, and for ogival foil, in Fig. 42. The effect of hydrophobicity stronger influences the $C f$ distribution, and it is noted that the longer the slip length, the lower the skin friction, for both low and high angle of attack. 
Figure 41 - Skin Friction coefficient distribution for NACA0015 foil

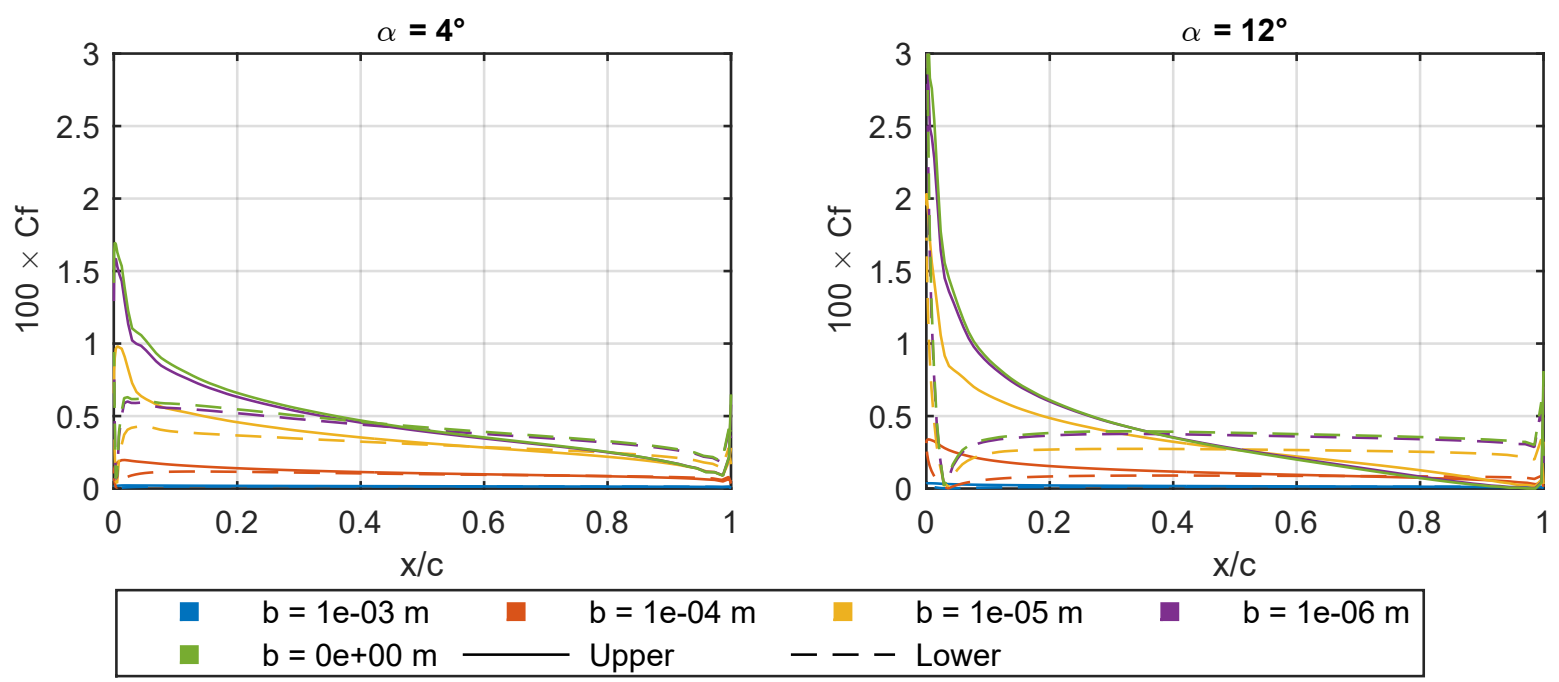

Source: Author

Figure 42 - Skin Friction coefficient distribution for Ogival foil

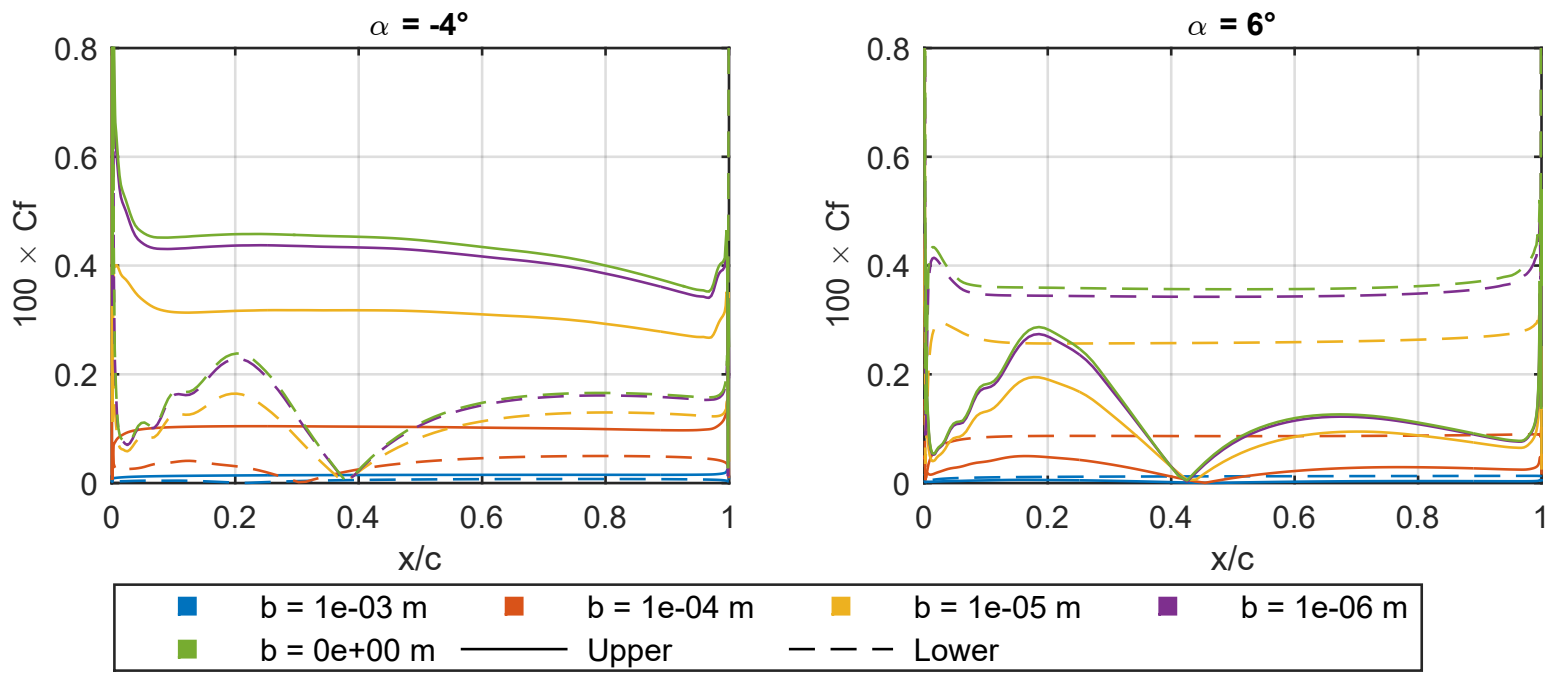

Source: Author

\subsubsection{Pressure Coefficient}

Figure 43 shows the pressure coefficient distribution along the chord for NACA0015 foil. For $\alpha=4^{\circ}$, as observed previously, the lift is almost the same for several slip lengths. As the contribution of the lift is mostly due to pressure, it is expected that the pressure distribution for several slip lengths is close. For $\alpha=12^{\circ}$, it can be noted that as the slip length increases, the peak of suction pressure increases too, which could lead to higher 
cavitation.

Figure 43 - Pressure coefficient distribution of NACA0015 foil

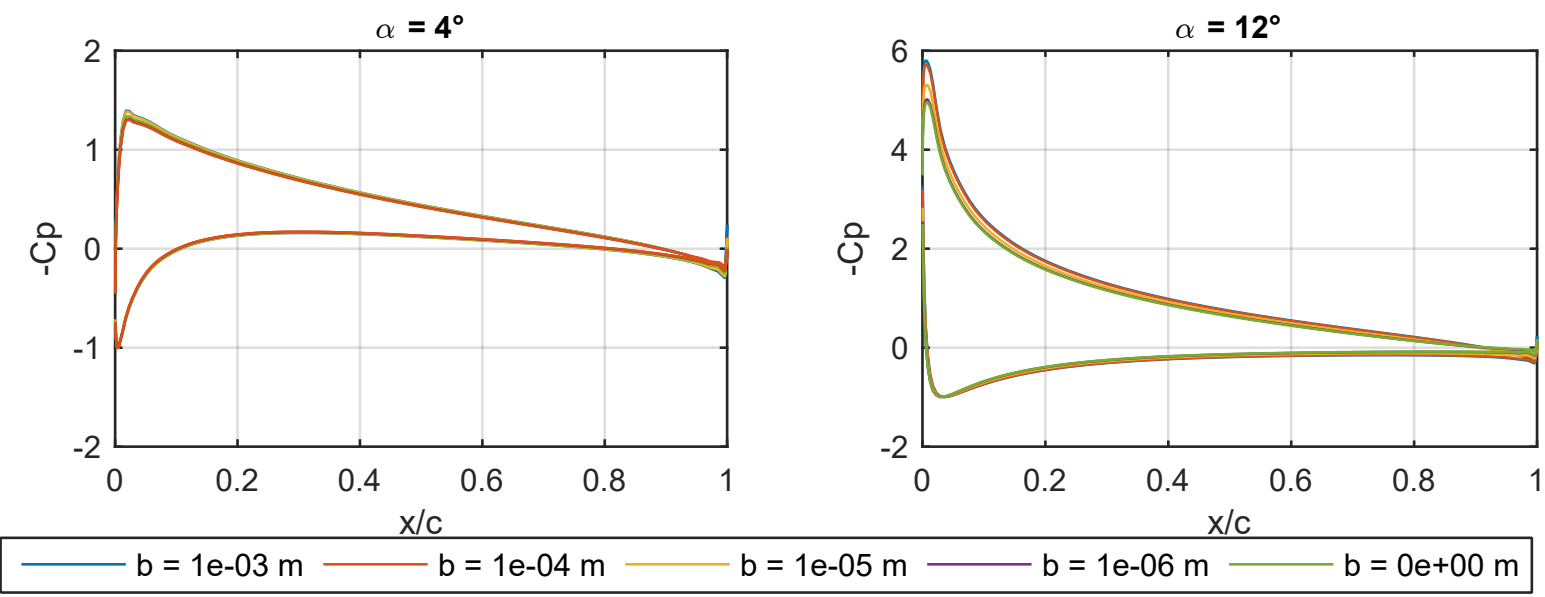

Source: Author

For the ogival foil, the pressure coefficient along the chord is observed in two conditions: $\alpha=-4^{\circ}$ and $\alpha=6^{\circ}$, shown in Fig. 44. It is noted that the curves are close to each other, with a certain difference for the most extreme case of simulated hydrophobicity, for $\alpha=-4^{\circ}$. For $\alpha=6^{\circ}$, the curves of $C p$ are practically the same. It is also noted that there is no suction pressure peak, such as the NACA0015.

Figure 44 - Pressure coefficient distribution of ogival foil

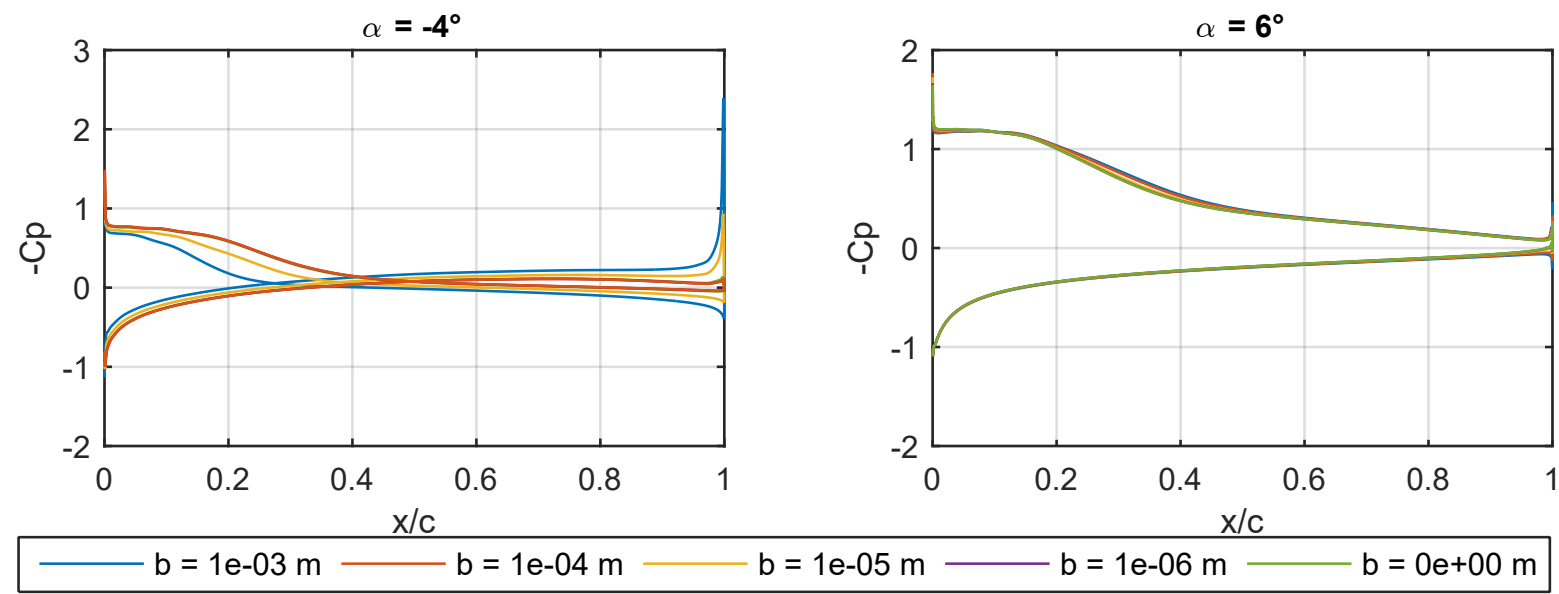

Source: Author

No significant differences are observed but, as the slip length increases, the suction pressure peak increases too, which could lead to higher cavitation. Figure 45 shows the fraction of chord that has $C p$ less than a given value, in order to investigate the effect of these peaks. Each point in the figure represents a different slip length. 
Figure 45 - Fraction of chord with $C p<C p_{\min }$ in function of lift-to-drag ratio

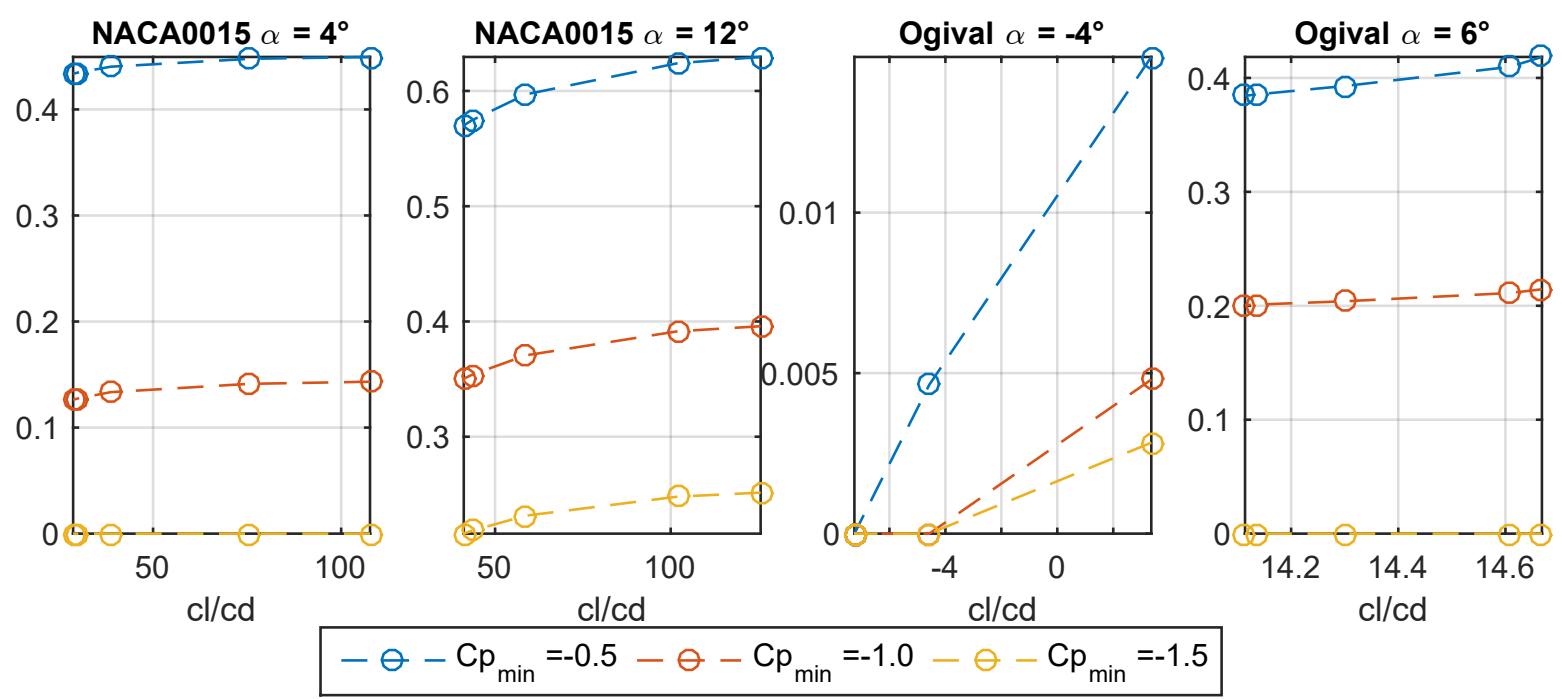

Source: Author

There is a trade-off in increasing lift-to-drag ratio with hydrophobic effect since a more substantial fraction of foil chord is submitted to higher suction pressure.

\subsection{D Propeller Comparatives}

This section aims to compare the performance of propellers without hydrophobicity, previously studied in Sec. 6, with the presence of hydrophobicity, with the slip length values of the two-dimensional analysis.

The simulations are carried out with a rotational speed of 1600 RPM. Six cases of the advance ratio are simulated. The turbulence values are the same as the $2 \mathrm{D}$ simulations.

Two groups of mesh/solver are considered: using the coupled solver with coarse polyhedral mesh; and using segregated solver with hexahedron mesh. The motivation and details of each mesh are explained in Sec. 6.3.4.

\subsubsection{Results: Open Water Diagram}

Figure 46 shows the open water diagram, with the thrust, torque, and the propeller's efficiency for several slip lengths.

It was observed a gain in efficiency, especially for values above $b=1 \times 10^{-6} \mathrm{~m}$ and not significant gains for values above $b=1 \times 10^{-4} \mathrm{~m}$. These results are because of the increase in $K Q$ that, unlike $c d$ in foils, has a large contribution from pressure. 
Figure 46 - Open water diagram with hydrophobic painting and comparison between optimization mesh (polyhedral) and hr2 (hexahedron)

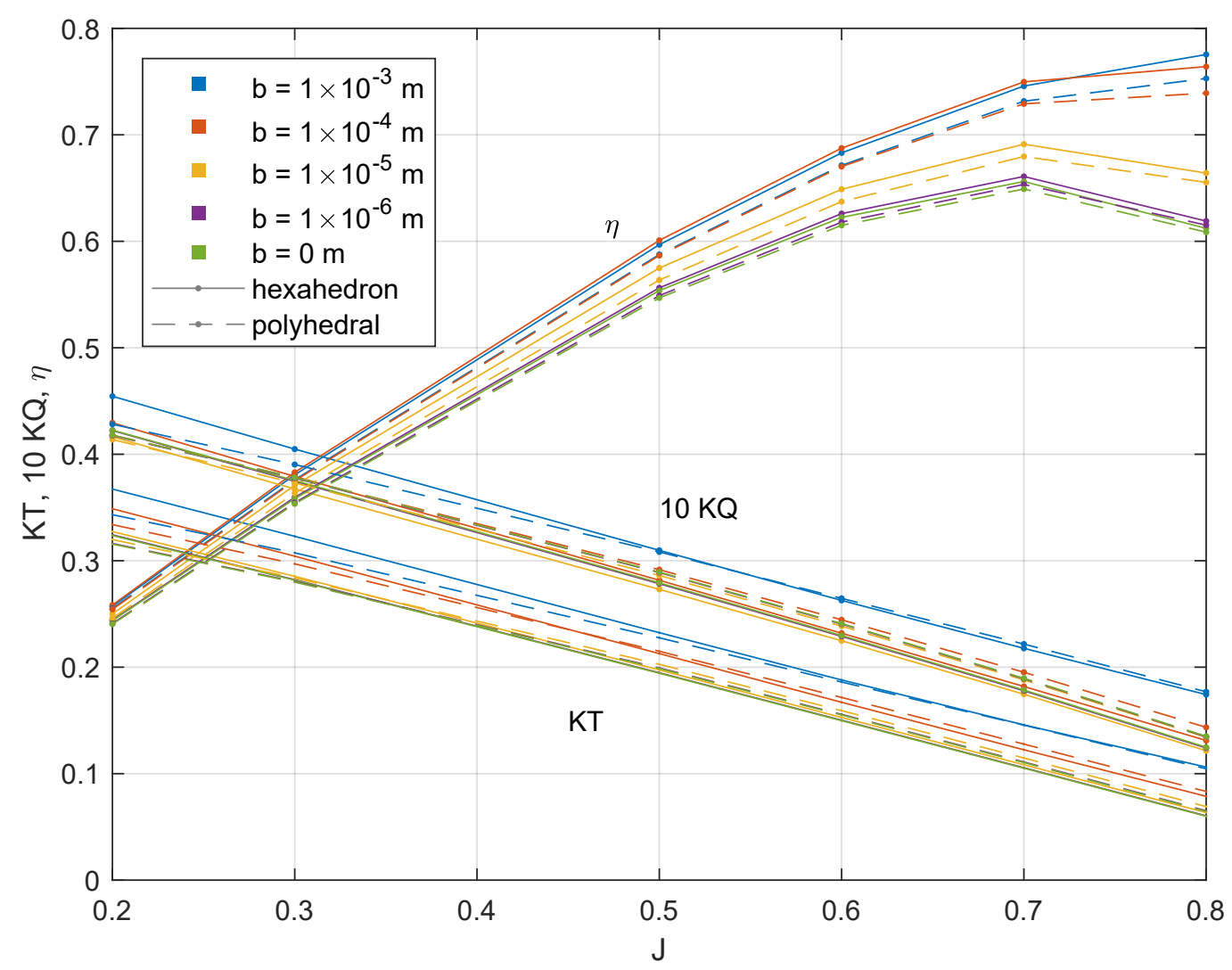

Source: Author

Two operating conditions were analyzed in more detail: low $(J=0.3)$ and high $(J=0.7)$ advance ratio.

\subsubsection{Skin Friction Coefficient}

Figure 47 shows the line integral convolution of skin friction coefficient on the suction and pressure side for $J=0.3$ and Fig. 48, for $J=0.7$. An increase in suction is noted as the hydrophobicity increases, especially in the upper region of the propeller.

\subsubsection{Pressure Coefficient}

Figure 49 shows the pressure coefficient distribution on the suction and pressure side for $J=0.3$. An increase in suction may be noted as the effect of hydrophobicity increases, especially in the upper region of the propeller.

Figure 49 shows the pressure coefficient distribution for $J=0.7$. It is noted an increase in suction and pressure in regions close to the trailing edge. 
Figure 47 - Skin-friction coefficient, varying slip length $b$, for $J=0.3$

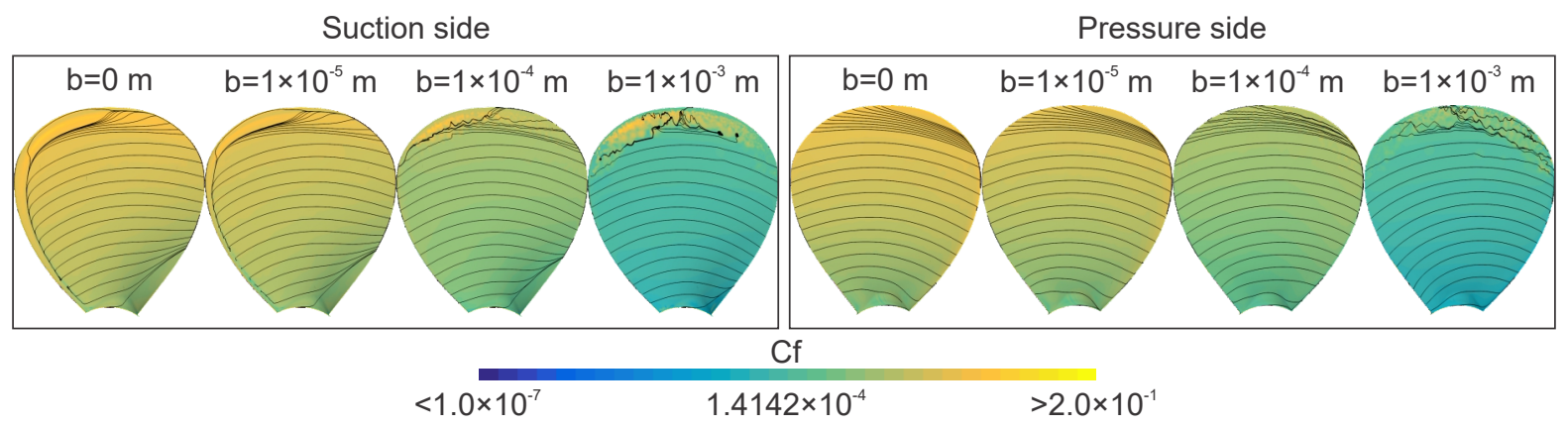

Source: Author

Figure 48 - Skin-friction coefficient, varying slip length $b$, for $J=0.7$

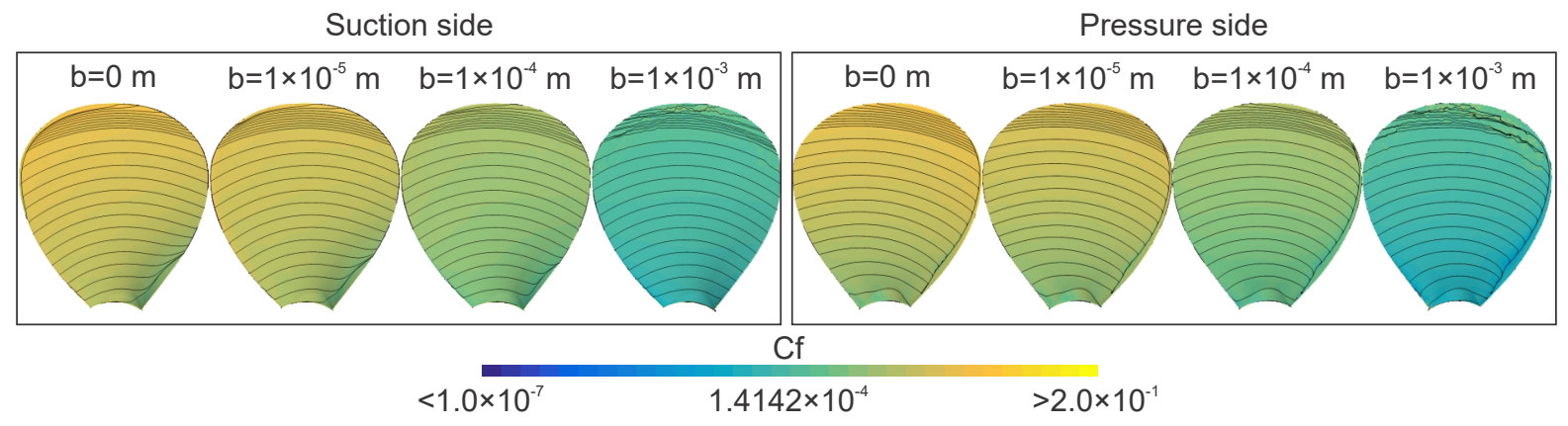

Source: Author

Figure 49 - Coeffient of pressure, varying slip length $b$, for $J=0.3$
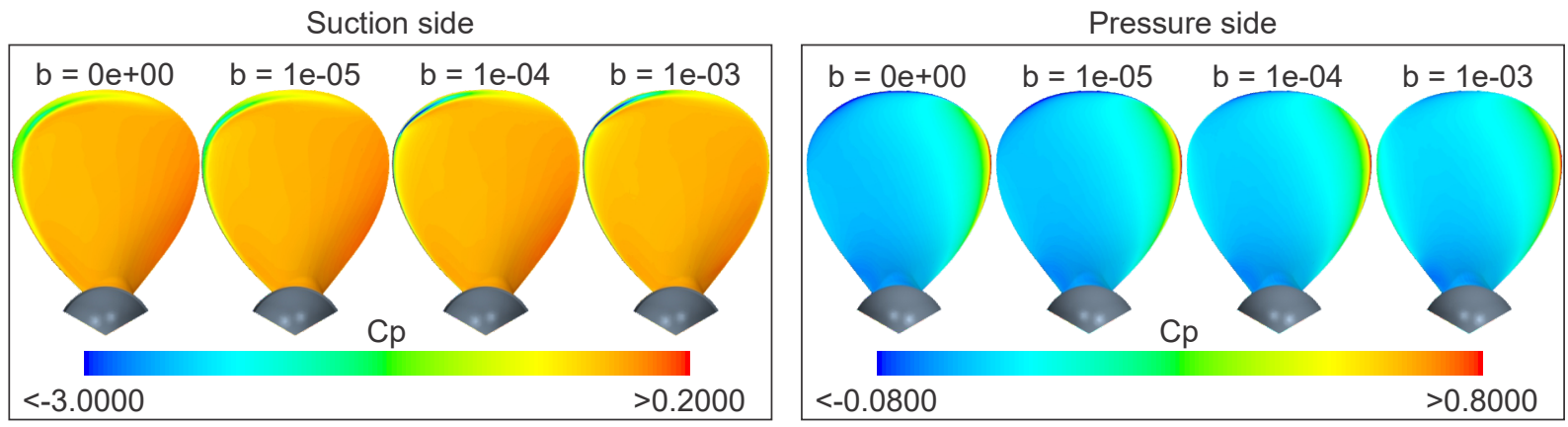

Source: Author

\subsubsection{Segregated and Coupled Solvers}

The results in the Open Water Diagram show that there are some differences in values when using the coarse polyhedral mesh with the coupled solver, which is designed to be fast. For a further investigation, Fig. 51 shows the $C p$ topology and Fig. 52 shows the $C f$ topology. 
Figure 50 - Coeffient of pressure, varying slip length $b$, for $J=0.7$
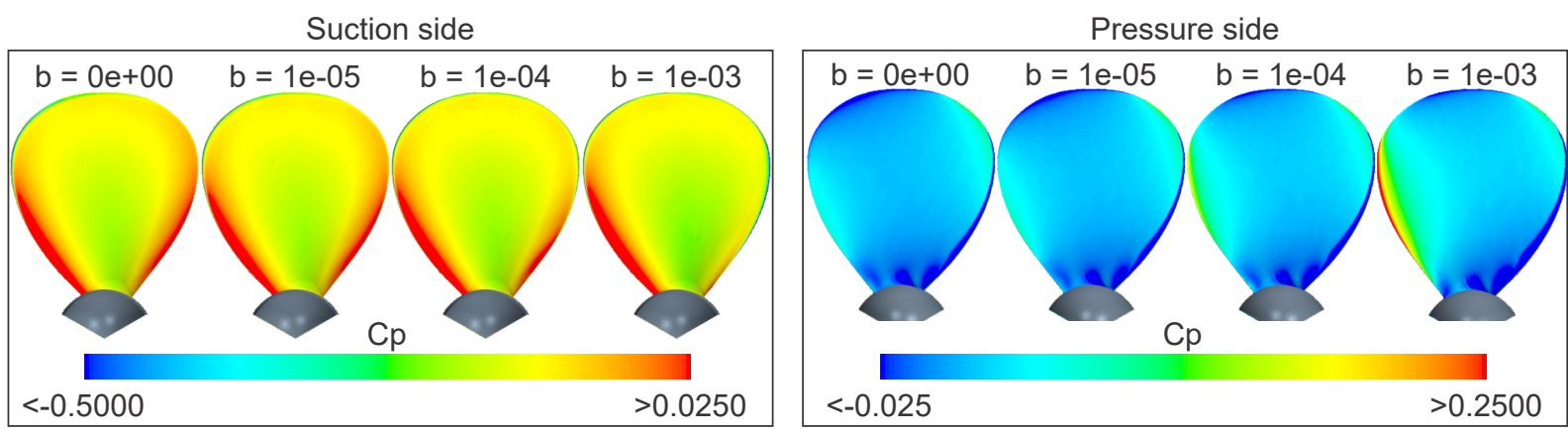

Source: Author

Figure 51 - Comparative of $C p$ between coarse and finer mesh
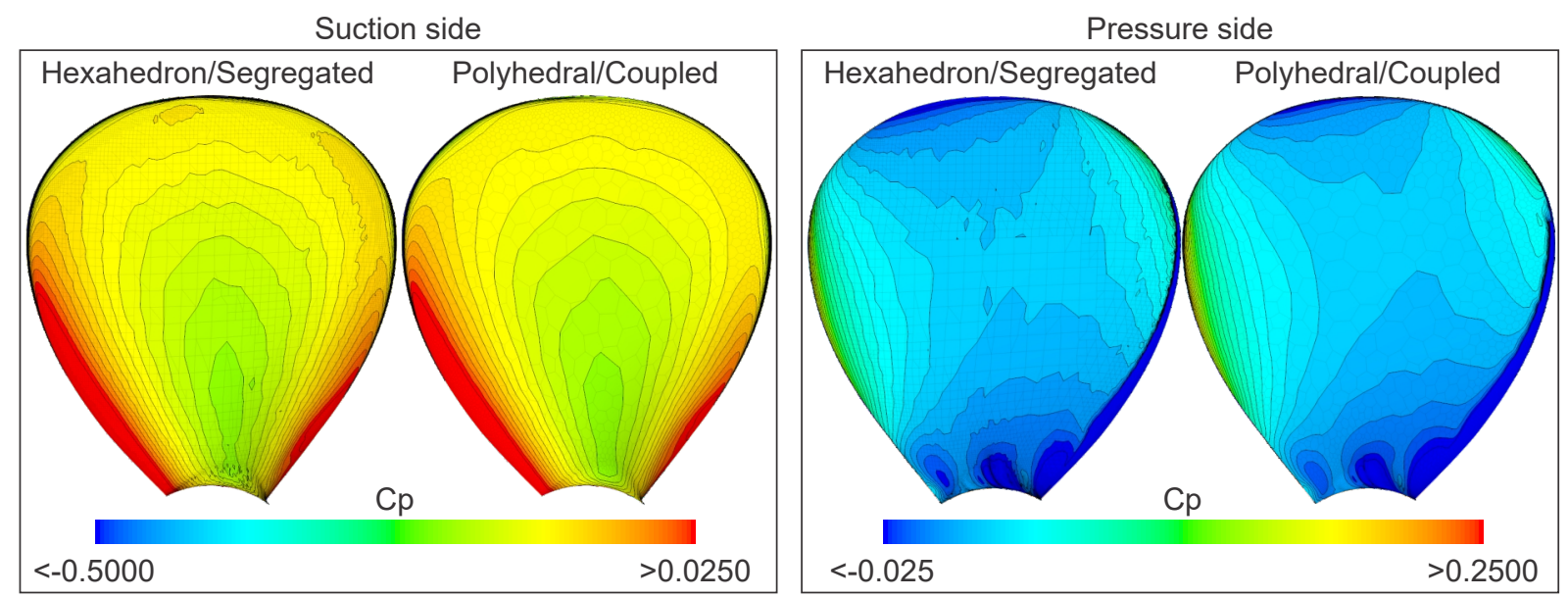

Source: Author

Although it produces different values of hydrodynamic coefficients, the topology is roughly similar. In the case of $C f$, it can be noted that small details of the $C f$ highlighted in Fig. 52 are found in both meshes.

It is concluded that obtaining hydrodynamic coefficients using the polyhedral mesh is not recommended. However, using the optimization framework is acceptable since the topology of $C p$, and $C f$ are reasonably similar, so it should lead to a similarly optimized design. Subsequently, the topology of SHS distribution must be mapped in the hexahedron mesh in order to obtain the hydrodynamic coefficients and allow a fair comparison with other conditions. 
Figure 52 - Comparative of $C f$ between coarse and finer mesh

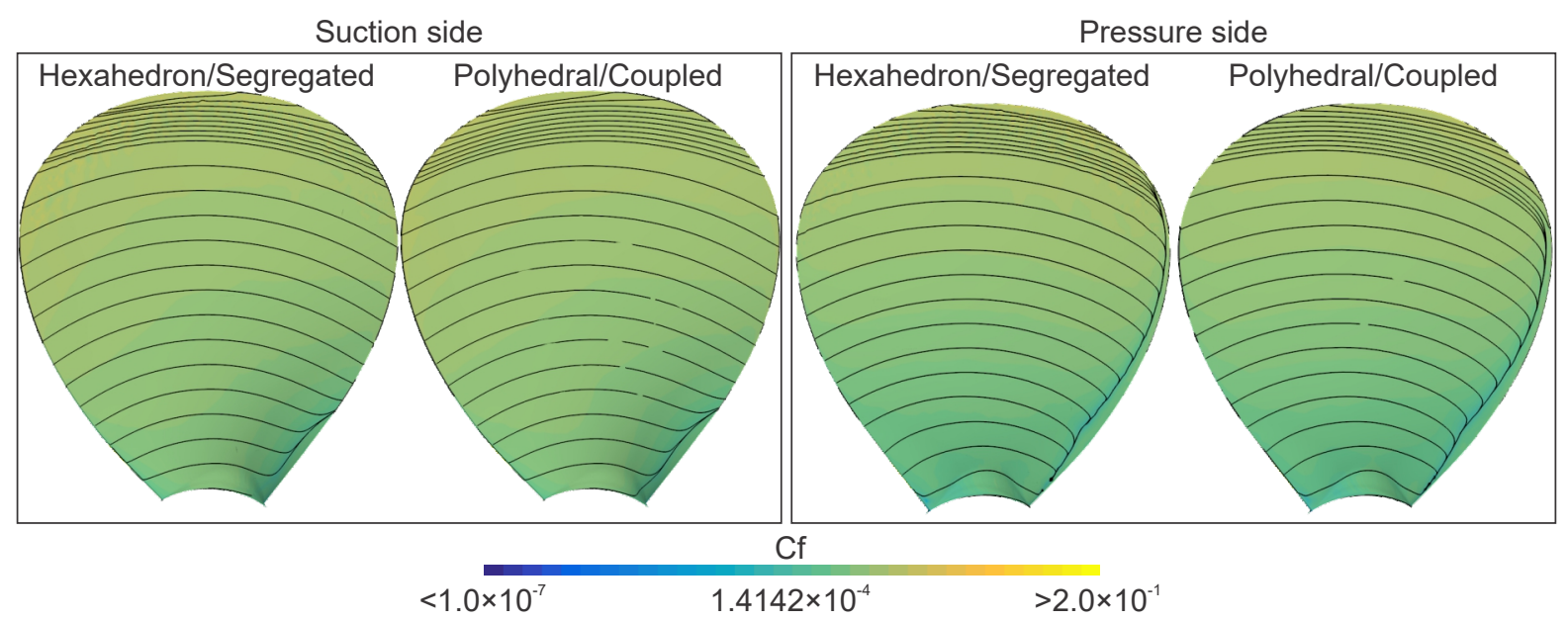

Source: Author

\subsection{Propeller efficiency - $C p$ trade-off}

It can be observed from Fig. 46 that efficiency does not appear to significant increase from $b=1 \times 10^{-4} \mathrm{~m}$ to $b=1 \times 10^{-3} \mathrm{~m}$. However, observing Fig. 49 and Fig. 50, there is an increase in the suction pressure for these values of $b$.

In order to observe the effect of hydrophobicity on the increase of propeller efficiency and in the decrease of pressure (or increase of suction pressure), which can be associated with the cavitation phenomena, an analysis with intermediate values of $b$ are performed $(b=$ $\left.\left[1 \times 10^{-6}, 1 \times 10^{-5}, 8 \times 10^{-5}, 1 \times 10^{-4}, 2 \times 10^{-4}, 4 \times 10^{-4}, 6 \times 10^{-4}, 8 \times 10^{-4}, 1 \times 10^{-3}\right] \mathrm{m}\right)$. The values are compared in terms of propeller efficiency and surface fraction of blade suction-side that presents $C p$ less than -0.2 . Two advance ratio are studied, $J=0.3$ and $J=0.7$. The results are shown in Fig. 53 .

The surface fraction of low $C p$ can indicate regions of possible cavitation presence. It can be noted that in both $J$ there is not a strictly increase relationship of propeller efficiency and area of $C p$, even for $J=0.7$, in which the fraction was below $10 \%$. Therefore, with the assumptions made for modeling the SHS over a propeller, it can conclude with the preliminary results that high values of $b$ are not recommended, once there is a limit of efficiency gain and higher values induce higher surface fraction of low $C p$.

It should be emphasized that these simulations are not performed by using a cavitation model. Since cavitation may affect the effectiveness of hydrophobicity, because this will modify the pressure distribution due to appearance of cavitation bubbles, values of propeller efficiency should be only a guide for a expected behavior of $C p$ since in these simulations propeller efficiency and area of low $C p$ are treated as decoupled, which is not the case when cavitation occurs. 
Figure 53 - Fraction of suction side with $C p<-0.2$ in function of $\eta$ for several values of $b$; and scalar figures of suction face
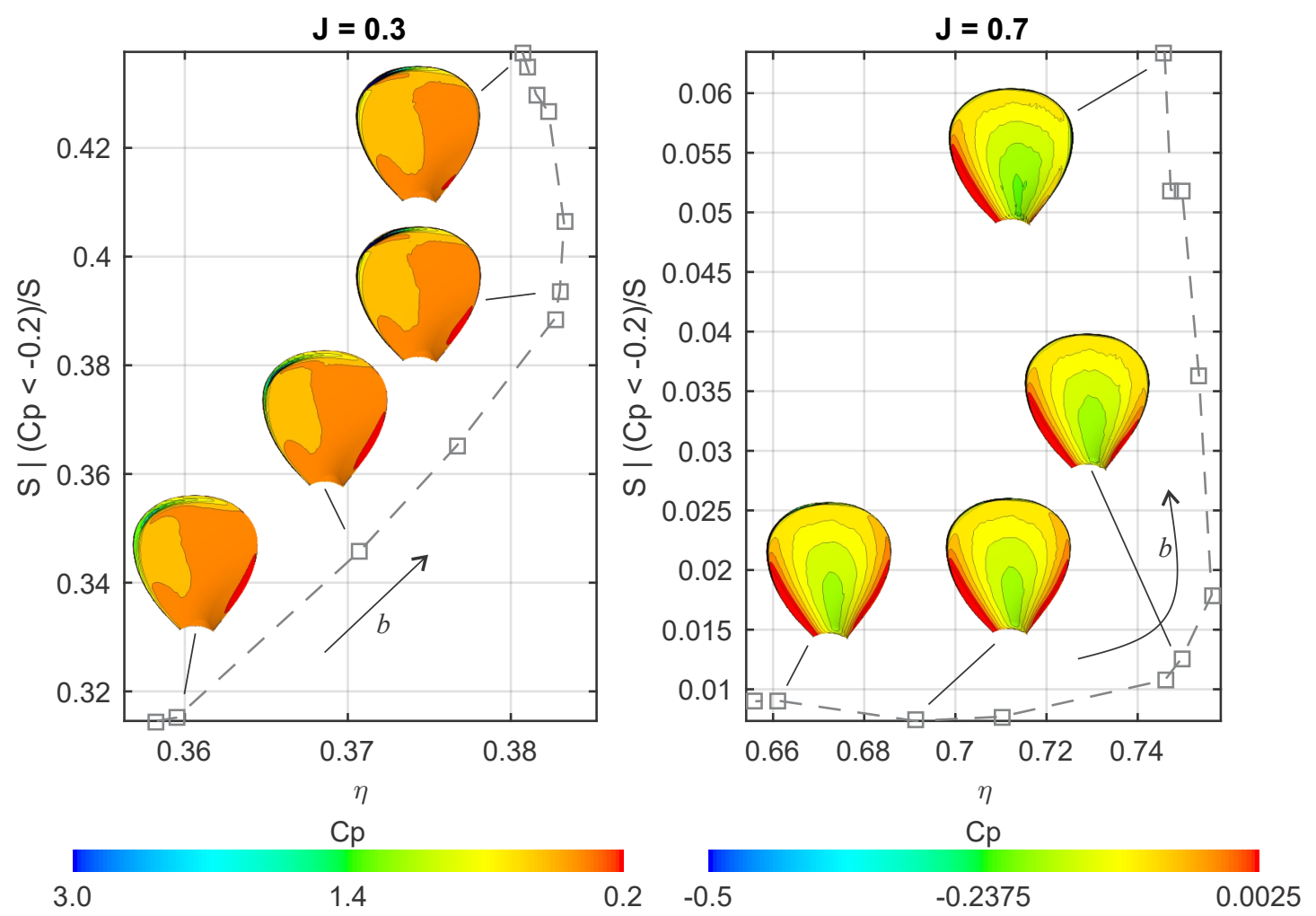

Source: Author

\subsection{Discussions}

It is shown the application of an SHS to the marine propeller for 2D and 3D cases. In the case of 2D simulations, it is obtained satisfactory results, with a considerable drop in drag as higher the hydrophobicity factor. With an increase of hydrophobicity, assessed by increasing slip length, the lift-to-drag ratio is increased. However, it has been observed that this increase in the lift-to-drag ratio is followed by an increase in suction pressure, which can be detrimental for cavitation concern.

Results with propeller show that an increase in slip length is not always followed by an increase in efficiency, presenting an efficiency gain limit. From this limit, the efficiency no longer increases with hydrophobicity, but its area of low pressure continues to grow. For cases studied in this chapter and under the assumptions made before, it can conclude that high values of $b$ are not recommended.

The results motivated the approach to apply the TOM to determine regions of the propeller surface that must turn to an SHS to improve a given objective function and its limits. 


\section{Topology Optimization Results: Two- dimensional cases}

Since the computational cost of performing 3D topology optimization on the propeller's surface is computationally expensive, this chapter aims to be a preliminary step: perform studies in the topology optimization in 2D simulations. Thus, this section has the objective of optimizing the pattern distribution of paint with $\mathrm{SH}$ properties on the surface of a 2D geometry by using the TOM. Two cases, internal and external flow, are presented as the test cases with existing defined geometry, in order to increase its hydrodynamic efficiency. The external flow case is the hydrofoil NACA0015, which was presented previously in Sec. 7.1. For the internal flow case, it is chosen the stretched-S pipe. Once this case was not presented before, Sec. 8.1 shows the geometry and adopted boundary conditions. As 2D simulations are relatively fast, this chapter analyzes some parameters that define the optimization problem, such as the influence of surface constraint, slip length, Reynolds number, and angle of attack, in Sec. 8.3. Before, some analyses are made to check if sensitivity for the hydrophobic problem is returning expected values in Sec. 8.2.

\subsection{Internal flow test case}

The test case selected to represent an internal flow is a stretched-S pipe. It represents a pipe in a turbulent flow regime with two soft-corners. The geometry and mesh topology are shown in Fig. 54 with the adopted boundary conditions and the design surface.

Figure 54 - Domain dimensions and boundary conditions for stretched-S pipe - internal flow case. Dimensions in $m$ (left); and mesh topology with 13721 elements and 482 design elements on walls (right)

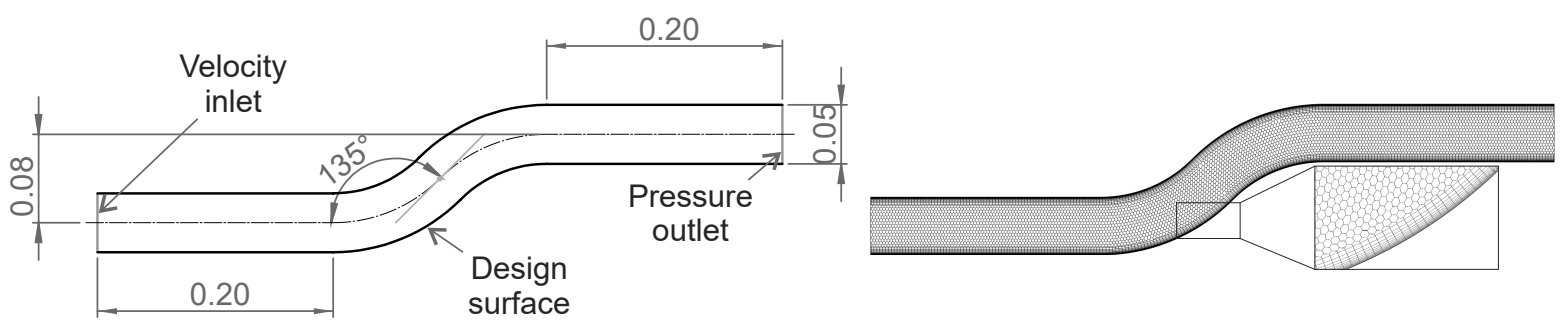

Source: Author

For the internal case, the pipe width is the characteristic length $\ell$. The target Reynolds is $R e=1.0 \times 10^{5}$. Therefore, the maximum velocity on the inlet is $v_{a}=1.78 \mathrm{~m} / \mathrm{s}$, and the boundary condition is given by a velocity profile from Prandtl's one-seventh-power 
law. Turbulence intensity is defined on the inlet as $5 \%$. The characteristic turbulent length adopted is $7 \%$ of the characteristic length.

\subsection{Sensitives comparison: Adjoint and Finite Difference Methods}

Obtaining sensitivity of hydrophobic influence using adjoint methods or, even though, getting a 2D sensitivity (blade surface) from the 3D problem (propeller) (or, for the two-dimensional cases, 1D sensitivity from the 2D problem) is a novel application that the hypotheses are developed in Sec. 4. This section aims to compare with FDM and verify if the values obtained through adjoint methods are similar. FDM is a flexible approach to obtain the sensitivity once it treats the problem as a black-box, but its computational cost makes it unfeasible to calculate several times for an optimization framework.

The study case is NACA0015 foil with $\hat{\rho}=0.5$ in all surfaces, using a slip length of $b=1 \times 10^{-4} \mathrm{~m}$, angle of attack $\alpha=8.0^{\circ}$ and $C=c d$. To obtain the sensitivity by FDM, it uses 4 points and adopting $\Delta \hat{\rho}=0.025$ by changing only one position for each point. Remaining pseudo-density are maintained as 0.5. The formulation to obtain from FDM is:

$$
\begin{aligned}
\left.\frac{d \phi}{d \hat{\rho}}\right|_{\mathrm{FDM}}=\frac{1}{\Delta \hat{\rho}}[ & \frac{1}{12} \phi\left(\hat{\rho}_{i}+2 \Delta \hat{\rho}\right)-\frac{2}{3} \phi\left(\hat{\rho}_{i}+\Delta \hat{\rho}\right)+ \\
+ & \left.\frac{2}{3} \phi\left(\hat{\rho}_{i}-\Delta \hat{\rho}\right)-\frac{1}{12} \phi\left(\hat{\rho}_{i}-2 \Delta \hat{\rho}\right)\right]
\end{aligned}
$$

Figure 55 shows the comparison between each method. Both upper and lower foil surfaces are plotted. There are some noisy results close to the leading edge, but the mean value is similar to the values obtained using the adjoint method.

The results are considered satisfactory, and the formulations developed in Sec. 4 are adopted for sensitivity calculations of all SHS problems. 
Figure 55 - Comparison between sensitivity obtained using Adjoint and Finite Difference Methods

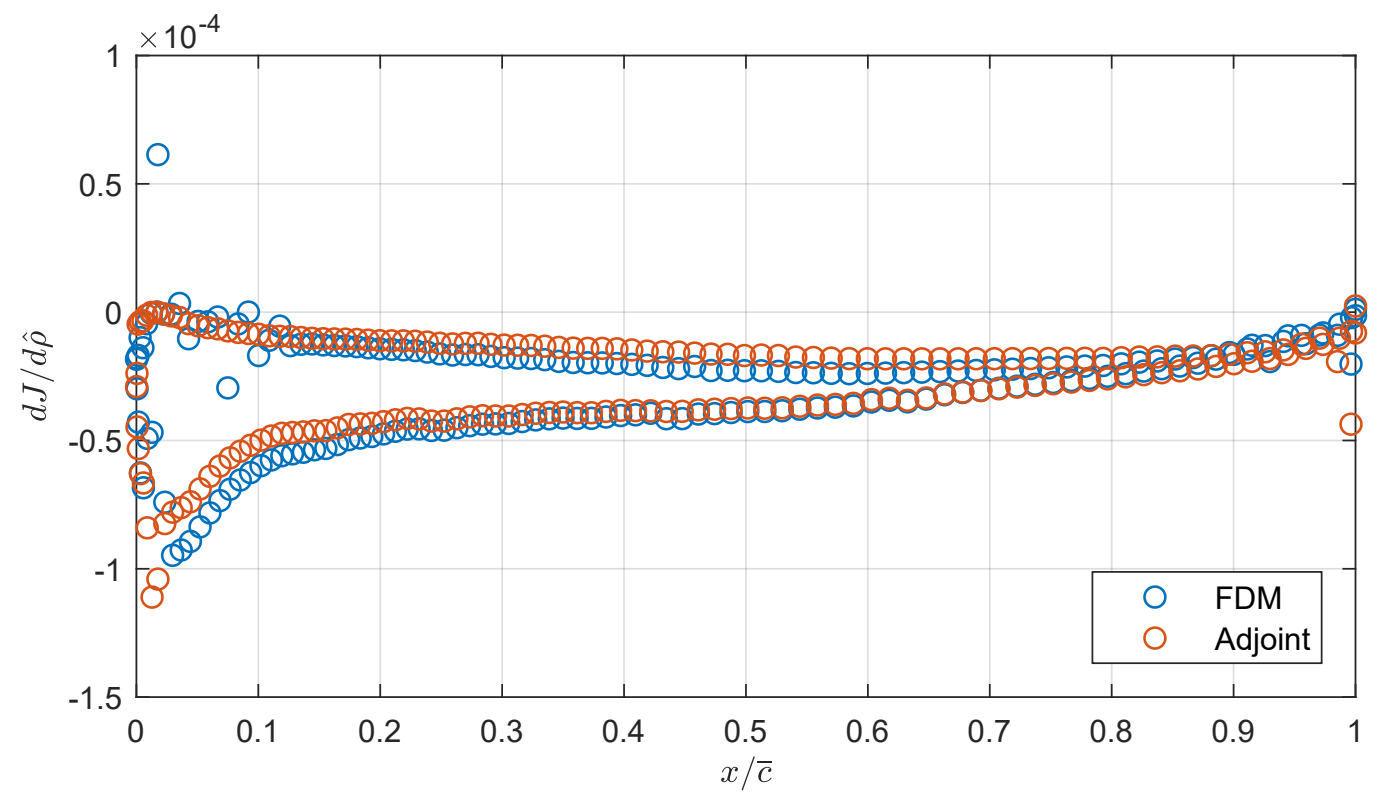

Source: Author

\subsection{Results of Topology Optimization}

\subsubsection{Comparison with proposed topologies}

This section aims to compare a suggested distribution of an SHS based only on a guessed intuition, without using any optimization procedure, with the result obtained by using Topology Optimization. For this study, half of a NACA0015 foil surface $(\hat{g}=0.5)$ is a super-hydrophobic surface with slip length of $b=1 \times 10^{-4} \mathrm{~m}$. The simulated angle of attack $\alpha$ is $8^{\circ}$. The optimization formulation, presented in Eq. (3.3), has a cost function to minimize the drag coefficient restricted by a maximum surface $\hat{g}^{*}$. The optimization formulation is presented in Eq. (8.2).

$$
\begin{array}{|ll}
\operatorname{Min}_{\hat{\rho}} & C=c_{d}=\frac{2}{\rho v_{a}^{2} c} D \\
\text { s.t. } & \hat{g}(\hat{\rho})=\frac{\int_{\Theta} \hat{\rho} d A}{\int_{\Theta} d A} \leq \hat{g}^{*} \\
& 0 \leq \hat{\rho} \leq 1
\end{array}
$$

Three proposals of guessed-intuitive based ideas, (i.e., without any optimization procedure) for SHS distribution on half of the foil surface are presented in Fig. 56.

One proposal (Upper) is based on covering only the upper surface since most of the lift and friction are on this side. Another proposal (LE) is to cover the leading edge because the peaks of velocity and skin friction are closer to the leading edge. Finally, the 
Figure 56 - Proposals for SHS distributions with $\hat{g}=0.5$. Highlighted contours indicate presence of SHS: (i) Upper; (ii) LE (Leading Edge); and (iii) $C f$ based

(i)

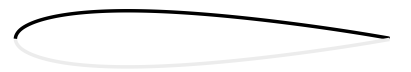

(ii)

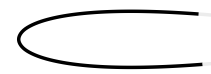

Pseudo-density - $\hat{\rho}$

$\begin{array}{llc}\text { (No Paint) } & 1.0 \\ & 0.5 & \text { (SHS) }\end{array}$

Source: Author

last proposal $\left(C_{f}\right.$ - based $)$ is based on $C_{f}$ distribution, as shown in Fig. 57 . It can also be noted that $y^{+}$is below 1.0 over the foil. Along the chord, the face covered is the one that has the highest $C_{f}$. Therefore, the coverage is reversed after the position $x / c=0.4$, being on the lower surface.

Figure 57 - Skin Friction coefficient (left) and wall $y^{+}$(right) distributions
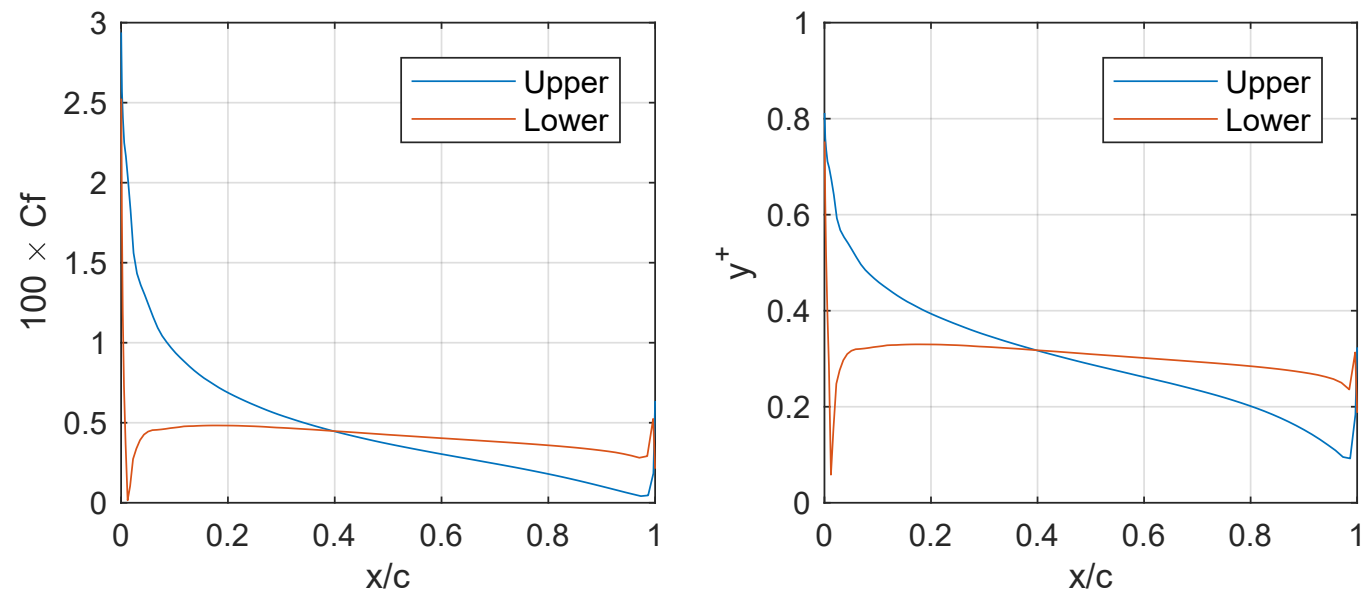

Source: Author

Despite the proposals, the result from topology optimization, shown in Fig. 58, is none of the proposals. The comparison between the value of the cost function is shown in Fig. 59. Indeed, the obtained distribution from topology optimization, although with the unexpected small strip of SHS in the bottom of the foil, is the one with the lowest drag.

Figure 58 - SHS distribution resulted from Topology Optimization

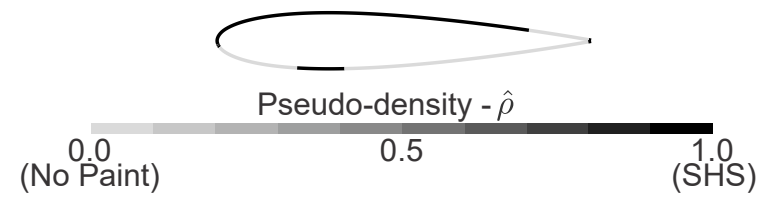

Source: Author

These results endorse the use of topology optimization, which can find unexpected results and noncontinuous distributions, i.e., it is possible to have several parts with SHS, not only a single continuous path. 
Figure 59 - Comparison of $c_{d}$ for NACA0015: $R e=1.05 \times 10^{6}, \alpha=8^{\circ}, \bar{b}=1 \times 10^{-4} \mathrm{~m}$, and $\hat{g}^{*}=0.5$

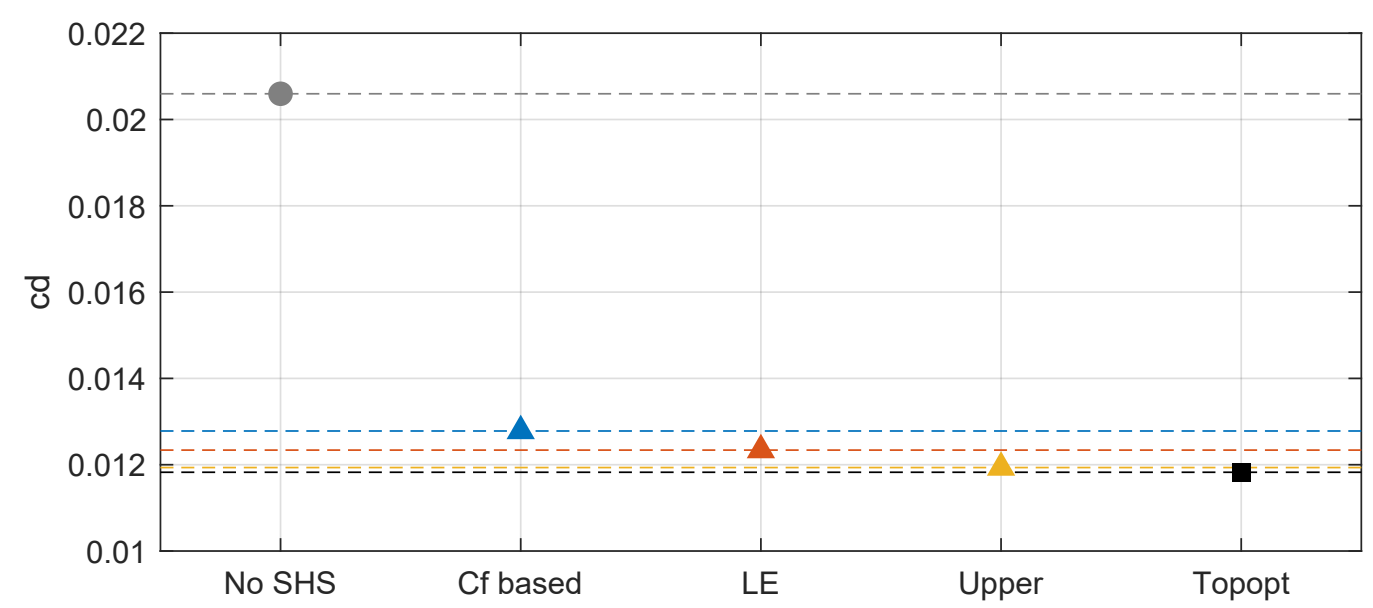

Source: Author

Figure 60 - Comparison of $c d$ (left) and the obtained topology (right) for NACA0015 varying $\bar{b}: R e=1.05 \times 10^{6}, \alpha=8^{\circ}$, and $\hat{g}^{*}=0.5$

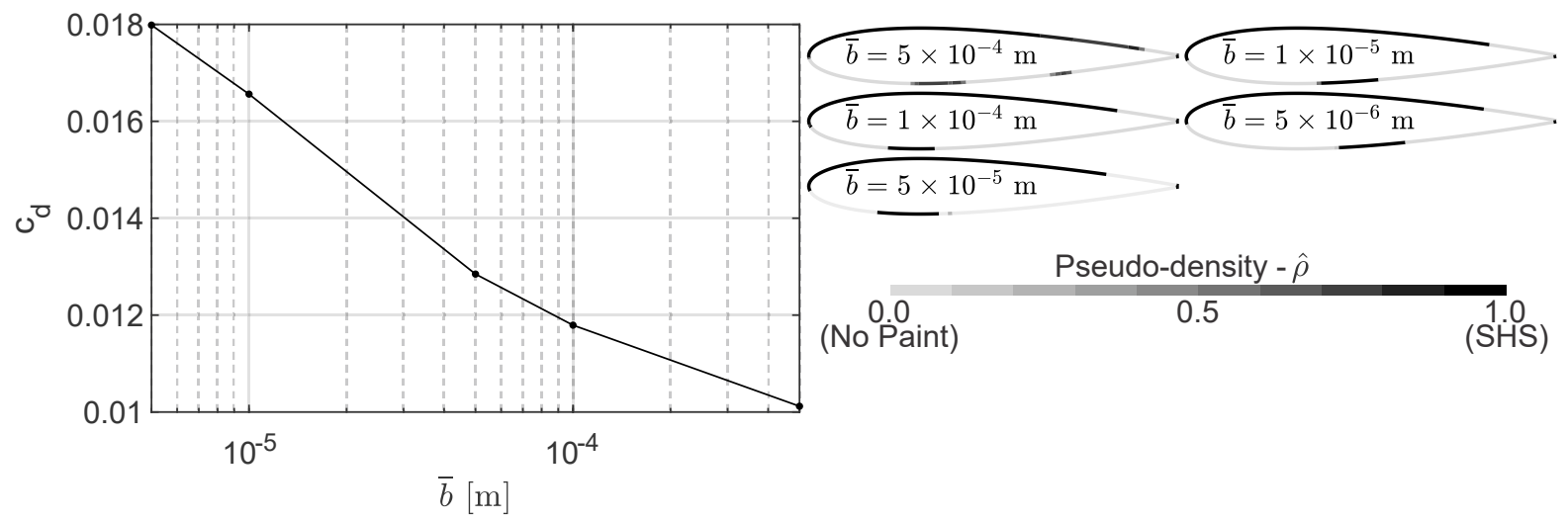

Source: Author

\subsubsection{Influence of slip length}

This subsection aims to check how the slip length, which can be interpreted as the intensity of hydrophobicity, affects the topology of paint distribution. For this study, internal and external flows are evaluated, and, in both cases, all other parameters, such as operating condition and surface constraint, are maintained constant.

For the external flow case, Fig. 60 shows the drag coefficient after the optimization process for five different values of maximum slip length $\bar{b}$, which corresponds to $\hat{\rho}=1.0$, and obtained topology from the optimization process. In all cases, $\hat{g}^{*}=0.5, \alpha=8^{\circ}$, $R e=1.05 \times 10^{6}$ and $q=3.0$.

Figure 61 shows the streamlines for each slip length. Since the topologies are similar for several slip lengths, the streamlines seem to follow a pattern: the higher the slip length, 
the closer they are to the foil. Considering a volume control that contains all domain but the foil: since the foil's (module of) drag is lower as higher the slip length is, it can be interpreted as the foil being a lower sinkage term in the momentum equations as lower is the drag or as higher is the slip length. With this lack of momentum, the streamlines get closer to the foil.

Figure 61 - Streamlines arround NACA0015 foil for several optimized solutions, varying $\bar{b}$ : $R e_{x}=1.05 \times 10^{6}, \bar{b}=1 \times 10^{-4}$ and $\hat{g}=0.5$

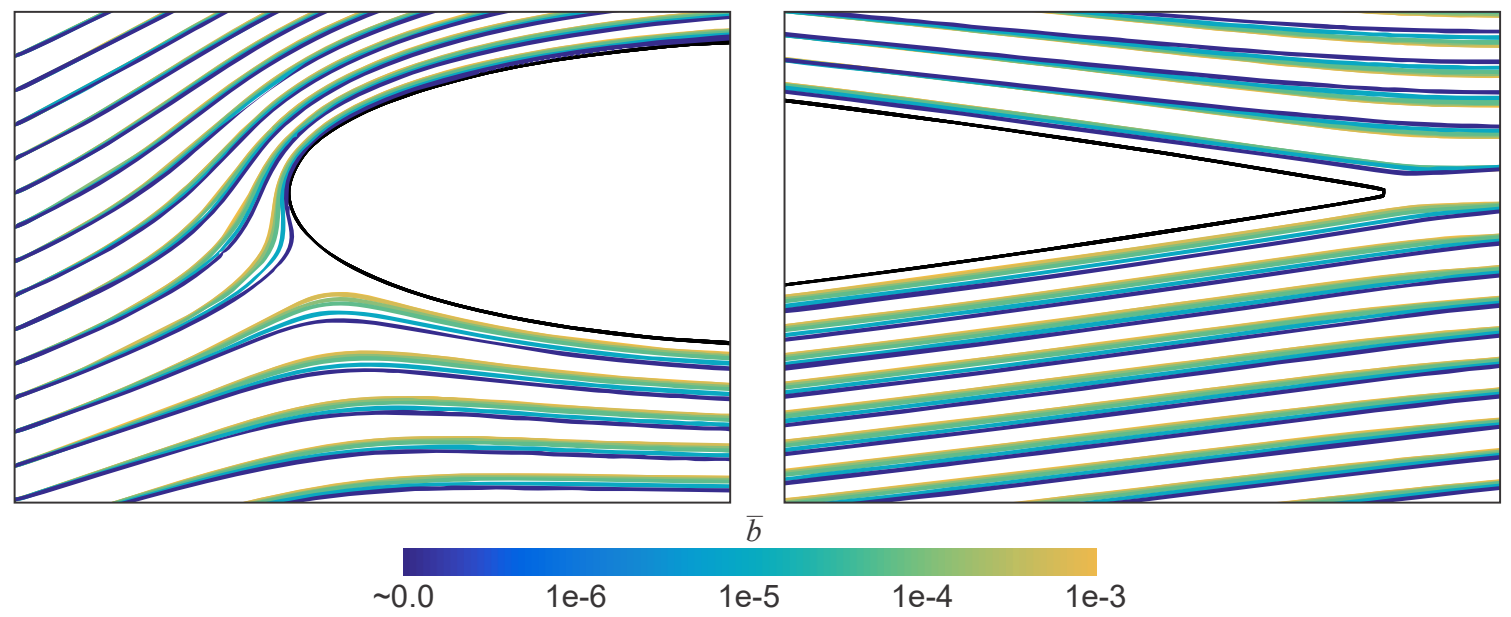

Source: Author

For the internal flow case, Fig. 62 shows the pressure drop after the optimization process for the same batch of maximum slip length $\bar{b}$ and the obtained topology. As the external flow case, the surface constraint limit is $\hat{g}^{*}=0.5$ and all other variables have remained the same: $R e=1 \times 10^{5}$ and $q=1.5$.

In both cases it can be noted that the higher the slip length, the lower the drag or pressure drop. There are, indeed, some differences of topology solutions, notably the condition of $\bar{b} \leq 1 \times 10^{-5} \mathrm{~m}$ for the external flow case. However, the topologies along slip length are considerably similar for all cases: for external flow case, the upper surface is covered more than half and a small section close to the middle is painted on bottom surface; for internal flow case, the observed paint patterns are applied before and on the first curve in the top part and on the second curve in the bottom part.

This is an interesting conclusion because even though not having a precise value of slip length, these results show that the topology optimization solutions seem not to change considerably with the slip length.

\subsubsection{Influence of surface constraint}

This subsection aims to evaluate the obtained topology of paint distribution for several surface constraint levels. Limiting the amount of paint can be extrapolated to a real-case scenario as having a limited budget to the amount of area that can be turned 
Figure 62 - Comparison of $p_{\text {drop }}$ (left) and the obtained topology (right) for stretched-S pipe varying $\bar{b}: R e=1 \times 10^{4}$, and $\hat{g}^{*}=0.5$

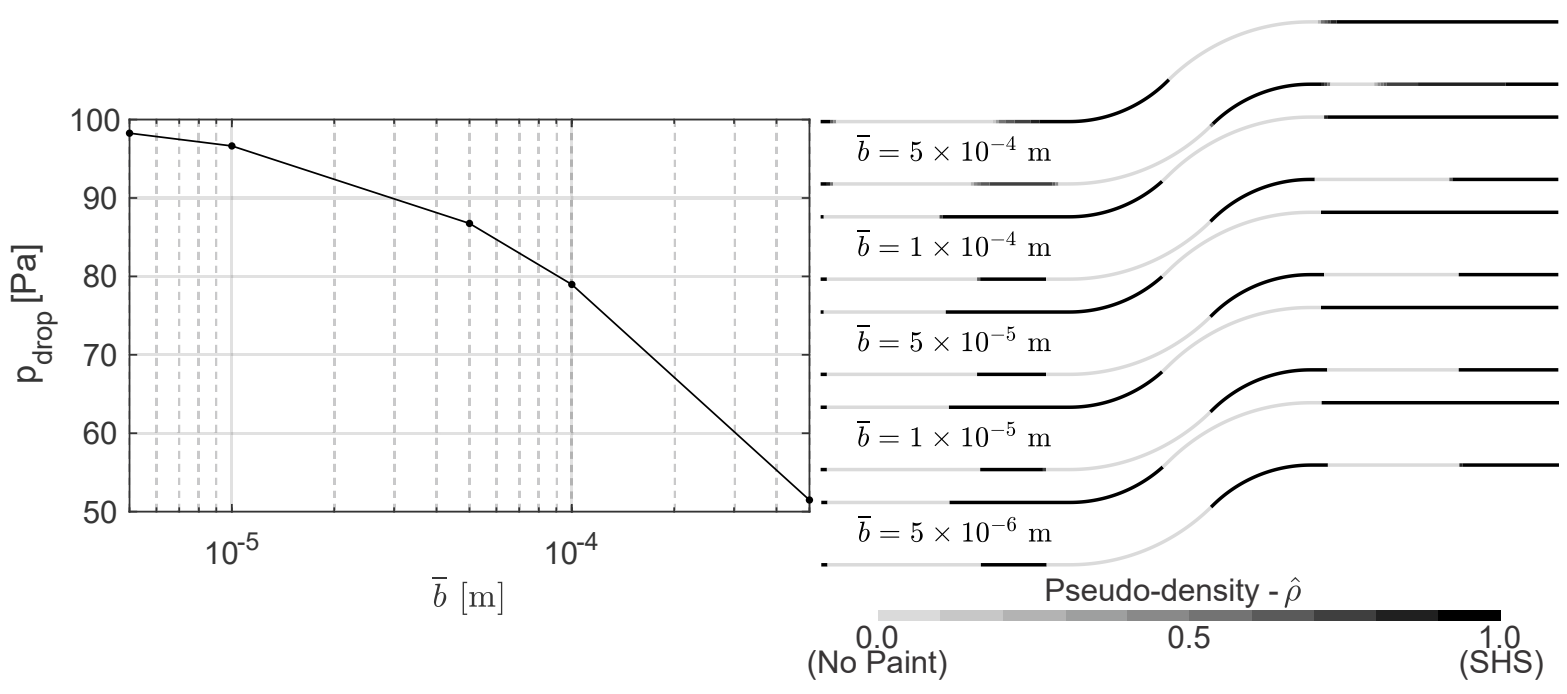

Source: Author

into an SHS. Also, by increasing the limit of surface constraint can show the increase of areas to prioritize in order to have the optimized drag or pressure drop reductions.

For the external flow case, Fig. 63 shows the drag coefficients and the obtained topology for ten different surface constraints $\hat{g}^{*}$. Other parameters, such as the angle of attack, Reynolds number, and slip length are maintained constant. The conditions of foil fully covered by SHS (plotted in $\hat{g}=1$ ) and without SHS (plotted in $\hat{g}=0$ ), despite of not representing optimization results, are also plotted to compare the drag coefficient.

Figure 63 - Comparison of $c_{d}$ (left) and the obtained topology (right) for NACA0015 varying $\hat{g}^{*}: R e=1.05 \times 10^{6}, \alpha=8^{\circ}$, and $\bar{b}=1 \times 10^{-4} \mathrm{~m}$
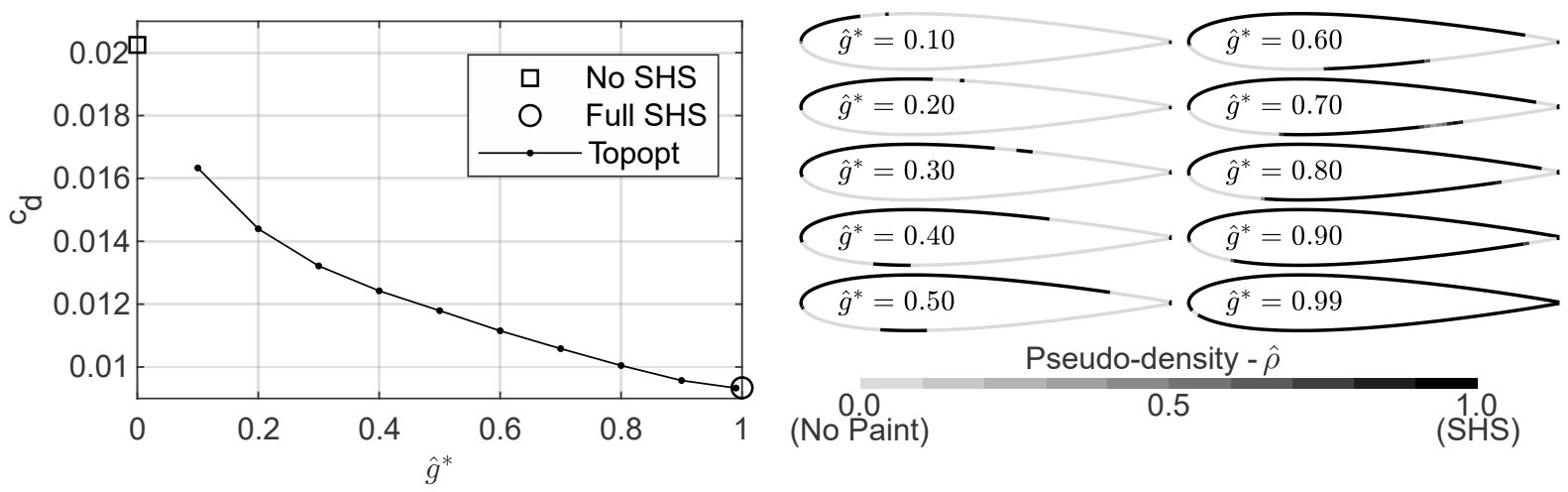

Source: Author

It is interesting to notice that, between $\hat{g}=0.3$ and $\hat{g}=0.4$, the optimized solution started to paint the bottom part. Figure 64 shows the streamlines generated by some constraint (plus the no-SHS and full-SHS conditions), detailing the leading and trailing 
Figure 64 - Comparison of $c d$ for NACA0015, varying $\alpha$ for $R e_{x}=1.05 \times 10^{6}, \bar{b}=1 \times 10^{-4}$ and $\hat{g}=0.5$

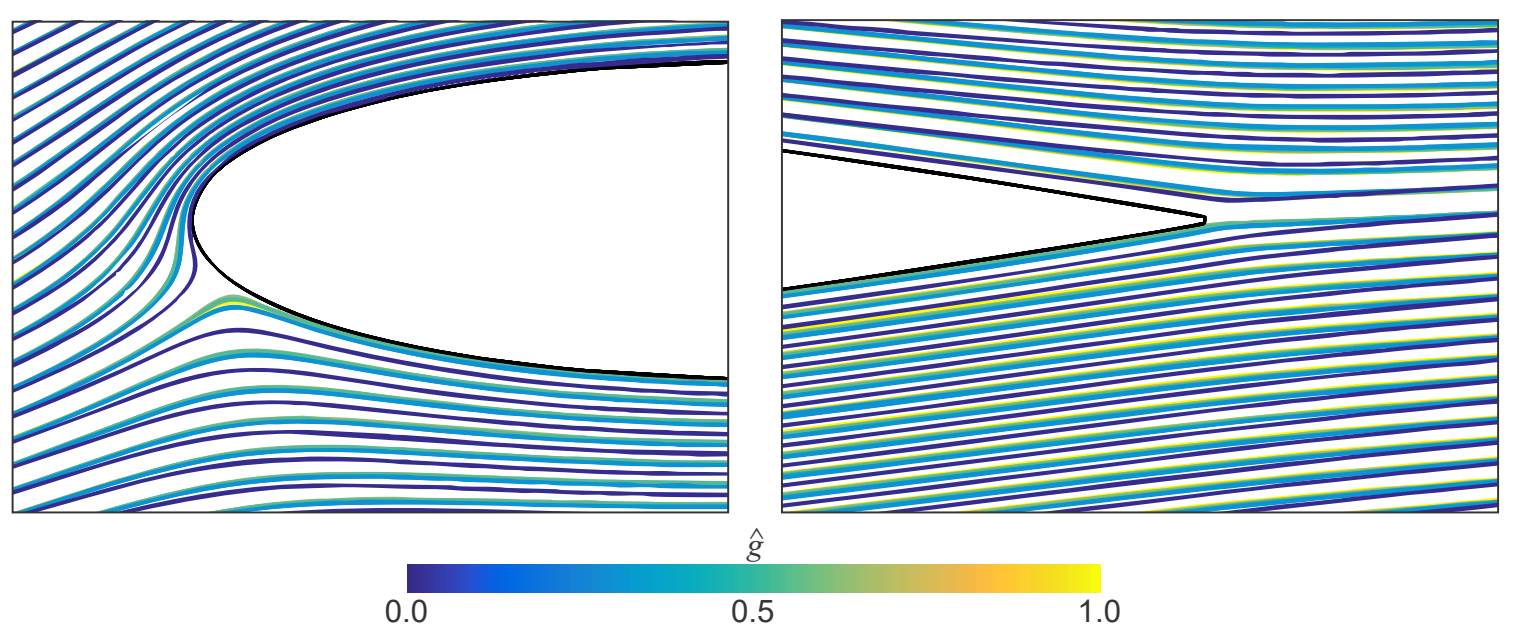

Source: Author

edges.

As the presence of SHS starts on the trailing edge, for lower analyzed $\hat{g}$ it is possible to see the effect on the streamlines, between No-SHS and $\hat{g}=0.3$ conditions. Note, in the leading edge part, that streamlines that present SHS are very close to each other. These results emphasize a consequence seen by the optimization: with a small amount of freedom to distribute the SH painting, one of the first designs focused on to change the velocity field close to the stagnation point and upper surface. This result is expected, since the higher friction zones are in the upper surface, close to the leading edge.

For the internal flow case, Fig. 65 shows the pressure drop for several surface constraint limits $\hat{g}^{*}$. For comparison purposes, the case of No-SHS and the design surface fully covered by SH paint are also presented. When limited by $\hat{g}^{*}=0.99$, it is obtained a pressure drop lower than a fully covered case. Indeed, Fig. 66 shows the topology for each surface constraint limit and it is possible to observe that the topology does not cover $99 \%$ of surface for $\hat{g}^{*}=0.99$.

In order to investigate the surface constraint, Fig. 67 shows the relation of the percentage of surface covered by SHS $\hat{g}$ and the surface constraint limit $\hat{g}^{*}$. For $\hat{g}^{*} \leq 0.9$, it can be observed that the surface covered by SHS is lying in the constraint limits. However, for $\hat{g}^{*}=0.99$, the surface constraint is not active.

The case when the surface constraint is not active is not found in the external flow case. The leading reason can be related to the reverse flow, which is more intense in this case and is not present in the external flow case (at least when simulating in $\alpha=8^{\circ}$ ). Since, for lower Reynolds, it is expected to have higher recirculation zones, the next subsections aim to investigate if the painting distribution is even lower. 
Figure 65 - Comparison of $p_{\text {drop }}$ for stretched-S pipe varying $\hat{g}^{*}: R e=1 \times 10^{5}$, and $\bar{b}=1 \times 10^{-4} \mathrm{~m}$

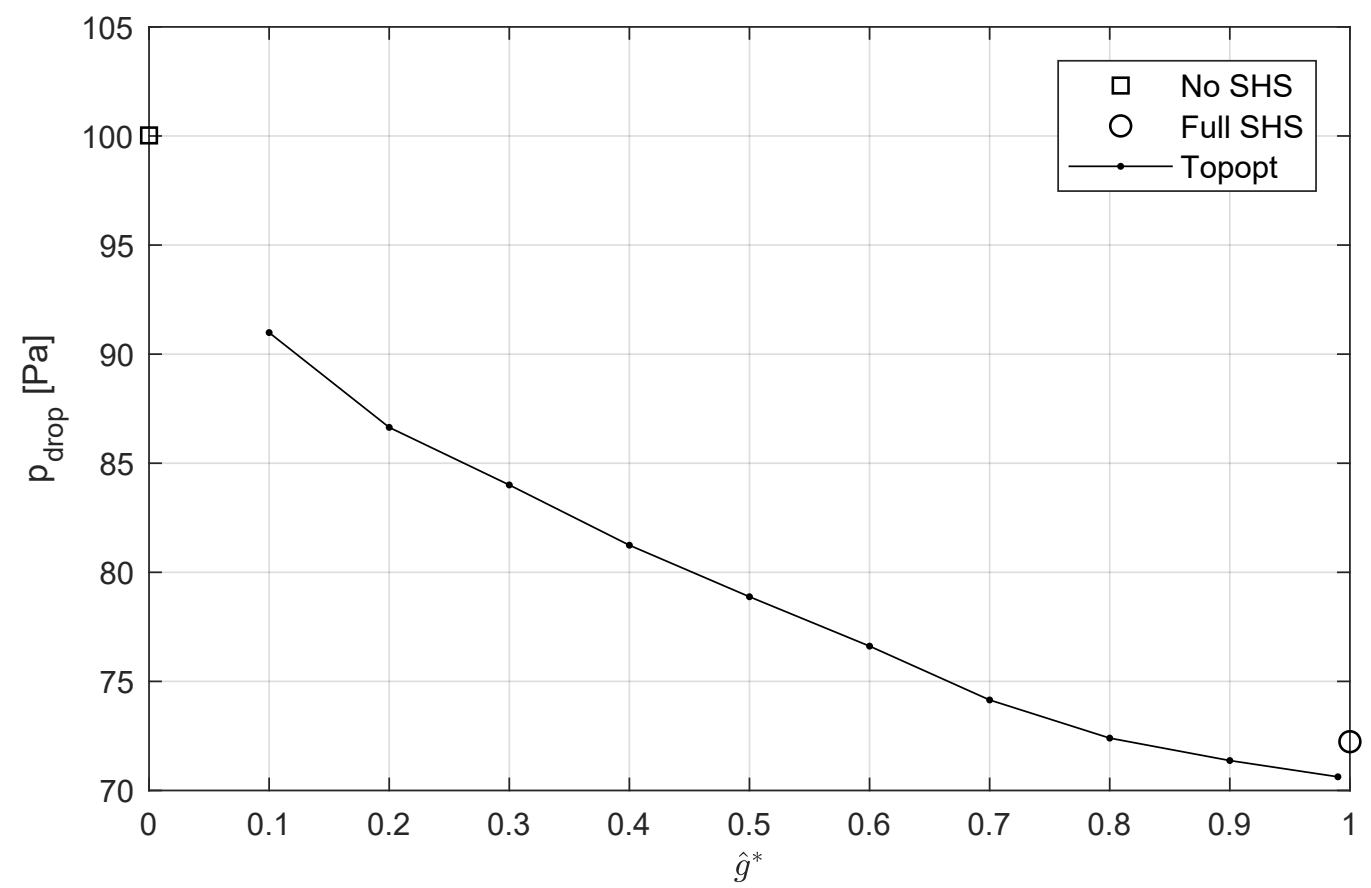

Source: Author

Figure 66 - Comparison of obtained topology for stretched-S pipe varying $\hat{g}^{*}: R e=1 \times 10^{5}$, and $\bar{b}=1 \times 10^{-4} \mathrm{~m}$
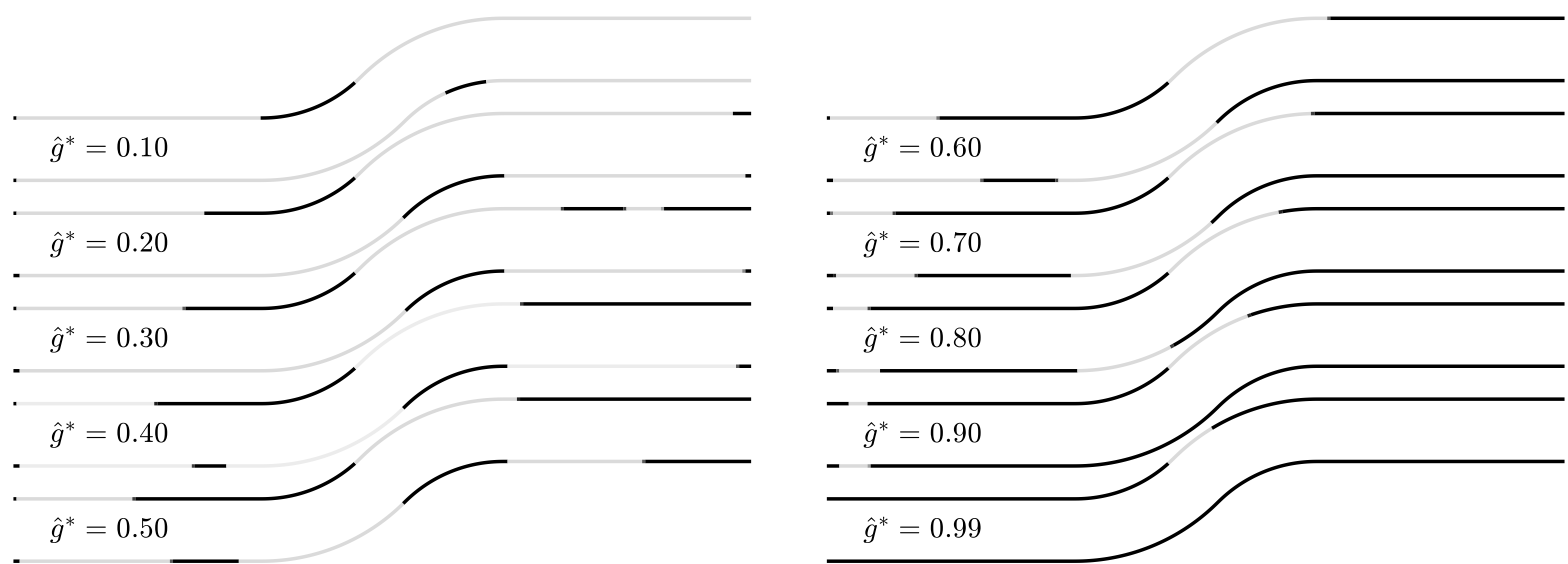

Source: Author

\subsubsection{Influence of Reynolds number}

This subsection aims to check the effect of the Reynolds number in the topology optimization process. To evaluate it, the advance velocity $v_{a}$ is changed, maintaining all other parameters, such as mesh, surface constraint, and slip length, constant.

For the external flow case, Fig. 68 shows that for the analyzed Reynolds numbers, wall $y^{+}$values are still close to 1.0 and, regarding some small points, below 1.0. Figure 69 shows the resulted topology and the drag coefficient, respectively, for several Reynolds 
Figure 67 - Relation of surface constraint limit $\hat{g}^{*}$ and effective percentage of surface covered by SHS $\hat{g}$ for stretched-S (internal flow) case: $R e=1 \times 10^{5}$, and $\bar{b}=1 \times 10^{-4} \mathrm{~m}$

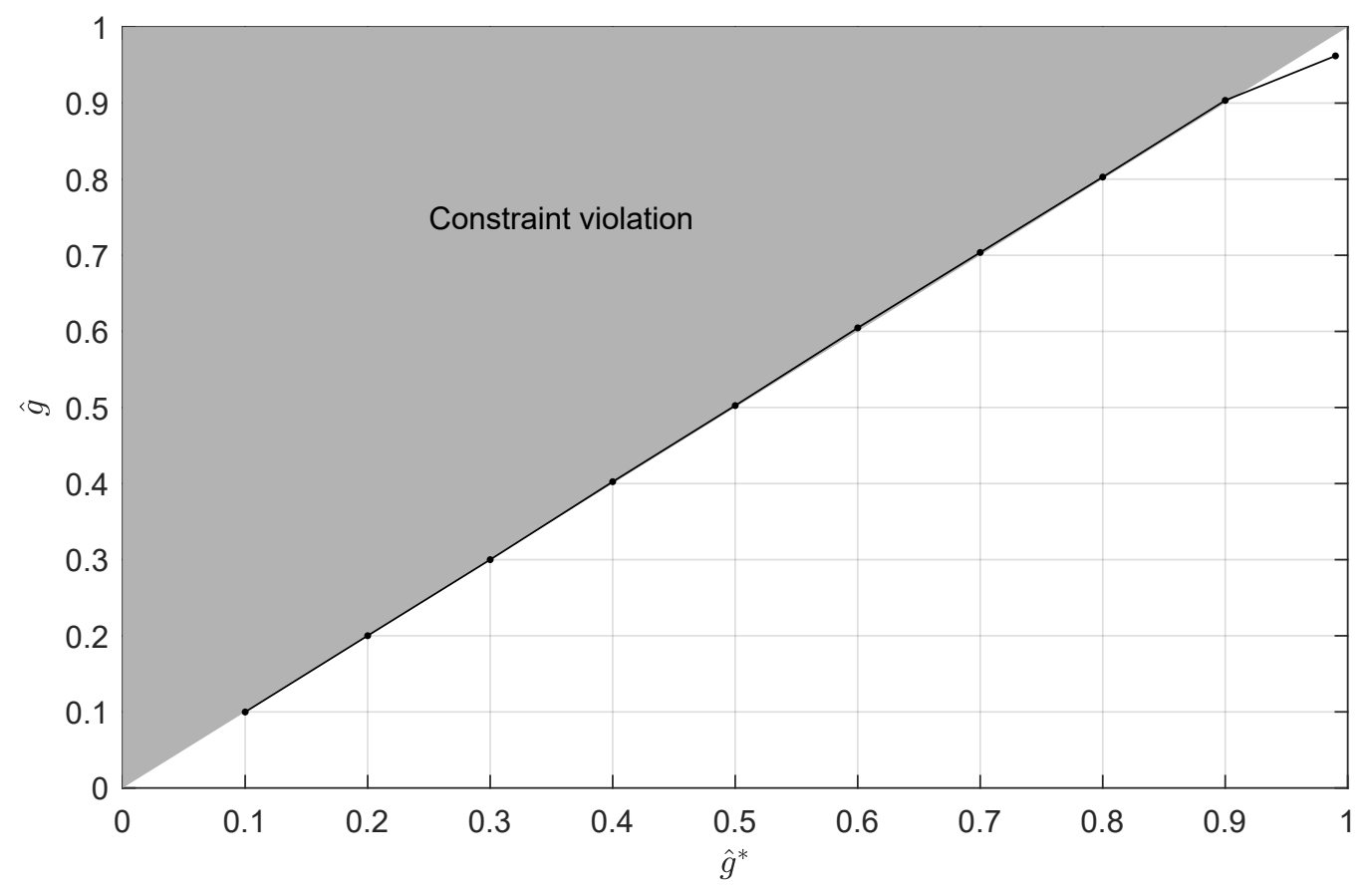

Source: Author

numbers.

For the lowest Reynolds number, $R e=2 \times 10^{5}$, a small strip in the lower surface, close to the trailing edge, is filled with paint: a pattern that is not found for higher Reynolds, which present similar paint patterns. It can be observed by analyzing the cost function that $c_{d}$ is decreasing as the Reynolds number increases, similar and expected behavior of $c_{d}$ when Reynolds number increases in a non-SHS airfoil.

For the internal flow case, the surface constraint is set to $\hat{g}^{*}=0.99$ : as discussed in the last section, it is intended to analyze if the Reynolds number affects the surface covered by SHS when the surface constraint limit is very high. Fig. 70 shows the obtained topology for four different Reynolds numbers and the surface covered by SHS as a function of Reynolds number.

The results show that the Reynolds number, indeed, affects the percentage covered by SHS, and, for the lowest tested Reynolds number, the bottom surface is not fully covered. Figure 71 shows the velocity field divided by the advance velocity $v_{a}$ for each studied Reynolds conditions. For the lowest Reynolds, it can be noted that stagnated velocity and recirculation regions follow the absence of paint distribution: in the bottom part, slightly before and after the corner, the lowest Reynolds case does not present paint, and it is possible to observe that the regions without paint are the regions with lowest velocity/stagnation points. For all cases, in the second corner of the top part, it is observed 
Figure 68 - Wall $y^{+}$distribution along chord for NACA0015 from each resulted topology optimization varying $R e: \alpha=8^{\circ}, \hat{g}^{*}=0.5$, and $\bar{b}=1 \times 10^{-4} \mathrm{~m}$

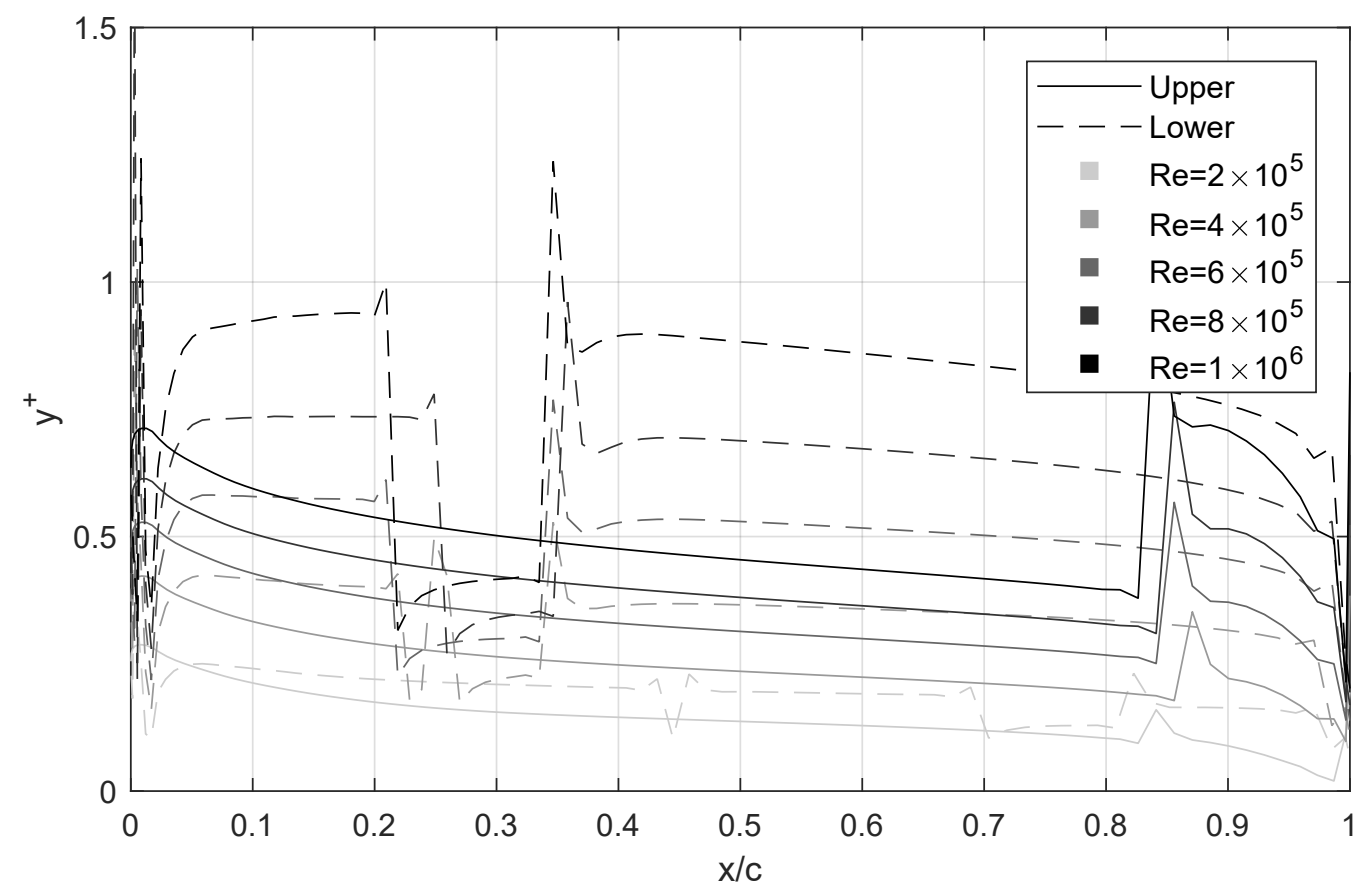

Source: Author

Figure 69 - Comparison of $c d$ (left) and obtained topology (right) for NACA0015 varying $R e: \hat{g}^{*}=0.5, \alpha=8^{\circ}$, and $\bar{b}=1 \times 10^{-4} \mathrm{~m}$
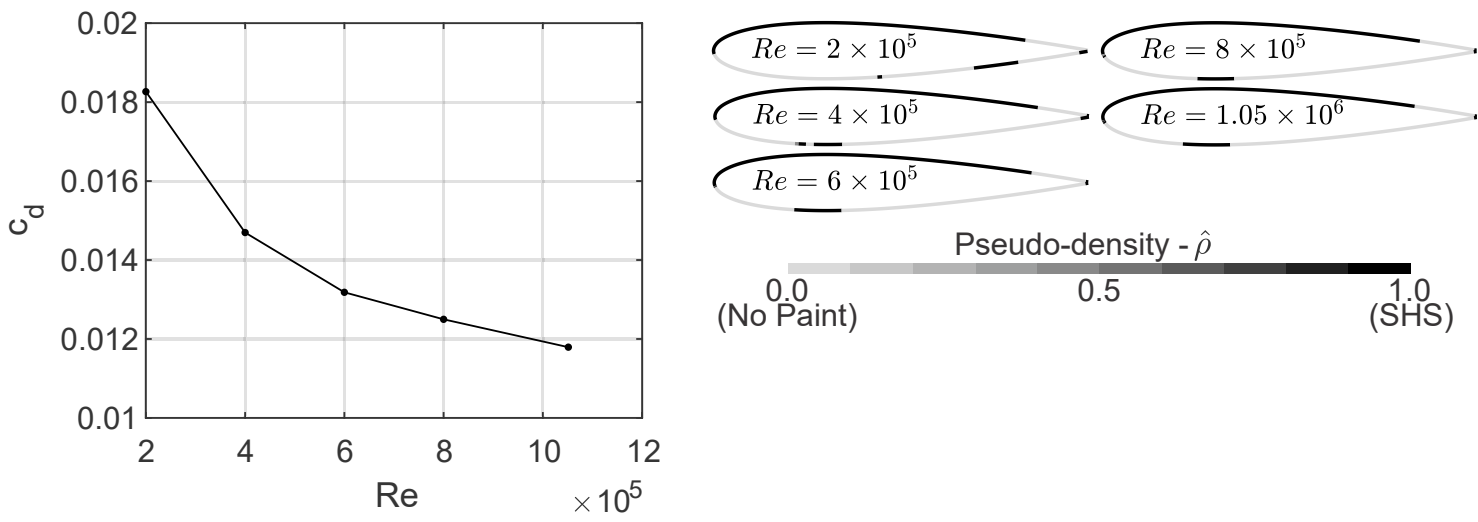

Source: Author

the recirculation zones, followed by the lack of painting.

Since the $R e=1 \times 10^{4}$ case has the lowest turbulence flow, it is less able to sustain an adverse pressure gradient than higher Reynolds cases, once highly turbulent flow supply transport of kinetic energy to the flow close to the wall. As the adverse pressure gradient is related to a decrease of kinetic energy, it leads to a deceleration of the fluid, which may reverse the flow, leading to recirculation zones. In those cases, having an SHS in reverse flow regions is depreciatory for the cost function point of view, since it induces the fluid in the opposite direction of the mean flow. Hence, low Reynolds cases tend to have less SHS, 
Figure 70 - Results for fraction of surface covered by SHS as a function of Reynolds (left) and the comparison of obtained topology (right) for stretched-S pipe varying $R e: \hat{g}^{*}=0.99$, and $\bar{b}=1 \times 10^{-4} \mathrm{~m}$
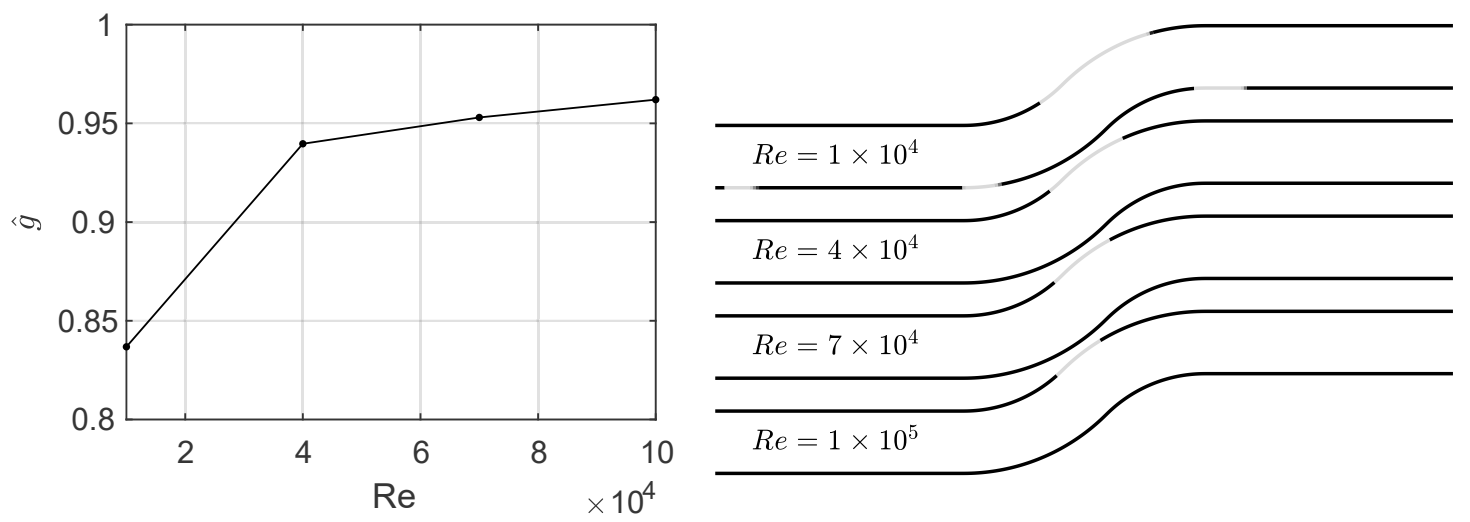

Source: Author

as they have more stagnated and reverse flow regions.

Figure 71 - Stretched-S pipe: velocity field normalized by maximum inlet velocity comparative in region close to corner and comparative of distruted material derived from topology optimization results varying $R e: \hat{g}^{*}=0.99$, and $\bar{b}=1 \times 10^{-4} \mathrm{~m}$

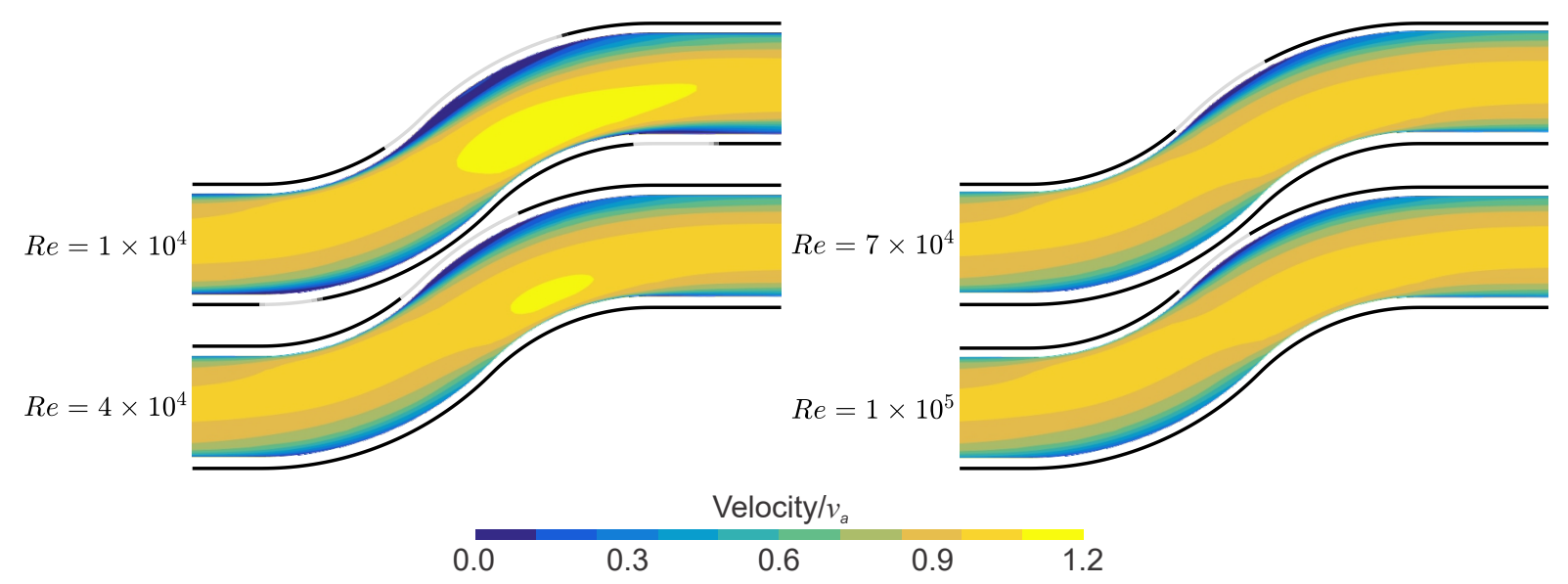

Source: Author

It can be concluded, from the presented cases, that if the Reynolds number is high, the topology is similar. The lowest the Reynolds number, the higher is its influence in the topology. Also, based on the results of the internal flow case, recirculation zones can affect the efficiency of SHS. Therefore, due to the dependency of the Reynolds number into recirculation zones, this effect should be taken into account.

\subsubsection{Influence of angle of attack}

This subsection aims to check the effect of the angle of attack on the SH distribution. Since the study is on the angle of attack, only the external flow is considered. The analyzed 
angles of attacks are from $0^{\circ}$ to $18^{\circ}$, a post-stall region. Figure 72 shows the lift $\left(c_{l}\right)$ and drag $\left(c_{d}\right)$ coefficients (lift coefficient is defined as $c_{l}=2 L / \rho v_{a}^{2} c$, in which $L$ is the lift force per unit of span) for eleven angles of attack, maintaining the other parameters equal, and the obtained topology.

Figure 72 - Comparison of $c_{l}$ (left) and $c_{d}$ (right) for NACA0015 varying $\alpha$ for no-SHS $(\hat{g}=0)$, resulted from topology optimization $\left(\hat{g}^{*}=0.5\right)$ and fully $\operatorname{SHS}(\hat{g}=1.0)$ : $R e=1.05 \times 10^{6}$ and $\bar{b}=1 \times 10^{-4} \mathrm{~m}$

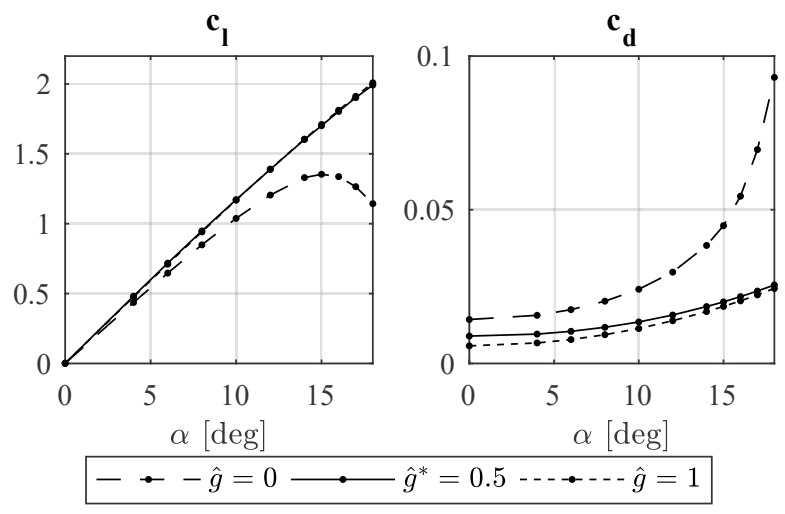

Source: Author

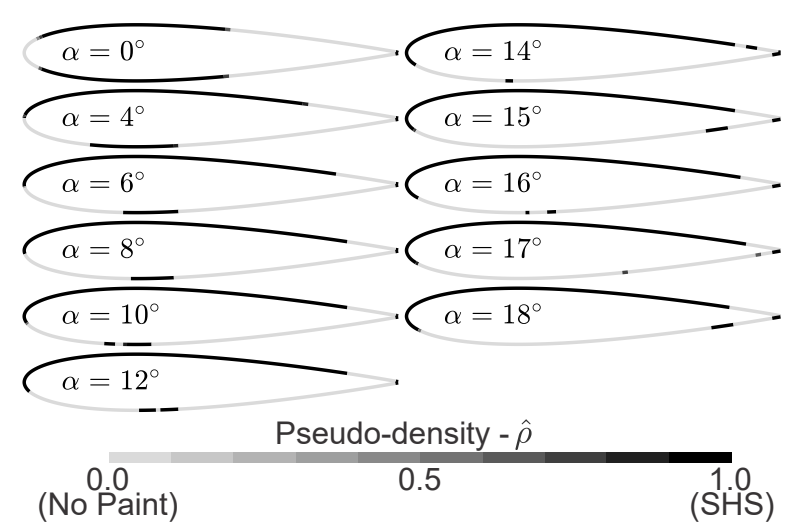

As expected, for $\alpha=0^{\circ}$ the topology is symmetric. For angles of attack before the stall condition in no-SHS case, before $\alpha=12^{\circ}$, the painting distribution also does not change significantly, even when changing pressure and shear stress distributions. The results show that, despite changing the flow condition in the foil, the optimized topology remains roughly similar for the no-stall conditions. With the result obtained, the most expected hypothesis is that there are no major topology differences for small values of angle of attack, before the stall.

However, for angle of attack in which it can be observed that stall condition is closer (by observing the lift coefficient $c_{l}$ of no-SHS case from Fig 72), it is observed that the small stripe close to the leading edge disappears and the topology prioritize the upper surface, in the direction of the trailing edge. To understand this phenomenon, Fig. 73(left) shows the velocity field and streamlines for the angle of attack slight before the stall angle and Fig. 73 (right), the highest angle of attack optimized. Both figures show the velocity field for three conditions: no-SHS, resulted from optimization with surface constraint limit of $\hat{g}^{*}=0.5$ and fully SHS.

Figure 73 (left) shows the effect of SHS in the upper surface: for $\hat{g}=0.0$, a small recirculation zone close to trailing edge is noted, whereas for $\hat{g}^{*}=0.5$ and $\hat{g}=1.0$ it is not observable. The typical adverse pressure gradient in the upper surface decelerates the flow in which can lead to zero or reversed velocities, causing the detachment of fluid flow. However, on SHS the dissipated energy from shear stress is reduced, leading to a higher net momentum close to the wall, decreasing the deceleration rate of fluid flow. It can be 
Figure 73 - NACA0015 velocity field normalized by far-field advance velocity $v_{a}$ for $\alpha=12^{\circ}$ (left) and $\alpha=18^{\circ}$ (right). Streamlines comparison for no-SHS ( $\left.\hat{g}=0\right)$, resulted from topology optimization $\left(\hat{g}^{*}=0.5\right)$ and fully $\operatorname{SHS}(\hat{g}=1.0)$ : $R e=1.05 \times 10^{6}$ and $\bar{b}=1 \times 10^{-4} \mathrm{~m}$
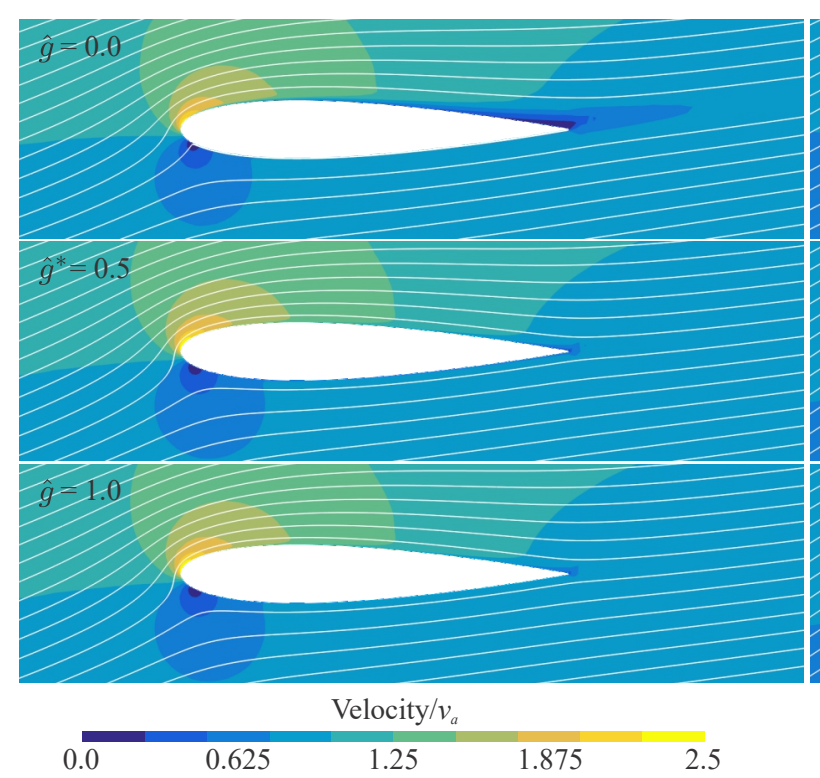

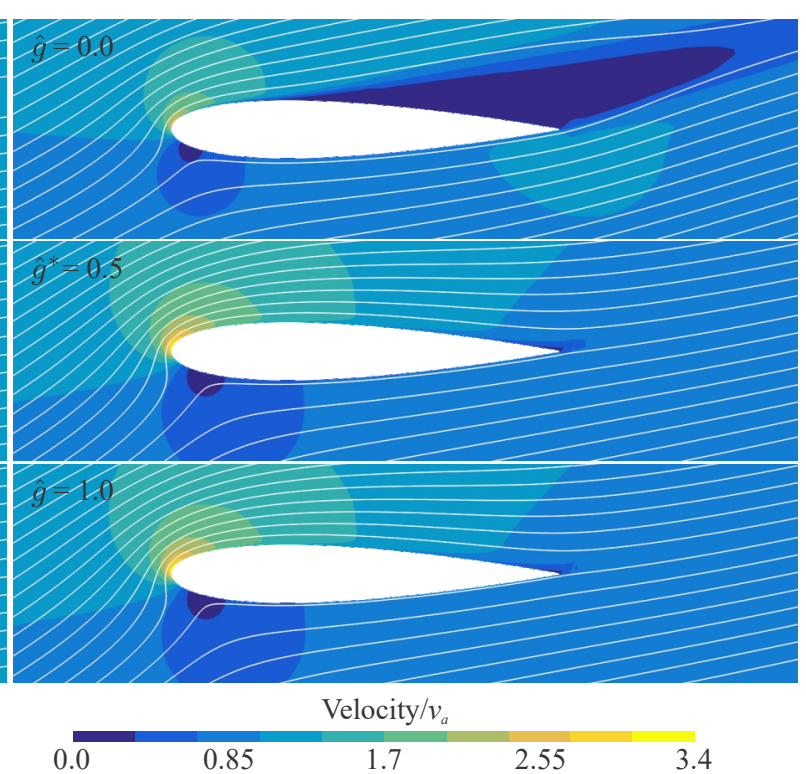

0.0
1.7

3.4

Source: Author

observed for the case of $\alpha=12^{\circ}$ that this decrease of deceleration avoids the recirculation zones. Figure 73 (right) shows a condition in which is clearly a stalled condition for $\hat{g}=0.0$, which leads to elevated values of $c_{d}$. By having SHS in the upper surface, the fluid flow remains attached and the stall condition is postponed, avoiding high quantities of dissipated energy due to vorticity.

The flow detachment leads to high vorticity zones and high values of the drag coefficient. The optimization process focuses on the postpone of the detachment by avoiding the dissipated energy from shear stress and, in this way, increasing the net momentum close to the boundary layer. In conditions in which the no-SHS case is far from the stall angle, the optimization focuses on drag reduction by applying SHS on the upper surface and a small stripe in the leading edge. As the stall angle becomes closer and the adverse pressure gradient gets high, the optimization process focuses on keeping kinetic energy as higher as possible by decreasing the dissipated energy from shear stress. Therefore, SHS starts to be replaced from the lower surface to the upper surface, to keep the attachment of fluid flow as long as possible.

It can be interpreted as, for low angles of attack, the main drag contribution is the shear stress. In this sense, the optimization focus on reducing the shear stress term of drag, whereas the higher angles of attack, as vorticity increases the drag considerably, the main objective is to postpone stall. Also in higher angles of attack, the pressure-term 
contribution becomes higher, so the main function of SHS is not to reduce the skin-friction drag, but postpone the stall. It can be proven by observing Fig. 72 that the $c_{d}$ between half of the foil covered by SHS and fully SHS is not so distant for a higher angle of attack, once $\hat{g}=0.5$ is enough to postpone the stall, as observed in Fig. 73 (right). Thus, a foil with SHS in the upper surface is important, once it can postpone stall, but for high angles of attack and $\hat{g}>0.5$ (i.e., having SHS in the lower surface) could be not so effective, once the drag is predominated by pressure-term and, despite the stall postpone function, decreasing the skin-friction drag on the lower surface has small impact in the overall drag composition.

It is important to observe that the stall is a three-dimensional and unsteady phenomenon. In this way, the results show an approximation of the expected result in stall condition. Besides, a study of the mesh refinement would be interesting to check the correct modeling of eddies present in post-stall flows. Simulating post-stall conditions with accuracy is still a difficulty nowadays. Slotnick et al. (2014) cites flow separation as CFD technology gaps and impediments, as all types of separated flows are still challenging to predict, and it will remain a pacing item by 2030. However, since the RANS model is adopted, and the main objective is in the average, macroscopic analysis of the flow, aiming to observe the flow separation, the stall point, and the lift and drag of the foil, it is assumed that the mesh and the adopted models are able to estimate the hydrodynamic at the post-stall condition well. The obtained results in this work show the potential of the optimized SHS distribution. The results obtained here, especially in post-stall regions, encourage further investigations and experimental validations. 



\section{Topology Optimization Results: Propeller}

\section{case}

This chapter applies TOM to increase the hydrodynamic efficiency of the propeller by defining SHS regions on the blade, in which the maximum amount of paint is limited. The chosen condition is $J=0.7$ and $n=1600 \mathrm{RPM}$, resulting in $R e_{p}=5.70 \times 10^{6}$, once it represents a condition close to the maximum efficiency. The sensitivity formulation is deduced and presented in Sec. 4.6. Each optimization iteration takes considerable time since it is a three-dimensional simulation, and needs to run the primal and adjoint problems. Some tests and discussions of applying the TOM in SH problems are conducted in Sec. 8.

Firstly, the topology optimization formulation is presented and the results are discussed. As a part of the procedure, the results are post-processed to get paint distribution. Since the adopted mesh is designed to be used inside the optimization framework, the filtered-topology solution is transposed to a finer mesh, that was compared with experimental data in Sec. 6. Finally, the resulted topology optimizations are compared with no-SHS condition and also in a condition in which it is possible to cover the blades fully.

\subsection{Topology of SHS distribution}

The optimization problem is to maximize the hydrodynamic efficiency $\eta$. Since the optimizer works with minimization problems, the cost function is multiplied by -1 , as shown below:

$$
\begin{array}{|ll}
\operatorname{Min}_{\hat{\rho}} & C=-k_{s} \eta=-k_{s} \frac{T v_{a}}{2 \pi n Q} \\
\text { s.t. } & \\
& \hat{g}(\hat{\rho})=\frac{\int_{\Theta} \hat{\rho} d s}{\int_{\Theta} d s} \leq \hat{g}^{*} \\
& 0 \leq \hat{\rho} \leq 1
\end{array}
$$

In the presented formulation, it is adopted a value of $\hat{g}^{*}=[0.3,0.5,0.7]$. Material properties are $\bar{b}=1 \times 10^{-4} \mathrm{~m}$ and penalization $q=4.0$. The initial condition is $\hat{\rho}=0.1 \mathrm{in}$ whole surface. Next figures show the optimization process as a function of iteration for each case of $\hat{g}^{*}$ and topologies obtained in some iterations.

It can be observed that in the last iterations there is a slow growing of SHS (i.e. 
Figure 74 - Propeller efficiency $\eta$ and surface-average value of pseudo-density $\hat{g}$ along optimization iterations for $\hat{g}^{*}=0.3$

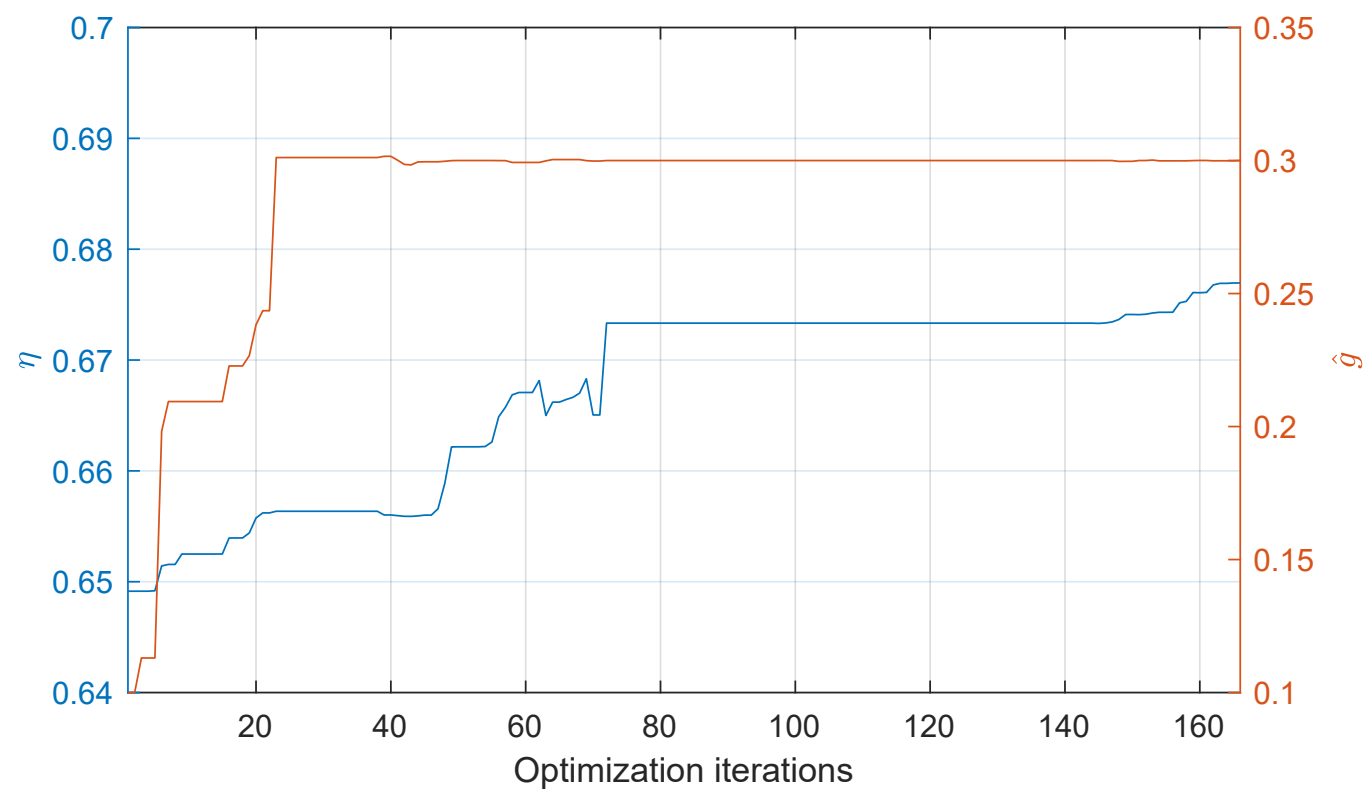

Source: Author

Figure 75 - Topology evolution along optimization iteration for $\hat{g}^{*}=0.3$

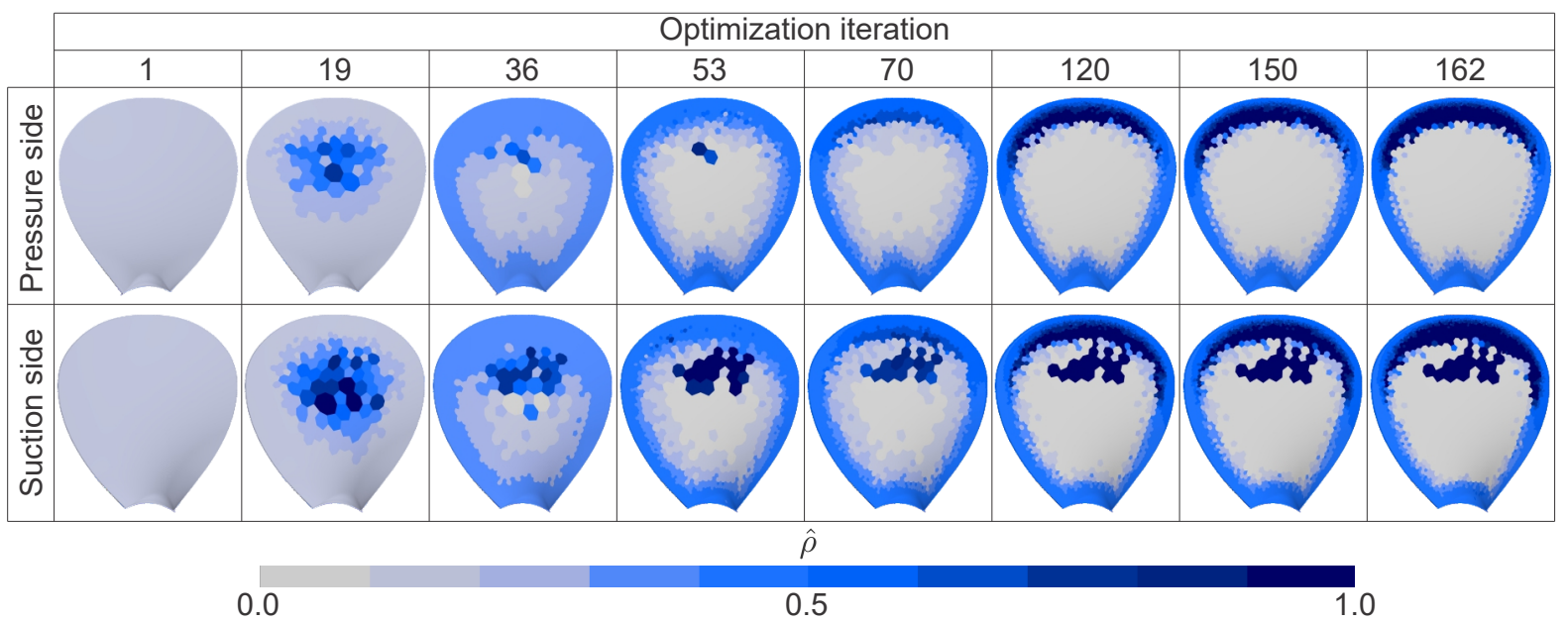

Source: Author

$\hat{\rho}=1.0$ ) into the edges. It can be seen specially in $\hat{g}^{*}=0.5$ condition, Fig. 77 , between optimization iteration 108 and 150. Since there are more elements on the edge, this movement takes more iteration compared to the center blade region. 
Figure 76 - Propeller efficiency $\eta$ and surface-average value of pseudo-density $\hat{g}$ along optimization iterations for $\hat{g}^{*}=0.5$

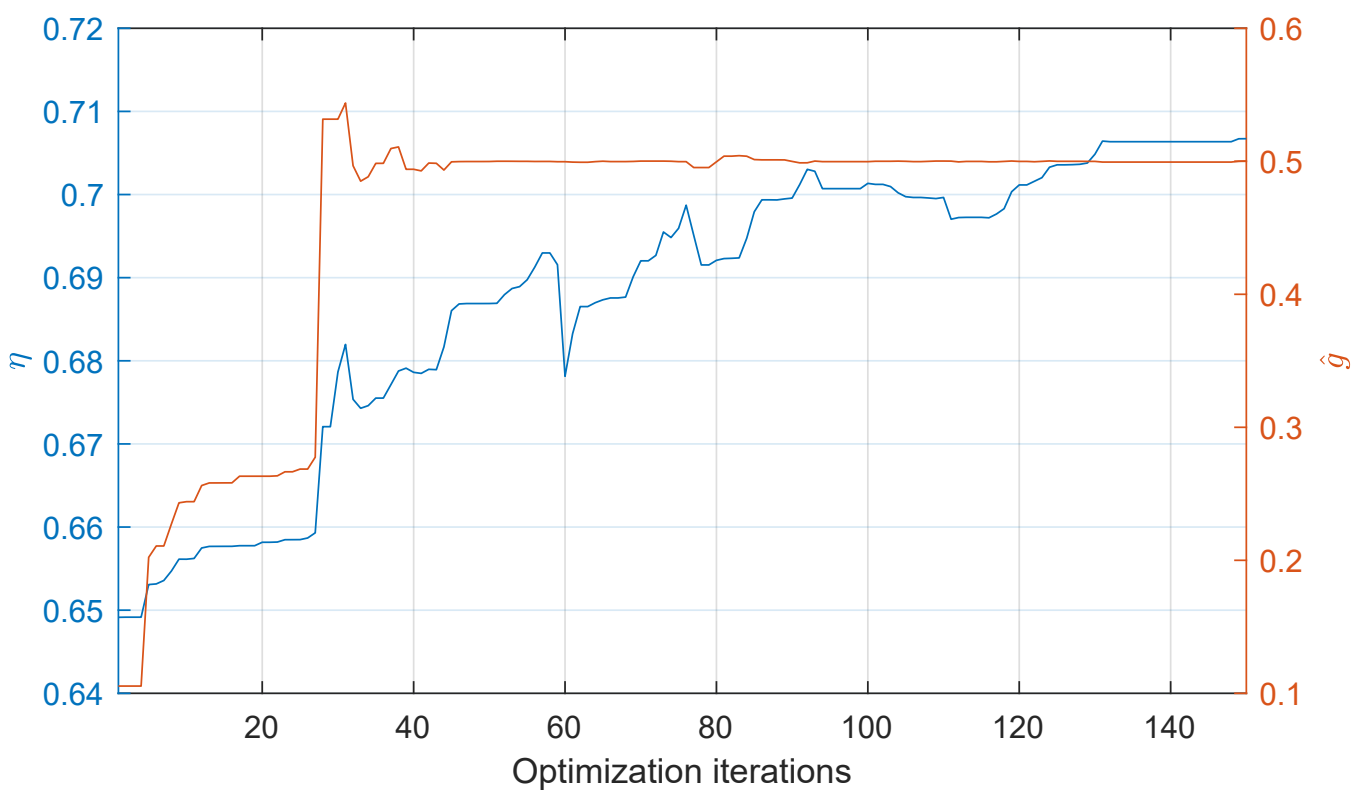

Source: Author

Figure 77 - Topology evolution along optimization iteration for $\hat{g}^{*}=0.5$

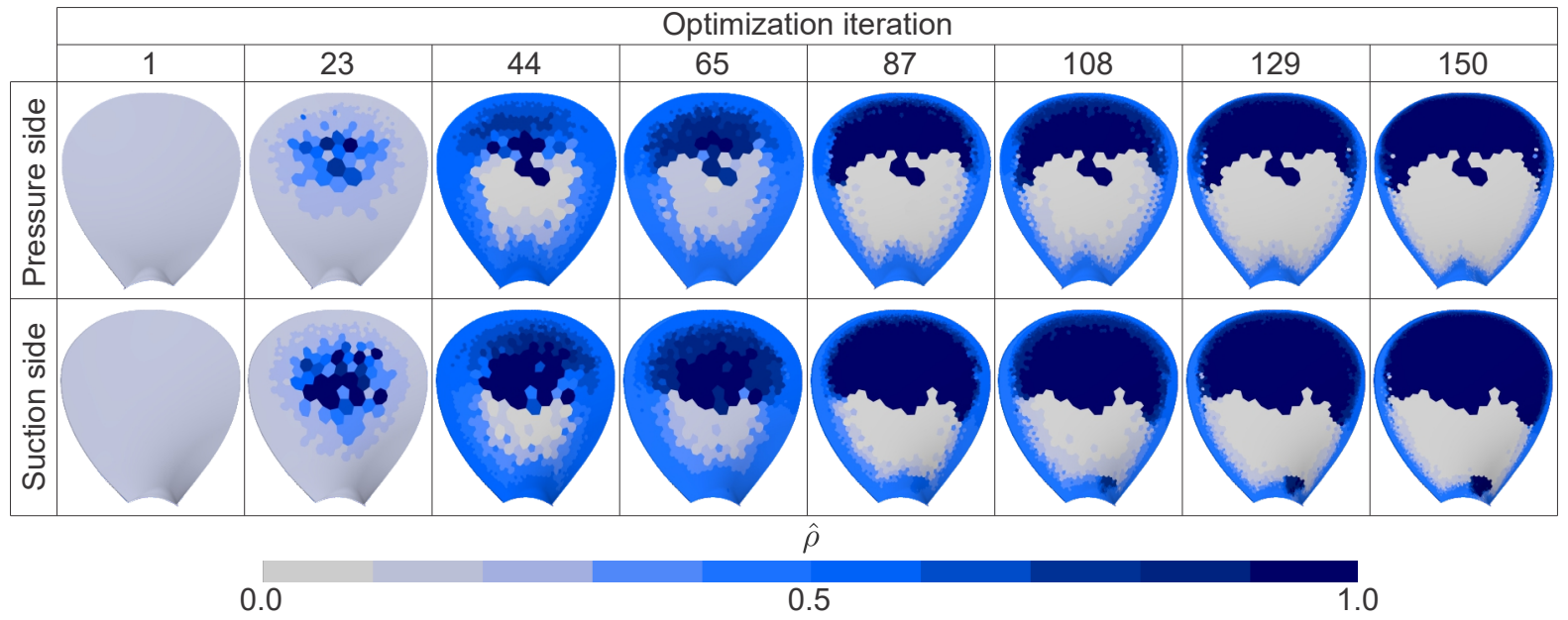

Source: Author 
Figure 78 - Propeller efficiency $\eta$ and surface-average value of pseudo-density $\hat{g}$ along optimization iterations for $\hat{g}^{*}=0.7$

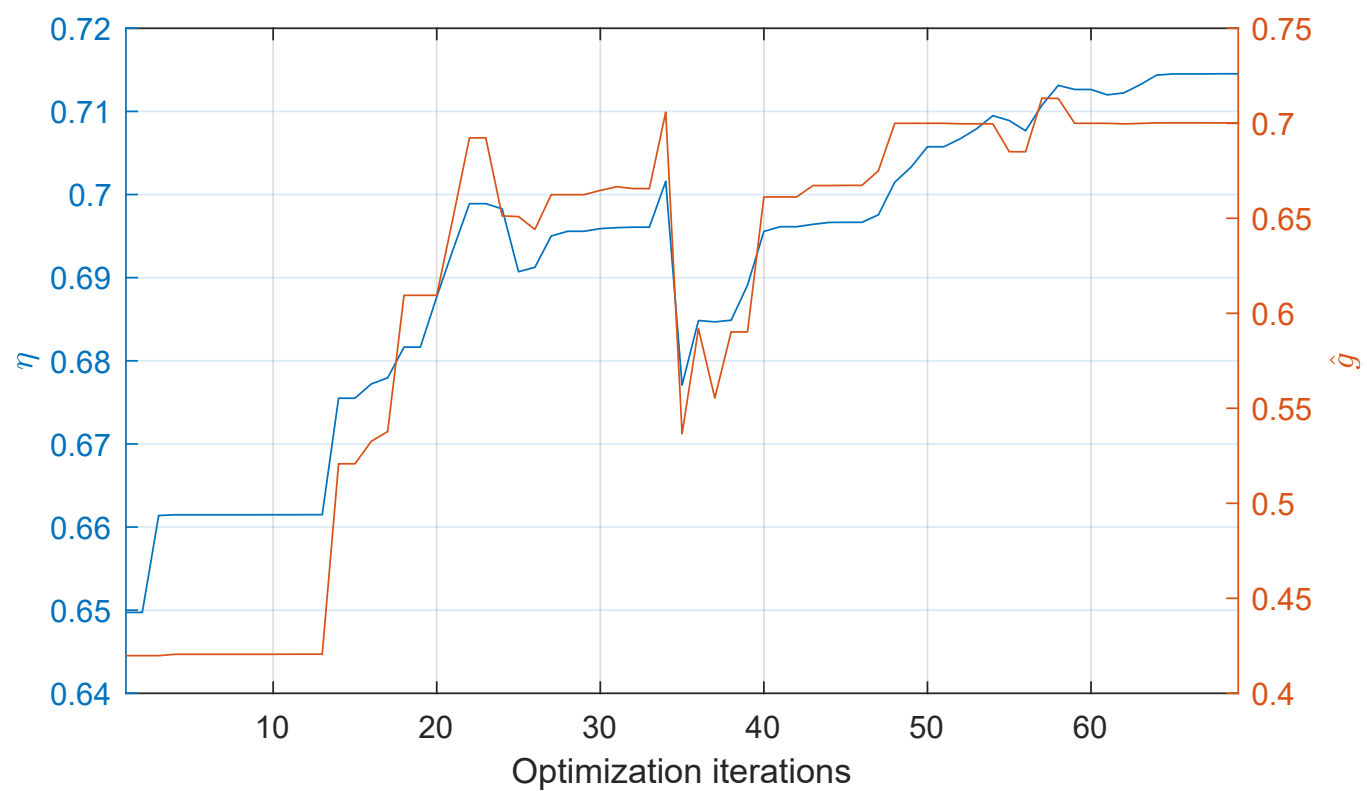

Source: Author

Figure 79 - Topology evolution along optimization iteration for $\hat{g}^{*}=0.7$

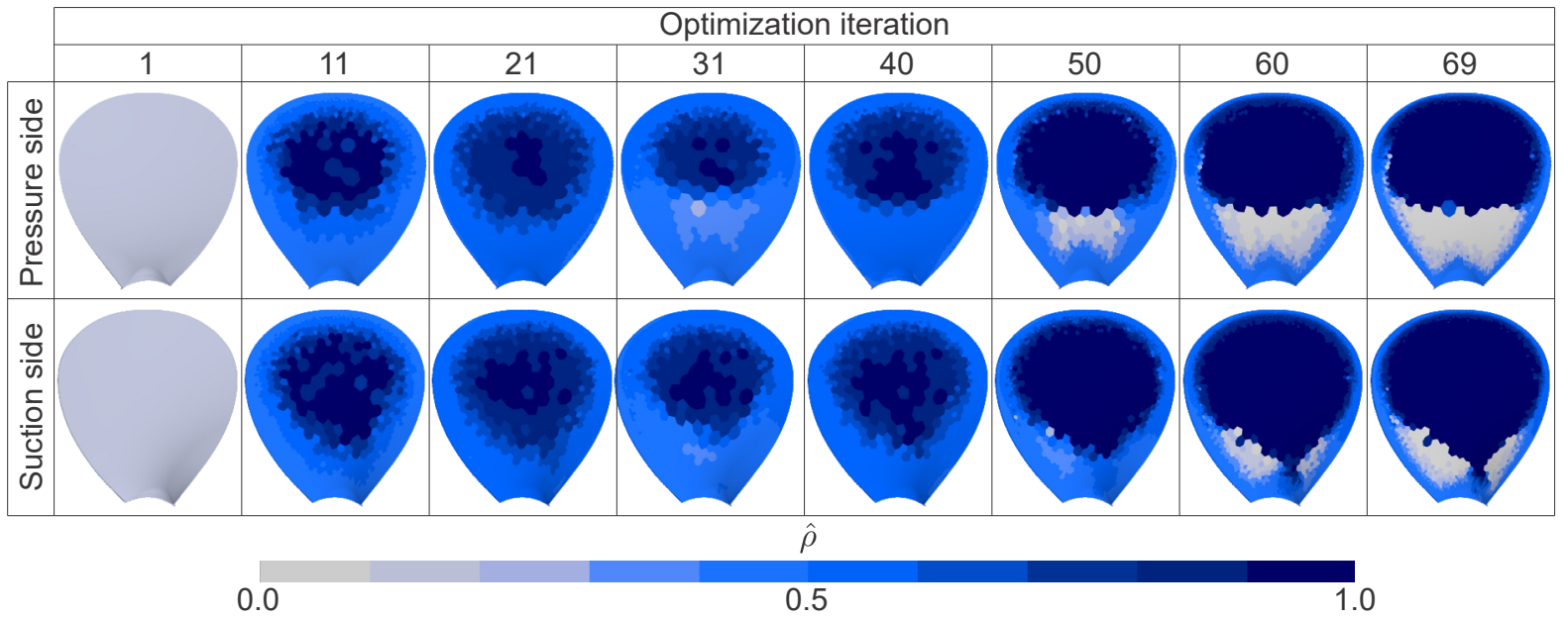

Source: Author 


\subsection{Post-processing}

The results must be post-processed in order to indicate which regions should be covered with SH painting. This post-processing is part of the design procedure discussed in Sec. 3.2 and it is done by applying a filter-like process: all values of pseudo-density above a threshold are equal to one and all below are equal to zero. The threshold is chosen such that it still satisfies the surface constraint $\hat{g}$.

Figure 80, Fig. 81 and Fig. 82 show topology optimization solutions and theirs postprocessing results after treatment procedures for cases with $\hat{g}^{*}=[0.3,0.5,0.7]$, respectively. The obtained value of threshold is around 0.5 for all cases, as expected, since it corresponds of the mean value of range of $\hat{\rho}$.

Figure 80 - Obtained topology from optimization process and after post-process for $\hat{g}^{*}=$ 0.3

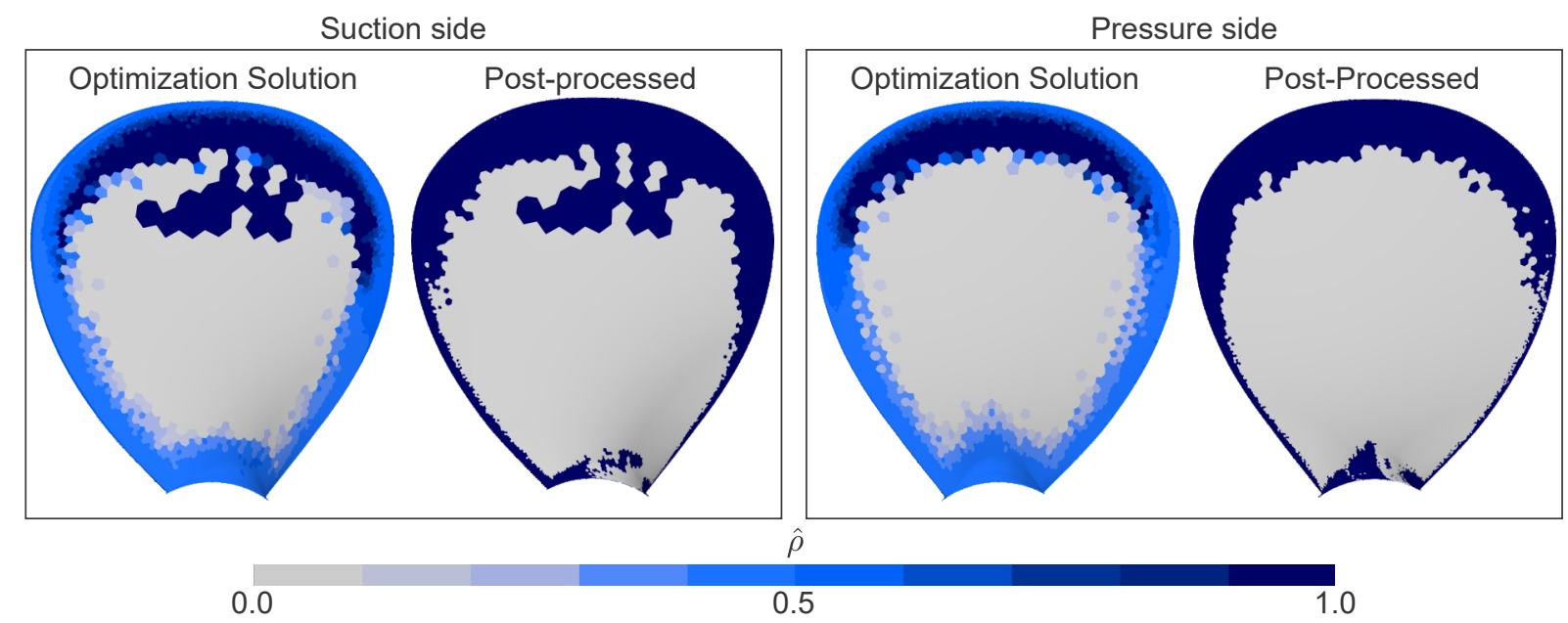

Source: Author 
Figure 81 - Obtained topology from optimization process and after post-process for $\hat{g}^{*}=$ 0.5

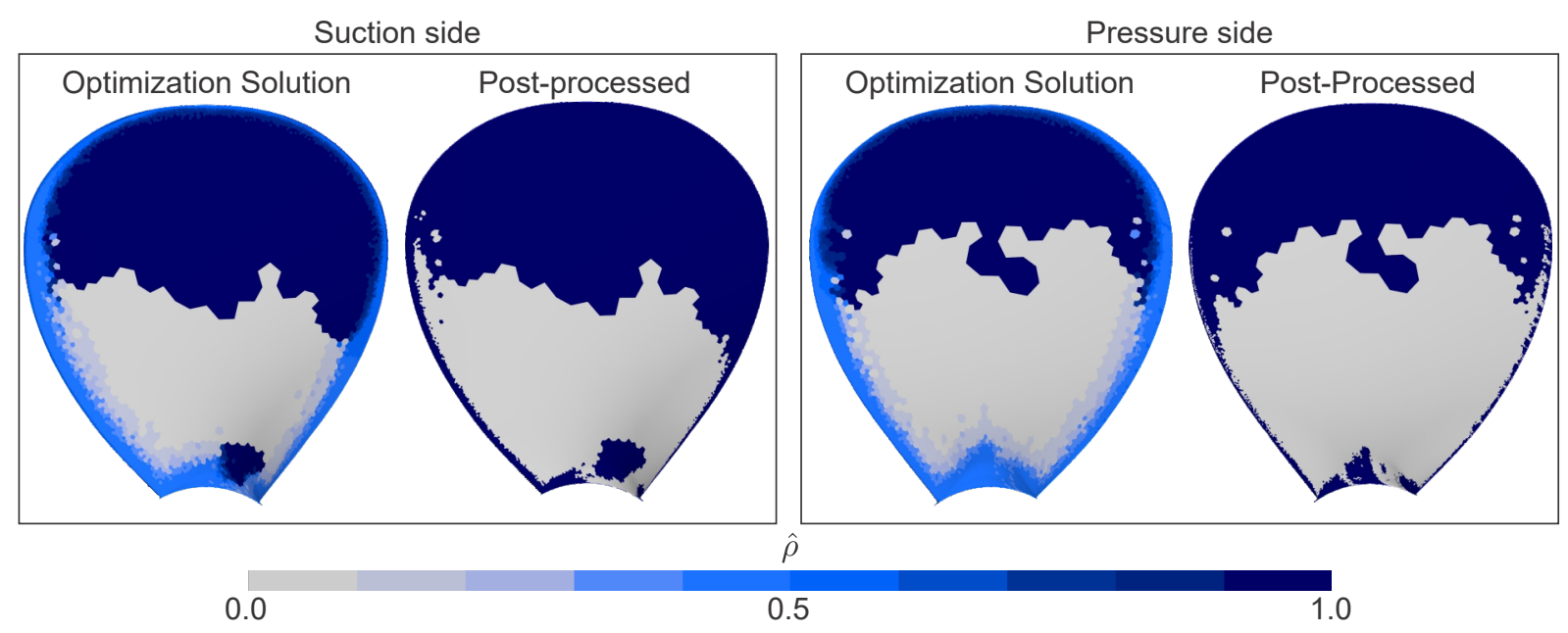

Source: Author

Figure 82 - Obtained topology from optimization process and after post-process for $\hat{g}^{*}=$ 0.7

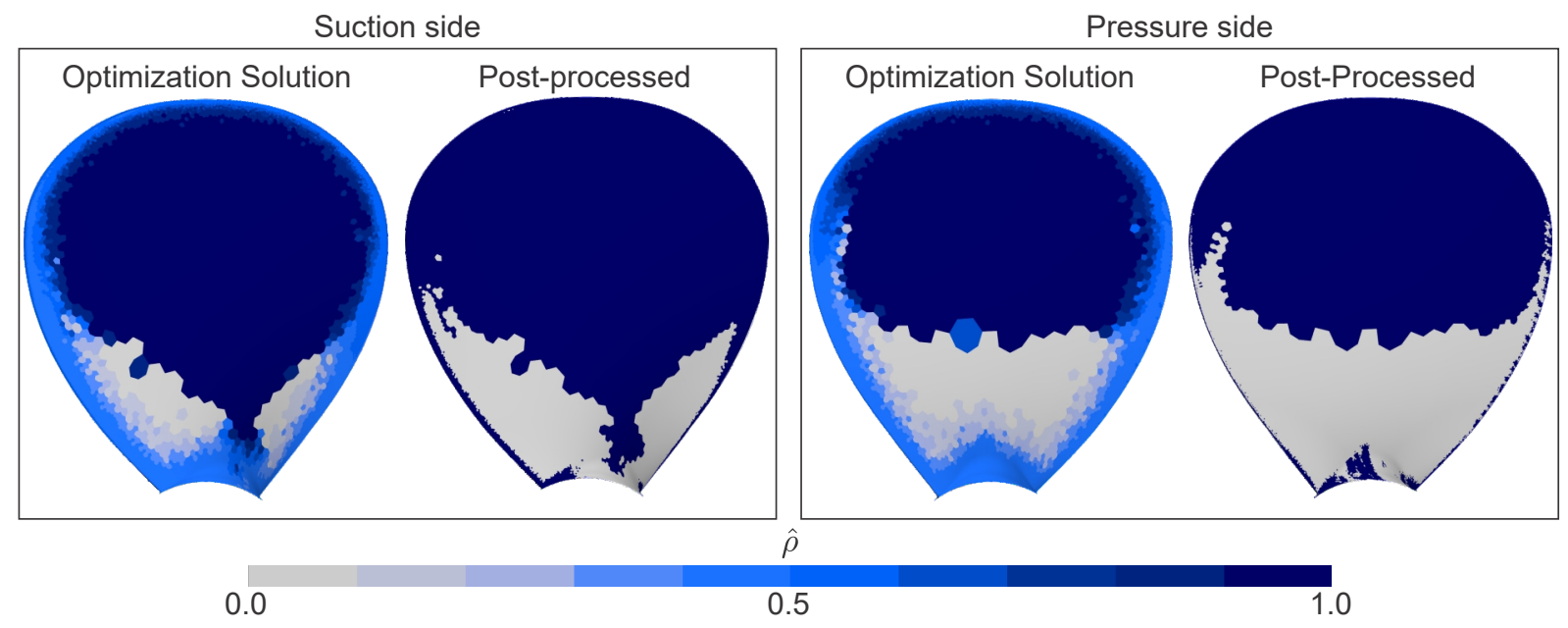

Source: Author

\subsection{Finer mesh comparison}

Several designs of SHS distributions are tested during the optimization process, requiring a faster evaluation of cost function. In this work, since it is employed CFD tools, it is used a coarse mesh to enhance the consumed time per cost function evaluation. As noted in Sec. 7, in a full-SHS scenario, this polyhedral mesh produces results with the non-negligible difference from the $\mathrm{hr} 2$, a more refined hexahedron mesh. 
The hr2 mesh, which has its results validated with experiments, would be appropriate to be used in the optimization framework, but the computational cost would make it infeasible. Instead, a coarser mesh is used, and the result of the painting distribution is mapped into the finer mesh, hr2. This mapping is done using nearest-neighbor (closes point) interpolation from cell-centered data of coarse mesh topology. Getting hydrodynamic performance values using the same hr2 mesh makes it possible to perform a fair comparison of the gain of hydrodynamic efficiency between a propeller without any SHS, with topology optimization design and fully covered by SHS. Figure 83 shows the filtered topology result using the coarse mesh and the result of its mapping to the most refined mesh.

Figure 83 - Mapping results from polyhedral (used in optimization framework) to hexahedron hr2 (finer mesh, to be used to compare with previous results)
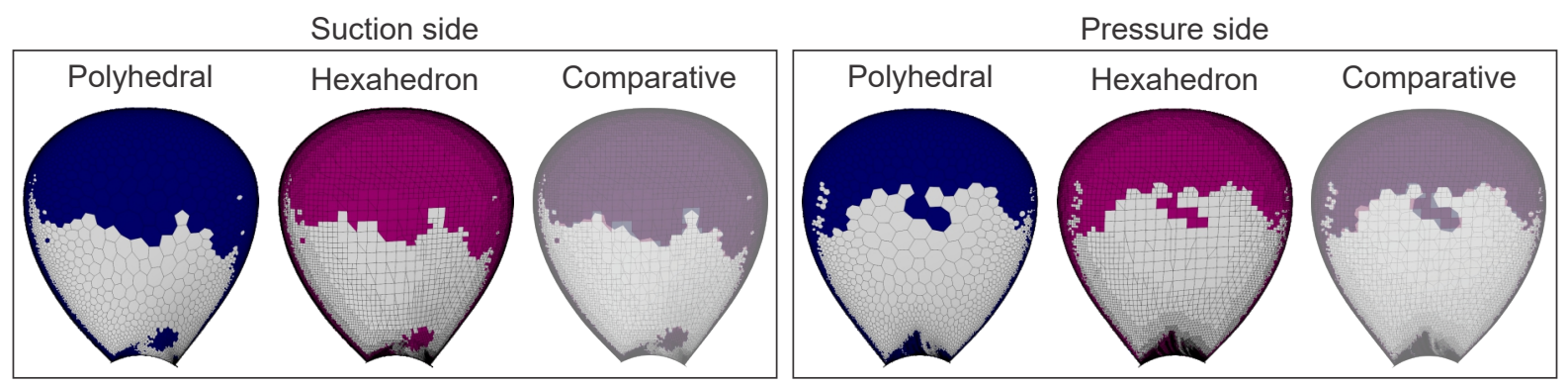

Source: Author

Figure 84 shows a comparative between polyhedral (coarse) and hexahedron (fine) mesh for Coefficient of Pressure $C p$.

Figure 84 - Comparative of $C p$ between coarse and finer mesh
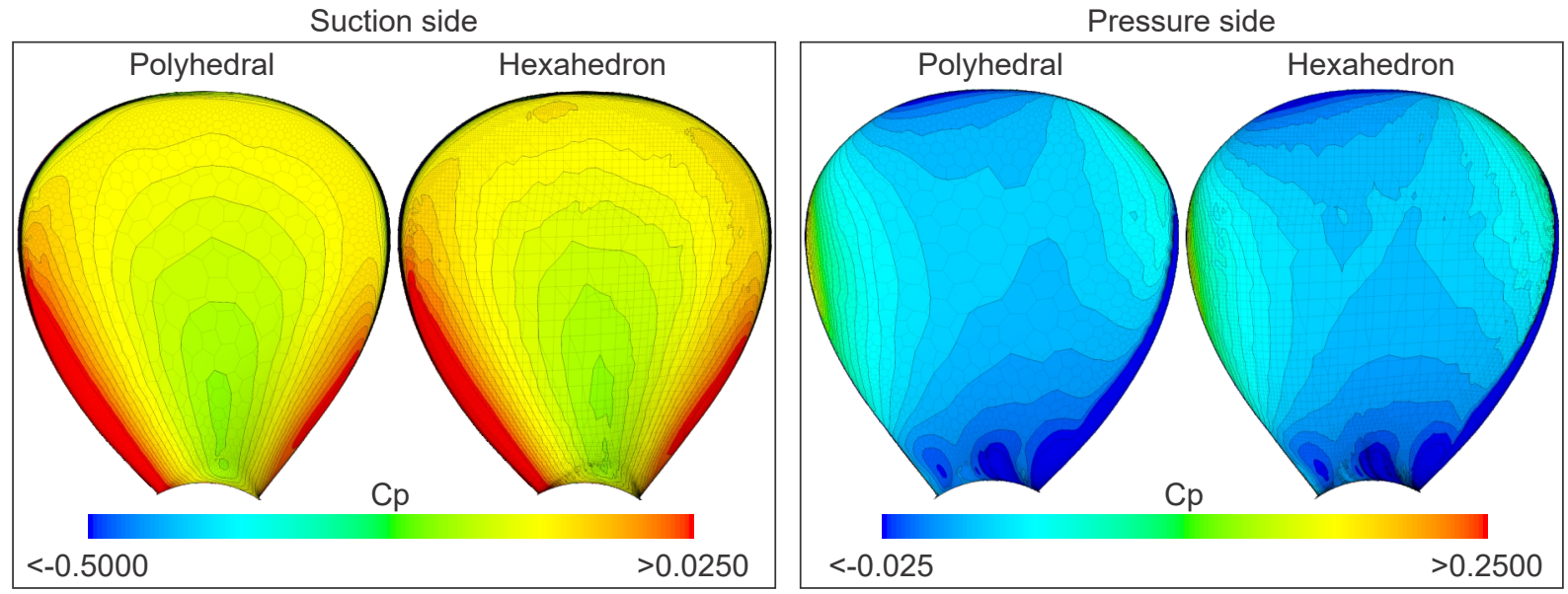
Indeed, by analyzing the distribution of $C p$ on the blade, it does not lead to a considerable difference. Table 6 shows the value obtained by each mesh and the percentage of difference.

Table 6 - Comparative of propeller coefficients between Optimization mesh (polyhedral) and hr2 (hexahedral), $J=0.7$

\begin{tabular}{lccc}
\hline Coefficient & Polyhedral/Coarse & Hexahedron/Fine & Difference [\%] \\
\hline$K T$ & 0.1230 & 0.1178 & $4.20 \%$ \\
$K Q$ & 0.0193 & 0.0181 & $6.26 \%$ \\
$K Q s$ & 0.00100 & 0.00108 & $-7.83 \%$ \\
$\eta$ & 0.7100 & 0.7253 & $-2.16 \%$ \\
\hline
\end{tabular}

in which $K Q s$ is the torque coefficient, but considering only the shear contribution.

\subsection{Comparative with no-SHS and full-SHS}

From the mapped topology solution, the result can be compared with no-SHS and full-SHS condition using the same mesh. Figure 85 shows the efficiency gain, the main composition for the cost function expression used in the optimization framework.

Figure 85 - Efficiency in function of surface constrain adopted in optimization process and comparison with no-SHS $(\hat{g}=0)$ and full-SHS $(\hat{g}=1)$

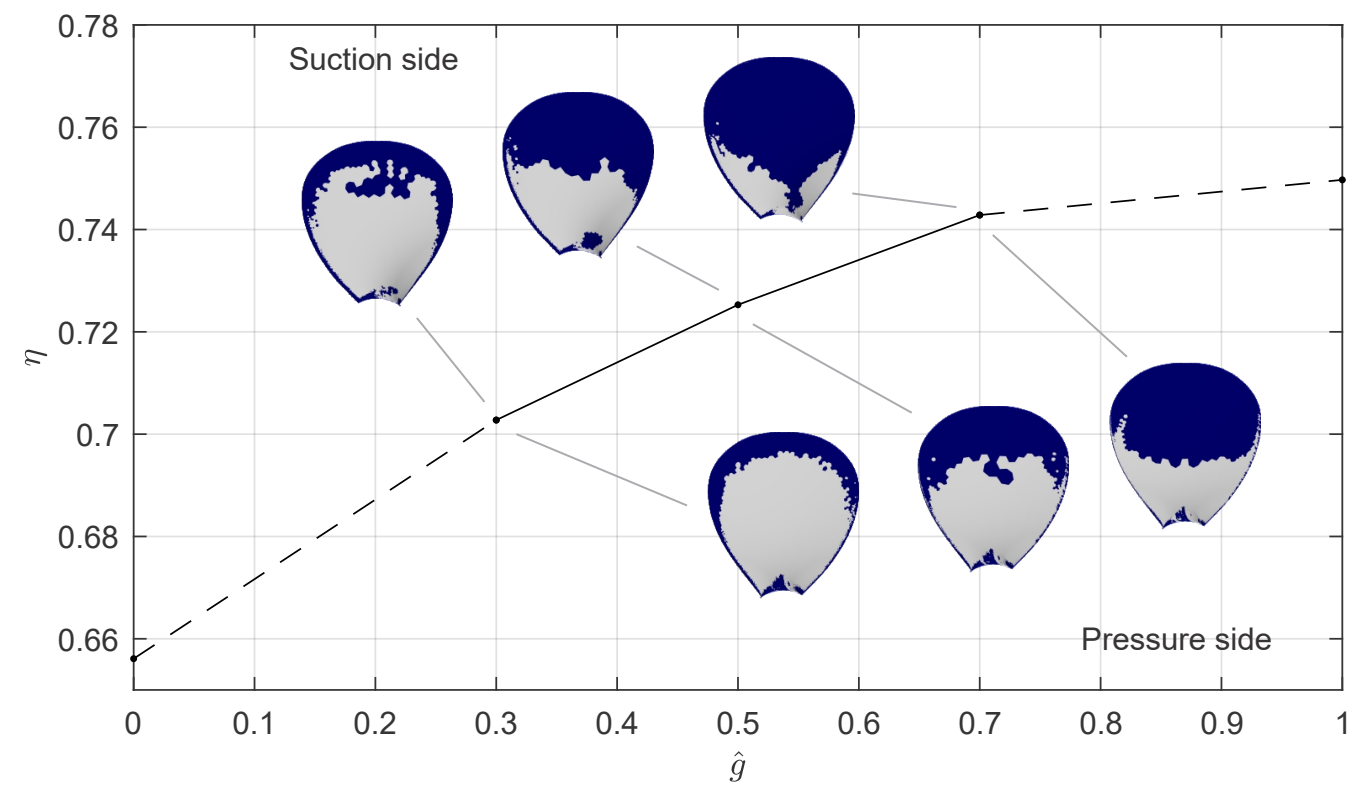




\subsection{Discussions}

The general topologies obtained from the optimization process are regions covered at the propeller tip and extending toward the center. It is also possible to notice the presence of SHS, in smaller quantity, in the region near the axis. This result also endorses the choice of topology optimization, since such generality of design would be very complicated or even impossible in other types of optimization methods.

Also, it can be observed that, in general, the starting radius of painting is different between suction and pressure surfaces. As expected from 2D topology optimization results, the suction surface has a larger SHS area than the pressure surface.

In this problem, the cost function is only to increase hydrodynamic efficiency. If it would also be considered other factors such as the cost of painting, a maximum trade-off point could appear without a painting limitation.

Methods for creating an SHS can vary from a sprayable-painting to a corrosioncontrolled process on a propeller surface to create micro-surface elevations and traps small air pockets. In any case, it is reasonable to consider that the cost of creating an SHS could be given by a linear function with the area of SHS. Because of the well-defined function, obtaining its value and its derivative would be easy. Also, to counterbalance the cost function, cost reduction may be included as a function of increasing propeller efficiency, which would take into account the price of engine fuel, for example. This approach is not included in this work since several steps before should have been done. It should include better investigating on the tribology to create an SHS and better understanding of how to formulate an expression to consider the cost of manufacturing an SHS in the model-scale propeller. As stated, the objectives of this work do not extend to investigate the modeling or practical manufacture of SHS, but to use a well-known model and apply it to a topology optimization problem in turbulent flows. Because of this, it is chosen to impose a paint constraint to understand which regions to prioritize. In this way, it is possible to observe the growth of the optimizer prioritization, showing where the most critical regions in the propeller are. By studying three cases by varying the surface-constraint, it is observed that the propeller tip should be prioritized and, in smaller quantities, regions near the axis. 



\section{Conclusions}

This work proposes another way to use Topology Optimization: given a fixed propeller geometry, design a surface distribution of an $\mathrm{SH}$ painting given a cost function and constraints. Unlike most topology optimization applications applied to fluid problems, since the geometry is fixed, it is possible to use turbulence models more easily. Turbulent flow modeling is difficult to be used within Topology Optimization since eddy viscosity models are strongly dependent on wall distance. Since, by not having a well-defined wall, most of classical Fluid Topology Optimization problems lack on this problem by trying to approximate the Turbulence equations or estimate the wall distance.

A novel derivation of sensitivity on the surface based on a discrete adjoint solver is shown. Using an external CFD code (in a black-box concept) that contains a primal and adjoint solver, the developed formulation uses the solution of each volumetric element and convert to get the sensitivity on a surface. It is chosen to use an external CFD code instead of developing code to focus on the derivation of sensitivity and topology optimization. Also, by developing a framework with an external CFD code, we get flexibility for using it with other applications, other different models, or even other CFD codes.

A method for propeller simulations is defined and validated with results from Cavitation Tunnel tests. It is observed that a cylinder domain with radius five times the propeller radius and length 20 times the propeller radius has good results with a feasible computational cost. The region in which refining is important for convergence and good results are defined, concluding that a hexahedron mesh with about 3.7 million elements produces satisfactory results. In the turbulence models, four models were tested, which resulted in close values with each other, choosing $k-\omega$ SST model. Three different cases of cavitation index, with six operating conditions in each, are simulated and is observed that the numerical procedure adopted generated in results close to the measurements in the Cavitation Tunnel, even in conditions with large cavitation area. The method presented is used for the simulation with SHS and the adjoint problem.

It is studied the numerical effect of an SHS in 2D (internal and external flow) and 3D (propeller) cases. Some numerical experiments are performed for two-dimensional internal and external flow cases, by varying the slip length, Reynolds number, surface constraint, and angle of attack. It has been observed that the slip length $b$ does not significantly change the topology of SHS distribution. Thus, not having an exact value of $b$ does not significantly impact the obtained topology from optimization. Since low Reynolds turbulent flows are more likely to present stagnation or reverse flow, apply SHS in these regions can be detrimental to the drag reduction. Indeed, it is observed that the reverse 
flow regions are not covered with SH paint, as shown by the results from the optimization process. For external flow, it is observed that the topology does not change considerably for low angles of attack. When reaching angles close to the stall, the topology optimization results start to focus on postponing stall, by adding SHS on the upper surface. In addition, for a higher angle of attack, the pressure-term contribution to drag increases, making the skin-friction reduction from SHS less notable. In this way, it is observed that for low angles of attacks, optimization tries to reduce drag by decreasing the skin-friction term. For higher angles of attack, optimization aims to avoid the flow separation by trying to maintain a high momentum close to the boundary layer on the upper surface.

In the propeller topology optimization case, three surface constraint cases are performed. A tendency can be observed to prioritize the distribution of SHS on the propeller tip and, in a smaller quantity, on regions near to the axis. Also, confirming the result obtained in the foil case, the suction surface is prioritized over the pressure surface.

Some side-consequences of developing this thesis are the study for experimental procedures, including the analysis of uncertainties and error influences and propagation. The blockage effect has also been numerically investigated, checking that the classic Glauert correction is satisfactory. Also, classic fluid topology optimization, the double pipe problem, was applied using FVM in an external CFD code successfully.

For future works, it is interesting to validate the SH model by comparing it with experimental results: for this, it recommends to apply an SH treatment using a painting or some mechanical approach, since the turbulent condition of the propeller can be harsh for painting. The feasibility of conducting a controlled corrosion treatment to obtain a hydrophobic surface by electrolysis can be evaluated. It should take note of the price of propeller manufacturing and the complexity of defining electrolytic parameters.

The development in this work, in which topology optimization on the surface is applied, can be extrapolated to other applications. An example could be the roughness distribution, in order to change the transition point using Laminar-Turbulent model. In this example, inducing a transition may be interesting to control the cavitation inception, even if its performance is degraded, which is critical for submarine applications, for example. 


\section{References}

ANDERSON, J. Computational Fluid Dynamics: The Basis with Applications. [S.l.: s.n.], 1995. 547 p. ISBN 0-07-001685-2. Cited in page 26.

ASME. Standard for Verification and Validation in Computational Fluid Dynamics and Heat Transfer. [S.l.], 2009. 100 p. Available from: < https://www.asme.org/products/ codes-standards/v-v-20-2009-standard-verification-validation $>$. Cited 2 times in pages 64 and 66.

BAKKER, A. Lecture 5 - Solution Methods Applied Computational Fluid Dynamics. 2006. 45 p. Cited 2 times in pages 33 and 36.

BENDSØE, M. P.; KIKUCHI, N. Generating optimal topologies in structural design using a homogenization method. Computer Methods in Applied Mechanics and Engineering, v. 71, n. 2, p. 197-224, 11 1988. ISSN 00457825. Available from: $<$ http://linkinghub.elsevier.com/retrieve/pii/0045782588900862>. Cited 3 times in pages 21,37 , and 42 .

BIPM, I. et al. International vocabulary of metrology - Basic and general concepts and associated terms (VIM). JcGM, v. 200, p. 2012, 2012. Available from: $<$ https://www.bipm.org/utils/common/documents/jcgm/JCGM_200_2012.pdf >. Cited in page 136 .

BORRVALL, T.; PETERSSON, J. Topology optimization of fluids in Stokes flow. International Journal for Numerical Methods in Fluids, v. 41, n. 1, p. 77-107, 12003. ISSN 0271-2091. Available from: <http://doi.wiley.com/10.1002/fld.426>. Cited 5 times in pages $37,46,125,126$, and 129 .

BOUMAN, E. A. et al. State-of-the-art technologies, measures, and potential for reducing GHG emissions from shipping - A review. Transportation Research Part D: Transport and Environment, The Authors, v. 52, p. 408-421, 52017. ISSN 13619209. Available from: <http://dx.doi.org/10.1016/j.trd.2017.03.022https: //linkinghub.elsevier.com/retrieve/pii/S1361920916307015>. Cited in page 17.

BRENNEN, C. E. Cavitation and bubble dynamics. [s.n.], 1995. v. 9. 145-185 p. ISSN 1350-4177. ISBN 0195094093. Available from: <http://arjournals.annualreviews.org/doi/ abs/10.1146/annurev.fl.09.010177.001045> . Cited in page 31.

CARLTON, J. Marine Propellers and Propulsion. [S.1.]: Elsevier Ltd, 2012. ISSN 1098-6596. ISBN 978-07506-8150-6. Cited 2 times in pages 25 and 26.

CASTRO, D.; KIYONO, C.; SILVA, E. Design of radiative enclosures by using topology optimization. International Journal of Heat and Mass Transfer, Elsevier Ltd, v. 88, p. 880-890, 9 2015. ISSN 00179310. Available from: < http: //dx.doi.org/10.1016/j.ijheatmasstransfer.2015.04.077http://linkinghub.elsevier.com/ retrieve/pii/S0017931015004470>. Cited in page 21. 
CHENG, Y.-T.; RODAK, D. E. Is the lotus leaf superhydrophobic? Applied Physics Letters, v. 86, n. 14, p. 144101, 4 2005. ISSN 0003-6951. Available from: $<$ http://aip.scitation.org/doi/10.1063/1.1895487>. Cited in page 18.

CHO, S. C. et al. Surface modification of polyimide films, filter papers, and cotton clothes by HMDSO/toluene plasma at low pressure and its wettability. Current Applied Physics, Elsevier B.V., v. 9, n. 6, p. 1223-1226, 11 2009. ISSN 15671739. Available from: <http://dx.doi.org/10.1016/j.cap.2009.01.020http: //linkinghub.elsevier.com/retrieve/pii/S1567173909000339>. Cited in page 18.

DANiEllo, R. J.; WATERHOUSE, N. E.; ROTHSTEIN, J. P. Drag reduction in turbulent flows over superhydrophobic surfaces. Physics of Fluids, v. 21, n. 8, p. 085103, 8 2009. ISSN 1070-6631. Available from: <http://aip.scitation.org/doi/10.1063/1.3207885>. Cited 2 times in pages 19 and 33.

DANTAS, J. L. D. et al. Modernização do Túnel de Cavitação do Instituto de Pesquisas Tecnológicas de São Paulo. In: 25 Congresso Nacional de Transporte Aquaviário, Construção Naval e Offshore. Rio de Janeiro: SOBENA - Sociedade Brasileira de Engenharia Naval, 2014. v. 2014. Cited in page 132.

DILGEN, C. B. et al. Topology optimization of turbulent flows. Computer Methods in Applied Mechanics and Engineering, Elsevier B.V., v. 331, p. 363-393, 2018. ISSN 00457825. Available from: <https://doi.org/10.1016/j.cma.2017.11.029>. Cited in page 42.

Dolfin-adjoint. Topology optimisation of fluids in Stokes flow. 2019. Available from: < http://www.dolfin-adjoint.org/en/latest/documentation/stokes-topology/ stokes-topology.html>. Cited in page 129.

EVGRAFOV, A. The Limits of Porous Materials in the Topology Optimization of Stokes Flows. Applied Mathematics and Optimization, v. 52, n. 3, p. 263-277, 10 2005. ISSN 0095-4616. Available from: <http://link.springer.com/10.1007/s00245-005-0828-z>. Cited in page 37.

FU, Y.; YUAN, C.; BAI, X. A numerical study of drag reduction of superhydrophobic surfaces in shipping industry. In: 2017 4th International Conference on Transportation Information and Safety (ICTIS). IEEE, 2017. v. 0, n. 3, p. 99-103. ISBN 978-1-5386-0437-3. Available from: <http://ieeexplore.ieee.org/document/8047750/>. Cited in page 17.

GAO, L.; LIU, Y.; HU, H. An Experimental Investigation on the Dynamic Ice Accretion Process over the Surface of a Wind Turbine Blade Model. In: 9th AIAA Atmospheric and Space Environments Conference. Reston, Virginia: American Institute of Aeronautics and Astronautics, 2017. p. 1-16. ISBN 978-1-62410-496-1. Available from: $<$ https://arc.aiaa.org/doi/10.2514/6.2017-3582>. Cited in page 20.

GERSBORG-HANSEN, A.; BENDSØE, M. P.; SIGMUND, O. Topology optimization of heat conduction problems using the finite volume method. Structural and Multidisciplinary Optimization, v. 31, n. 4, p. 251-259, 4 2006. ISSN 1615-147X. Available from: $<$ http://link.springer.com/10.1007/s00158-005-0584-3>. Cited in page 21.

GERSBORG-HANSEN, A.; SIGMUND, O.; HABER, R. Topology optimization of channel flow problems. Structural and Multidisciplinary Optimization, v. 30, n. 3, p. 181-192, 92005. 
ISSN 1615-147X. Available from: <http://link.springer.com/10.1007/s00158-004-0508-7>. Cited 2 times in pages 37 and 42 .

GILES, M. B.; PIERCE, N. A. An Introduction to the Adjoint Approach to Design. Flow, Turbulence and Combustion, v. 65, n. 3/4, p. 393-415, 2000. ISSN 13866184. Available from: <http://link.springer.com/10.1023/A:1011430410075>. Cited in page 41.

GLAUERT, H. The Elements of Aerofoil and Airscrew Theory. Cambridge: Cambridge University Press, 1983. v. 236. 102 p. ISSN 00160032. ISBN 9780511574481.

Available from: <http://linkinghub.elsevier.com/retrieve/pii/S0016003243912141http: //ebooks.cambridge.org/ref/id/CBO9780511574481>. Cited in page 151.

GUEST, J. K.; PRÉVOST, J. H. Topology optimization of creeping fluid flows using a Darcy-Stokes finite element. International Journal for Numerical Methods in Engineering, v. 66, n. 3, p. 461-484, 4 2006. ISSN 0029-5981. Available from: $<$ http://doi.wiley.com/10.1002/nme.1560>. Cited in page 37 .

HAFTKA, R. T.; GÜRDAL, Z. Elements of structural optimization. 3rd rev. a. ed. Dordrecht: Kluwer Academic Publishers, 1992. Cited in page 39.

ITTC. ITTC - Recommended Procedures: Fresh Water and Seawater Properties. [S.1.], 2011. 1-45 p. Available from: <ittc.info/media/1215/75-02-01-03.pdf >. Cited in page 146.

ITTC. Specialist Committee on Energy Saving Methods. Wuxi, 2017. Available from: $<$ https://www.ittc.info/media/7827/20-sc-energy-saving-methods.pdf $>$. Cited in page 17.

JCGM, J. et al. Evaluation of measurement data - Guide to the ex pression of uncertainty in measurement. Bureau International des Poids et Mesures, 2008. Available from: <https://www.bipm.org/utils/common/documents/jcgm/JCGM_100_2008_E.pdf >. Cited in page 136.

JEONG, J.; HUSSAIN, F. On the identification of a vortex. Journal of Fluid Mechanics, v. 285, n. -1, p. 69, 2 1995. ISSN 0022-1120. Available from: < http: //www.journals.cambridge.org/abstract_S0022112095000462>. Cited in page 67 .

KATSUNO, E. T.; DANTAS, J. L. D. Investigação da metodologia para simulações de propulsores utilizando fluidodinâmica computacional. In: $26^{\circ}$ Congresso Nacional de Transporte Aquaviário, Construção Naval e Offshore. Rio de Janeiro: SOBENA, 2016. p. 1-8. Cited in page 59.

KATSUnO, E. T.; DANTAS, J. L. D. Analysis of the Blockage Effect on a Cavitation Tunnel Using CFD Tools. In: Volume 7B: Ocean Engineering. ASME, 2017. p. V07BT06A043. ISBN 978-0-7918-5774-8. Available from: <http://proceedings. asmedigitalcollection.asme.org/proceeding.aspx?doi=10.1115/OMAE2017-61545>. Cited in page 151.

KATSUnO, E. T.; DANTAS, J. L. D.; SILVA, E. C. N. Analysis of Hydrophobic Painting in Model-Scale Marine Propeller. In: Volume 7B: Ocean Engineering. Madrid: ASME, 2018. p. V07BT06A042. ISBN 978-0-7918-5127-2. Available from: < http://proceedings. asmedigitalcollection.asme.org/proceeding.aspx?doi=10.1115/OMAE2018-78209>. Cited in page 75 . 
KORKUT, E.; ATLAR, M. An experimental investigation of the effect of foul release coating application on performance, noise and cavitation characteristics of marine propellers. Ocean Engineering, Trondheim, Norway, v. 41, n. June, p. 1-12, 22012. ISSN 00298018. Available from: <http://linkinghub.elsevier.com/retrieve/pii/S0029801811002873>. Cited in page 17.

LAUNDER, B.; SPALDING, D. The numerical computation of turbulent flows. Computer Methods in Applied Mechanics and Engineering, v. 3, n. 2, p. 269-289, 3 1974. ISSN 00457825. Available from: < http://linkinghub.elsevier.com/retrieve/pii/ 0045782574900292>. Cited in page 29.

LIU, Y.; CHEN, X.; XIN, J. H. Can superhydrophobic surfaces repel hot water? Journal of Materials Chemistry, v. 19, n. 31, p. 5602, 2009. ISSN 0959-9428. Available from: <http://xlink.rsc.org/?DOI=b822168e > . Cited 2 times in pages 18 and 19 .

LONGVA, T.; EIDE, M. S.; SKJONG, R. Determining a required energy efficiency design index level for new ships based on a cost-effectiveness criterion. Maritime Policy E Management, v. 37, n. 2, p. 129-143, 3 2010. ISSN 0308-8839. Available from: <http://www.tandfonline.com/doi/abs/10.1080/03088830903533759>. Cited in page 17.

MÄKIHARJU, S. A.; CECCIO, S. L. On multi-point gas injection to form an air layer for frictional drag reduction. Ocean Engineering, v. 147, n. October 2017, p. 206-214, 1 2018. ISSN 00298018. Available from: <http://linkinghub.elsevier.com/retrieve/pii/ S0029801817306492>. Cited in page 17.

MENTER, F. R. Two-equation eddy-viscosity turbulence models for engineering applications. AIAA Journal, v. 32, n. 8, p. 1598-1605, 8 1994. ISSN 0001-1452. Available from: <http://arc.aiaa.org/doi/10.2514/3.12149>. Cited in page 30.

MIN, T.; KIM, J. Effects of hydrophobic surface on skin-friction drag. Physics of Fluids, v. 16, n. 7, p. L55-L58, 7 2004. ISSN 1070-6631. Available from:

$<$ http://aip.scitation.org/doi/10.1063/1.1755723>. Cited 3 times in pages 19, 33, and 77 .

MUTTON, R. et al. Drag prevention coatings for marine propellers. In: 2nd International Symposium on Seawater Drag Reduction. Busan, Korea: [s.n.], 2005. p. 23-26. Cited in page 17 .

MUZENSKI, S.; FLORES-VIVIAN, I.; SOBOLEV, K. Hydrophobic engineered cementitious composites for highway applications. Cement and Concrete Composites, Elsevier Ltd, v. 57, p. 68-74, 3 2015. ISSN 09589465. Available from: < http: //dx.doi.org/10.1016/j.cemconcomp.2014.12.009http://linkinghub.elsevier.com/retrieve/ pii/S0958946514002339>. Cited in page 18.

OTHMER, C. A continuous adjoint formulation for the computation of topological and surface sensitivities of ducted flows. International Journal for Numerical Methods in Fluids, v. 58, n. 8, p. 861-877, 11 2008. ISSN 02712091. Available from: $<$ http://doi.wiley.com/10.1002/fld.1770>. Cited in page 37 .

OTHMER, C. Adjoint methods for car aerodynamics. Journal of Mathematics in Industry, v. 4, n. 1, p. 6, 2014. ISSN 2190-5983. Available from: <http: //mathematicsinindustry.springeropen.com/articles/10.1186/2190-5983-4-6>. Cited in page 40 . 
OU, J.; PEROT, B.; ROTHSTEIN, J. P. Laminar drag reduction in microchannels using ultrahydrophobic surfaces. Physics of Fluids, v. 16, n. 12, p. 4635-4643, 12 2004. ISSN 1070-6631. Available from: <http://aip.scitation.org/doi/10.1063/1.1812011>. Cited 2 times in pages 18 and 32 .

PARK, J. et al. Design and fabrication of a superhydrophobic glass surface with micro-network of nanopillars. Journal of Colloid and Interface Science, Elsevier Inc., v. 360, n. 1, p. 272-279, 8 2011. ISSN 00219797. Available from: <http://dx.doi.org/10. 1016/j.jcis.2011.04.047http://linkinghub.elsevier.com/retrieve/pii/S0021979711004644>. Cited in page 18.

PATANKAR, S. Numerical heat transfer and fluid flow. [S.l.: s.n.], 1980. 1-197 p. ISBN 0-07-048740-5. Cited in page 33.

PINGEN, G.; MAUTE, K. Optimal design for non-Newtonian flows using a topology optimization approach. Computers 85 Mathematics with Applications, Elsevier Ltd, v. 59, n. 7, p. 2340-2350, 4 2010. ISSN 08981221. Available from: <http://dx.doi.org/10.1016/ j.camwa.2009.08.044http://linkinghub.elsevier.com/retrieve/pii/S0898122109006336>. Cited in page 42.

PIRONNEAU, O. On optimum profiles in Stokes flow. Journal of Fluid Mechanics, v. 59, n. 01, p. 117, 6 1973. ISSN 0022-1120. Available from: <http://www.journals.cambridge. org/abstract_S002211207300145X>. Cited in page 40.

POPE, S. B. Turbulent Flows. [S.l.]: IOP Publishing, 2000. v. 1. 771 p. ISSN 1468-5248. ISBN 9780511840531. Cited in page 28.

REUTHER, J. et al. Aerodynamic shape optimization of supersonic aircraft configurations via an adjoint formulation on distributed memory parallel computers. Computers \& Fluids, v. 28, n. 4-5, p. 675-700, 5 1999. ISSN 00457930. Available from: $<$ http://linkinghub.elsevier.com/retrieve/pii/S0045793098000504>. Cited in page 40.

ROMERO, J.; SILVA, E. A topology optimization approach applied to laminar flow machine rotor design. Computer Methods in Applied Mechanics and Engineering, Elsevier B.V., v. 279, p. 268-300, 9 2014. ISSN 00457825. Available from: <http://dx.doi.org/10.1016/j. cma.2014.06.029http://linkinghub.elsevier.com/retrieve/pii/S0045782514002151>. Cited 2 times in pages 21 and 42 .

ROWIN, W. A.; HOU, J.; GHAEMI, S. Inner and outer layer turbulence over a superhydrophobic surface with low roughness level at low Reynolds number. Physics of Fluids, v. 29, n. 9, p. 095106, 9 2017. ISSN 1070-6631. Available from: <http://aip.scitation.org/doi/10.1063/1.5004398>. Cited in page 19.

ROZVANY, G. I. N.; ZHOU, M.; BIRKER, T. Generalized shape optimization without homogenization. Structural Optimization, v. 4, n. 3-4, p. 250-252, 9 1992. ISSN 0934-4373. Available from: <http://link.springer.com/10.1007/BF01742754>. Cited in page 41.

SÁNCHEZ-CAJA, A.; SIPILÄ, T. P.; PYLKKÄNEN, J. V. Simulation of Viscous Flow around a Ducted Propeller with Rudder Using Different RANS-Based Approaches. First International Symposium on Marine Propulsion, n. June, p. 1-8, 2009. Cited in page 60. 
SASAKI, N. Boundary Layer Control of Twin Skeg Hull Form with Reaction Podded Propulsion. In: Second International Symposium on Marine Propulsors. Hamburg: [s.n.], 2011. Cited in page 17.

SATO, Y. et al. An Optimum Design Method for a Thermal-Fluid Device Incorporating Multiobjective Topology Optimization With an Adaptive Weighting Scheme. Journal of Mechanical Design, v. 140, n. 3, p. 031402, 2018. ISSN 1050-0472. Available from: $<$ http://mechanicaldesign.asmedigitalcollection.asme.org/article.aspx?doi=10.1115/1. 4038209>. Cited in page 52.

SEO, J.; MANI, A. On the scaling of the slip velocity in turbulent flows over superhydrophobic surfaces. Physics of Fluids, v. 28, n. 2, p. 025110, 2 2016. ISSN 1070-6631. Available from: <http://aip.scitation.org/doi/10.1063/1.4941769>. Cited 2 times in pages 19 and 33.

SHIH, T.-H. et al. A new k-epsilon eddy viscosity model for high reynolds number turbulent flows. Computers \& Fluids, v. 24, n. 3, p. 227-238, 3 1995. ISSN 00457930. Available from: <http://linkinghub.elsevier.com/retrieve/pii/004579309400032T>. Cited in page 29 .

Siemens. STAR-CCM+ Documentation - version 12.02. 2017. Cited 8 times in pages 28, $29,31,36,46,48,55$, and 69 .

SLOTNICK, J. et al. CFD Vision 2030 Study: A Path to Revolutionary Computational Aerosciences. [S.l.], 2014. 1-73 p. Available from: <http://ntrs.nasa.gov/search.jsp?R= 20140003093>. Cited 2 times in pages 22 and 101.

SPALART, P.; ALLMARAS, S. A one-equation turbulence model for aerodynamic flows. In: 30th Aerospace Sciences Meeting and Exhibit. Reston, Virigina: American Institute of Aeronautics and Astronautics, 1992. ISBN 1111111111. ISSN 00341223. Available from: $<$ http://arc.aiaa.org/doi/10.2514/6.1992-439>. Cited in page 30.

VERSTEEG, H. K.; MALALASEKERA, W. An introduction to computational fluid dynamics: the finite volume method. [S.l.: s.n.], 2007. ISBN 978-0-13-127498-3. Cited 5 times in pages $26,28,30,33$, and 35 .

VORONOV, R. S.; PAPAVASSILIOU, D. V.; LEE, L. L. Boundary slip and wetting properties of interfaces: Correlation of the contact angle with the slip length. The Journal of Chemical Physics, v. 124, n. 20, p. 204701, 5 2006. ISSN 0021-9606. Available from: $<$ http://aip.scitation.org/doi/10.1063/1.2194019>. Cited in page 18.

VORONOV, R. S.; PAPAVASSILIOU, D. V.; LEE, L. L. Slip length and contact angle over hydrophobic surfaces. Chemical Physics Letters, v. 441, n. 4-6, p. 273-276, 6 2007. ISSN 00092614. Available from: < http://linkinghub.elsevier.com/retrieve/pii/ S0009261407005866>. Cited in page 18.

VORONOV, R. S.; PAPAVASSILIOU, D. V.; LEE, L. L. Review of Fluid Slip over Superhydrophobic Surfaces and Its Dependence on the Contact Angle. Industrial \& Engineering Chemistry Research, v. 47, n. 8, p. 2455-2477, 4 2008. ISSN 0888-5885. Available from: <http://pubs.acs.org/doi/abs/10.1021/ie0712941>. Cited in page 18. 
WÄCHTER, A.; BIEGLER, L. T. On the implementation of an interior-point filter line-search algorithm for large-scale nonlinear programming. Mathematical Programming, v. 106, n. 1, p. 25-57, 3 2006. ISSN 0025-5610. Available from: $<$ http://link.springer.com/10.1007/s10107-004-0559-y>. Cited in page 56.

WANG, C. et al. Applying periodic boundary conditions to predict open water propeller performance. Journal of Marine Science and Application, v. 9, n. 3, p. 262-267, 2010. ISSN 16719433. Cited in page 60.

WANG, L. et al. A study of the mechanical and chemical durability of Ultra-Ever Dry Superhydrophobic coating on low carbon steel surface. Colloids and Surfaces A: Physicochemical and Engineering Aspects, v. 497, p. 16-27, 2016. ISSN 18734359. Cited in page 19.

WILCOX, D. C. Turbulence Modelling for CFD. [S.l.: s.n.], 1993. v. 1. ISBN 0-9636051-0-0. Cited in page 30 . 

Appendix 



\section{APPENDIX A - Fluid Topology Optimization of Double Pipe}

The objective of this chapter is to verify if the numerical implementation, as described in Sec. 5 by using Star-CCM+ and IPOPT, is appropriately working, checking not only in results but also if the time spent is coherent with the case complexity. To perform this, it is interesting to choose a standard fluid topology optimization in the literature to compare results. It is chosen the double pipe problem with an aspect ratio of 1.5, as described in Borrvall and Petersson (2003).

\section{A.1 Problem description}

Given a rectangular domain $1.0 \times 1.5$, the objective is to distribute material such that minimize the dissipated power in the fluid. The domain and some boundary conditions are shown in Fig. 86: there are two inlets with a given parabolic velocity distribution and two outlets, also with a given parabolic velocity distribution. The other surfaces are treated as walls, without velocity on it.

Figure 86 - Design domain dimensions and boundary conditions

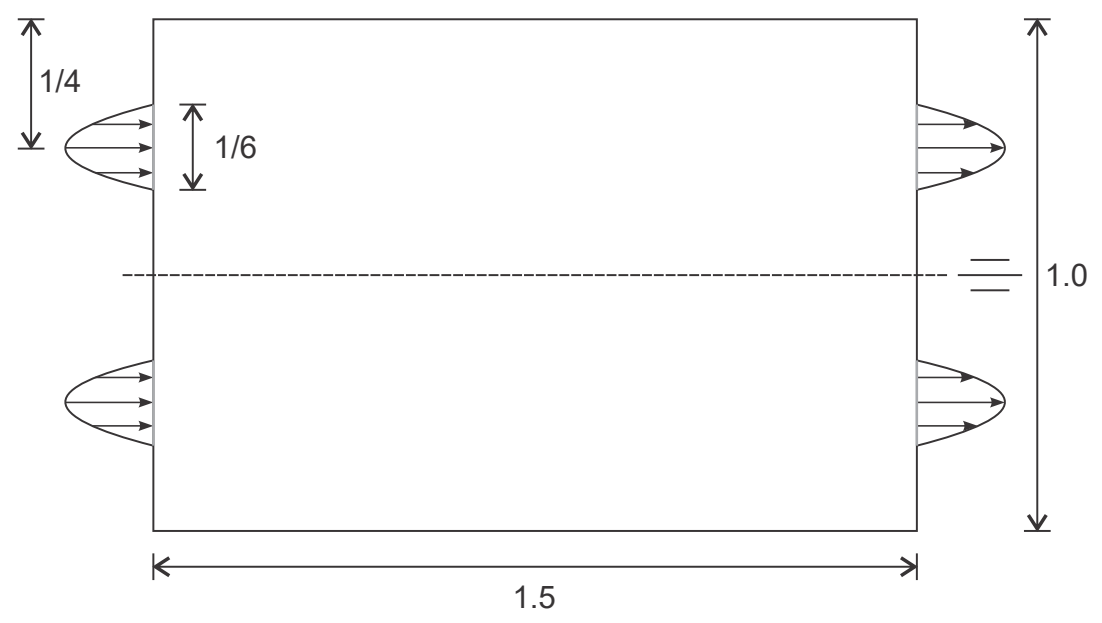

Source: Author 


\section{A.2 Stokes flow formulation}

The fluid flow is modeled as Stokes flow. The governing fluid equations with velocity Dirichlet conditions (Brinkman model) are given by:

$$
\begin{aligned}
& 0=\nabla \cdot \mathbf{u} \\
& 0=-\nabla p+\mu \nabla^{2} \mathbf{u}-\kappa \mathbf{u}
\end{aligned}
$$

There is no direct way to simulate Stokes flow in Star-CCM+, but the laminar flow model is available. To turn around, a volume source term is applied in such that cancels with the convective term. Since the convective term is given by a surface integral, it can be transformed to volume source term from divergence theorem:

$$
\int_{\Omega}(\nabla \cdot \mathbf{F}) d \Omega=\oint_{\partial \Omega}(\mathbf{F} \cdot \mathbf{n}) d s
$$

in which the divergence of a tensor is:

$$
\nabla \cdot \mathbf{F}=\mathbf{e}_{j} \frac{\partial F_{i j}}{\partial x_{i}}
$$

If the tensor $\mathbf{F}$ is:

$$
\nabla \cdot \rho \mathbf{u u}=\rho\left[\nabla \cdot v_{x} \mathbf{u}, \nabla \cdot v_{y} \mathbf{u}, \nabla \cdot v_{z} \mathbf{u},\right]^{T}
$$

then, by applying the divergence theorem, it will be equal to $\oint_{\partial \Omega} \rho \mathbf{u u} \cdot d \mathbf{s}$ and will cancel with convective term in the LHS. In this way, the Navier-Stokes formulation in Star-CCM+ is converted to the Stokes flow condition.

\section{A.3 Material model}

Different from the SIMP material model used for hydrophobic cases, in this problem it is used the material model presented in Borrvall and Petersson (2003), given by:

$$
\kappa(\hat{\rho})=\bar{\kappa}+(\underline{\kappa}-\bar{\kappa}) \hat{\rho} \frac{1+q}{\hat{\rho}+q}
$$

The parameter $q>0$ is the penalization value. The smaller, the more convex the function $\kappa$. The adopted values are $\underline{\kappa}=2.5 \mu / 100^{2}$ and $\bar{\kappa}=2.5 \mu / 0.01^{2}$. Density $\rho$ and viscosity $\mu$ are unit value, respectively. 


\section{A.4 Optimization formulation}

The optimization formulation is to minimize the dissipated power in the fluid $\Phi$ and to control constraints on available fluid volume up to $30 \%$ of the domain:

$$
\begin{array}{|ll}
\underset{\hat{\rho}}{\operatorname{Min}} & C=\Phi=\int_{\Omega}\left[\kappa(\hat{\rho}) \mathbf{u} \cdot \mathbf{u}+\mu\|\mathbf{D}\|^{2}\right] d \Omega \\
\text { s.t. } & \hat{g}(\hat{\rho})=\frac{\int_{\Omega} \hat{\rho} d \Omega}{\int_{\Omega} d \Omega} \leq 0.3 \\
& 0 \leq \hat{\rho} \leq 1
\end{array}
$$

\section{A.5 Sensitivity comparison between Adjoint and Finite Difference methods}

Before performing the topology optimization, the sensitivity through the adjoint method is compared with FDM. The purpose is to check if the derivation of the adjoint formulation is correct and close to FDM model, which is more computationally expensive. For the study case $\hat{\rho}=0.5$ in whole domain and $q=10$. The sensitivity through the adjoint method is made by applying Eq. (4.6). Based on the current problem, some derivatives are defined below:

$$
\begin{gathered}
\frac{\partial \kappa}{\partial \hat{\rho}}=\frac{q(\underline{\kappa}-\bar{\kappa})(q+1)}{(\hat{\rho}+q)^{2}} \\
\frac{\partial J}{\partial \hat{\rho}}=V \mathbf{u} \cdot \mathbf{u} \\
\frac{\partial \mathbf{f}}{\partial \kappa}=\left[0,-\mathbf{u}^{T}\right]^{T}
\end{gathered}
$$

To obtain the sensitivity by FDM, it uses 4 points and adopting $\Delta \hat{\rho}=0.025$. The derivative through FDM is:

$$
\begin{aligned}
\left.\frac{d \phi}{d \hat{\rho}}\right|_{\mathrm{FDM}}=\frac{1}{\Delta \hat{\rho}} & {\left[\frac{1}{12} \phi\left(\hat{\rho}_{i}+2 \Delta \hat{\rho}\right)-\frac{2}{3} \phi\left(\hat{\rho}_{i}+\Delta \hat{\rho}\right)+\right.} \\
& \left.+\frac{2}{3} \phi\left(\hat{\rho}_{i}-\Delta \hat{\rho}\right)-\frac{1}{12} \phi\left(\hat{\rho}_{i}-2 \Delta \hat{\rho}\right)\right]
\end{aligned}
$$

The comparison between the two results, adopting the same color scale, is shown in Fig. 87. As can be observed, the results are close to each other.

To enhance the difference, Fig. 88 shows the absolute difference between sensitivity using Adjoint Method and FDM. As can be noted, the difference compared with the 
Figure 87 - Comparative between sensitivity obtained by using Adjoint Method (left) and Finite Difference Method (right)

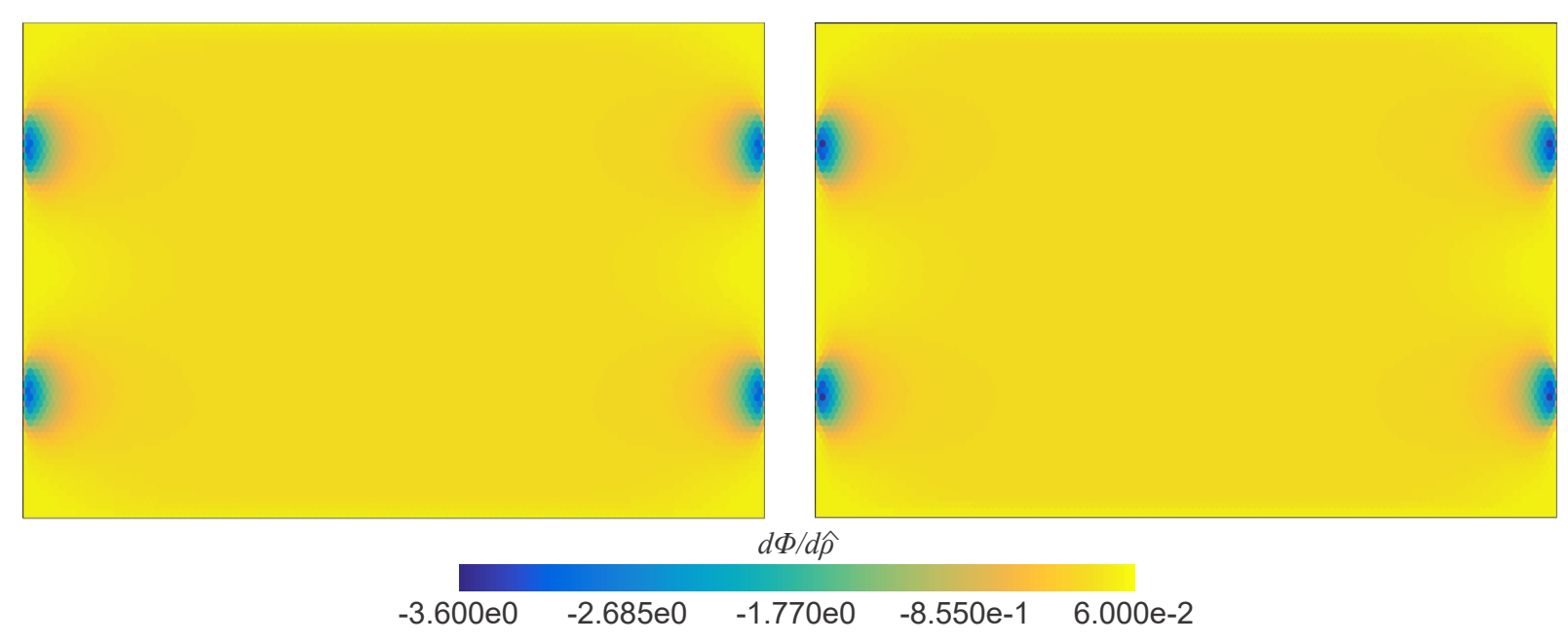

Source: Author

absolute value is minimal, showing that two methods can produce similar sensitivity. The results endorse the use of adjoint methods as a fast method to obtain sensitivities.

Figure 88 - Absolute difference between sensitivity by using Adjoint and Finite Difference Method

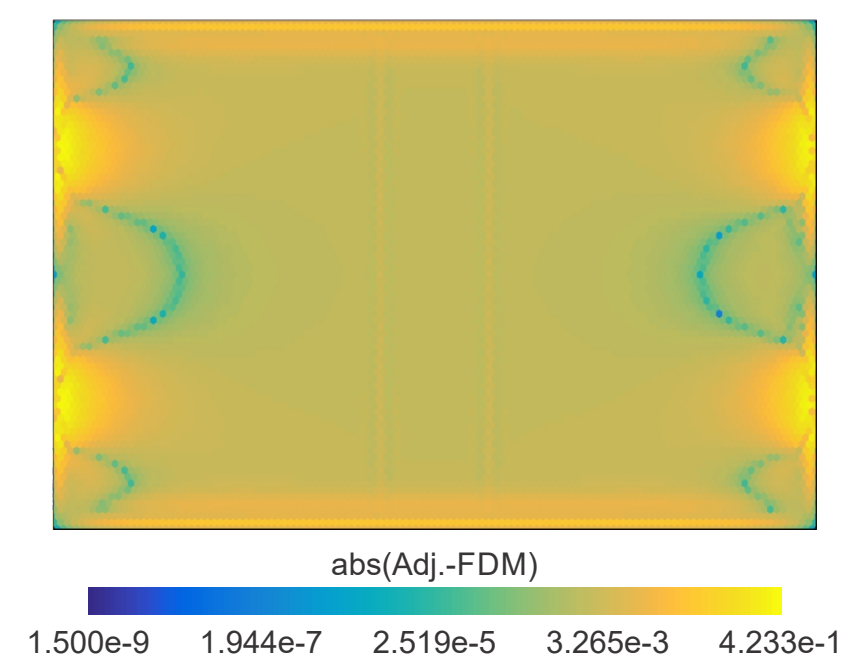

Source: Author

\section{A.6 Results and Comparison}

Figure 89 shows the results of topology optimization by adopting two penalty factor $q$. Both results show the same geometry, but the higher penalty factor presents more extremized values of $\hat{\rho}$. 
Figure 89 - Results of Double Pipe Problem in Stokes flow for $q=0.1$ (left); and for $q=$ 1.0 (right). Black regions indicate solid (low $\hat{\rho}$ ).
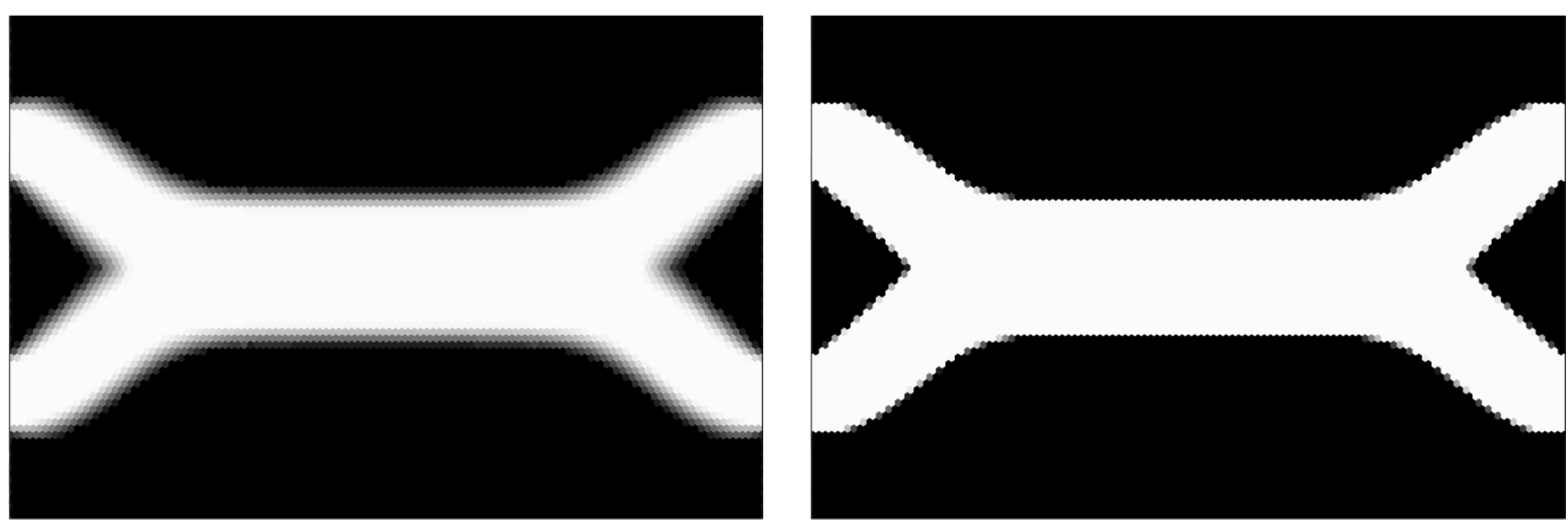

Source: Author

As a comparison, Fig. 90 shows the results obtained using FEniCS and the results from original work.

Figure 90 - Double Pipe in Stokes flow using FEniCS/dolfin-adjoint (left) and from Borrvall and Petersson (2003) (right).

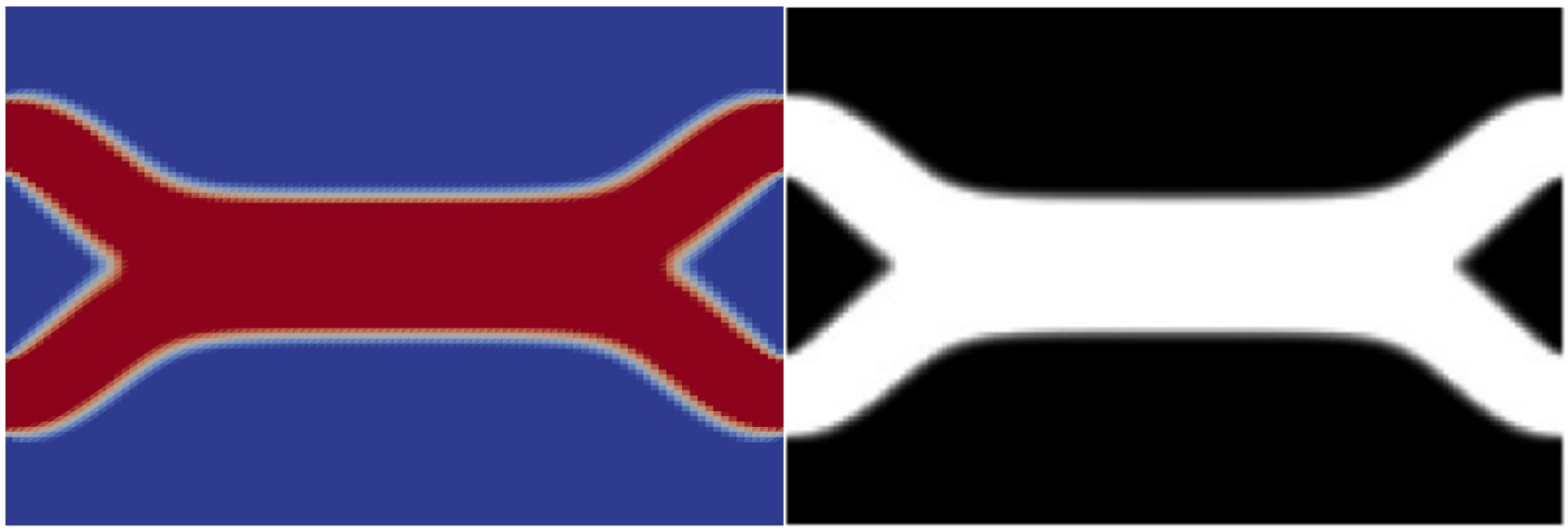

Source: Dolfin-adjoint (2019)

The visual comparison of obtained geometry, its format, and Y format connecting inputs and outputs are similar in all methods.

In conclusion, although this fluid topology optimization is not the same as the hydrophobic painting distribution, it shows that the numerical implementation proposed results in expected geometry with also similar computation time. Moreover, it shows that the numerical implementation is flexible and can be adapted to other types of fluid topology optimization, with the advantage of having an already-done fluid software. 



\section{APPENDIX B - Experimental procedures - IPT's Cavitation Tunnel Verification}

\section{B.1 Introduction}

Cavitation Tunnels are a circulating water channel that allows the control over the water velocity, rotation rate in the propeller, and hydrostatic pressure inside it, for controlled cavitation experiments. The most common application of the cavitation tunnels, in which it is the objective of this work, is to analyze the hydrodynamic of propellers. In this case, the propellers are installed on an axis connected to a dynamometer, serving to measure the hydrodynamic forces and torques generated by these propellers in multiple operating conditions, defined by the rotation of the propeller, the water velocity (or the advance velocity) and the hydrostatic pressure. The IPT's cavitation tunnel is shown in Fig. 91.

Figure 91 - Superior view of IPT's Cavitation Tunnel (left); and a test in cavitating condition of $\mathrm{H} 1$ propeller (right)
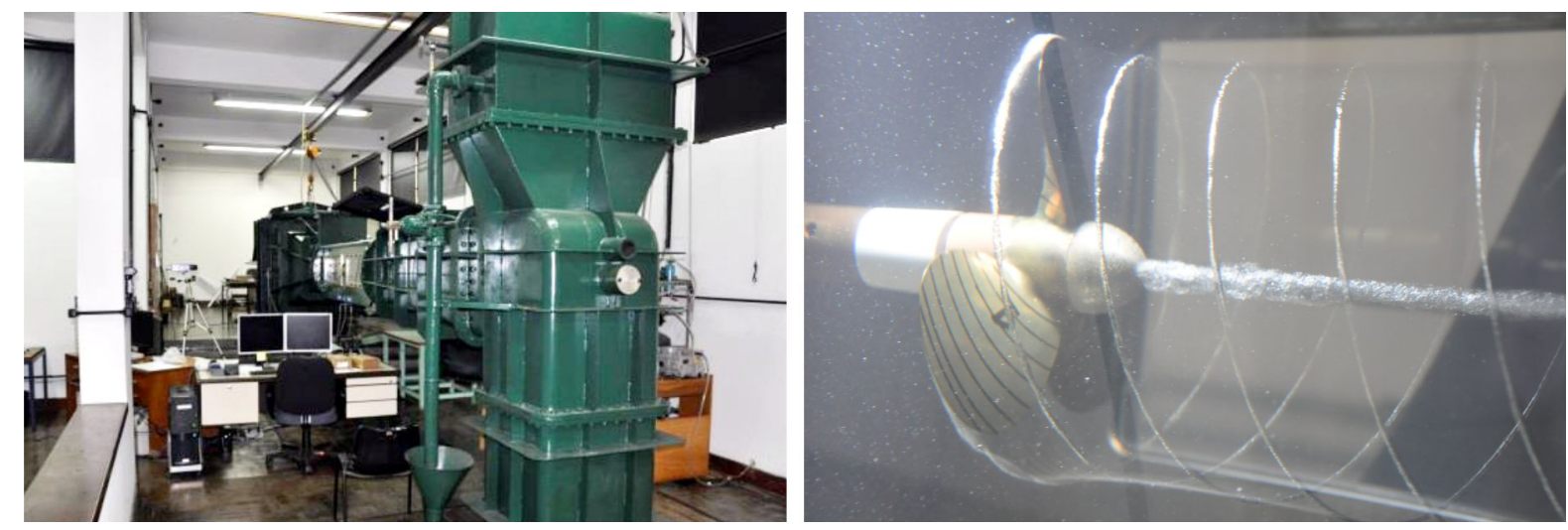

Source: Author

It has a square cross-sectional of $0.50 \mathrm{~m}$. Measurements of thrust and torque are performed by loads cells connected to a drive motor and propeller axis by two pendulum systems. This system allows obtaining a full scale of 20 to $1000 \mathrm{~N}$, maintaining a linearity error of $0.05 \%$. Due to the modularity of mechanism, it is possible to conduct tests with low and high loads with good accuracy.

The flow velocity at the test section is measured indirectly, using a pressure transducer to measure the difference between two points in a Venturi-like shaped duct. Therefore, the average flow velocity is calculated by applying Bernoulli and Continuity 
equations in these measured points. More detail of IPT's Cavitation Tunnel and its equipment can be found in Dantas et al. (2014).

In a typical configuration of a test in a Cavitation Tunnel, hydrodynamic characteristics of a propeller are evaluated: imposing a condition in function of an advance velocity, rotation and hydrostatic pressure, it is obtained the thrust and required torque. Figure 92 schematically shows the equipment for data acquisition and the processes for obtaining the value of characteristics in a Cavitation Tunnel test.

Figure 92 - Schematics of Cavitation Tunnel instrumentation

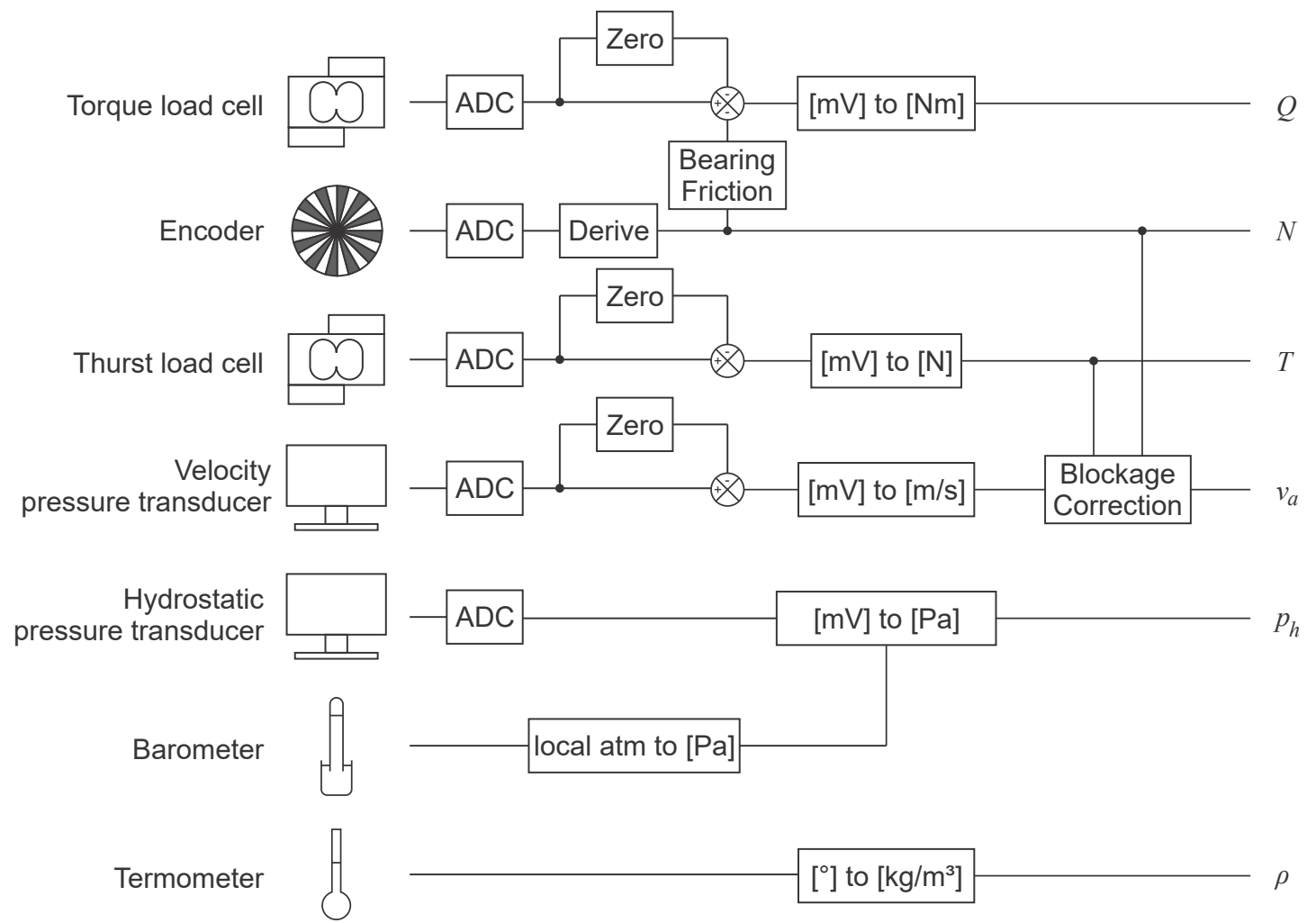

Source: Author

Based on the schema of Fig. 92, this chapter is distributed to verify the main procedures in the instrumentation of the Cavitation Tunnel. Firstly, a theoretical review of uncertainty in linear regression is presented in Sec. B.2. The sources of uncertainties are discussed in Sec. B.4. Then, uncertainties of main coefficients for propeller are shown in Sec. B.3, in which each subsequent section is indicated in order to show how to compose the full formulation of the uncertainty of a specific coefficient. The turbulence intensity, used as an input of CFD simulations, is detailed in Sec. B.13. Blockage correction is presented in an exclusive section, in Appendix C. 


\section{B.2 Theoretical Review of Uncertainty in Linear Regression}

The linear regression of one variable is given by:

$$
\hat{y}(x)=\hat{\beta}_{0}+\hat{\beta}_{1} x
$$

In general, a linear regression for calibration is done to use its inverse function, i.e., obtain the $x$ value given the $y_{0}$ value. Thus, the inverse function is given by:

$$
\hat{x}\left(y_{0}\right)=\frac{y_{0}-\hat{\beta}_{0}}{\hat{\beta}_{1}}
$$

A confidence interval on the slope $\beta_{1}$, on intercept $\beta_{0}$ and on the mean response $\hat{x}$ at value of $y=y_{0}$ is given by:

$$
\begin{gathered}
\hat{\beta}_{1}-t(\alpha / 2, N-2) \sigma_{\beta_{1}} \leq \hat{\beta}_{1} \leq \hat{\beta}_{1}+t(\alpha / 2, N-2) \sigma_{\beta_{1}} \\
\hat{\beta}_{0}-t(\alpha / 2, N-2) \sigma_{\beta_{0}} \leq \hat{\beta}_{0} \leq \hat{\beta}_{0}+t(\alpha / 2, N-2) \sigma_{\beta_{0}} \\
\hat{x}\left(y_{0}\right)-t(\alpha / 2, N-2) \sigma_{x} \leq \hat{x}\left(y_{0}\right) \leq \hat{x}\left(y_{0}\right)+t(\alpha / 2, N-2) \sigma_{x}
\end{gathered}
$$

In Eq. (B.3), Eq. (B.4) and Eq. (B.5), $t(\alpha / 2, N-2)$ is the Student's $t$-distribution for a confidence of $\alpha$ and for $N$ degrees of freedom; $\sigma_{\beta_{0}}$, standard deviation of $\beta_{0} ; \sigma_{\beta_{1}}$, standard deviation of $\beta_{1}$; and $\sigma_{x}$, standard deviation of mean value of $\hat{x}$ at $y=y_{0}$. Before showing the definitions of standard deviations, some useful properties are presented.

The error sum of squares is given by:

$$
S S_{E}=\sum_{i=1}^{N}\left(y_{i}-\hat{y}\left(x_{i}\right)\right)^{2}
$$

The sum of the squares of the difference between each $x$ and the mean $\bar{x}$ value is given by:

$$
S_{x x}=\sum_{i=1}^{N}\left(x_{i}-\bar{x}\right)^{2}
$$

Similarly, the sum of the squares of the difference between each $y$ and the mean $\bar{y}$ value is given by:

$$
S_{y y}=\sum_{i=1}^{N}\left(y_{i}-\bar{y}\right)^{2}
$$


The standard deviation of $y(x)$ is given by:

$$
\sigma_{y, x}=\sqrt{\frac{S S_{E}}{N-2}}
$$

Therefore, the standard deviation of intercept and slope are given, respectively, by:

$$
\begin{gathered}
\sigma_{\beta_{0}}=\sigma_{y, x} \sqrt{\frac{1}{N}+\frac{\bar{x}^{2}}{S_{x x}}} \\
\sigma_{\beta_{1}}=\sigma_{y, x} \frac{1}{\sqrt{S_{x x}}}
\end{gathered}
$$

Based on the Eq. (B.2), it is possible to formulate the standard deviation of $\hat{x}$, given by Eq. (B.12).

$$
\sigma_{x}=\frac{1}{\hat{\beta}_{1}} \sqrt{\sigma_{y_{0}}^{2}+\sigma_{\beta_{1}} \hat{x}^{2}\left(y_{0}\right)+2 \hat{x}\left(y_{0}\right) \operatorname{cov}\left(\hat{\beta}_{0}, \hat{\beta}_{1}\right)+\sigma_{\beta_{0}}^{2}}
$$

Defining the standard deviation of $y_{0}$ based on its $M$ measurements, the equation results in:

$$
\sigma_{y_{0}}=\frac{\sigma_{y, x}}{\sqrt{M}}
$$

Therefore, the standard deviation of the inverse function of linear regression is given by:

$$
\begin{gathered}
\sigma_{x}=\frac{\sigma_{y, x}}{\hat{\beta}_{1}} \sqrt{1+\frac{1}{N}+\frac{\left(\hat{x}\left(y_{0}\right)-\bar{x}\right)^{2}}{S_{x x}}} \\
\sigma_{y}=\sigma_{y, x} \sqrt{1+\frac{1}{N}+\frac{\left(x_{0}-\bar{x}\right)^{2}}{S_{x x}}}
\end{gathered}
$$

The coefficient of determination $R^{2}$ is given by:

$$
R^{2}=1-\frac{S S_{E}}{S_{y y}}
$$




\section{B.3 Propeller coefficients}

The main coefficients for open-water propeller are shown in Sec. 2.1. The uncertainty composition and the section to calculate each term of its uncertainty are shown below:

$$
\begin{gathered}
\left(\frac{u_{J}}{J}\right)^{2}=\underbrace{\left(\frac{u_{v_{a}}}{v_{a}}\right)^{2}}_{\text {Sec. B.7.3 }}+\underbrace{\left(\frac{u_{n}}{n}\right)^{2}}_{\text {Sec. B.8 }}+\underbrace{\left(\frac{u_{D_{p}}}{D_{p}}\right)^{2}}_{\approx 0} \\
\left(\frac{u_{K T}}{K T}\right)^{2}=\underbrace{\left(\frac{u_{T}}{T}\right)^{2}}_{\text {Sec. B.6.1 }}+\underbrace{\left(\frac{u_{\rho}}{\rho}\right)^{2}}_{\text {Sec. B.9 }}+\underbrace{\left(2 \frac{u_{n}}{n}\right)^{2}}_{\text {Sec. B.8 }}+\underbrace{\left(4 \frac{u_{D_{p}}}{D_{p}}\right)^{2}}_{\approx 0} \\
\left(\frac{u_{K Q}}{K Q}\right)^{2}=\underbrace{\left(\frac{u_{Q}}{Q}\right)^{2}}_{\text {Sec. B.6.2 }}+\underbrace{\left(\frac{u_{\rho}}{\rho}\right)^{2}}_{\text {Sec. B.9 }}+\underbrace{\left(2 \frac{u_{n}}{n}\right)^{2}}_{\text {Sec. B.8 }}+\underbrace{\left(5 \frac{u_{D_{p}}}{D_{p}}\right)^{2}}_{\approx 0} \\
\left(\frac{u_{\eta}}{\eta}\right)^{2}=\underbrace{\left(\frac{u_{T}}{T}\right)^{2}}_{\text {Sec. B.6.1 }}+\underbrace{\left(\frac{u_{v_{a}}}{v_{a}}\right)^{2}}_{\text {Sec. B.7.3 }}+\underbrace{\left(\frac{u_{Q}}{Q}\right)^{2}}_{\text {Sec. B.6.2 }}+\underbrace{\left(\frac{u_{n}}{n}\right)^{2}}_{\text {Sec. B.8 }}
\end{gathered}
$$

\section{B.4 Source of uncertainty}

For each tested case from batch of tests, the following variable must be acquired. All these variables have uncertainty formed by a square-sum of two terms: measurement and resolution.

Table 7 - Measurements obtained for each case tested in the Cavitation Tunnel

\begin{tabular}{cl}
\hline Variable & Description \\
\hline$U_{T}$ & Voltage of thrust load cell \\
$U_{Q}$ & Voltage of torque load cell \\
$n$ & Rotation \\
$i_{v}$ & Current of velocity pressure transducer \\
$p_{r}$ & Hydrostatic pressure ratio \\
$\theta$ & Temperature \\
$p_{a t m}$ & Atmospheric pressure \\
\hline
\end{tabular}

For all batch of tests, the following constants in Tab 8 must be obtained, in which it is assumed to be equal for all cases in a batch of tests.

Each expression used to obtain a variable of interest may have an addition of uncertainty due to the method, determined by experience. 
Table 8 - Values that must be obtained for a batch of tests in the Cavitation Tunnel

\begin{tabular}{cl}
\hline Variable & Description \\
\hline$D_{p}$ & Propeller diameter \\
$A_{t}$ & Tunnel's cross section \\
$g$ & Gravity \\
$b_{T}$ & Lever ratio \\
$b_{Q}$ & Lever arm \\
$\hat{\beta}_{,}$ & Linear regression of thrust load cell \\
$\hat{\beta}_{, Q}$ & Linear regression of torque load cell \\
$\hat{\beta}_{, B}$ & Linear regression of bearing friction \\
$\hat{\beta}_{, v}$ & Linear regression of velocity \\
\hline
\end{tabular}

\section{B.5 Measurement and Resolution uncertainties}

The measurement uncertainty $u_{\text {disp }}$ is treated as a type A methodology for uncertainty. According to the Vocabulary in Metrology (BIPM et al., 2012) "type A Uncertainty is the evaluation of a component of measurement uncertainty by a statistical analysis of measured quantity values obtained under defined measurement conditions."

Thus, it is assumed that digital data from data acquisition systems represent several measurements and theses measurements have a Gaussian behavior, being given by:

$$
u_{d i s p}=s
$$

in which $s$ is the standard deviation of the sample.

An example of data acquisition is shown in Fig. 93, which shows the acquired thrust and torque data, as well as the mean and standard deviation of this sample.

To compute the resolution uncertainty, it follows JCGM et al. (2008), which adopts a rectangular distribution when using digitally acquired systems, such as load cells and pressure transducers, Eq. (B.22); or triangular distribution, when measuring using analog equipment, usually performed through the eye of a human, such as reading a thermometer or barometer, Eq. (B.23).

$$
\begin{gathered}
u_{\text {disc }}=\frac{2 a}{\sqrt{12}}=\frac{U_{\text {range }}}{2^{n_{\text {bits }}}} \frac{1}{\sqrt{12}} \\
u_{\text {disc }}=\frac{2 a}{\sqrt{24}}
\end{gathered}
$$


Figure 93 - Example of 4000 samples acquired with the thrust and torque load cells
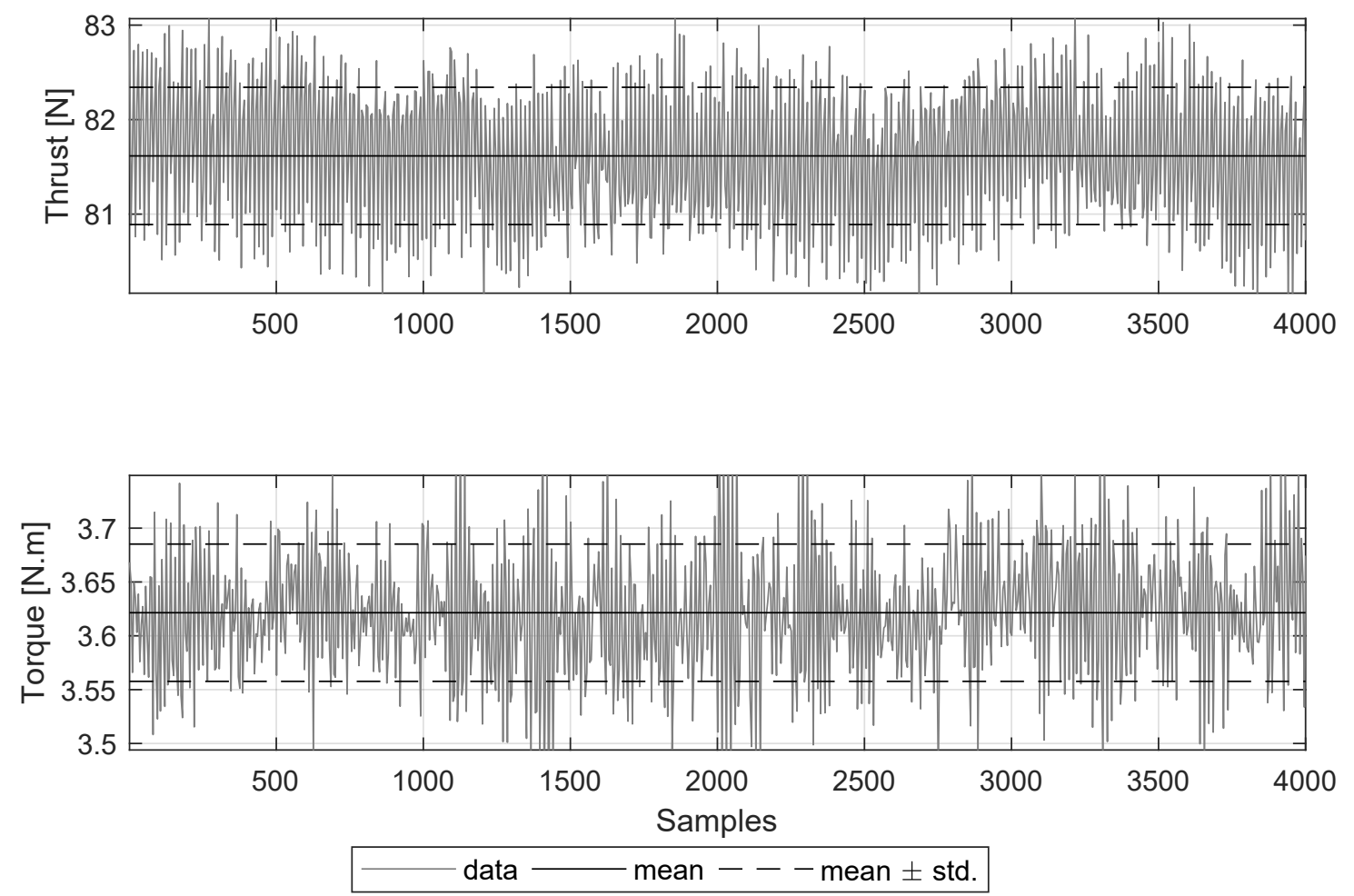

Source: Author

\section{B.6 Force and Moment Verification}

\section{B.6.1 Thrust Expression and Uncertainty Expansion}

The thrust measurement is initiated by reading the thrust load cell voltage. This

value is converted to its corresponding mass, using the coefficients $\hat{\beta}_{0, T}$ and $\hat{\beta}_{1, T}$. The mass value is converted to force by multiplying by $g$, obtaining the force acting on the load cell. However, due to a ratio of forces between the point of measurement of the load cell and propeller thrust, seen in Fig. 94, the value must be multiplied by a lever ratio $b_{T}$.

The equations that summarize the procedure and its respective uncertainty propagation are expressed by:

$$
T=b_{T}\left(\frac{U_{T}-\hat{\beta}_{0, T}}{\hat{\beta}_{1, T}}\right) g
$$


Figure 94 - Lever arm schema.

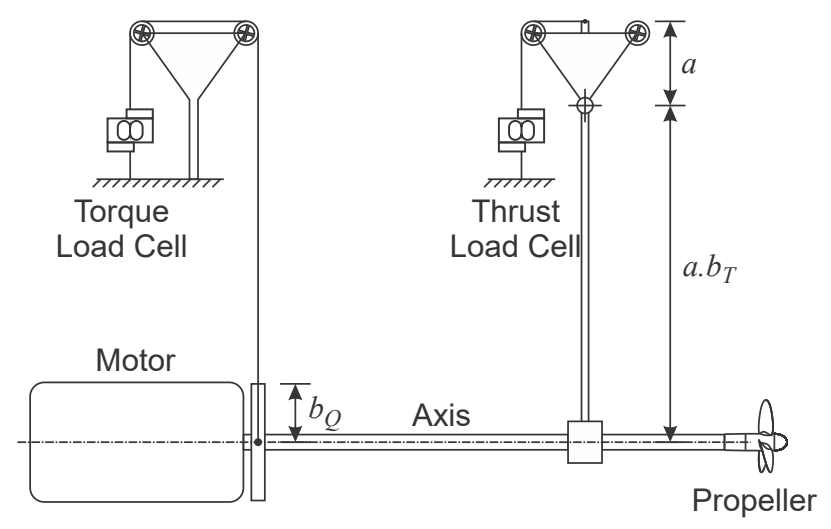

Source: Author

$$
\begin{aligned}
u_{T}^{2}=\left(\frac{T}{b_{T}}\right)^{2} \underbrace{u_{b_{T}}^{2}}_{\text {Sec. B.12 }}+\left(\frac{b_{T} g}{\hat{\beta}_{1, T}}\right)^{2} u_{U_{T}}^{2}+\left(\frac{b_{T} g}{\hat{\beta}_{1, T}}\right)^{2} \underbrace{u_{\hat{\beta}_{0, T}}^{2}}_{\text {Sec. B.6.3 }}+ \\
\left(\frac{T}{\hat{\beta}_{1, T}}\right)^{2} \underbrace{u_{\hat{\beta}_{1, T}}^{2}}_{\text {Sec. B.6.3 }}+\left(\frac{T}{g}\right)^{2} \underbrace{u_{g}^{2}}_{\text {Sec. B.11 }}+u_{m e t h o d, T}^{2}
\end{aligned}
$$

Two types of uncertainty composes $u_{U_{T}}$ : measurement and resolution:

$$
u_{U_{T}}^{2}=u_{d i s p, T}^{2}+u_{d i s c r, T}^{2}
$$

The relation between the strength applied on a load cell and its output voltage is discussed in Sec. B.6.3.

\section{B.6.2 Torque Expression and Uncertainty Expansion}

Torque measurement is initiated by reading the torque load cell voltage. However, unlike the procedure for thrust, this value is subtracted by the linearization of the voltage value due to the bearing friction of the Cavitation Tunnel axis, using the coefficients $\hat{\beta}_{0, B}$ and $\hat{\beta}_{1, B}$. The description of bearing friction is discussed in Sec. B.6.4. The equivalent mass value is multiplied by the gravity $g$ and, finally, by arm ratio $b_{Q}$, resulting in the propeller torque. Figure 94 shows schematically the dimension $b_{Q}$, which corresponds to the radius of the disc that is coupled to the motor. Also, a metal ribbon that attaches with the loading cell is tangentially coupled to this disc.

The equations that summarize the procedure and its respective uncertainty propa- 
gation are expressed by:

$$
\begin{gathered}
Q=b_{Q}\left(\frac{U_{Q}-\hat{\beta}_{0, Q}-\hat{\beta}_{0, B}-\hat{\beta}_{1, B} n}{\hat{\beta}_{1, Q}}\right) g \\
u_{Q}^{2}=\left(\frac{Q}{b_{Q}}\right)^{2} \underbrace{u_{b_{Q}}^{2}}_{\text {Sec. B.6.3 }}+\left(\frac{b_{Q} g}{\hat{\beta}_{1, Q}}\right)^{2} u_{U_{Q}}^{2}+\left(\frac{b_{Q} g}{\hat{\beta}_{1, Q}}\right)^{2} \underbrace{u_{\hat{\beta}_{0, Q}}^{2}}_{\text {Sec. B.6.3 }}+ \\
\left(\frac{b_{Q} g}{\hat{\beta}_{1, Q}}\right)^{2} \underbrace{u_{\hat{\beta}_{0, B}}^{2}}_{\text {Sec. B.6.4 }}+\left(\frac{b_{Q} g n}{\hat{\beta}_{1, Q}}\right)^{2} \underbrace{u_{\hat{\beta}_{1, B}}^{2}}_{\text {Sec. B.6.4 }}+\left(\frac{b_{Q} g \hat{\beta}_{1, B}}{\hat{\beta}_{1, Q}}\right)^{2} \underbrace{u_{n}^{2}}_{\text {Sec. B.8 }}+ \\
\underbrace{u_{g}^{2}}_{\text {Sec. B.11 }}+\left(\frac{Q}{\hat{\beta}_{1, Q}}\right)^{2} \underbrace{u_{\hat{\beta}_{1, Q}}^{2}}_{\text {Sec. B.6.3 }}+u_{\text {method, } Q}^{2}
\end{gathered}
$$

Two types composes $u_{U_{T}}$ : dispersion and discretization:

$$
u_{U_{Q}}^{2}=u_{d i s p, Q}^{2}+u_{d i s c r, Q}^{2}
$$

The same methodology used in the thrust load cell was applied to obtain the linear relation between the strength and output voltage and it is described in the next section.

\section{B.6.3 Load Cells}

To obtain the relation between the strength applied on a load cell and the output voltage, 13 masses were weighed in weighing scale with a $0.0001 \mathrm{~kg}$ resolution. The masses were added gradually, and then gradually removed while the output voltage was acquired. The obtained values and a linear interpolation of load cell used to measure the thrust and torque are presented in Fig. 95. The confidence intervals are not represented, once the intervals are too small to be represented graphically.

Table 9 shows the regression expression of the thrust load cell and Tab. 10, torque load cell.

Table 9 - Linear Regression inverse expression of Thrust Load Cell

\begin{tabular}{cl}
\hline Description & Value \\
\hline Expression & $m_{T}(U)=10.295681 U+0.133316$ \\
$R^{2}$ & 0.999952 \\
$u_{\hat{\beta}_{0, T}}$ & 0.001820 \\
$u_{\hat{\beta}_{1, T}}$ & 0.000155 \\
\hline
\end{tabular}


Figure 95 - Observed value of thrust (left) and torque (right) load cells

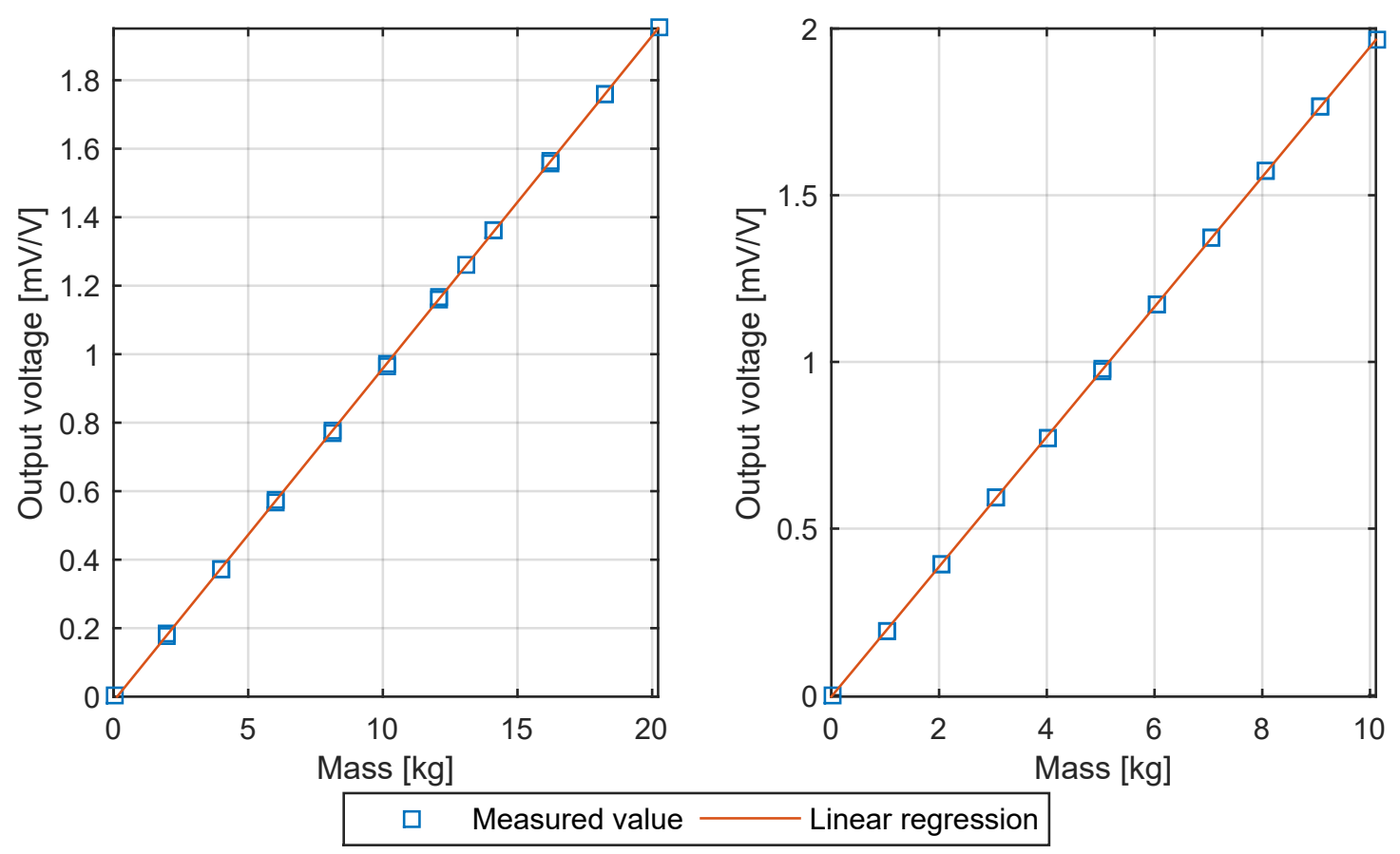

Source: Author

Table 10 - Linear Regression inverse expression of Torque Load Cell

\begin{tabular}{cl}
\hline Description & Value \\
\hline Expression & $m_{Q}(U)=5.131371 U+0.020765$ \\
$R^{2}$ & 0.999996 \\
$u_{\hat{\beta}_{0, Q}}$ & 0.0005179 \\
$u_{\hat{\beta}_{1, Q}}$ & 0.0000887 \\
\hline
\end{tabular}

\section{B.6.4 Bearing Friction}

The rotational movement of the axis implies in bearing friction, which increases the required torque. In order to eliminate this contribution, a test with an object without a low hydrodynamic body and the same weight as the propeller was performed for several rotational speed. In this way, it is expected that the torque acquired is only due to the bearing friction.

The obtained values, linear interpolation and confidence intervals are presented in Fig. 96.

Table 11 shows the regression expression of the bearing friction in the function of the rotation (in RPM). 
Figure 96 - Observed value for torque load cell

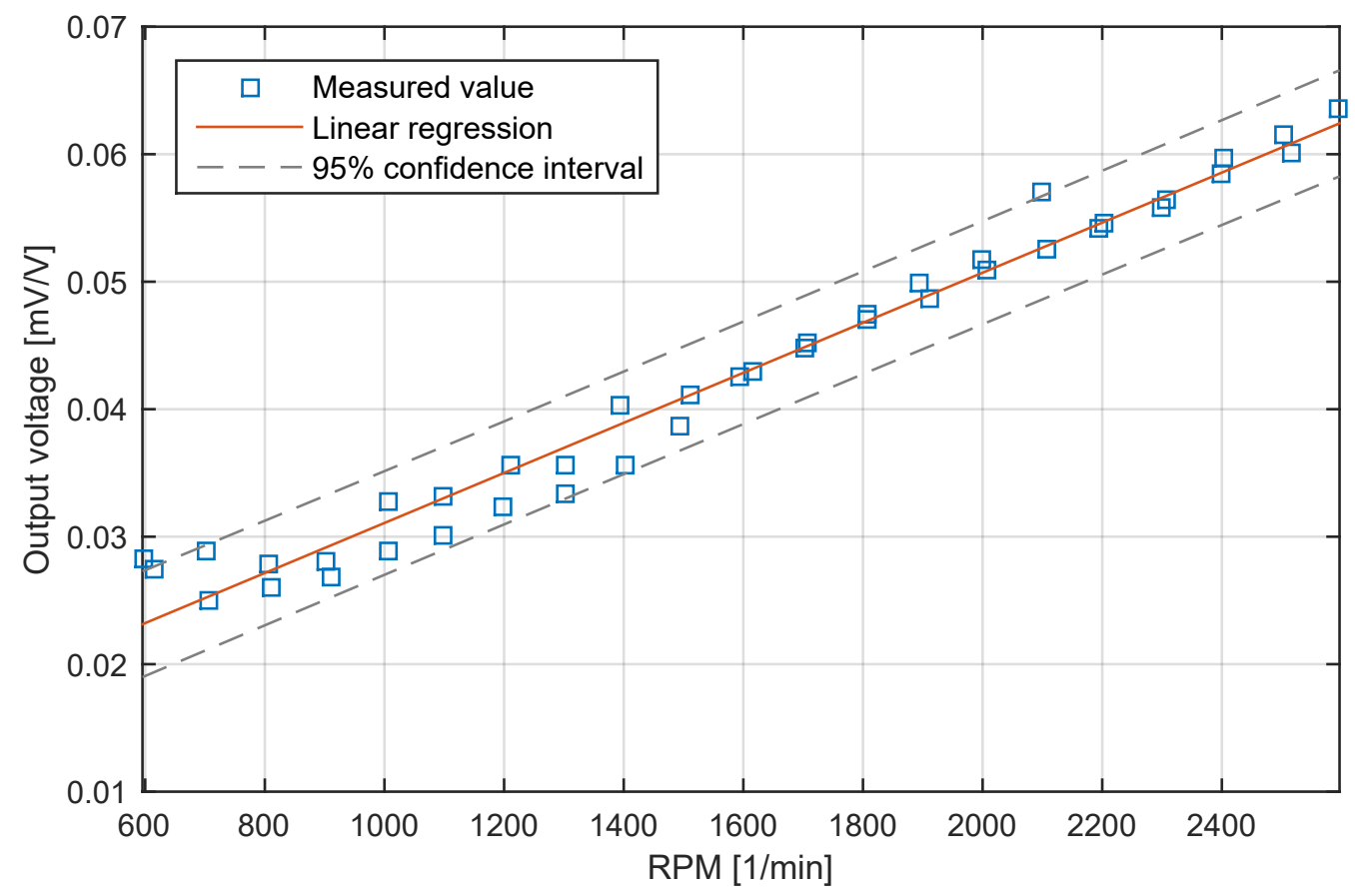

Source: Author

Table 11 - Linear Regression expression of Bearing Friction

\begin{tabular}{cl}
\hline Description & Value \\
\hline Expression & $U(N)=0.000020 N+0.011445$ \\
$R^{2}$ & 0.97351 \\
$u_{\hat{\beta}_{0, B}}$ & 0.0008744 \\
$u_{\hat{\beta}_{1, B}}$ & 0.0000311 \\
\hline
\end{tabular}

\section{B.7 Advance Velocity Verification}

\section{B.7.1 Upstream Velocity Expression and Uncertainty Expansion}

The measurement of flow velocity in the upstream section is performed indirectly using differential pressure transducers. These transducers measure the pressure difference $\Delta p$ between points in the large and narrow section that is geometrically similar to a Venturi tube, as it can be noted in Fig. 97. Note that we are calling the upstream velocity as the velocity in the narrow section.

Being $A_{r}$ the area ratio between large and narrow section, the mean upstream velocity is given by:

$$
v^{\prime}=k_{p}^{\prime} \sqrt{\frac{2 \Delta p}{\rho\left(1-A_{r}^{2}\right)}}=k_{p} \frac{1}{\sqrt{\rho}} \sqrt{\Delta p}
$$


Figure 97 - Cavitation Tunnel Geometry before the test section, showing the hoses where the pressures are taken at upstream and downstream of the tunnel (left) and its shape (right)
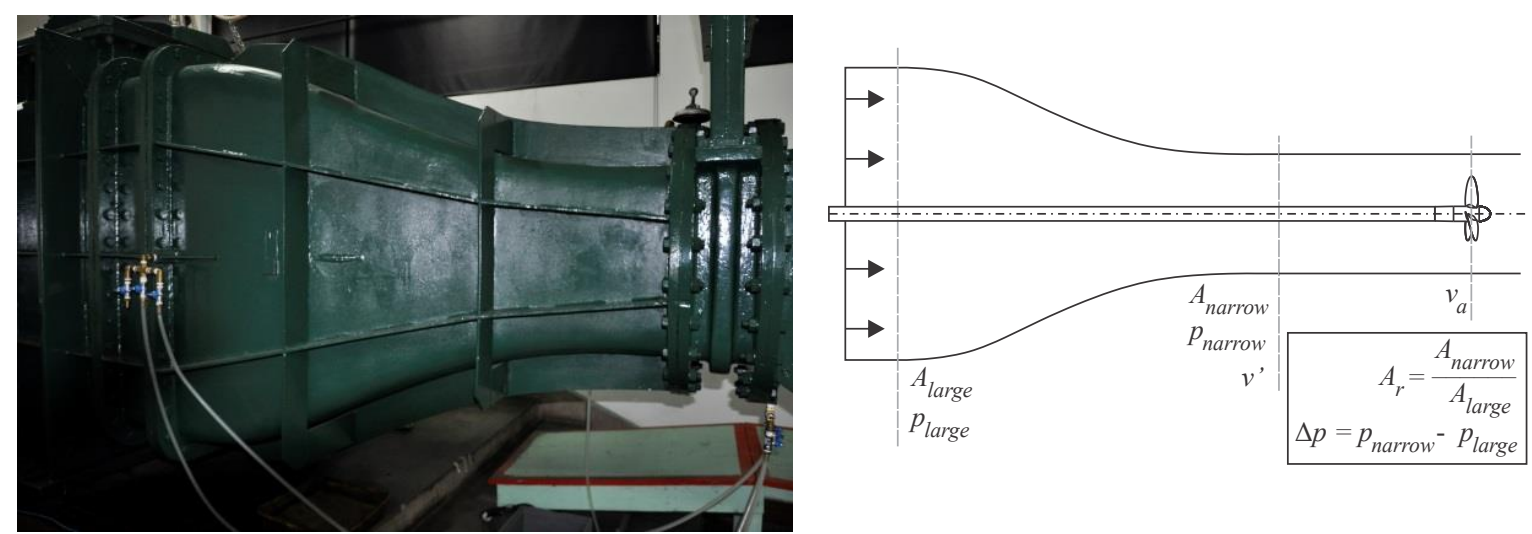

Source: Author

It can be observed that the pressure difference is proportional to the square of the upstream velocity. This variable is again attached to the angular coefficient of the linear regression of the pressure, i.e. $\hat{\beta}_{1, v}=k_{p} \hat{\beta}_{1, v}^{\prime}$. This procedure is adopted because the method to obtain linear regression coefficients also considers the attachment of $k_{p}$.

Therefore, the upstream velocity and its propagation of uncertainty are given by:

$$
\begin{gathered}
v^{\prime}=k_{p} \sqrt{\frac{i_{v}-\hat{\beta}_{0, v}}{\rho \hat{\beta}_{1, v}^{\prime}}}=\sqrt{\frac{i_{v}-\hat{\beta}_{0, v}}{\rho \hat{\beta}_{1, v}}} \\
u_{v^{\prime}}^{2}=\left(\frac{k_{p}^{2}}{2 \rho \hat{\beta}_{1, v} v^{\prime}}\right)^{2} u_{i_{v}}^{2}+\left(\frac{k_{p}^{2}}{2 \rho \hat{\beta}_{1, v} v^{\prime}}\right)^{2} u_{\hat{\beta}_{0, v}}^{2}+ \\
\left(\frac{v^{\prime}}{2 \hat{\beta}_{1, v}}\right)^{2} u_{\hat{\beta}_{1, v}}^{2}+\left(\frac{v^{\prime}}{2 \rho}\right)^{2} u_{\rho}^{2}+u_{\text {method, } v^{\prime}}^{2}
\end{gathered}
$$

Two types composes $u_{i_{v}}$ : measurement and resultion uncertainties:

$$
u_{i_{v}}^{2}=u_{d i s p, v}^{2}+u_{d i s c r, v}^{2}
$$

\section{B.7.2 Pitot Tube Velocity Measurement}

To obtain a correlation between pressure difference and mean upstream velocity, an S-type Pitot tube connected with a digital pressure transducer, both previously verified and calibrated, was used. Figure 98 shows the Pitot tube in the measurement and an inner view of the tunnel.

It is assumed that the tunnel is a perfect rounded square section with horizontal and vertical symmetries. Therefore, only the vertical direction is acquired. The tip of the 
Figure 98 - S-type Pitot tube (left); Pitot tube installed on the top of test section (center); and the apparatus inside the tunnel (right)
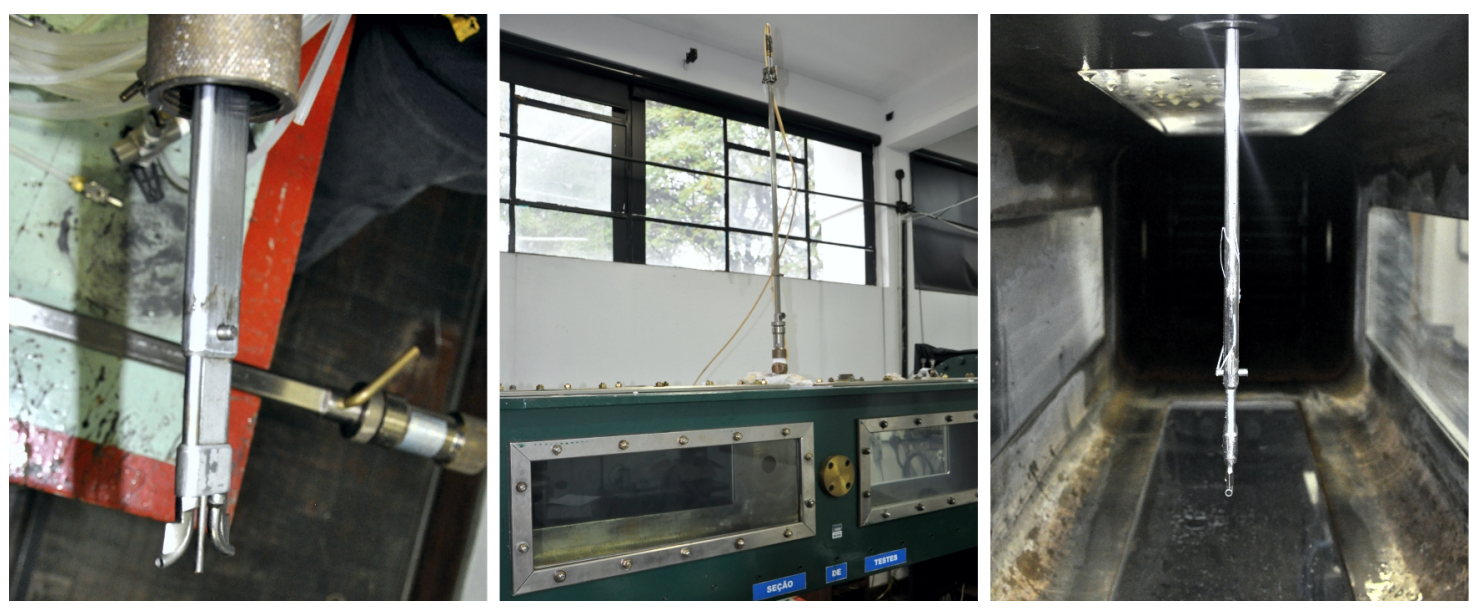

Source: Author

Pitot tube was placed in 11 different vertical positions of the test section. The Pitot was gradually moved up and down inside the cavitation Tunnel, so each position was acquired twice. The velocity in function of the vertical distance to the top wall is then obtained. It can be interpreted as the local velocity in function of a wall distance, assumption valid up to the half of Cavitation Tunnel. Hence, it is possible to obtain the expected velocity in the whole cross-section as a function of wall distance. Figure 99 shows the combination of information to obtain the local velocity in cross-section.

The data obtained with the Pitot tube are interpolated, and the relation between velocity and wall distance is calculated. Based on the wall distance of each region of the tunnel cross-section, the estimative of the velocity field in the whole cross-section is done. The mean advance velocity is the surface integral of velocity and area, divided by the total cross-sectional area of the tunnel.

By considering the relation of pressure and velocity, given by Eq. (B.30), the linear relation between the output current of the pressure transducer $i$ and the squared velocity $v^{2}$ is given by:

$$
i=\hat{\beta}_{0}+\frac{\hat{\beta}_{1}}{\left(C k_{p}\right)^{2}} v^{2}
$$

Figure 100 shows the linear regression with a $95 \%$ confidence interval of its inverse function, i.e., output current to squared velocity, and Tab. 12, the regression expression.

\section{B.7.3 Advance Velocity verification}

The upstream velocity is not the one that actually reaches the propeller and must be corrected due to blockage effects. The ratio between the equivalent advance velocity 
Figure 99 - Obtained values with Pitot tube (left-top), wall distance of each part of cross section (left-bottom) and the expected velocity (right)

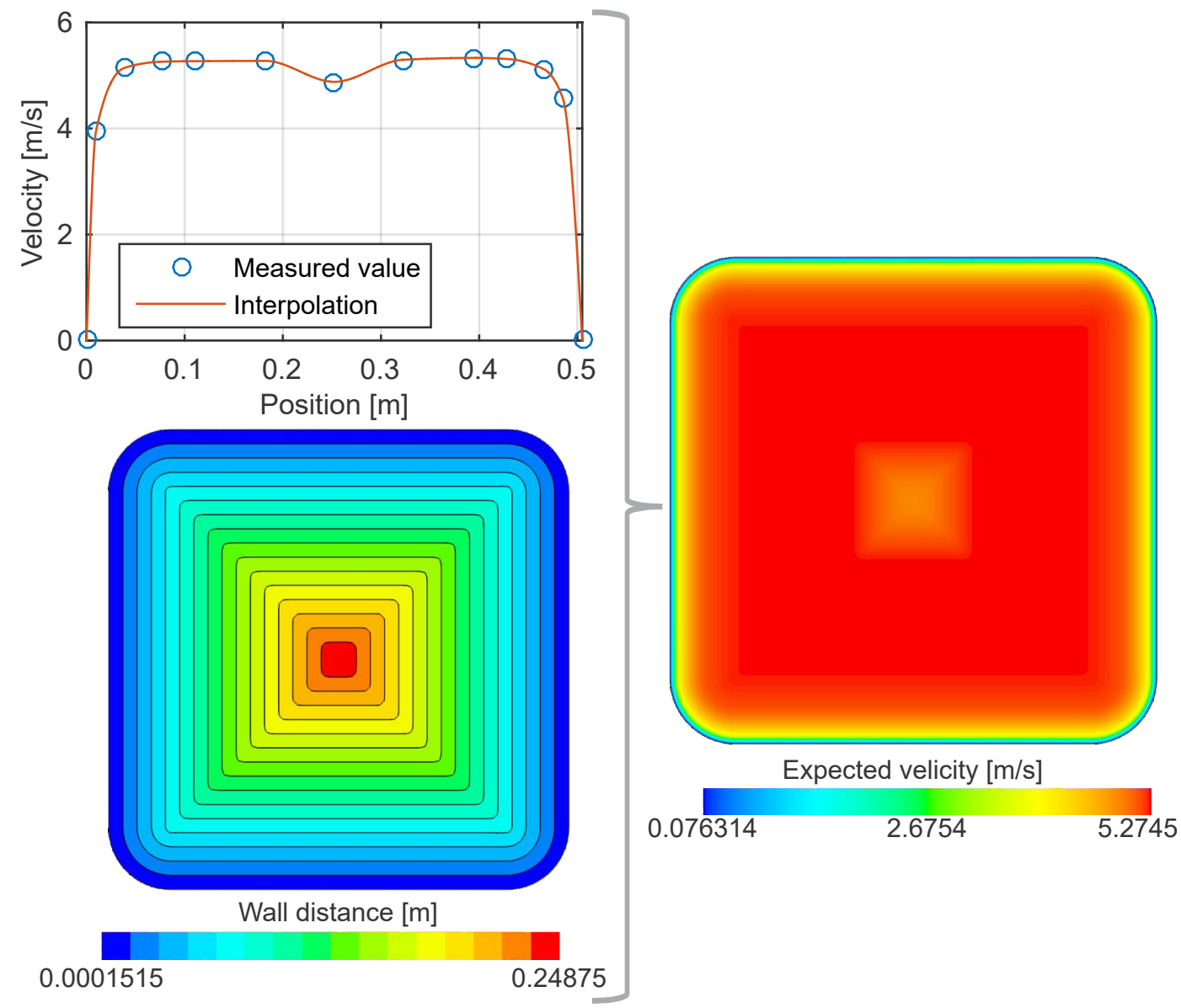

Source: Author

Table 12 - Linear Regression expression of Pressure Transducers

\begin{tabular}{cl}
\hline Description & Value \\
\hline Expression & $i\left(v^{2}\right)=0.144443 v^{2}+4.030234$ \\
$R^{2}$ & 0.99986 \\
$u_{\hat{\beta}_{0, v^{2}}}$ & 0.0113705 \\
$u_{\hat{\beta}_{1, v^{2}}}$ & 0.0008444 \\
\hline
\end{tabular}

and the upstream velocity is given by $\lambda$. The expression and its uncertainty are given by:

$$
\begin{gathered}
v_{a}=\lambda v^{\prime} \\
\left(\frac{u_{v_{a}}}{v_{a}}\right)^{2}=\underbrace{\left(\frac{u_{\lambda}}{\lambda}\right)^{2}}_{\text {Sec. B.7.4 }}+\underbrace{\left(\frac{u_{v^{\prime}}}{v^{\prime}}\right)^{2}}_{\text {Sec. B.7.1 }}
\end{gathered}
$$


Figure 100 - Observed value for thrust load cell

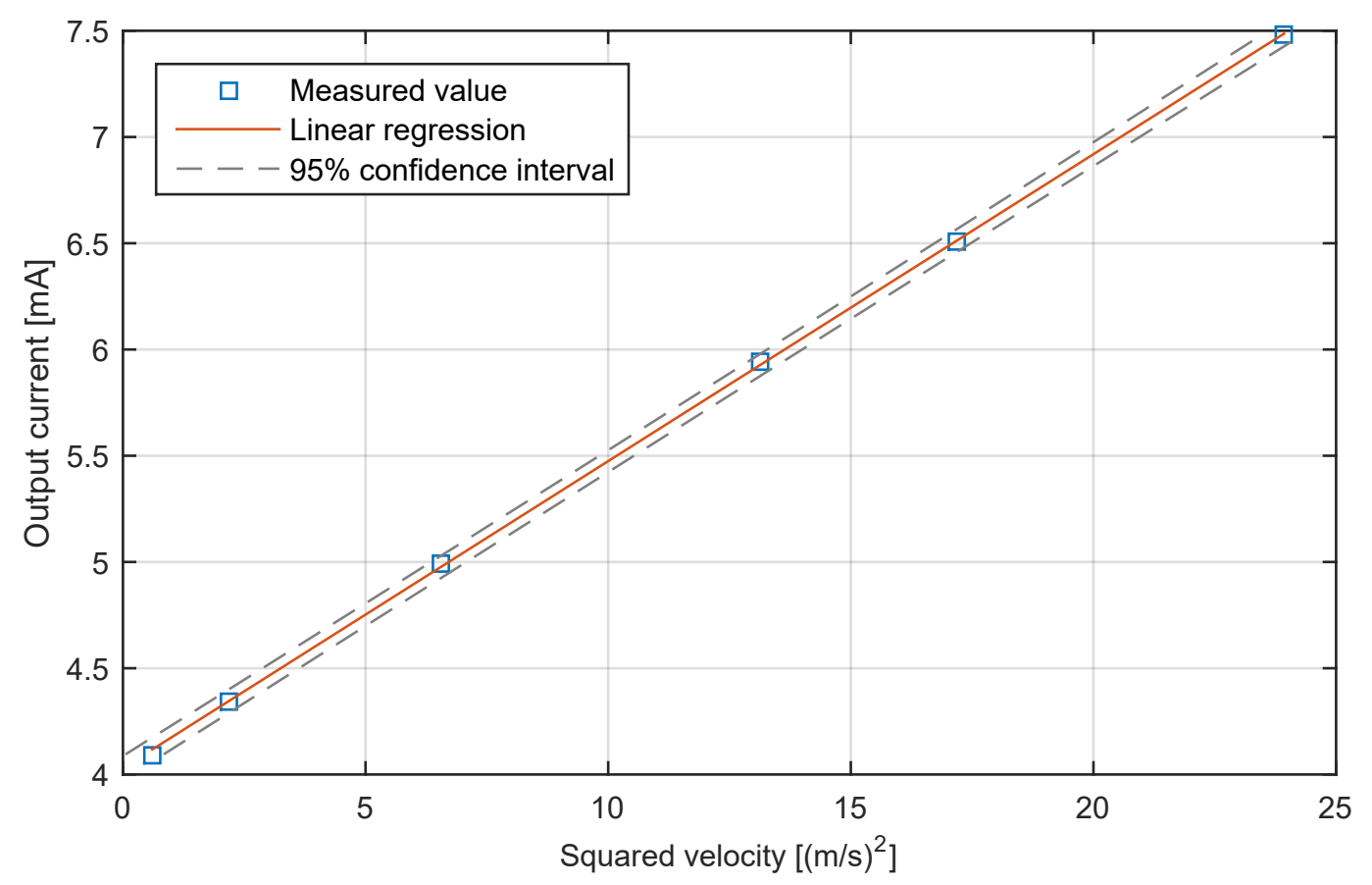

Source: Author

The obtained expression of advance velocity $v_{a}$ is adopted in other expressions, such as to compute the advance ratio $J$. Next, it is demonstrated how to obtain the blockage ratio and its respective propagation of uncertainty.

\section{B.7.4 Blockage ratio verification}

The propagation of uncertainty of $\lambda$ is given by:

$$
u_{\lambda}^{2}=\left|\frac{\partial \lambda}{\partial \alpha}\right|^{2} u_{\alpha}^{2}+\left|\frac{\partial \tau}{\partial \tau}\right|^{2} u_{\tau}^{2}
$$

Since an iterative method for obtaining $\lambda$ is required, expression results for several $\tau$ and $\alpha$ close to the Cavitation Tunnel were interpolated, in order to obtain the partial derivatives.

The propagation of uncertainties of $\alpha$ and $\tau$ are given by:

$$
\begin{gathered}
u_{\alpha}^{2}=\alpha^{2}\left[\left(2 \frac{u_{D_{p}}}{D_{p}}\right)^{2}+\left(\frac{u_{A_{t}}}{A_{t}}\right)^{2}\right] \\
\left(\frac{u_{\tau}}{\tau}\right)^{2}=\underbrace{\left(\frac{u_{T}}{T}\right)^{2}}_{\text {Sec. B.6.1 }}+\underbrace{\left(\frac{u_{\rho}}{\rho}\right)^{2}}_{\text {Sec. B.9 }}+\underbrace{\left(2 \frac{u_{D_{p}}}{D_{p}}\right)^{2}}_{\approx 0}+\underbrace{\left(2 \frac{u_{v^{\prime}}}{v^{\prime}}\right)^{2}}_{\text {Sec. B.7.1 }}
\end{gathered}
$$


Further details about blockage effects are studied and presented in Appendix . C.

\section{B.8 Rotating rate verification}

The rotation is obtained by a count of pulses $\Delta C$ in a given interval $\Delta t$. At each revolution, there are $k_{n}$ pulses. In this way, the expression for the rotation (in units of revolutions per seconds) and its uncertainty are given by:

$$
\begin{gathered}
n=\frac{\Delta C}{k_{n} \Delta t} \\
u_{n}^{2}=n^{2}\left[\left(\frac{u_{\Delta C}}{\Delta C}\right)^{2}+\left(\frac{u_{\Delta t}}{\Delta t}\right)^{2}\right]
\end{gathered}
$$

In this case, the uncertainty is negligible because it is a well-known expression. Also, by performing acquisition through a computer, with very high acquisition frequencies, the measurement uncertainty $u_{\text {disp }}$ is neglected.

\section{B.9 Density and Vapor Pressure}

The water density and vapor pressure are determined by a table presented in ITTC (2011) that correlates these values with the water temperature, determined through interpolation of the form:

$$
\begin{gathered}
\rho=\rho_{0}+\frac{\partial \rho}{\partial \theta} \theta \\
p_{s}=p_{s 0}+\frac{\partial p_{s}}{\partial \theta} \theta
\end{gathered}
$$

The uncertainty of this expression is given by:

$$
\begin{gathered}
u_{\rho}^{2}=\left|\frac{\partial \rho}{\partial \theta}\right|^{2} u_{\theta}^{2}+u_{\text {method, } \rho}^{2} \\
u_{p_{s}}^{2}=\left|\frac{\partial p_{s}}{\partial \theta}\right|^{2} u_{\theta}^{2}+u_{\text {method, }, p_{s}}^{2} \\
u_{\theta}^{2}=u_{d i s p, \theta}^{2}+u_{d i s c r, \theta}^{2}
\end{gathered}
$$

Note that the method has two main approximations: atmospheric and water temperature inside the cavitation tunnel are the same, and the same as in ITTC (2011), 
freshwater. In this way, the uncertainty of method, $u_{\text {method, } \rho}$ and $u_{\text {method,ps }}$, is included, besides the temperature uncertainty.

\section{B.10 Hydrostatic pressure}

Absolute hydrostatic pressure on the axis $p_{h}$ is measured through a pressure transducer, where one of the ports measures hydrostatic pressure while the other, the atmospheric one. With a barometer $p_{a t m}$, the value is converted to absolute pressure. The following expressions summarize the procedure.

$$
\begin{gathered}
p_{h}=p_{r} p_{a t m} \\
u_{p_{h}}^{2}=p_{h}^{2}\left[\left(\frac{u_{p_{r}}}{p_{r}}\right)^{2}+\left(\frac{u_{p_{a t m}}}{p_{a t m}}\right)^{2}\right]+u_{\text {method, } p_{h}}^{2}
\end{gathered}
$$

Once the atmospheric pressure is acquired through an analog reading, a triangular distribution is used for computing the resolution uncertainty.

\section{B.11 Gravity}

According to the data of the Institute of Astronomy, Geophysics and Atmospheric Sciences (IAG) of University of Sao Paulo, using a gravimeter, the measured gravity is $g=9.7864(3) \mathrm{m} / \mathrm{s}^{2}$.

The value and its uncertainty are used for subsequent calculations, as the IAG is located less than $500 \mathrm{~m}$ from the Cavitation Tunnel.

\section{B.12 Cavitation Tunnel Uncertainty}

Aspects related to the cavitation tunnel geometry, such as cross section dimensions and the lever arm used for thrust measurement, are considered without uncertainty.

\section{B.13 Turbulence intensity}

To estimate the turbulence intensity, PIV technique was used in the condition without the propeller. Two cameras were used to increase the image capture zone, i.e., the stereo function was not used. The velocity in the normal component of the image plane is estimated as equal to the vertical component. Three conditions, with different advance velocity, were tested. In each condition, 40 pairs of images were captured, resulting, after 
processing, in the velocity field and its standard deviation. The mean of these 40 velocity fields for each condition are presented in Fig. 101.

Figure 101 - PIV values of velocity

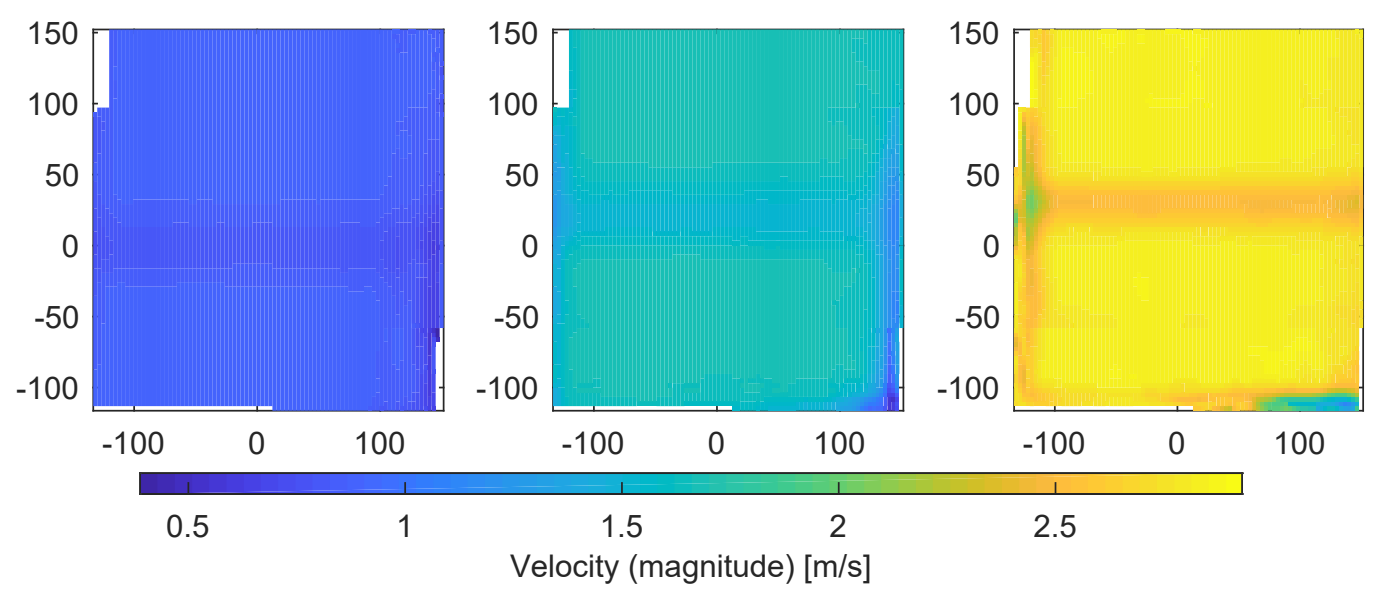

Source: Author

From that, the wake of the axis in the middle of the velocity fields is noticed. Moreover, some regions present inconsistent velocities due to the proximity of the edge of the image. Thus, these regions are not considered for the determination of the turbulence intensity.

As it is a 2D velocity field, the reference velocity is given by Eq. (B.49).

$$
U_{r e f}=\sqrt{\bar{u}^{2}+\bar{v}^{2}+\bar{w}^{2}} \approx \sqrt{\bar{u}^{2}+\bar{w}^{2}+\bar{w}^{2}}
$$

The turbulence kinetic energy $k$, given by Eq. (B.50), is estimated assuming that the root mean square of the velocity fluctuation is equal to the standard deviation of the velocity field obtained by PIV.

$$
k=\frac{1}{2}\left(\overline{u^{\prime 2}}+\overline{v^{\prime 2}}+\overline{w^{\prime 2}}\right) \approx \frac{1}{2}\left(\sigma_{u}^{2}+\sigma_{w}^{2}+\sigma_{w}^{2}\right)
$$

Finally, the turbulence intensity is given by Eq. (B.51).

$$
T_{i}=\frac{\sqrt{\frac{2}{3} k}}{U_{\text {ref }}}
$$

Discounting incoherent values of velocities at edges and due to the axis wake, the average of velocity magnitude, turbulence kinetic energy, and turbulence intensity, is shown for each of the conditions in Tab. 13.

The three results, even varying the advance velocity, have turbulence intensity close to $1 \%$, being the value adopted for all CFD simulations. 
Table 13 - Turbulence intensity

\begin{tabular}{ccc}
\hline mean $U_{\text {ref }}[\mathrm{m} / \mathrm{s}]$ & mean $k\left[(\mathrm{~m} / \mathrm{s})^{2}\right]$ & mean $T_{i}[-]$ \\
\hline 0.8796 & 0.000218 & 0.0123 \\
1.6677 & 0.000620 & 0.0106 \\
2.8285 & 0.001230 & 0.0087 \\
\hline
\end{tabular}





\section{APPENDIX C - Blockage Effects}

\section{C.1 Introduction}

Cavitation Tunnels are commonly used in the naval and ocean engineering fields to investigate the hydrodynamic performance and cavitation pattern of propellers and rudders in conditions with cavitation. One of the main drawbacks of these devices is the occurrence of the so-called blockage effect, which occurs when the flow disturbed by the propeller or rudder is affected by the presence of the tunnel walls, interfering with the measurement of their hydrodynamic force, torque and the cavitation patterns. One way to estimate the blockage influence is to compare the hydrodynamic force and torque measured by a propeller dynamometer in an open-water condition on a Towing Tank with those obtained on a Cavitation Tunnel. However, as the majority of the Towing Tanks do not control the static pressure of the experiments, is not possible to predict the cavitation pattern.

In order to adjust the measurements of a propeller test in a Cavitation Tunnel, this chapter aims to analyze the blockage effect on the IPT's Cavitation Tunnel using CFD tools to obtain a relation between the propeller load, the cavitation area and blockage effect.

The simulations with blockage are carried out with the same methodology seen in Sec. 6, i.e., using a similar mesh topology, but with Realizable $k-\epsilon$ turbulence model. Firstly, steady simulations are conducted, comparing the results with and without the potential correction for the blockage effects. Later, simulations including blockage and cavitation are presented, verifying the influence in the blade cavitation area with the classical potential correlation.

This chapter is based on a previous paper published in Katsuno and Dantas (2017).

\section{C.2 Potential blockage correction}

Glauert (1983) proposes an one-dimensional potential formulation to correct the effect of Wind Tunnel interference when a propeller is tested. The adopted hypotheses were: the fluid is inviscid and incompressible; any fluid rotation inside the streamtube is neglected; the fluid velocity is considered uniform along each cross section of streamtube; and the pressure is assumed constant along each cross section of the streamtube.

Considering the dimensionless coefficients presented in Glauert (1983), $\lambda$ as the ratio between the upstream velocity and the equivalent free velocity, $\tau$ as defined at 
Eq. (C.1) and $\alpha$ as the blockage ratio, defined at Eq. C.2.

$$
\begin{gathered}
\tau=\frac{4 J^{2} K T}{\pi} \\
\alpha=\frac{A_{p}}{A_{t}}=\frac{\pi D_{p}^{2}}{4} \frac{1}{A_{t}}
\end{gathered}
$$

Figure 102 shows the relations between $\tau$ and $\lambda$ for several $\alpha$ used in this section.

Figure 102 - Equivalent free velocity considering blockage effect

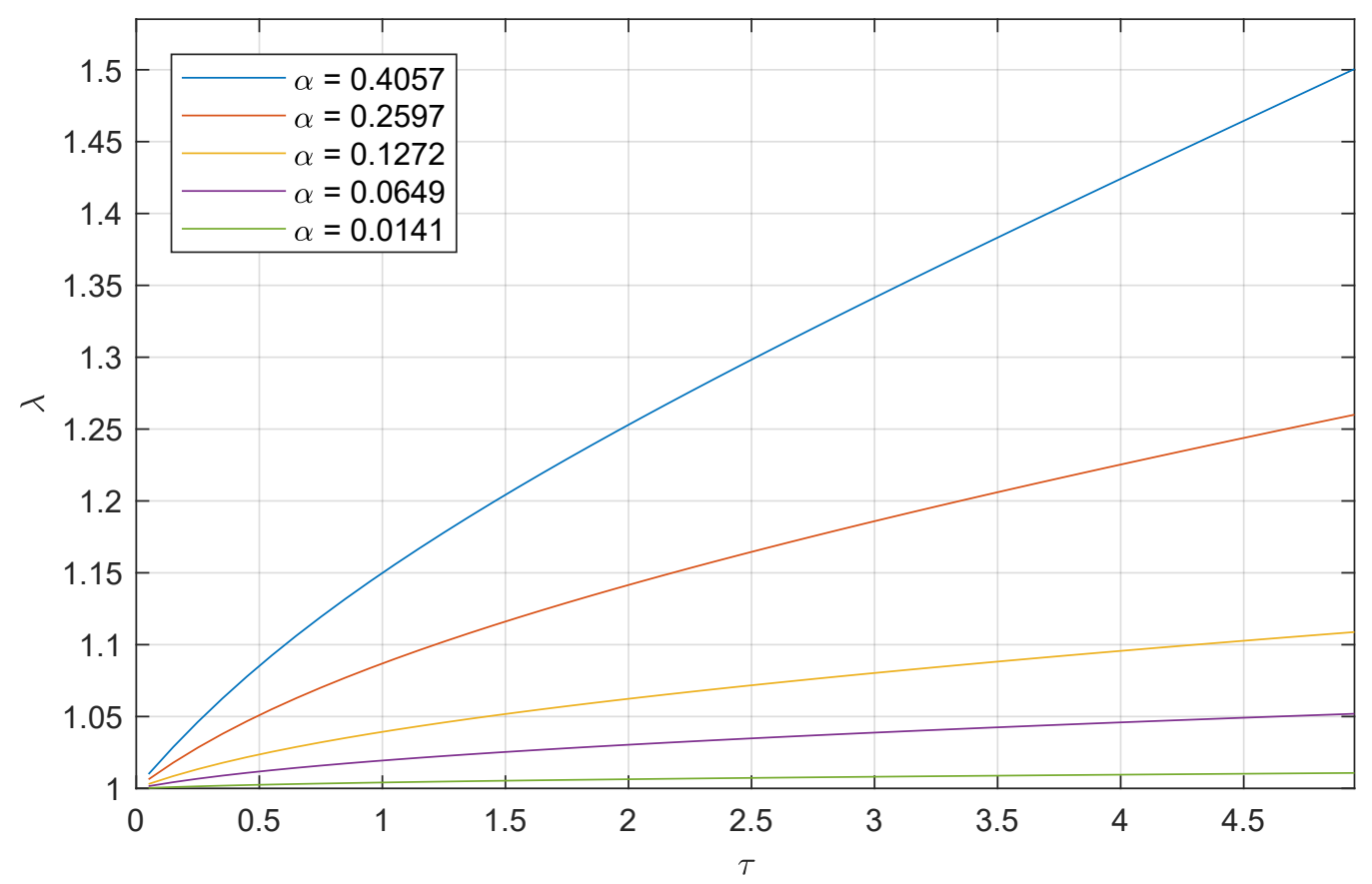

Source: Author

\section{C.3 Domain Geometry and Boundary Conditions}

To simulate blockage, two regions are used: one, with a cylindrical shape, which contains the propeller inside with rotational movement; and another one, which represents the tunnel, with an empty space to contain the propeller region. Five sizes of tunnel cross sections will be simulated and compared. Figure 103 and Tab. 14 show the dimensions of the propeller region, the dimensions of the tunnel and the boundary conditions. The two regions interact through the internal interfaces.

For steady-state simulations that consider blockage, but without cavitation model, MRF was used in the propeller region. A steady-state simulation has a considerably low computational cost in comparison with the unsteady one, but the technique is an 
Figure 103 - Domain geometric dimensions and boundary conditions of simulations with blockage
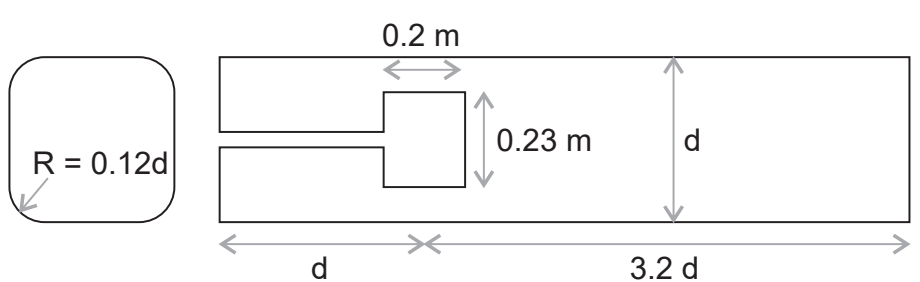

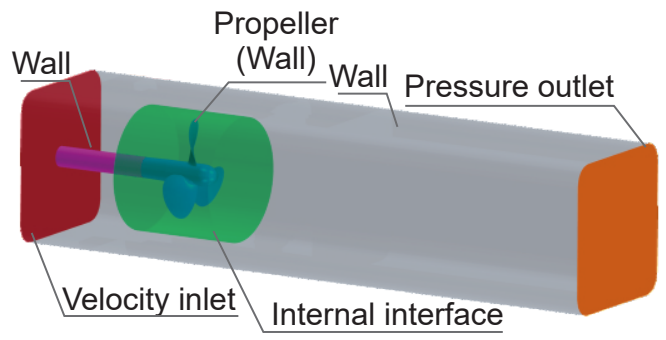

Source: Author

Table 14 - Dimensions of numerical model of cavitation tunnel.

\begin{tabular}{ccc}
\hline$d[\mathrm{~m}]$ & $A_{t}\left[\mathrm{~m}^{2}\right]$ & $\alpha$ \\
\hline 0.28 & 0.077 & 0.4057 \\
0.35 & 0.121 & 0.2597 \\
0.50 & 0.247 & 0.1272 \\
0.70 & 0.484 & 0.0649 \\
1.50 & 2.222 & 0.0141 \\
\hline
\end{tabular}

approximation to represent the propeller rotation, as the propeller do not rotate in the simulation, only the movement is imposed.

For simulations with cavitation model, an implicit unsteady model was adopted. In this case, the Rigid Body Motion (RBM) is used to represent the propeller rotation. Unlike the MRF model, RBM rotates the propeller region. In each time step, the internal interface between propeller and tunnel regions needs to be reassigned. That way, RBM is only possible with time-dependency simulations.

\section{C.4 Mesh Topology}

Based on the lengths of the chosen mesh for the open-water simulations, presented in Sec. 6 the propeller region mesh is similar, with the same dimensions, regardless of the tunnel size.

Figure 104 shows the meshes for all five tunnels dimensions and the propeller mesh. The axis diameter and the empty space to propeller region are the same for all meshes. 
Figure 104 - Adopted tunnel meshes.
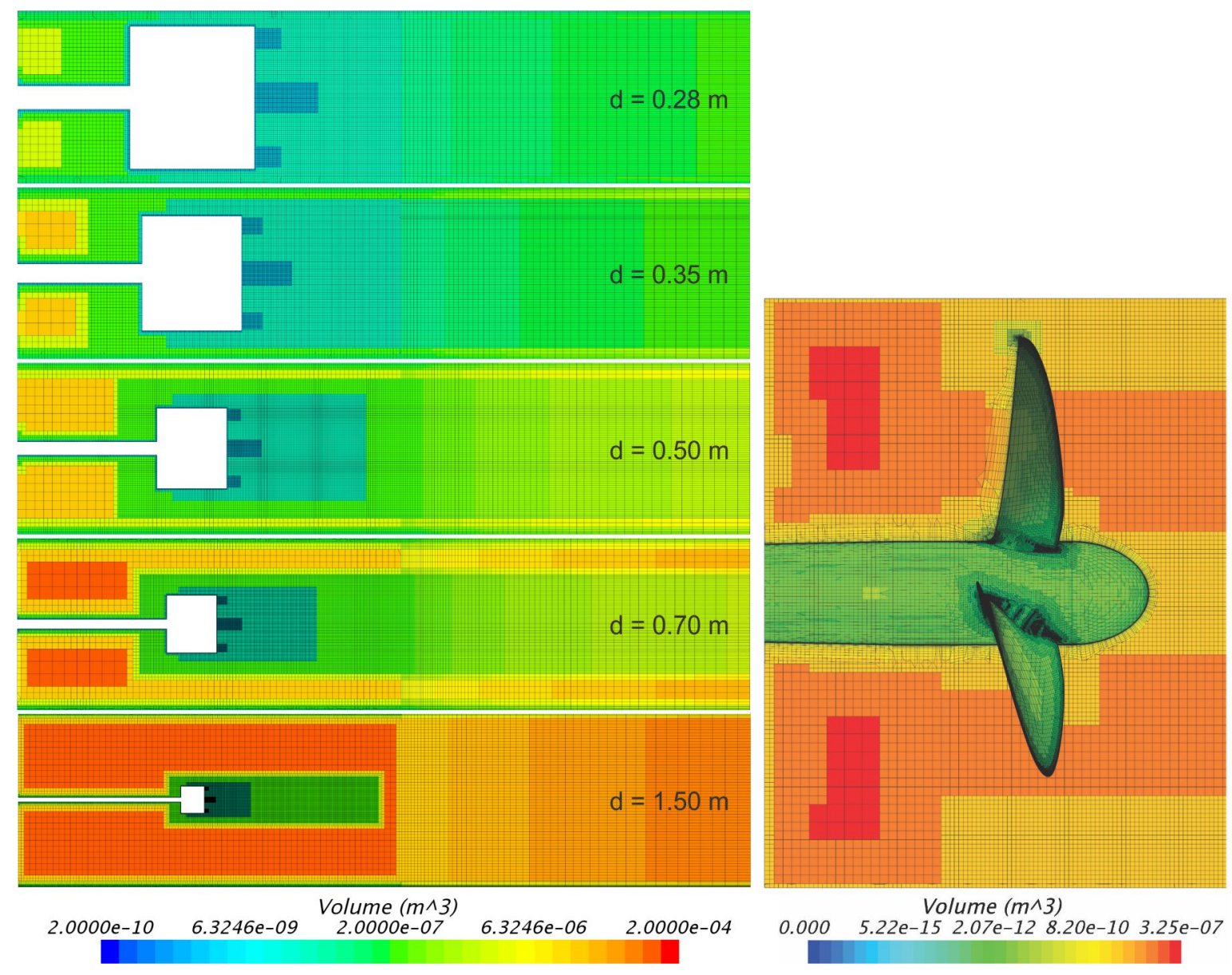

Source: Author

\section{C.5 Steady-state blockage results}

Figure 105 shows the propeller performance coefficients for the presented blockage ratios, compared with experimental values and after applying the blockage correction. The results produced by using the MRF model are close to both experimental results and open-water simulations.

Another form to observe the blockage influence is plotting the propeller coefficients versus the blockage ratio, as it can be seen in Fig. 106. There is a linear tendency of the coefficients in function of $\alpha$, showing their convergences. 
Figure 105 - Propeller performance coefficients without (left) and with blockage correction (right)

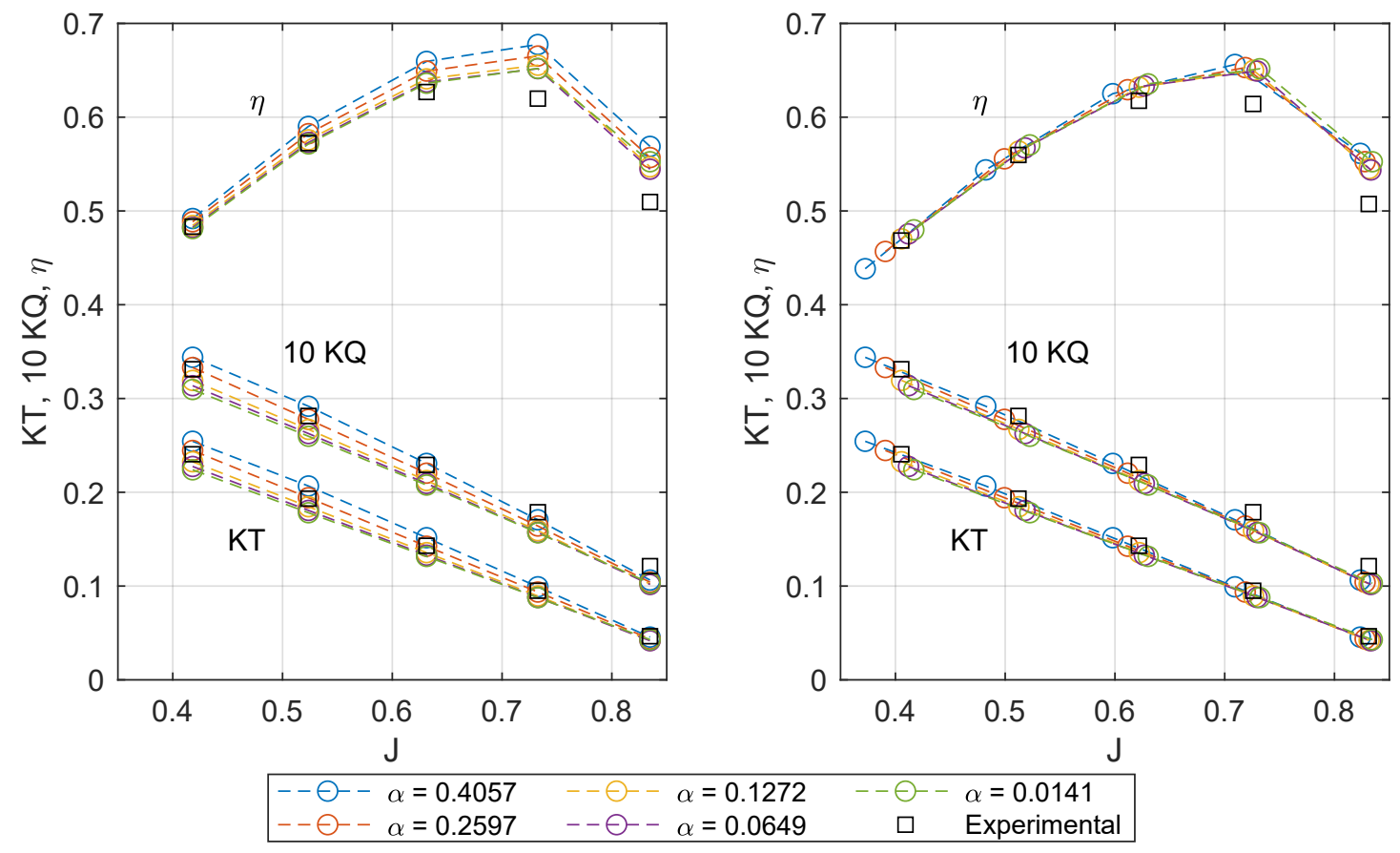

Source: Author

Figure 106 - Propeller performance coefficients versus blockage ratio

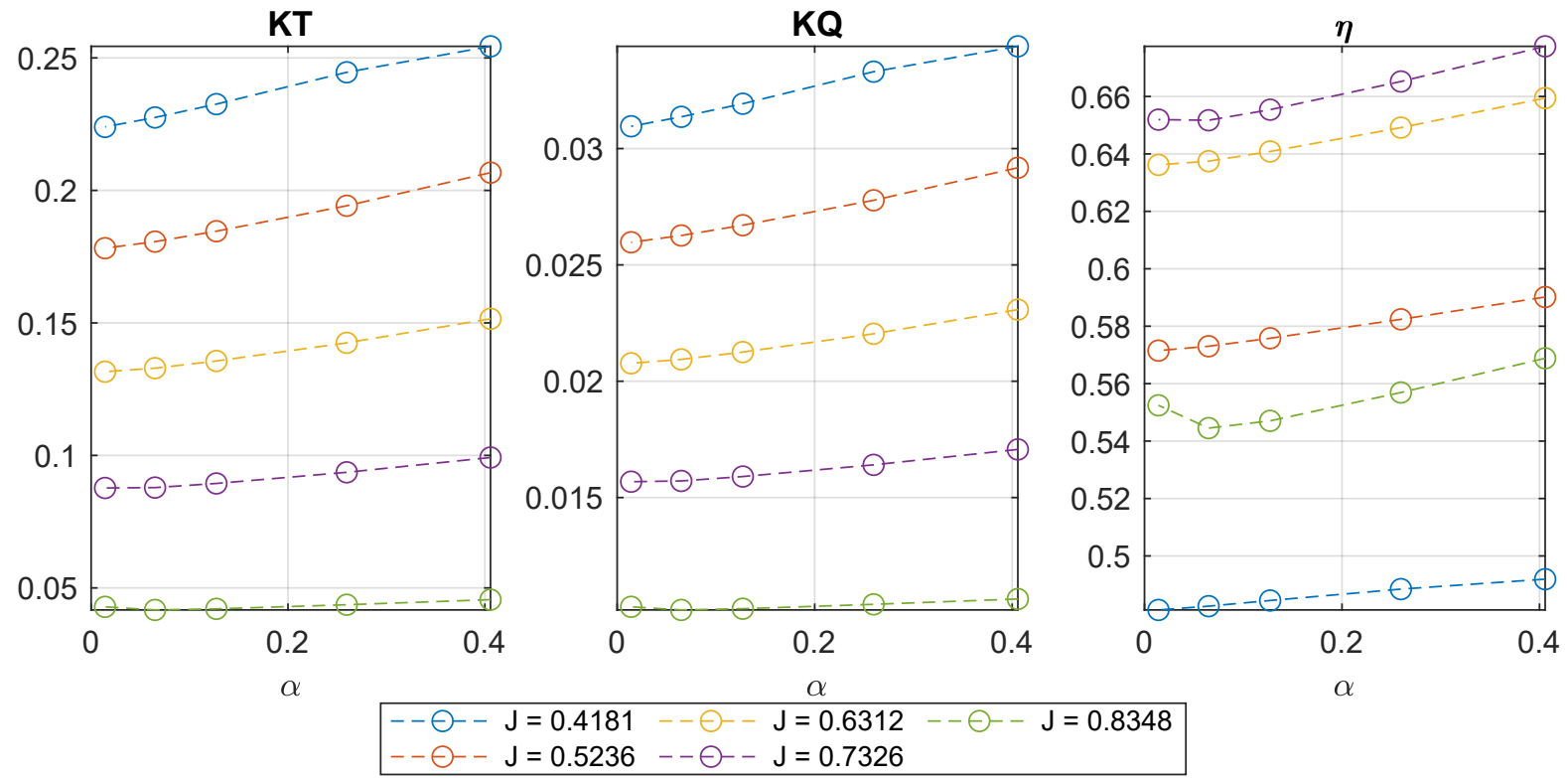

Source: Author 


\section{C.6 Cavitation and blockage results}

Considering the cavitation effects and using RBM to simulate the propeller rotation, this section presents the influence of the blockage effect in the propeller force, torque and in the cavitation area. Cavitation number of $\sigma=3$ is adopted. The propeller coefficients are shown in Fig. 107 in function of $J$, compared with experimental values and after applying the blockage correction.

Figure 107 - Propeller performance coefficients without (left) and with blockage correction (right)

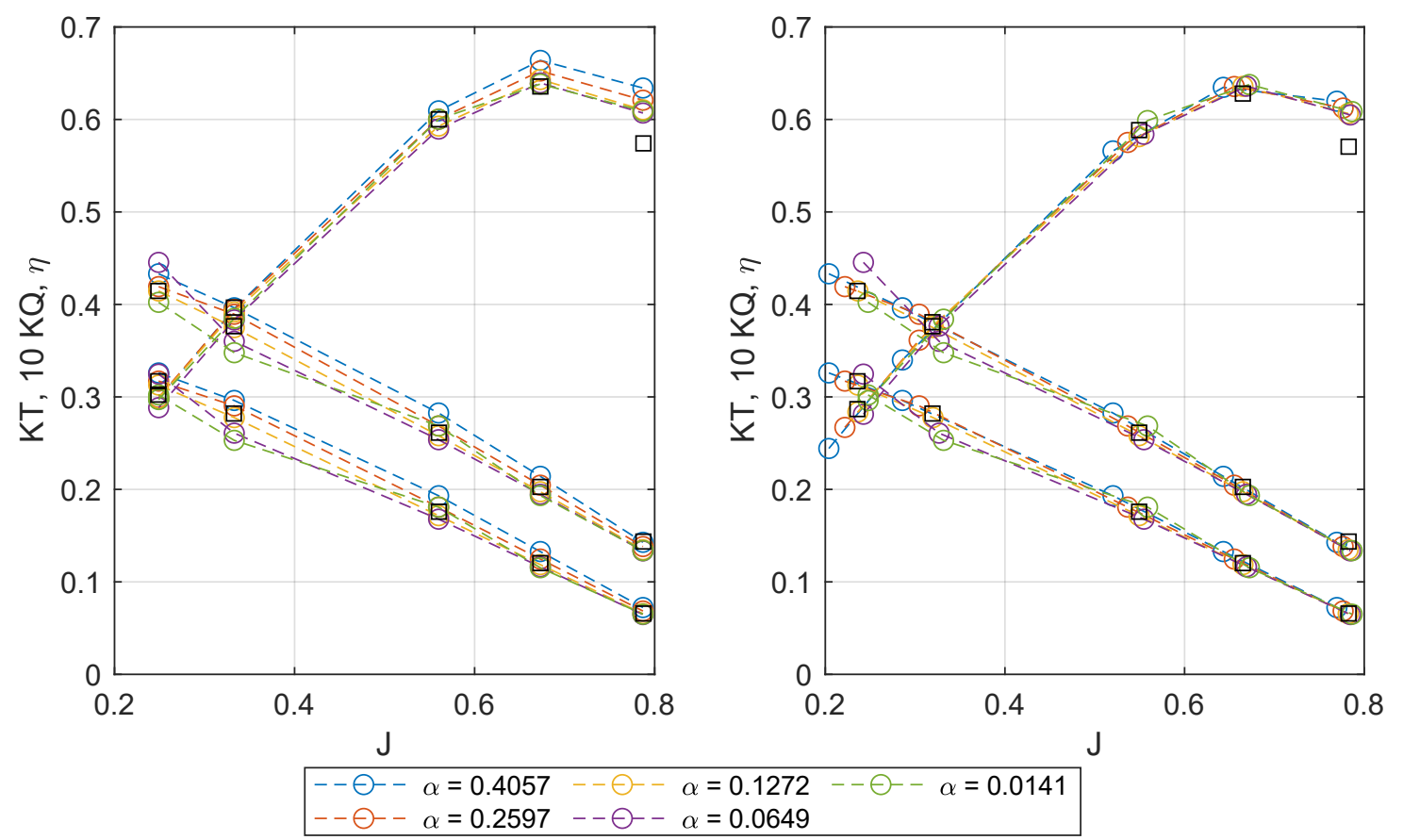

Source: Author

Plotting the propeller coefficients versus the blockage ratio is shown in Fig. 108 in which one can observe there is a linear tendency of the coefficients in function of $\alpha$.

The influence of blockage on cavitation can be observed in Fig. 109, in which $p_{c a v}$ is defined as the percentage of the suction face (back side) covered by cavitation. The numerical treatment to obtain this percentage is the superficial average of suction face with the volume fraction of vapor. Also it is shown in the figure the comparative of cavitation pattern between these two extreme values through an iso-surface 0.5 of volume fraction of vapor, in which the area covered by cavitation from the lower to higher value of blockage ratio increased about $55 \%$. 
Figure 108 - Propeller performance coefficients without (left) and with blockage correction (right)

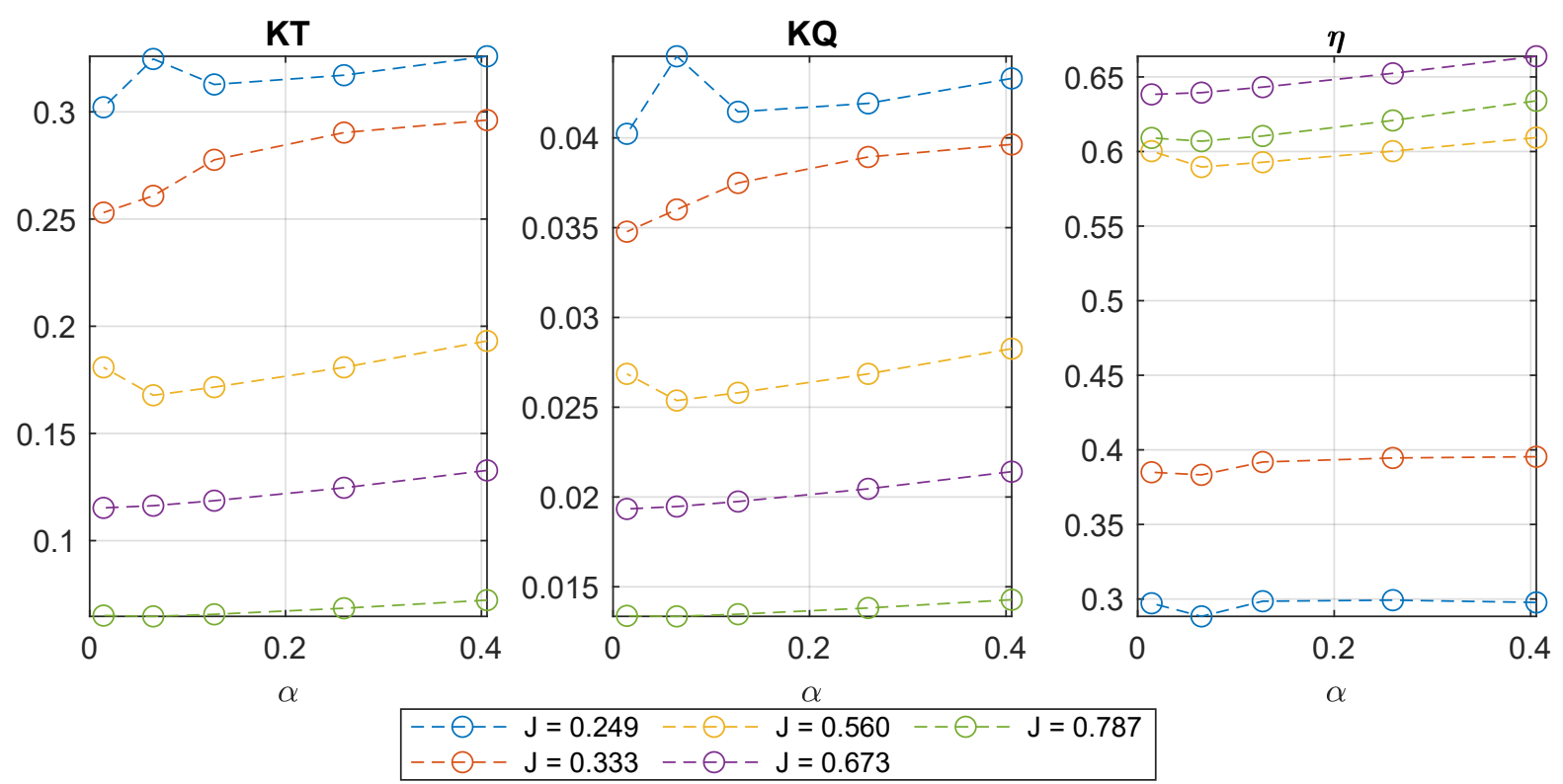

Source: Author

Figure 109 - Influence of blockage ratio in cavitation (left); and comparative between two $\alpha$ for the same $J=0.249$. Suction face. Iso-surface 0.5 of volume fraction of vapor (right)
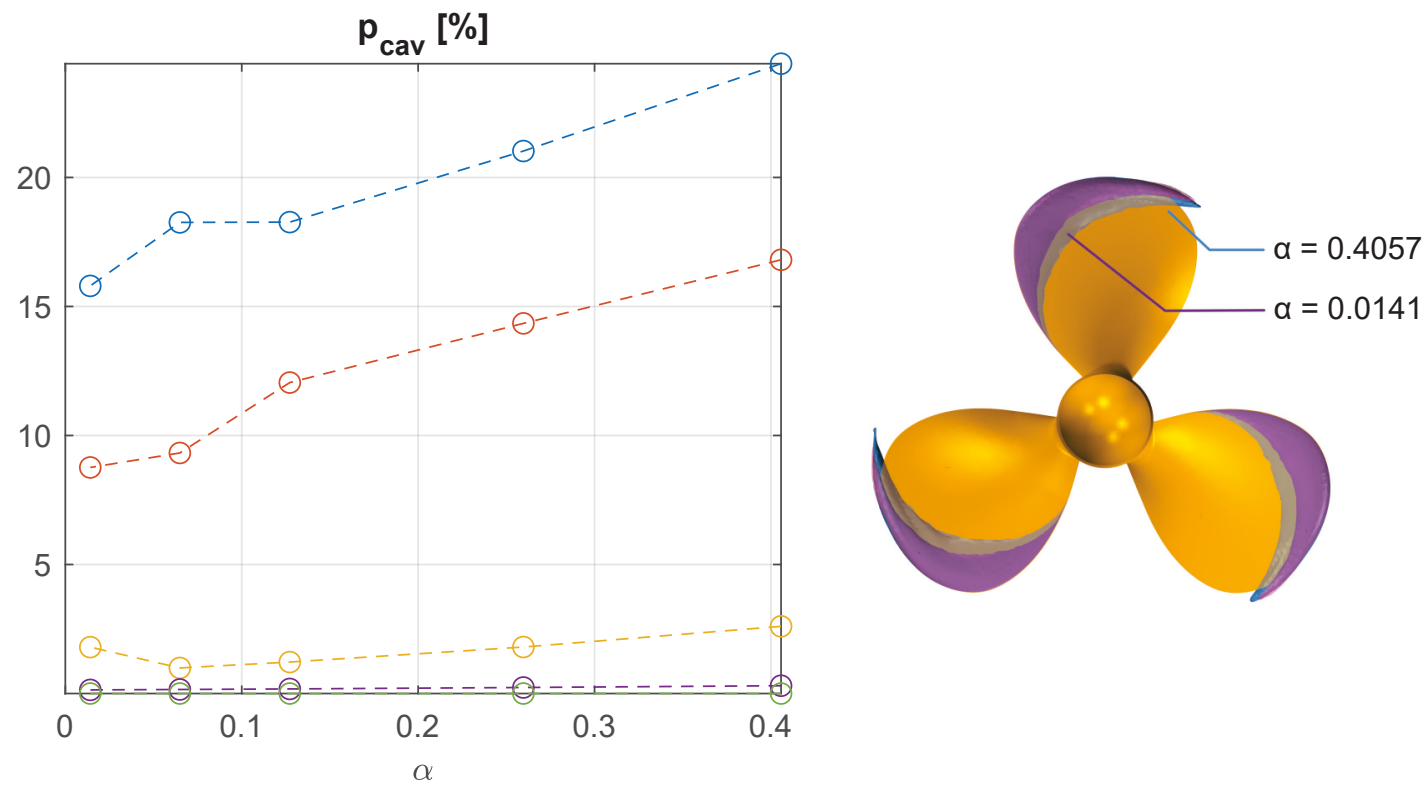

Source: Author 


\section{C.7 Discussions}

The results show good accuracy in simulations compared with experimental data. As it can be noticed, the percentage of propeller covered by cavitation is considerably affected by the blockage effects.

The Glauert model corrected the dependence of blockage ratio in the propeller hydrodynamic force and torque, being a reasonable choice for similar propeller and tested conditions. However, it was noted a high influence of blockage ration in the cavitation area, in which is not contemplated in Glauert model. 